\title{
Determination of Alternative Fuels Combustion Products: Phase I Report
}

\author{
Kevin A. Whitney \\ Southwest Research Institute
}

NREL Technical Monitors: Brent Bailey and Chris Colucci

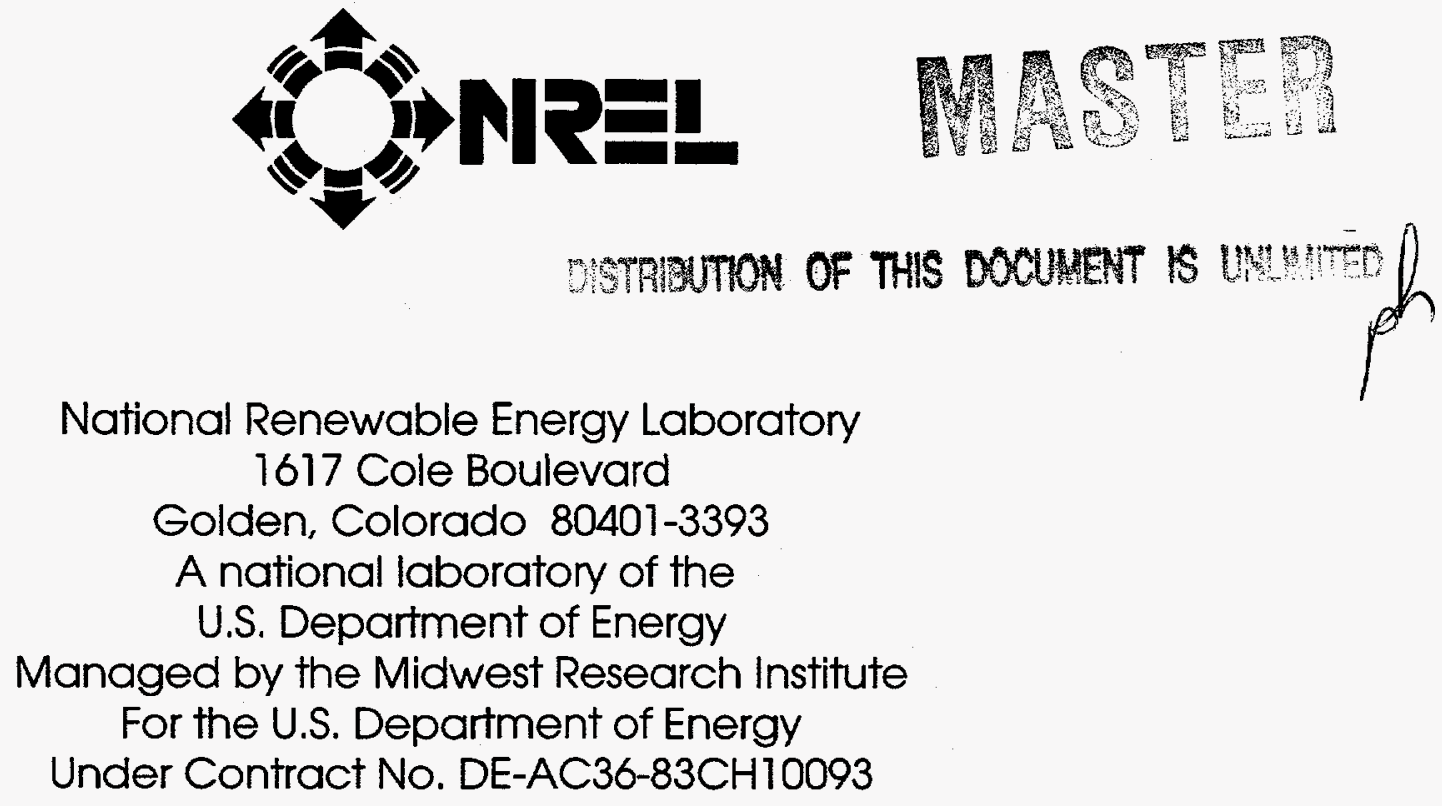

Prepared under Subcontract Number YAW-3-13253-01

September 1997 


\section{FOREWORD}

This project was conducted for the National Renewable Energy Laboratory (NREL) by the Department of Emissions Research of Southwest Research Institute (SwRI). This program was authorized by NREL Subcontract No. YAW-3-13253-01. Operation and management of NREL is conducted by Midwest Research Institute for the United States Department of Energy under Prime Contract No. DE-AC3683CH10093. This program was initiated on October 1, 1993, and testing was completed in October, 1994. This project was based on SwRI Proposal 08-14326 to NREL and was identified within SwRI as Project 086068. The NREL Technical Monitors for this program were Mr. Brent Bailey and Mr. Chris Colucci of NREL Alternative Fuels Division, Golden, Colorado. The SwRI Project Manager was Dr. Lawrence R. Smith, and the Project Leader was Mr. Kevin A. Whitney. Mr. Jimmie Chessher, Laboratory Supervisor, was responsible for emission testing.

ABSTRACT

This report describes the laboratory effort to identify and quantify organic exhaust species generated from alternative-fueled light-duty vehicles operating over the Federal Test Procedure on compressed natural gas, liquefied petroleum gas, methanol, ethanol, and reformulated gasoline. The exhaust species from these vehicles were identified and quantified for fuel/air equivalence ratios of $0.8,1.0$, and 1.2, nominally, and were analyzed with and without a vehicle catalyst in place to determine the influence of a catalytic converter on species formation. 


\section{DISCLAMMER}

Portions of this document may be illegible in electronic image products. Images are produced from the best available original document. 
LIST OF FIGURES $\ldots \ldots \ldots \ldots \ldots \ldots \ldots \ldots \ldots \ldots \ldots \ldots \ldots \ldots \ldots \ldots \ldots \ldots \ldots, \mathrm{v}$

LIST OF TABLES $\ldots \ldots \ldots \ldots \ldots \ldots \ldots \ldots \ldots \ldots \ldots \ldots \ldots \ldots \ldots \ldots \ldots \ldots \ldots \ldots \ldots \ldots$

ACRONYMS AND ABBREVIATIONS $\ldots \ldots \ldots \ldots \ldots \ldots \ldots \ldots \ldots \ldots \ldots \ldots \ldots \ldots \ldots \ldots \ldots \ldots$

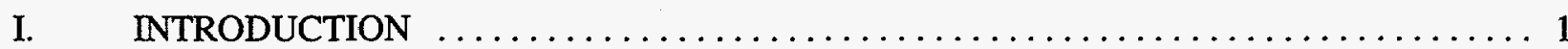

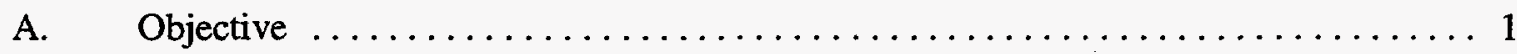

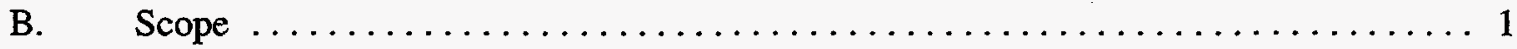

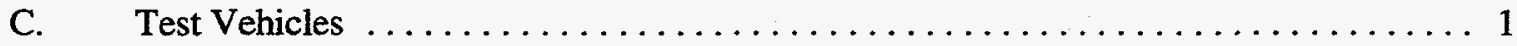

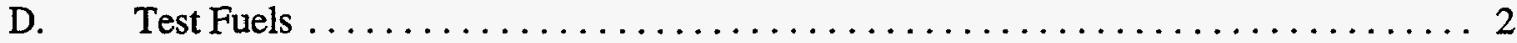

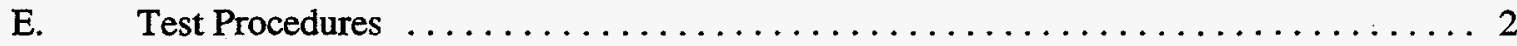

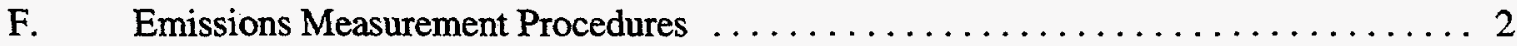

II. GENERAL EQUIPMENT, INSTRUMENTS, PREPARATIONS,

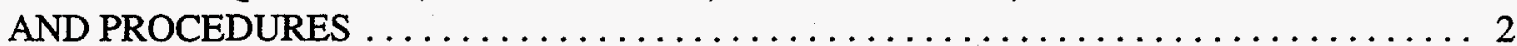

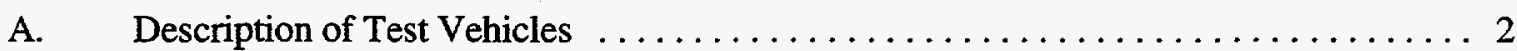

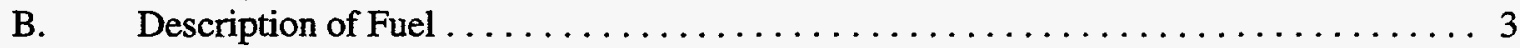

C. Dynamometer and Constant Volume Sampling System $\ldots \ldots \ldots \ldots \ldots \ldots \ldots \ldots$

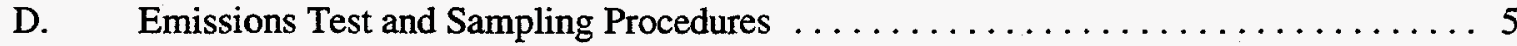

E. Exhaust Emissions Analyses Procedures $\ldots \ldots \ldots \ldots \ldots \ldots \ldots \ldots \ldots \ldots \ldots$

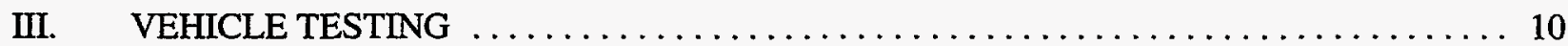

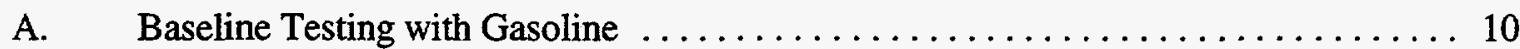

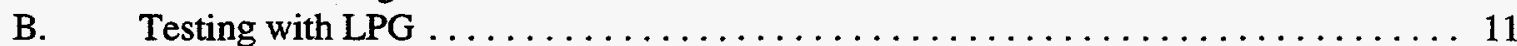

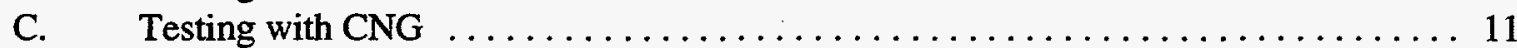

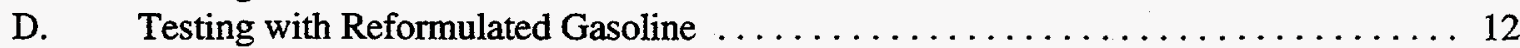

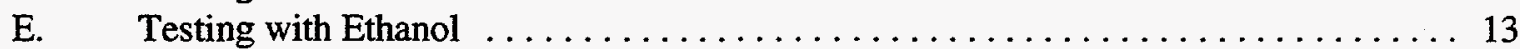

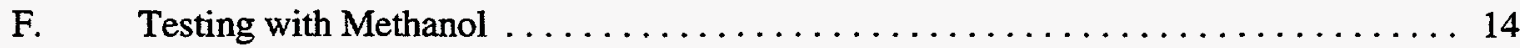

G. Fuel/Air Equivalence Ratios Achieved During Testing $\ldots \ldots \ldots \ldots \ldots \ldots \ldots \ldots$

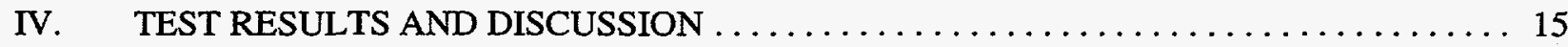

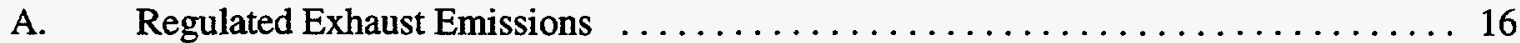

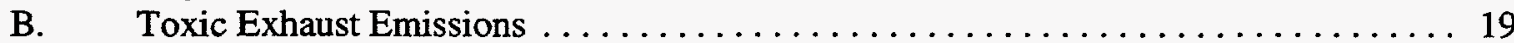

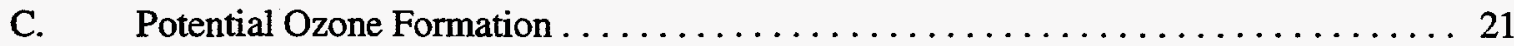


TABLE OF CONTENTS (Cont'd).

Page

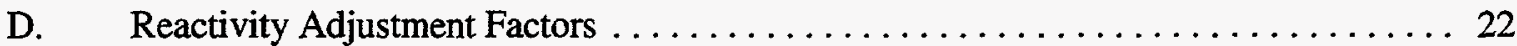

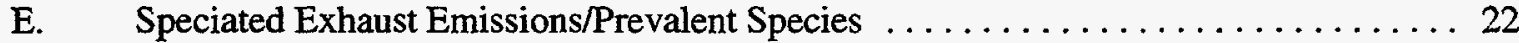

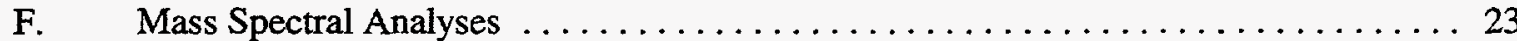

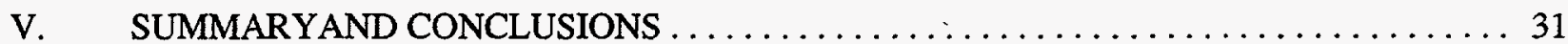

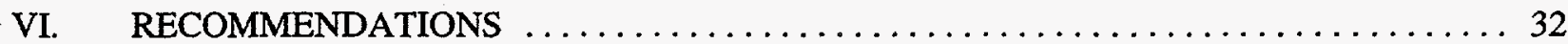

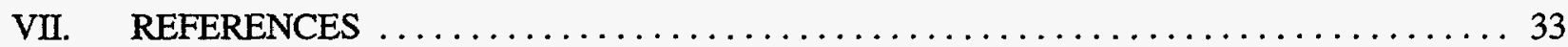

\section{APPENDICES}

A. COMPUTER PRINTOUT OF EMISSIONS DATA FROM "CHECK-OUT" FTP OF CHEVROLET LUMINA

B. COMPUTER PRINTOUTS OF EMISSIONS DATA FROM REFORMULATED GASOLINE BASELINE FTPS OF CHEVROLET LUMINA

C. COMPUTER PRINTOUTS OF EMISSIONS DATA FROM FTPS WITH LPG

D. COMPUTER PRINTOUTS OF EMISSIONS DATA FROM FTPS WITH CNG

E. COMPUTER PRINTOUTS OF EMISSIONS DATA WITH REFORMULATED GASOLINE

F. COMPUTER PRINTOUTS OF EMISSIONS DATA WITH ETHANOL

G. COMPUTER PRINTOUTS OF EMISSIONS DATA WITH METHANOL

H. MAXIMUM INCREMENTAL REACTIVITY ADJUSTMENT FACTORS (MMRs)

I. AVERAGE SPECIATED EMISSIONS RESULTS FROM FTPS WITH LPG

J. AVERAGE SPECIATED EMISSIONS RESULTS FROM FTPS WITH CNG

K. AVERAGE SPECLATED EMISSIONS RESULTS FROM FTPS WITH REFORMULATED GASOLINE

L. AVERAGE SPECIATED EMISSIONS RESULTS FROM FTPS WITH ETHANOL

M. AVERAGE SPECIATED EMISSIONS RESULTS FROM FTPS WITH METHANOL 


\section{LIST OF FIGURES}

Figure

Page

1 FTP Driving Schedule Showing Test Segments $\ldots \ldots \ldots \ldots \ldots \ldots \ldots \ldots \ldots \ldots$

2 Schematic of Mass Spectral Analyses Sampling System $\ldots \ldots \ldots \ldots \ldots \ldots \ldots$

\section{LIST OF TABLES}

Table

Page

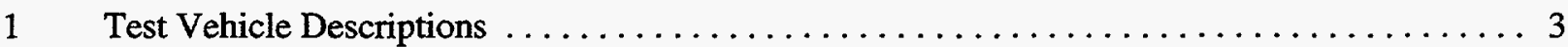

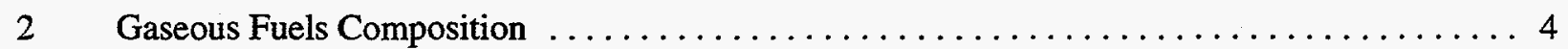

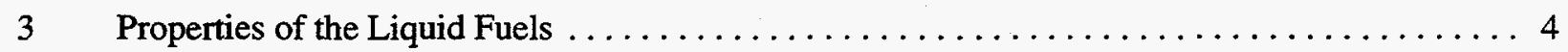

4 Chassis Dynamometer Settings $\ldots \ldots \ldots \ldots \ldots \ldots \ldots \ldots \ldots \ldots \ldots \ldots \ldots \ldots \ldots$

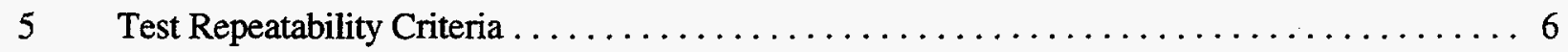

6 Exhaust Species Collection and Analysis Methods $\ldots \ldots \ldots \ldots \ldots \ldots \ldots \ldots \ldots$

7 Initial Lumina Test Results with Gasoline (U.S. FTP Cycle) $\ldots \ldots \ldots \ldots \ldots \ldots \ldots \ldots \ldots$

$8 \quad$ Lumina FTP Results Compared to Baseline $\ldots \ldots \ldots \ldots \ldots \ldots \ldots \ldots \ldots \ldots \ldots \ldots$

9 Initial Corsica Test Results with Ethanol (U.S. FTP Cycle) $\ldots \ldots \ldots \ldots \ldots \ldots \ldots \ldots$

10 FTP Results Before and After Fuel Pump Replacement $\ldots \ldots \ldots \ldots \ldots \ldots \ldots \ldots \ldots \ldots$

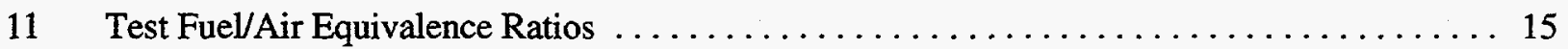

12 Summary of Average FTP Emissions Without Catalyst $\ldots \ldots \ldots \ldots \ldots \ldots \ldots \ldots \ldots 17$

13 Summary of Average FTP Emissions With Catalyst $\ldots \ldots \ldots \ldots \ldots \ldots \ldots \ldots \ldots$

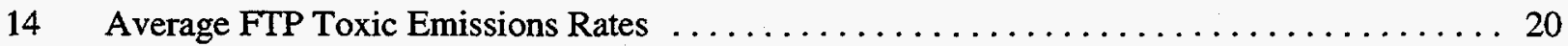

15 Potential Ozone Formation $(\mathrm{mg} / \mathrm{mile}) \ldots \ldots \ldots \ldots \ldots \ldots \ldots \ldots \ldots \ldots \ldots \ldots \ldots \ldots \ldots$

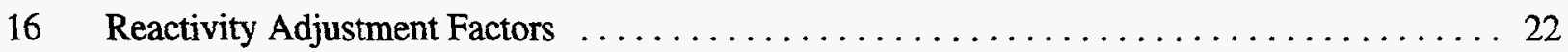

17 Prominent Non-Methane Exhaust Species Identified by 24

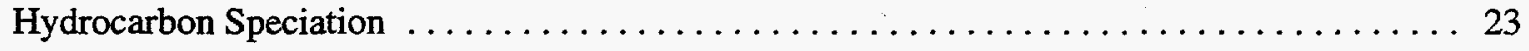

18 Speciated Compounds Conformed by GC-MS Analysis $\ldots \ldots \ldots \ldots \ldots \ldots \ldots \ldots$ 


\section{ACRONYMS AND ABBREVIATIONS}

$\begin{array}{ll}\text { CARB } & \text { California Air Resources Board } \\ \text { CDM } & \text { Calibration/Display Module } \\ \text { cfm } & \text { cubic foot per minute } \\ \text { CFR } & \text { Code of Federal Regulations } \\ \text { CNG } & \text { Compressed Natural Gas } \\ \text { CO } & \text { Carbon Monoxide } \\ \text { CO } & \text { Carbon Dioxide } \\ \text { CVS } & \text { Constant Volume Sampler } \\ \text { DNPH } & \text { 2,4-dinitrophenylhydrazine } \\ \text { E85 } & \text { 85\% denatured ethanol and 15\% gasoline } \\ \text { E100 } & \text { 100\% ethanol } \\ \text { ECM } & \text { Engine Control Module } \\ \text { EGO } & \text { Exhaust Gas Oxygen } \\ \text { EPA } & \text { Environmental Protection Agency } \\ \text { EtOH } & \text { Ethanol } \\ \text { FID } & \text { Flame Ionization Detector } \\ \text { FTP } & \text { Federal Test Procedure } \\ \text { GC } & \text { Gas Chromatograph } \\ \text { GC/FID } & \text { Gas Chromatograph/Flame Ionization Detector } \\ \text { GC/MS } & \text { Gas Chromatograph/Mass Spectrometry } \\ \text { hp } & \text { horsepower } \\ \text { HC } & \text { Hydrocarbons } \\ \text { IAC } & \text { Idle Air Control } \\ \text { lb } & \text { pound } \\ \text { L/min } & \text { liters per minute } \\ \text { LPG } & \text { Liquefied Petroleum Gas } \\ \text { M85 } & \text { 85\% methanol and 15\% gasoline } \\ \text { MEOH } & \text { methanol } \\ \text { mg } & \text { milligram } \\ \text { MIR } & \text { Maximum Incremental Reactivity } \\ \text { mph } & \text { miles per hour } \\ \text { MS } & \text { Mass Spectrometer } \\ \text { MTBE } & \text { methyl tertiary-butyl ether } \\ \text { NDIR } & \text { nondispersive infrared } \\ \text { NMOG } & \text { Non-Methane Organic Gas } \\ \text { NO } & \text { Oxides of Nitrogen } \\ \text { OD } & \text { Overdrive } \\ \text { OEM } & \text { Original Equipment Manufacturer } \\ \text { ppb } & \text { parts per billion } \\ \text { RAF } & \text { Reactivity Adjustment Factor } \\ \text { RFG } & \text { Reformulated Gasoline } \\ \text { RVP } & \text { Reid Vapor Pressure } \\ \text { SAE } & \text { Society of Automotive Engineers } \\ \text { scfm } & \text { standard cubic feet per minute } \\ \text { THC } & \text { Southwest Research Institute } \\ & \text { Total Hydrocarbons } \\ & \end{array}$


Total Hydrocarbons as determined by a Flame Ionization Detector

$\mathrm{THC} / \mathrm{GC}$

Total Hydrocarbons as determined by a Gas Chromatograph

TLEV

Transitional Low Emission Vehicle

UDDS

Urban Dynamometer Driving Schedule

UEGO

uv

Universal Exhaust Gas Oxygen

ultraviolet

VIN

Vehicle Identification Number 


\section{INTRODUCTION}

In recent years, both government and industry have increased their efforts to develop and advance technology that allows an effective use of alternative transportation fuels, including LPG, CNG, ethanol, and methanol. However, concerns continue over the potential impact of these fuels on air pollution, and on the actual air quality benefits of alternative fuels. For alternative fuels to be viable candidates to replace conventional fuels, it must be demonstrated that their impact on air quality will be no worse than that of existing fuels, and preferably should show characteristics that will improve air quality. To make this determination, it is necessary to identify the engine exhaust species that may be generated from alternative fuels and compare them to those from conventional fuels.

\section{A. Objective}

The objective of this program was to identify volatile organic exhaust species generated from alternative-fueled light-duty vehicles operating over the FTP on CNG, LPG, methanol, ethanol, and RFG. The exhaust species from these vehicles were identified and quantified for fuel/air equivalence ratios of 0.8 , 1.0 , and 1.2, nominally, and were analyzed with and without a catalyst in place to determine the influence of a catalytic converter on species formation.

\section{B. Scope}

A total of five fuels were evaluated under this program (LPG, CNG, methanol, ethanol, and RFG). Each fuel was evaluated on one of two vehicles at nominal fuel/air equivalence ratios of 0.8, 1.0, and 1.2. Duplicate FTP tests were conducted at each equivalence ratio, with and without the catalyst in place, for a total of 12 FTPs per fuel ( 3 equivalence ratios $\times 2$ sampling configurations [with and without catalyst] $\times$ duplicate tests) - a total of 60 FTP tests for the 5 fuels.

THC, $\mathrm{CO}, \mathrm{NO}_{\mathrm{x}}$, and $\mathrm{CO}_{2}$ emissions were quantified, using proportional exhaust gas samples collected in Tedlar bags, in a manner consistent with EPA protocols for light-duty emissions testing. ${ }^{(1)}$ Analytical procedures for conducting hydrocarbon speciation $\left(C_{1}\right.$ to $C_{12}$ hydrocarbons, aldehydes and ketones) were similar to the CRC Auto/Oil Phase II methods. Using the results of speciation, a limited comparison of the ozone-forming potential of each vehicle/fuel combination was undertaken based on the MIR scale as used by CARB for individual exhaust species. Mass spectral analyses were also conducted on exhaust samples to determine exhaust hydrocarbons not identified by other speciation methods.

\section{Test Vehicles}

Two test vehicles were used in this program. A 1993 Chevrolet Lumina equipped with a 3.1-liter V-6 engine was used for the evaluation of LPG, CNG, and RFG combustion products. This vehicle was obtained from a local leasing company and was equipped with appropriate aftermarket conversion kits for operation on CNG and LPG. A 1988 Chevrolet Corsica, obtained from the University of Tennessee, was employed for testing with ethanol and methanol. This vehicle was originally provided to the University by General Motors for participation in the SAE Methanol Marathon. This vehicle was equipped with a CDM which allowed for modification of fuel control system calibrations. Separate calibrations were provided by the University of Tennessee for operation on ethanol and methanol. A more detailed description of the test vehicles is given in Section II.A. 


\section{Test Fuels}

This program used five different test fuels in the FTP evaluations: commercially available LPG, CNG blended by SwRI to reflect average gas composition available in the United States, ${ }^{(2)}$ a fuel blended by Phillips Petroleum to represent California Phase 2 RFG, chemical grade ethanol ( $>99 \%$ pure), and chemical grade methanol (>99\%pure). A more detailed description of the test fuels is given in Section II.B.

\section{E. Test Procedures}

Exhaust emissions were evaluated using the chassis dynamometer portion of the light-duty Federal Test Procedure as described in the CFR, Title 40, Part 86, Subpart B. A more detailed description of the test procedures is given in Section II.C.

\section{F. Emissions Measurement Procedures}

Analyses of exhaust samples included determination of regulated exhaust emissions by CFR methods, hydrocarbon speciation and analyses of aldehyde and ketone according to Auto/Oil Phase II methods, and the determination of trace exhaust species by mass spectral analysis methods. A more detailed description of the emissions measurement procedures is given in Section II.D.

\section{GENERAL EQUIPMENT, INSTRUMENTS, PREPARATIONS, AND PROCEDURES}

This section describes the test vehicles, test fuels, testing and analytical procedures, and general instrumentation used throughout this project.

\section{A. Description of Test Vehicles}

A 1993 Chevrolet Lumina was used to evaluate the LPG, CNG, and RFG fuels while a 1988 Chevrolet Corsica was used to evaluate the ethanol and methanol fuels. Using two different vehicles for these evaluations provided adequate qualitative information concerning exhaust emissions; however, quantitative comparisons of exhaust emissions between the two vehicles has limited value.

The 1993 Chevrolet Lumina used to evaluate LPG, CNG, and RFG was equipped with an appropriate conversion kit for each of the gaseous fuels. Both kits were Mogas ECOLO-Feedback carbureted systems. These kits used feedback from the OEM EGO sensor to regulate a fuel control valve to maintain fuel/air stoichiometry. The kits were installed and tuned by SwRI according to the manufacturer's instructions.

The 1988 Chevrolet Corsica, used to evaluate the ethanol and methanol, originally operated on gasoline and was converted to M85 operation by the University of Tennessee as part of the SAE Methanol Marathon. The vehicle came equipped with a CDM which allows the user to modify the vehicle's fuel injection and ignition timing calibrations. The University has maintained the vehicle as a research tool and leased the vehicle to SwRI for this program. As provided, the vehicle was calibrated to operate on ethanol. A separate calibration for operation on methanol was also provided. Descriptions of both test vehicles are provided in Table 1. 
TABLE 1. TEST VEHICLE DESCRIPTIONS

\begin{tabular}{||l|l|l||}
\hline \multicolumn{1}{|c|}{ Item } & \multicolumn{2}{c||}{ Description } \\
\hline \hline Vehicle & Lumina & Corsica \\
\hline Vehicle Body Style & 2-door Euro sedan & 4-door sedan \\
\hline VIN & 2G1WN14TXP9261375 & 1G1LT51W8JY667124 \\
\hline Vehicle Odometer & 5,685 miles (as received) & 14,705 miles (as received) \\
\hline Engine Family & P1G3.4V8XGZ5/PBO-1K & NA (special waiver) \\
\hline Engine Type & 3.1-liter V-6 & 2.8-liter V-6 \\
\hline Transmissions & 4-speed automatic OD & 5-speed manual \\
\hline Fuel System & multi-point fuel injection & multi-point fuel injection \\
\hline Tires & P205/70R15 & 195/70-R14 \\
\hline
\end{tabular}

To force the vehicles to operate at fuel/air equivalence ratios other than stoichiometry, each vehicle's switching EGO sensor was replaced with a UEGO sensor. The UEGO sensor was capable of operating throughout the range of fuel/air equivalence ratios required by this program. The UEGO signal was passed through a comparator circuit to generate a switching output similar to that of an EGO sensor. The comparator output was connected to the EGO sensor input of each vehicle's fuel control system. By using a comparator switchpoint that was tunable by the operator, the closed loop system was adjusted to control to equivalence ratios other than stoichiometry.

During tests conducted on LPG, CNG, ethanol, and methanol, each vehicle was fitted with a 3-way catalyst designed for application on alcohol-fueled Luminas. According to engineers at GM, this catalyst was more suited for use with CNG and LPG than the OEM gasoline catalyst on the Lumina. In addition, this catalyst was also installed on the Corsica to replace a damaged catalyst that was provided with the vehicle. The performance of the new catalyst was stabilized, using a 24-hour break-in period on an engine dynamometer, before the catalyst was installed on the first test.vehicle. Although the break-in period was not meant to simulate any specific vehicle driving schedule, the break-in can generally be considered equivalent to 4,000 miles of in-use driving. The OEM gasoline catalyst, as received on the Lumina, was used during all testing with reformulated gasoline.

\section{B. Description of Fuel}

This program used five different test fuels in emissions evaluations: commercially available LPG, CNG blended by SwRI to reflect average gas composition available in the United States, ${ }^{(2)}$ a fuel blended by Phillips Petroleum to be representative of California Phase 2 RFG, ethanol of $>99 \%$ purity, and methanol with $>99 \%$ purity. Compositions of the gaseous fuels are given in Table 2 . Note that the LPG used in this program did not meet the HD-5 specification for propylene content, which is $5 \%$ maximum. However, this fuel was represented by the supplier as a motor grade fuel. With the approval of the NREL Technical Monitor, this fuel was used as a representative sample of commercially available LPG. Selected properties for reformulated gasoline and the alcohol fuels are given in Table 3. 
TABLE 2. GASEOUS FUELS COMPOSITION

\begin{tabular}{|c|c|c|}
\hline \multirow[b]{2}{*}{ Fuel Component } & \multicolumn{2}{|c|}{ Fuel Composition (volume percent) } \\
\hline & $\mathbf{L P G}^{\mathbf{a}}$ & $\mathrm{CNG}^{\mathrm{b}}$ \\
\hline Methane & 0.1 & 92.7 \\
\hline Ethane & 2.0 & 3.4 \\
\hline Propylene & 6.1 & 0.0 \\
\hline Propane & 91.4 & 1.3 \\
\hline Butane and higher & 0.4 & 0.0 \\
\hline Nitrogen & 0.0 & 2.6 \\
\hline
\end{tabular}

TABLE 3. PROPERTIES OF THE LIQUID FUELS

\begin{tabular}{||l|r|r|r||}
\hline \multicolumn{1}{|c|}{$\begin{array}{c}\text { Fuel } \\
\text { Properties }\end{array}$} & Phase 2 RFG & Ethanol & Methanol \\
\hline \hline RVP, psi & 6.9 & 2.6 & 5.0 \\
\hline Sulfur, ppm & 33 & $<0.01$ & $<0.01$ \\
\hline Benzene, vol \% & 0.84 & 0.0 & 0.0 \\
\hline Aromatics & 27.1 & 0.0 & 0.0 \\
\hline Olefins, vol \% & 4.6 & 0.0 & 0.0 \\
\hline $\begin{array}{l}\text { Distillation, }{ }^{\circ} \mathrm{F} \\
50 \%\end{array}$ & 210 & 169 & 146 \\
$90 \%$ & 294 & 169 & 146 \\
\hline Oxygenate, vol \% & $11.2 \%$ MTBE & $100 \%$ Ethanol & $99 \%$ Methanol \\
\hline Carbon, wt \% & 84.1 & 52.3 & 37.5 \\
\hline Hydrogen, wt \% & 13.9 & 13.1 & 12.3 \\
\hline Oxygen, wt\% & 2.0 & 34.6 & 50.2 \\
\hline
\end{tabular}

\section{Dynamometer and Constant Volume Sampling System}

A Clayton, Model ECE-50 passenger car chassis dynamometer with a direct-drive variable inertia flywheel system was used for all testing. The inertia weight simulates equivalent weights of vehicles from $1,000 \mathrm{lb}$ to $8,875 \mathrm{lb}$ in 125 -lb increments. Dynamometer settings used for this test program are given in Table 4. A positive displacement-type constant volume sampling system with a nominal flow capacity of $565 \mathrm{scfm}$ was used to dilute the vehicle's exhaust. A cooling fan of 5,000 cfm capacity was used in front of the test vehicle 
during all emissions test driving cycles. In addition, the hood of each test vehicle was fully open during all driving cycles. The cooling fan was off and the hood was closed during soak periods.

TABLE 4. CHASSIS DYNAMOMETER SETTINGS
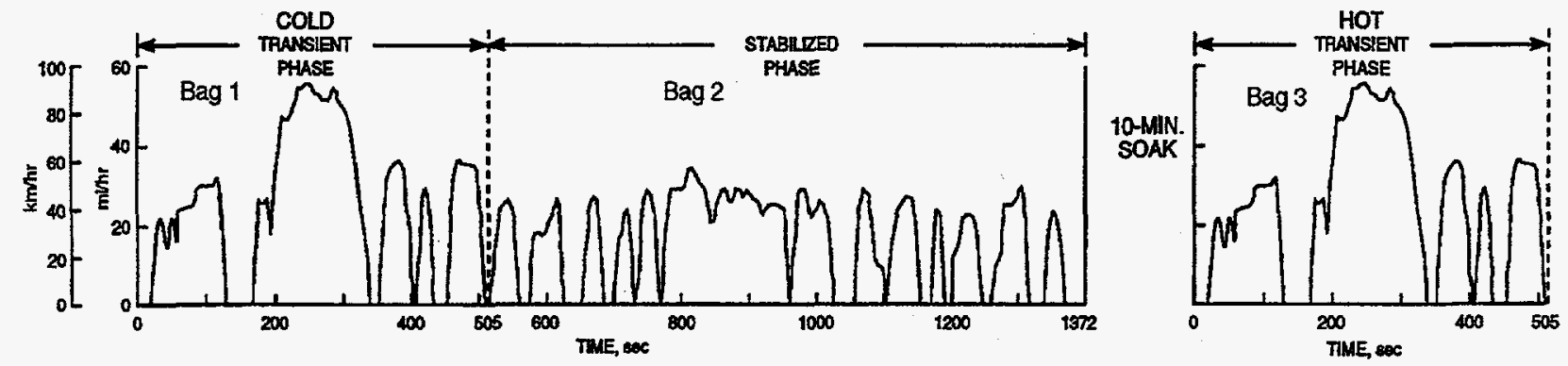

FIGURE 1. FTP DRIVING SCHEDULE SHOWING TEST SEGMENTS

\begin{tabular}{||l|c|c|}
\hline \multicolumn{1}{|c|}{ Item } & Lumina & Corsica \\
\hline \hline Inertial Weight & $4,000 \mathrm{lb}$ & $3,500 \mathrm{lb}$ \\
\hline Actual Road Load @ $50 \mathrm{mph}$ & $6.5 \mathrm{hp}$ & $4.5 \mathrm{hp}$ \\
\hline
\end{tabular}

\section{Emissions Test and Sampling Procedures}

Exhaust emissions were evaluated using the light-duty FTP (1) This procedure uses the UDDS, which is 1,372 seconds in duration. The UDDS is divided into two segments: the first consists of 505 seconds and the second consists of 867 seconds. An FTP is composed of a coldtransient 505 and a cold-stabilized 867 portion, followed by a 10-minute soak, and then a hottransient 505. The FTP driving schedule with its cold- and hot-transient test segments is shown in Figure 1.

A repeat pair of valid FTPs were conducted at each test point, for a total of 12 FTPs per fuel (3 equivalence ratios $\times 2$ sampling configurations [with and without catalyst] $\times$ duplicate tests). Test-to-test repeatability criteria, as developed under the Auto/Oil Air Quality Improvement Research Program, ${ }^{(3)}$ were used for this program and are given in Table 5. Using this method, repeatability ratios were calculated for each repeat pair of valid tests. The repeatability ratio for each exhaust constituent is the ratio of the high and low values obtained from the duplicate tests. If one of the calculated ratios was greater than the criteria given in Table 5, a third FTP test was conducted and averaged with the results of the first two tests. 
TABLE 5. TEST REPEATABILITY CRITERIA

\begin{tabular}{||c|c|}
\hline Exhaust Constituent & Repeatability Ratio \\
\hline \hline $\mathrm{THC}$ & 1.33 \\
\hline $\mathrm{CO}$ & 1.70 \\
\hline $\mathrm{NO}_{x}$ & 1.29 \\
\hline
\end{tabular}

\section{E. Exhaust Emissions Analyses Procedures}

A summary of exhaust emissions sampling and analytical techniques used in this program is given in Table 6. A more detailed description of these techniques is listed below.

TABLE 6. EXHAUST SPECIES COLLECTION AND ANALYSIS METHODS

\begin{tabular}{||l|l|l||}
\hline \multicolumn{1}{|c|}{ Compounds } & \multicolumn{1}{|c|}{ Method of Collection } & \multicolumn{1}{c|}{ Method of Analysis } \\
\hline \hline Total Hydrocarbons & Bag & FID \\
\hline Carbon Monoxide & Bag & NDIR \\
\hline Oxides of Nitrogen & Bag & Chemiluminescent analysis \\
\hline Carbon Dioxide & Bag & NDIR \\
\hline $\begin{array}{l}\text { Hydrocarbon Speciation, } \\
\mathrm{C}_{1}-\mathrm{C}_{12}\end{array}$ & $\mathrm{Bag}$ & GC-FID \\
\hline Aldehydes and Ketones & Impingers containing DNPH & HPLC-UV \\
\hline Methanol and Ethanol & Impingers containing water & GC-FID \\
\hline Unidentified compounds & Sorbant cartridge & Mass spectral analysis \\
\hline
\end{tabular}

\section{Regulated Gaseous Emissions}

THC or OMHCE, $\mathrm{CO}, \mathrm{NO}_{\mathrm{x}}$, and $\mathrm{CO}_{2}$ emissions were quantified in a manner consistent with EPA protocols for light-duty emissions testing as given in the Code of Federal Regulations, Title 40, Part 86, Subpart B. $\mathrm{HC}, \mathrm{CO}, \mathrm{NO}_{x}$, and $\mathrm{CO}_{2}$ were sampled using proportional exhaust gas samples collected in Tedlar bags. HC were measured using an FID. $\mathrm{CO}$ and $\mathrm{CO}_{2}$ were determined using NDIR instruments. $\mathrm{NO}_{\mathrm{x}}$ was measured using a chemiluminescent instrument. Wet absorption techniques were employed to collect methanol, ethanol, and aldehydes for the determination of OMHCE. These techniques are discussed in more detail below.

Methane levels were determined using proportional exhaust gas samples collected in Tedlar bags. A GC equipped with an FID was utilized in accordance with the SAE J1151 procedure to analyze the samples. The GC system was equipped with a packed column 
to resolve methane from other hydrocarbons in the sample. Samples were introduced into a 5$\mathrm{mL}$ sample loop via a diaphragm pump. For analysis, the valve was switched to the inject position and the helium carrier gas swept the sample from the loop toward the detector through a $61 \mathrm{~cm} \times 0.3 \mathrm{~cm}$ Porapak $\mathrm{N}$ column in series with a $122 \mathrm{~cm} \times 0.3 \mathrm{~cm}$ molecular sieve $13 \mathrm{X}$ column. As soon as the methane peak passed into the molecular sieve column, the helium flow was reversed through the Porapak N column to vent. Peak areas were compared to an external calibration standard.

\section{Hydrocarbon Speciation Procedures}

NMOG emissions were determined by hydrocarbon speciation. Analytical procedures for conducting the hydrocarbon speciation $\left(\mathrm{C}_{1}\right.$ to $\mathrm{C}_{12}$ hydrocarbons, aldehydes and ketones, and alcohols) were similar to the CRC Auto/Oil Phase II methods. With these methods, exhaust samples are analyzed for the presence of more than 190 different exhaust species. The sum of the masses of non-methane species is equivalent to the NMOG emissions rate. Three GC procedures and one HPLC procedure were used to identify and quantify specific compounds. A brief description of these procedures is given below.

\section{a. $\quad \mathbf{C}_{1}-\mathbf{C}_{4}$ Species}

The first GC procedure allowed the separation and determination of exhaust concentrations of $\mathrm{C}_{1}-\mathrm{C}_{4}$ individual hydrocarbon species, including methane; ethane; ethylene; acetylene; propane; propylene; propadiene; butane; trans-2-butene; 1-butene; 2methylpropene; 2,2-dimethylpropane; propyne; 1,3-butadiene; 2-methylpropane; 1-butyne; and cis-2-butene. Bag samples were analyzed using a gas chromatograph equipped with an FID. The gas chromatograph system utilized a Hewlett-Packard Model 5890 Series II GC with an FID, two pneumatically operated and electrically controlled valves, and two analytical columns. The carrier gas was helium. An external multiple component standard in zero air was used to quantify the results. Detection limits for the procedure were on the order of $5 \mathrm{ppbC}$ in dilute exhaust for all compounds.

\section{b. $\quad \mathbf{C}_{5}-\mathbf{C}_{12}$ Species}

The second GC procedure provided separation and exhaust concentrations for more than $100 \mathrm{C}_{5}-\mathrm{C}_{12}$ individual $\mathrm{HC}$ compounds. Bag samples were analyzed using a gas chromatograph equipped with an FID. The GC system utilized a Hewlett-Packard Model 5890 Series II GC with an FID, a pneumatically operated and electrically controlled valve, and an analytical column. The carrier gas was helium. An external multiple component standard in zero air was used to quantify the results. Detection limits for the procedure were on the order of $10 \mathrm{ppbC}$ in dilute exhaust for all compounds.

\section{c. Benzene and Toluene}

The third GC procedure used a separate system configured similarly to those mentioned above to determine individual concentrations of benzene and toluene according to the CRC Auto/Oil Phase II Protocol. 


\section{d. Aldehydes and Ketones}

An HPLC procedure was utilized for the analysis of aldehydes and ketones. Samples were collected by bubbling dilute exhaust at a nominal flowrate of $4 \mathrm{~L} / \mathrm{min}$ through chilled glass impingers containing an acetonitrile solution of 2,4-DNPH and perchloric acid. For analysis, a portion of the acetonitrile solution was injected into a liquid chromatograph equipped with a UV detector. External standards of the aldehyde and ketone DNPH derivatives were used to quantify the results. The aldehydes and ketones measured were: formaldehyde, acetaldehyde, acrolein, acetone, propionaldehyde, crotonaldehyde, isobutyraldehyde/methylethylketone (not resolved from each other during normal operating conditions, and so reported together), benzaldehyde, and hexanaldehyde. Detection limits for this procedure were on the order of $0.005 \mathrm{ppm}$ aldehyde or ketone in dilute exhaust.

\section{Alcohols}

The collection of methanol and ethanol in exhaust was accomplished by bubbling exhaust through glass impingers. Each impinger contained $25 \mathrm{~mL}$ of deionized water maintained at ice-bath temperature. Exhaust samples were collected continuously during test cycles at a nominal flow rate of $4 \mathrm{~L} / \mathrm{min}$ through a Teflon sample line held at $102^{\circ} \mathrm{C}\left(215^{\circ} \mathrm{F}\right)$. For analysis, a 1-pL portion of the sample was injected into the GC equipped with an FID and an analytical column. The analytical column was a $0.53-\mathrm{mm} \times 30-\mathrm{m}$ capillary column with a 1- $\mu \mathrm{m}$ film of DB-WAX as the stationary phase. The GC carrier gas was helium at a column head pressure of approximately $4 \mathrm{psi}$. The column oven temperature was maintained at $70^{\circ} \mathrm{C}$ for $1 \mathrm{~min}$, then ramped to $110^{\circ} \mathrm{C}$ at $10^{\circ} / \mathrm{min}$, and held at $110^{\circ} \mathrm{C}$ for $5 \mathrm{~min}$. External standards in deionized water were used to quantify the results. Detection limits for this procedure were on the order of $0.06 \mathrm{ppm}$ in dilute exhaust.

\section{Mass Spectral Analyses}

Mass spectral analyses were also conducted on exhaust samples in an effort to determine exhaust hydrocarbons not identified by the previously described speciation methods. Sample collection was accomplished by drawing CVS-diluted exhaust through two tubes in series, each packed with a solid sorbent material. Figure 2 is a schematic drawing of the sampling system. 


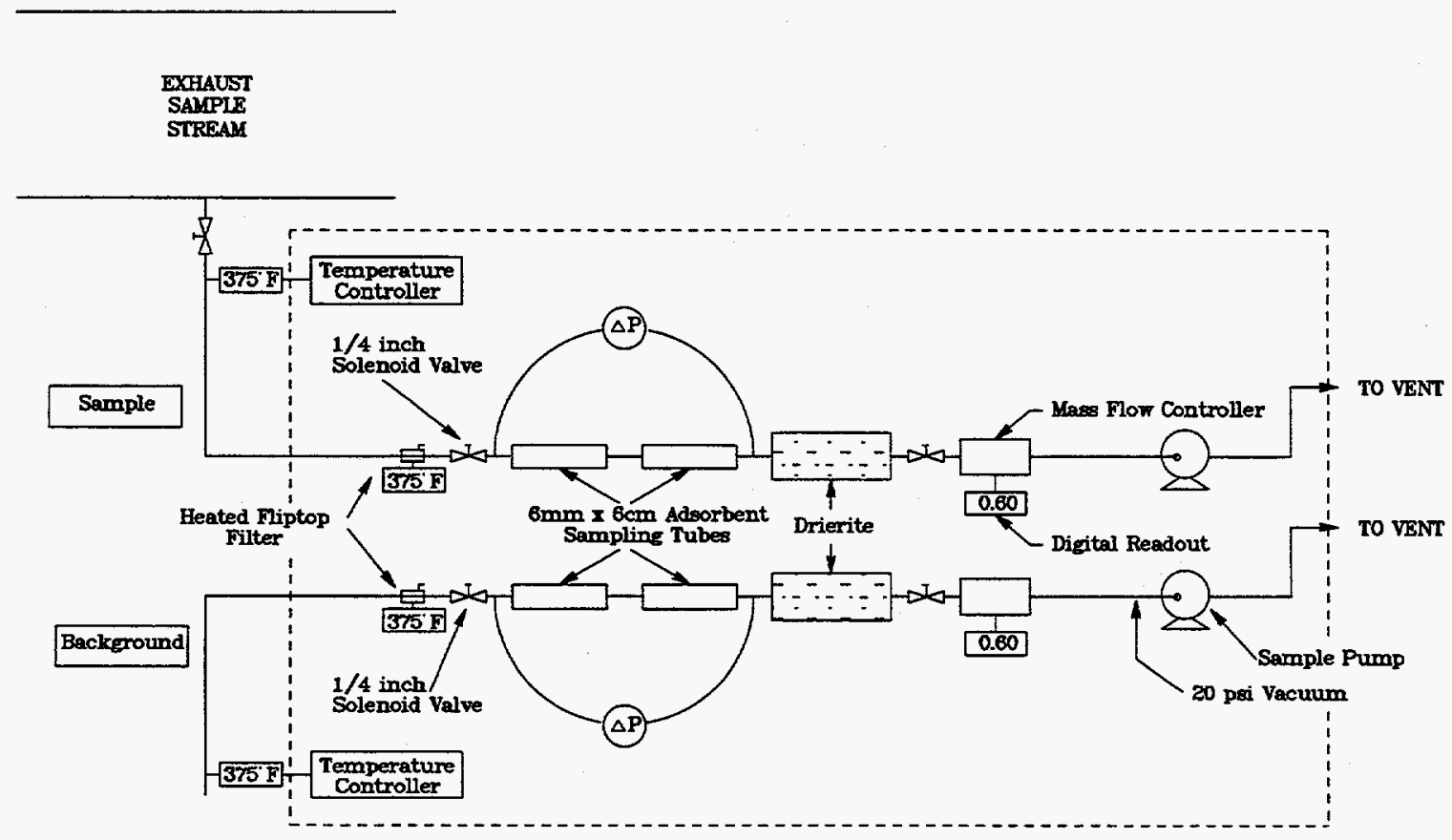

FIGURE 2. SCHEMATIC OF MASS SPECTRAL ANALYSES

SAMPLING SYSTEM 
Exhaust samples were collected continuously during the test cycle. The temperature of the sampling system up to the sorbent tubes is maintained at $375^{\circ} \mathrm{F}$. Before reaching the sorbent tubes, the sample passes through heated, flip-top filter holders fitted with glass fiber particulate filters. The sorbent tubes are borosilicate glass tubes packed with a proprietary sorbent material, which is held in place with small end plugs of silanized glass wool.

For analysis, the samples were thermally desorbed onto a GC column coupled to a quadruple MS and a FID. A thermal desorption autosampler is used to heat the sorbent tube to $300^{\circ} \mathrm{C}$ while helium, the inert carrier gas, purges the sample from the tube. The sample is subsequently caught on a cryogenically cooled trap maintained at $-100^{\circ} \mathrm{C}$. The tube is purged for 300 seconds. At this point the cryogenic trap is ballistically heated to $300^{\circ} \mathrm{C}$, introducing the sample onto the GC column. A splitless period of 30 seconds is used. To help in resolving the lighter compounds, the GC is cryogenically cooled at the start of the analysis, then ramped to the final set point. During the sample analysis period, the autosampler switches modes to further clean and condition the sorbent tube for future sampling by continuing to heat and purge the tube.

Hydrocarbons smaller than $\mathrm{C}_{6}$ are not collected by the solid sorbent material. However, the $\mathrm{C}_{1}$ to $\mathrm{C}_{5}$ compounds are readily identified by the three $\mathrm{GC}$ speciation methods described in Section II.E.2.

\section{VEHICLE TESTING}

\section{A. Baseline Testing with Gasoline}

To establish a baseline on the Chevrolet Lumina, an initial "check-out" FTP was conducted using the unleaded gasoline present in the vehicle when it was delivered. The results of this test are given in Table 7. A detailed computer printout of the emissions data is presented in Appendix A. The results of this test showed the vehicle emitted an unexpectedly high amount of $\mathrm{CO}$. Because of these results, the vehicle underwent a thorough diagnostic check. Although the "CHECK ENGINE" light was not illuminated, an error code was resident in the ECM memory. This code indicated an error in the operation of the IAC valve. The valve was inspected, appeared to be in proper working order, and was reinstalled.

The vehicle was refueled with RFG, the error code was cleared, and the vehicle was driven in urban traffic to ensure that the error code would not repeat. The vehicle was then driven over the UDDS three times as preconditioning for FTP testing. Throughout 15 miles of urban driving and 22.5 miles of operation on the chassis dynamometer, the IAC error code never reoccurred.

Duplicate FTPs were conducted on RFG to confirm proper vehicle operation and to establish baseline emissions levels for this program. Results of these tests are presented in Table 7. The repeatability of the two tests met the Auto/Oil program's test repeatability criteria. Detailed computer printouts of the emissions data are presented in Appendix B. For comparison purposes, FTP results from two other Luminas tested at SwRI (from NREL Report No. TP-421-5462) are also provided. Although the average CO emissions from these duplicate baseline tests were slightly above EPA standards, overall emissions seem to be representative 
of this model vehicle. These baseline results were approved by the NREL Technical Monitor before the program proceeded.

TABLE 7. INITIAL LUMINA TEST RESULTS WITH GASOLINE

(U.S. FTP CYCLE)

\begin{tabular}{|c|c|c|c|c|c|}
\hline Test Vehicle & Test No. & Test Fuel & $\begin{array}{c}\mathrm{HC} \\
\mathrm{g} / \mathrm{mile}\end{array}$ & $\begin{array}{c}\text { co } \\
\mathrm{g} / \mathrm{mile}\end{array}$ & $\begin{array}{c}\mathrm{NO}_{x} \\
g / \mathrm{mile}\end{array}$ \\
\hline '93 Lumina Checkout & CHECK-OUT & $\begin{array}{l}\text { unleaded gasoline } \\
\text { (as received) }\end{array}$ & 0.31 & 6.02 & 0.59 \\
\hline '93 Lumina Baseline & L-PH2-REF-R1 & Phase 2 RFG & 0.21 & 3.34 & 0.36 \\
\hline '93 Lumina Baseline ${ }^{a}$ & L-PH2-REF-R2 & Phase 2 RFG & 0.25 & 4.64 & 0.32 \\
\hline '91 Lumina Baseline & average of 2 tests & Howell EEE & 0.37 & 4.56 & 0.49 \\
\hline '92 Lumina Baseline ${ }^{b}$ & average of 3 tests & Howell EEE & 0.32 & 4.25 & 0.40 \\
\hline
\end{tabular}

\section{B. Testing with LPG}

The LPG conversion kit was installed on the vehicle without difficulty. At the recommendation of GM, the catalytic converter was replaced with one designed for use in an alcohol-fueled Lumina. Prior to installation, this catalyst was stabilized in a test cell as described in Section II.A. In addition, the UEGO sensor and comparator circuit needed to control fuel/air equivalence ratio were installed. The vehicle operated satisfactorily over the FTP at stoichiometry. However, lean operation of the vehicle was limited by poor driveability. In addition, rich operation of the vehicle was limited to a 1.15 equivalence ratio. Settings richer than this led to uncontrolled operation of the conversion kit. The fuel control system was able to maintain fuel/air equivalence ratios around desired set points throughout most portions of the FTP. However, the system would not maintain adequate fuel control during heavy accelerations and decelerations. In addition, the conversion kit operated extremely rich during FTP idles (1.18 1.20 air-fuel equivalence ratio) in all states of adjustment. SwRI contacted the kit manufacturer concerning this situation; however, attempts to change the idle performance of the conversion kit were unsuccessful. Testing on LPG proceeded smoothly, and repeatability criteria were met at all test conditions. Detailed computer printouts of the emissions results for these tests are provided in Appendix C.

\section{Testing with CNG}

The CNG conversion kit for this program shared many of its parts with the LPG kit. To configure the vehicle to operate on $\mathrm{CNG}$, the pressure regulator, feedback fuel control valve, and air/gas mixer from the LPG kit were replaced with components appropriate to operation on CNG. The vehicle operated satisfactorily over the FTP at stoichiometry and lean conditions. Rich operation of the vehicle was limited by poor driveability. The fuel control system was able to maintain fuel/air equivalence ratios around desired set points throughout most portions of the FTP. However, as was the case with LPG operation, the system would not maintain adequate fuel control during heavy accelerations and decelerations, and operated extremely rich at idle (1.18 1.20 air-fuel equivalence ratio) during FTP tests, even at stoichiometric and 
lean calibrations. SwRI also contacted the kit manufacturer concerning this situation; however, as was the case with LPG, attempts to change the idle performance of the conversion kit were unsuccessful.

Testing with CNG was temporarily delayed after only three tests were conducted. The cause of the delay was to ensure worker safety during CNG testing. A safety concern arose as a result of fuel tank failures in California and Minnesota involving CNG-fueled pickup trucks equipped with aluminum/fiberglass composite fuel cylinders. SwRI was fueling the CNG test vehicle from aluminum/fiberglass composite cylinders and felt it was prudent to remove these cylinders from service. Steel cylinders were obtained for CNG storage, and testing proceeded after a 2-week delay.

The CNG that remained in the aluminum/fiberglass composite cylinders was transferred to the steel cylinders. The test matrix had been interrupted between duplicate test runs with the vehicle operating at a $1.0 \mathrm{fuel} /$ air equivalence ratio without a catalyst. When the testing resumed, $\mathrm{HC}$ emissions results from the duplicate tests conducted before and after the delay differed significantly. A third test was conducted, and the results of the two tests conducted after the delay were within repeatability requirements for this program. There were no further test-to-test repeatability problems while operating the vehicle on CNG. Detailed computer printouts of the emissions results for these tests are provided in Appendix D.

\section{Testing with Reformulated Gasoline}

After the Lumina was restored to its original configuration, the UEGO sensor and comparator circuit used during testing on LPG and CNG were re-installed. Test L-PH2-1.0CK2 was conducted for comparison to baseline results to ensure proper operation of the vehicle and fuel control system. Emissions results of Test L-PH2-1.0-CK2 are compared to average baseline emissions in Table 8 and represent tests conducted 5 months apart. Emissions results were similar enough to meet the test-to-test repeatability criteria for this program and they confirmed proper vehicle operation. The test vehicle operated satisfactorily over all operating conditions and maintained desired fuel/air equivalence ratios over most operating conditions; however, when testing at lean conditions, the vehicle would operate near stoichiometry during accelerations due to fuel enrichment compensation. Testing with reformulated gasoline proceeded smoothly, and repeatability criteria were met at all test conditions. Detailed computer printouts of the emissions results are provided in Appendix $\mathrm{E}$.

TABLE 8. LUMINA FTP RESULTS COMPARED TO BASELINE

\begin{tabular}{||l|c|c|}
\hline \hline Test & Average of 2 baseline tests & L-PH2-1.0-CK2 \\
\hline FID HC $(\mathrm{g} / \mathrm{mi})$ & 0.23 & 0.25 \\
\hline CO $(\mathrm{g} / \mathrm{mi})$ & 3.99 & 4.64 \\
\hline NO $(\mathrm{g} / \mathrm{mi})$ & 0.34 & 0.32 \\
\hline $\begin{array}{l}\text { Hydrocarbon as measured with flame ionization detector calibrated } \\
\text { on propane }\end{array}$ \\
\hline
\end{tabular}




\section{E. Testing with Ethanol}

As received, the Chevrolet Corsica was calibrated to operate on ethanol. To prepare the vehicle for testing, an OEM 3-way catalyst designed for use in an alcohol-fueled Lumina was installed. This catalyst was the same one used in previous testing of LPG and CNG. In addition, a wide range oxygen sensor and the custom closed-loop fuel control system used in previous testing were installed on the vehicle. These systems appeared to operate properly when the test vehicle was driven over the UDDS. However, the vehicle did not start or idle well when the engine was at ambient temperature. An FTP test (C-ETH-1.0-CK1) was conducted to determine the extent of the driveability problems. Emissions results for this test are given in Table 9. Engine cranking was necessary for approximately 8 seconds before engine firing occurred. Cold-start idle was rough, and the engine stumbled and backfired during the first acceleration of the FTP. However, once the vehicle warmed up and entered closed-loop operation, driveability was satisfactory. This vehicle was originally calibrated to operate on M85, and E100 has a much lower volatility; therefore, it was necessary to modify the cold-start calibration to provide additional fuel during starting and warmup. Using the CDM on the vehicle, open-loop acceleration fuel enrichment was increased until the vehicle operated smoothly over the complete FTP cycle. The CDM is a dash-top computer that contains all the vehicle calibrations for ethanol and methanol operation. FTP emissions results with the final calibration (C-ETH-1.0-CK8) are also given in Table 9. Although the calibration modifications resulted in increased mass emissions from the vehicle, they were necessary to maintain satisfactory driveability over the FTP.

TABLE 9. INITIAL CORSICA TEST RESULTS WITH ETHANOL (U.S. FTP CYCLE)

\begin{tabular}{||l|c|c||}
\hline Test Number & C-ETH-1.0-CK1 & C-ETH-1.0-CK8 \\
\hline FID HC $^{\mathbf{a}}$ (g/mi) & 0.577 & 0.895 \\
\hline $\mathbf{C O}(\mathbf{g} / \mathrm{mi})$ & 1.761 & 4.288 \\
\hline $\mathrm{NO}_{\mathbf{x}}$ (g/mi) & 0.494 & 0.571 \\
\hline Fuel Economy (mpg) & 11.38 & 11.36 \\
\hline $\begin{array}{l}\text { a Hydrocarbon as measured with flame ionization detector calibrated on } \\
\text { propane; not corrected for differing response to alcohols. }\end{array}$ \\
\hline
\end{tabular}

Testing with ethanol was initiated; however, after the first test (C-ETH-1.0-C1), the fuel pump failed and had to be replaced. When the testing resumed, $\mathrm{HC}$ emissions results from the duplicate tests (C-ETH-1.0-C1 and C-ETH-1.0-C2) differed significantly. A third test (C-ETH1.0-C3) was conducted and the results of that test and test C-ETH-1.0-C2 were within repeatability requirements for this program. These data are presented in Table 10. No further repeatability problems were encountered during testing with ethanol. 
TABLE 10. FTP RESULTS BEFORE AND AFTER FUEL PUMP REPLACEMENT

\begin{tabular}{|c|c|c|c|c|c|c|}
\hline & \multicolumn{3}{|c|}{ TEST } & \multicolumn{3}{|c|}{ REPEATABILITY RATIO } \\
\hline & C-ETH-1.0-C1 & C-ETH-1.0-C2 & C-ETH-1.0-C3 & $\mathrm{E} 1$ to $\mathrm{E2}$ & E2 to E3 & LIMIT \\
\hline TEST DATE & $06 / 21 / 94$ & $06 / 24 / 94$ & $06 / 27 / 94$ & & & \\
\hline ODOMETER (miles) & 14,871 & 14,903 & 14,921 & & & \\
\hline FID HC $(\mathrm{g} / \mathrm{mi})$ & 1.009 & 0.702 & 0.861 & 1.44 & 1.23 & 1.33 \\
\hline $\mathrm{CO}(\mathrm{g} / \mathrm{mi})$ & 4.134 & 3.284 & 3.719 & 1.26 & 1.13 & 1.70 \\
\hline $\mathrm{NO}_{x}(\mathrm{~g} / \mathrm{mi})$ & 0.479 & 0.557 & 0.542 & 1.16 & 1.03 & 1.29 \\
\hline $\begin{array}{l}\text { a Total hydrocarbor } \\
\text { corrected for diffe } \\
\text { Repeatability rati } \\
\text { ratio criteria per }\end{array}$ & $\begin{array}{l}\text { Is as measured } \\
\text { ring response to } \\
\text { is ratio of the la } \\
\text { SAE Paper } 9203\end{array}$ & $\begin{array}{l}\text { with flame ioniz } \\
\text { alcohols. } \\
\text { arger to the sme } \\
319 \text {. }\end{array}$ & $\begin{array}{l}\text { on detector c } \\
\text { value of the }\end{array}$ & $\begin{array}{l}\text { rated on } \\
\text { Deat pair. }\end{array}$ & $\begin{array}{l}\text { ropane; n } \\
\text { Repeatabi }\end{array}$ & \\
\hline
\end{tabular}

After conducting tests at stoichiometry with and without the catalyst, the vehicle was tested at lean conditions. With the exception of poor vehicle driveability, no problems were encountered during the lean-operation tests. Following testing at lean conditions, preparations were made to test the vehicle under fuel-rich conditions. However, the CDM on this vehicle is apparently equipped with a fail-safe device that prohibits extremely rich operation. The SwRI custom fuel control system was not able to adjust the control system to force the vehicle to operate rich. In an effort to get the vehicle to operate at rich conditions, SwRI consulted with General Motors; however, no obvious solutions were found. In an effort to force the vehicle to operate fuel-rich, the coolant temperature sensor was bypassed with the signal from an identical sensor placed in ice water. This condition forced the vehicle to use a cold-temperature calibration that provided additional enrichment and to remain in open-loop operation longer. However, the resulting operation was only slightly richer than stoichiometry. In addition, vehicle driveability during open-loop operation was less than satisfactory. Idle was rough, and the engine would stumble and backfire during accelerations. However, the vehicle was able to meet repeatability criteria for all test conditions. Detailed computer printouts of the emissions results for individual tests are provided in Appendix F.

\section{F. Testing with Methanol}

After testing on ethanol, the fuel system was drained and flushed with methanol, and the vehicle's calibration was changed to the one specified by the University of Tennessee for operation on methanol. However, the vehicle did not start or idle well when the engine was at ambient temperatures, and the vehicle could not be driven satisfactorily over the FTP. Coldstart idle was rough, the engine stumbled and backfired, and the vehicle could not follow the FTP trace during heavy acceleration in the open-loop fuel-control mode. While attempting to correct the open-loop performance of the vehicle, the CDM malfunctioned, and the vehicle would not operate properly. After repairs were made, the open-loop calibration of the vehicle was adjusted. The vehicle operated smoothly over the FTP cycle, except during the first 60 seconds of Bag 1. In this case, the vehicle was in open-loop fuel control and would stumble and 
sometimes backfire slightly during accelerations. Attempts to improve the open-loop fuel calibration of the vehicle were unsuccessful. After consulting with the NREL Project Officer, it was determined that further calibration of the vehicle was beyond the scope of this program.

As with operation on ethanol, the vehicle could not be adjusted to operate rich as planned. As was done during testing on ethanol, the coolant temperature sensor was bypassed with the signal from an identical sensor placed in ice water in an effort to make the vehicle operate fuelrich. This condition forced the vehicle to use a cold-temperature calibration, which provided additional enrichment, and to remain in open-loop operation longer. However, as was the case with ethanol, the resulting operation was only slightly richer than stoichiometry and vehicle driveability during open-loop operation was less than satisfactory. Idle was rough, and the engine would stumble and backfire during accelerations. The vehicle was able to meet repeatability criteria for all test conditions. Detailed computer printouts of the emissions results for individual tests are included in Appendix G.

\section{G. Fuel/Air Equivalence Ratios Achieved During Testing}

For each fuel, the fuel control system was tuned to the desired fuel/air equivalence ratio set point by operating the vehicle on the chassis dynamometer at a steady-state condition of 50 $\mathrm{mph}$. Actual road load was set at $6.5 \mathrm{hp}$ for the Lumina and $4.5 \mathrm{hp}$ for the Corsica. These road loads correspond to the dynamometer setting during FTP testing. The fuel/air equivalence ratio set points used during testing with each fuel are shown in Table 11. As previously noted, target equivalence ratios were not achieved for all test conditions. Lean operation on LPG, ethanol, and methanol was limited by poor driveability, as was rich operation on CNG. Rich operation on LPG, ethanol, and methanol was limited by the capabilities of the vehicles' fuel-control systems.

TABLE 11 - TEST FUELIAIR EQUIVALENCE RATIOS

\begin{tabular}{||c||c|c|c|c|c||}
\hline \multicolumn{6}{|c|}{ Fuel/Air Equivalence Ratio } \\
\hline Desired & LPG & CNG & RFG & EtOH & MtOH \\
\hline \hline 0.8 & 0.83 & 0.80 & 0.81 & 0.86 & 0.90 \\
\hline 1.0 & 1.01 & 0.99 & 0.99 & 1.01 & 1.00 \\
\hline 1.2 & 1.15 & 1.10 & 1.20 & 1.05 & 1.05 \\
\hline
\end{tabular}

\section{TEST RESULTS AND DISCUSSION}

This section includes the presentation and discussion of results of testing with all the fuels. Regulated exhaust emissions, toxic exhaust emissions, speciated exhaust emissions, potential ozone formation, and mass spectral analyses results are provided. 


\section{A. Regulated Exhaust Emissions}

Tables 12 and 13 show NMOG, $\mathrm{CO}$, and $\mathrm{NO}_{\mathrm{x}}$ exhaust emissions for each of the five fuels as a function of operating conditions without and with the vehicle catalyst in place, respectively. Also contained in Tables 12 and 13 are values for both the average THC mass emissions determined by hydrocarbon speciation (THC/GC) and by a flame ionization detector (THC/FID). The THC values were based on fuel density and fuel weight fractions of carbon, hydrogen, and oxygen, but did not take into consideration the FID response factors for MtOH or EtOH. Agreement between THC mass determined by the two methods was found to be good, with a maximum difference of $11.5 \%$ observed for a single test. Most tests showed differences of less than 5\%. Although the Lumina did not meet CO tailpipe emissions standards with any fuel, emissions measured from the stock vehicle operating on reformulated gasoline are consistent with previously published emissions data for this model vehicle. ${ }^{(4)}$ The Corsica was an experimental vehicle operating on neat alcohols and was not required to meet certification standards. Although $\mathrm{CO}$ and $\mathrm{NO}_{\mathrm{x}}$ emissions levels from the alcohol fuels were similar to those from other fuels, NMOG emissions were 3 to 5 times higher at stoichiometric conditions. In addition, none of the alternative fuel configurations were optimized to provide the lowest possible tailpipe emissions; therefore, fuel-to-fuel comparisons of absolute FTP results are inconclusive. However, trends between rich, stoichiometric, and lean operation are observable for all fuels.

At stoichiometric operation, catalyst efficiencies for $\mathrm{THC}, \mathrm{CO}$, and $\mathrm{NO}_{\mathrm{x}}$ were greater than $75 \%$ for all fuels except for the alcohols, where $\mathrm{NO}_{\mathrm{x}}$ efficiencies were less than $40 \%$. While operating on LPG and reformulated gasoline at rich conditions without a catalyst, HC emissions were about twice as high and $\mathrm{CO}$ emissions were approximately 5 to 7 times as high as at stoichiometric operation without a catalyst. THC emissions during rich operation on CNG without a catalyst were about 4 times higher and $\mathrm{CO}$ emissions about 11 times higher than during stoichiometric operation. The high $\mathrm{HC}$ emissions indicate that severe engine misfiring occurred with CNG at rich conditions. While operating on ethanol and methanol, fuel-rich conditions were only slightly richer than at stoichiometric conditions. In addition, the method of obtaining rich operation used a calibration that was completely different from the stoichiometric conditions. As a result, THC emissions without a catalyst increased less than $10 \%$ from stoichiometric conditions while operating on ethanol and decreased by about $10 \%$ while operating on methanol. The slight decrease in THC emissions on methanol can be attributed to less stumbling and backfiring during rich operation than at the stoichiometric condition. $\mathrm{CO}$ emissions without a catalyst were about $40 \%$ and $20 \%$ higher during rich operation than at stoichiometric conditions with ethanol and methanol, respectively. 
TABLE 12. SUMMARY OF AVERAGE FTP EMISSIONS WITHOUT CATALYST

\begin{tabular}{|c|c|c|c|c|c|}
\hline OPERATING CONDITION & \multicolumn{5}{|c|}{ FUEL-LEAN } \\
\hline VEHICLE & \multicolumn{3}{|c|}{ LUMINA } & \multicolumn{2}{|c|}{ CORSICA } \\
\hline FUEL & LPG & CNG & RFG & EtOH & MtOH \\
\hline TEST EQUIVALENCE RATIO & 0.83 & 0.80 & 0.81 & 0.86 & 0.90 \\
\hline NMOG (grams/mile) & 4.89 & 0.46 & 11.31 & 10.84 & 8.64 \\
\hline CO (grams/mile) & 11.17 & 9.63 & 9.27 & 9.03 & 9.28 \\
\hline $\mathrm{NO}_{x}$ (grams/mile) & 1.28 & 1.50 & 1.34 & 0.60 & 0.55 \\
\hline THC/FID (grams/mile) & 5.52 & 2.47 & 11.17 & 10.95 & 8.96 \\
\hline THC/GC (grams/mile) & 4.98 & 2.59 & 11.40 & 10.99 & 8.66 \\
\hline OPERATING CONDITION & \multicolumn{5}{|c|}{ STOICHIOMETRIC } \\
\hline VEHICLE & \multicolumn{3}{|c|}{ LUMINA } & \multicolumn{2}{|c|}{ CORSICA } \\
\hline FUEL & LPG & CNG & $\mathbf{R F G}$ & $\mathrm{ELOH}$ & MtOH \\
\hline TEST EQUIVALENCE RATIO & 1.01 & 0.99 & 0.99 & 1.01 & 1.00 \\
\hline NMOG (grams/mile) & 1.84 & 0.55 & 2.41 & 5.20 & 5.42 \\
\hline CO (grams/mile) & 20.96 & 14.53 & 17.30 & 15.67 & 14.30 \\
\hline $\mathrm{NO}_{x}$ (grams/mile) & 2.27 & 1.17 & 2.29 & 0.75 & 0.57 \\
\hline THC/FID (grams/mile) & 1.95 & 3.19 & 2.51 & 5.25 & 5.52 \\
\hline THC/GC (grams/mile) & 1.94 & 3.23 & 2.48 & 5.36 & 5.45 \\
\hline OPERATING CONDITION & \multicolumn{5}{|c|}{ FUEL-RICH } \\
\hline VEHICLE & \multicolumn{3}{|c|}{ LUMINA } & \multicolumn{2}{|c|}{ CORSICA } \\
\hline FUEL. & LPG & CNG & $\mathbf{R F G}$ & EtOH & MtOH \\
\hline TEST EQUIVALENCE RATIO & 1.15 & 1.10 & 1.20 & 1.05 & 1.05 \\
\hline NMOG (grams/mile) & 2.50 & 1.82 & 3.64 & 5.74 & 4.78 \\
\hline CO (grams/mile) & 100.1 & 103.2 & 124.4 & 21.50 & 17.49 \\
\hline $\mathrm{NO}_{x}$ (grams/mile) & 0.65 & 0.39 & 0.78 & 0.99 & 1.41 \\
\hline THC/FID (grams/mile) & 2.92 & 12.25 & 4.14 & 5.86 & 4.78 \\
\hline THC/GC (grams/mile) & 2.87 & 12.36 & 4.01 & 5.91 & 4.81 \\
\hline
\end{tabular}


TABLE 13. SUMMARY OF AVERAGE FTP EMISSIONS WITH CATALYST

\begin{tabular}{|c|c|c|c|c|c|}
\hline OPERATING CONDITION & \multicolumn{5}{|c|}{ FUEL-LEAN } \\
\hline VEHICLE & \multicolumn{3}{|c|}{ LUMINA } & \multicolumn{2}{|c|}{ CORSICA } \\
\hline FUEL. & LPG & CNG & RFG & EtOH & MtOH \\
\hline TEST EQUIVALENCE RATIO & 0.83 & 0.80 & 0.81 & 0.86 & 0.90 \\
\hline NMOG (grams/mile) & 0.49 & 0.07 & 0.19 & 0.98 & 0.65 \\
\hline CO (grams/mile) & 2.41 & 1.16 & 4.24 & 2.67 & 1.86 \\
\hline $\mathrm{NO}_{\mathrm{x}}$ (grams/mile) & 1.36 & 1.11 & 1.03 & 0.57 & 0.38 \\
\hline THC/FID (grams/mile) & 0.56 & 1.01 & 0.24 & 1.00 & 0.65 \\
\hline THC/GC (grams/mile) & 0.56 & 1.03 & 0.25 & 1.03 & 0.66 \\
\hline OPERATING CONDITION & \multicolumn{5}{|c|}{ STOICHIOMETRIC } \\
\hline VEHICLE & \multicolumn{3}{|c|}{ LUMINA } & \multicolumn{2}{|c|}{ CORSICA } \\
\hline FUEL & LPG & CNG & $\mathbf{R F G}$ & EtoH & MtOH \\
\hline TEST EQUIVALENCE RATIO & 1.01 & 0.99 & 0.99 & 1.01 & 1.00 \\
\hline NMOG (grams/mile) & 0.17 & 0.05 & 0.19 & 0.93 & 0.63 \\
\hline CO (grams/mile) & 4.48 & 3.59 & 4.20 & 3.50 & 2.35 \\
\hline $\mathrm{NO}_{\mathrm{x}}$ (grams/mile) & 0.60 & 0.20 & 0.34 & 0.55 & 0.36 \\
\hline THC/FID (grams/mile) & 0.29 & 0.84 & 0.25 & 0.96 & 0.65 \\
\hline THC/GC (grams/mile) & 0.29 & 0.86 & 0.22 & 1.00 & 0.65 \\
\hline OPERATING CONDITION & \multicolumn{5}{|c|}{ FUEL-RICH } \\
\hline VEHICLE & \multicolumn{3}{|c|}{ LUMINA } & \multicolumn{2}{|c|}{ CORSICA } \\
\hline FUEL & LPG & CNG & RFG & EtOH & MtOH \\
\hline TEST EQUIVALENCE RATIO & 1.15 & 1.10 & 1.20 & 1.05 & 1.05 \\
\hline NMOG (grams/mile) & 1.78 & 0.45 & 3.59 & 1.16 & 0.79 \\
\hline CO (grams/mile) & 94.75 & 39.12 & 124.57 & 4.75 & 3.35 \\
\hline $\mathrm{NO}_{\mathbf{x}}$ (grams/mile) & 0.03 & 0.02 & 0.36 & 0.40 & 0.83 \\
\hline THC/FID (grams/mile) & 2.33 & 9.15 & 4.04 & 1.30 & 0.80 \\
\hline THC/GC (grams/mile) & 2.33 & 9.27 & 3.59 & 1.28 & 0.81 \\
\hline
\end{tabular}


For fuel-rich conditions operating on the gaseous fuels and on reformulated gasoline, $\mathrm{NO}_{\mathrm{x}}$ emissions without a catalyst were reduced by more than half from stoichiometric levels. The high levels of $\mathrm{CO}$ in the exhaust (greater than $100 \mathrm{~g} / \mathrm{mi}$ for all fuels) during rich operation allowed for nearly all the $\mathrm{NO}_{\mathrm{x}}$ to be reduced across the catalyst; however, the lack of excess oxygen in the exhaust resulted in generally poor catalyst efficiencies for THC and CO with LPG and RFG. Because the achievable fuel-rich equivalence ratio was limited to 1.10 while operating on CNG, THC and CO catalyst efficiencies were slightly better than LPG and RFG. Given the unusual calibration of the Corsica while operating at rich conditions on the alcohol fuels, engine-out $\mathrm{NO}_{\mathrm{x}}$ emissions were 1.3 times higher while operating on ethanol and were nearly 2.5 times higher while operating on methanol than at stoichiometric conditions. Catalyst efficiencies during rich operation of the Corsica were better than at stoichiometric operation, with greater than $80 \%$ efficiency for $\mathrm{THC}$ and $\mathrm{CO}$ and about $50 \%$ reduction of $\mathrm{NO}_{\mathrm{x}}$ on both alcohol fuels.

At lean conditions without a catalyst, CO emissions from LPG, RFG, ethanol, and methanol fuels were approximately half of those produced during stoichiometric operation. However, due to lean misfire on these fuels, THC emissions were about 2 to 4 times higher at lean conditions than at stoichiometric conditions. Lean $\mathrm{NO}_{\mathrm{x}}$ emissions were nearly half of stoichiometric levels during operation on LPG and RFG and were almost $20 \%$ lower than at stoichiometric conditions while operating on the alcohol fuels. While operating on CNG, combustion appeared stable at lean conditions. This resulted in lower THC and CO emissions without a catalyst compared to stoichiometric operation, and in slightly elevated $\mathrm{NO}_{\mathrm{x}}$ levels. The abundance of excess oxygen in the exhaust stream at lean conditions allowed for good conversion of THC and $\mathrm{CO}$ in the catalyst while operating on all fuels. However, excess oxygen also resulted in poor catalytic control of $\mathrm{NO}_{\mathrm{x}}$. Lean operation on LPG gave a slight increase in average $\mathrm{NO}_{\mathrm{x}}$ emissions across the catalyst.

\section{B. Toxic Exhaust Emissions}

Emissions of air toxics are presented in Table 14 for all three fuel/air equivalence ratios investigated. There was speculation that the high level of toxic emissions from the alcohol fuels might have been due to the poor operation of the Corsica during open-loop operation in Bags 1 and 3 of the FTP. Since the Corsica operated satisfactorily during hot stabilized operation, toxic emissions measured in Bag 2 are also presented in Table 14 for comparison.

In general, formaldehyde accounted for $65 \%$ to $80 \%$ of toxic mass emissions while operating on LPG and CNG at stoichiometric and fuel-lean conditions. Under the same conditions, acetaldehyde accounted for about $25 \%$ of toxic emissions from LPG. However, at fuel-rich conditions a large portion of tailpipe toxic emissions from the gaseous fuels unexpectedly consisted of benzene, which accounted for $54 \%$ and $36 \%$ of toxic emissions from LPG and CNG, respectively. The origin of the benzene emissions is currently unknown. Toxic emissions from reformulated gasoline consisted primarily of benzene ( $65 \%)$, formaldehyde $(\sim 20 \%)$, and 1,3-butadiene ( $10 \%)$. About $90 \%$ of after-catalyst toxic emissions from ethanol were acetaldehyde and $10 \%$ were formaldehyde. Nearly all of the toxic emissions from operation on methanol were from formaldehyde. 
TABLE 14 - AVERAGE FTP TOXIC EMISSIONS RATES

\begin{tabular}{|c|c|c|c|c|c|c|c|c|c|c|c|}
\hline \multicolumn{12}{|c|}{ FTP } \\
\hline \multirow{2}{*}{$\begin{array}{l}\text { Operating } \\
\text { Condition }\end{array}$} & \multirow[b]{2}{*}{ Compound } & \multicolumn{5}{|c|}{ Without Catalyst } & \multicolumn{5}{|c|}{ With Catalyst } \\
\hline & & LPG & CNG & $\mathbf{R F G}$ & EtoH & MtOH & LPG & CNG & RFG & EtOH & MtOH \\
\hline \multirow[t]{2}{*}{ Lean } & \begin{tabular}{|l} 
Formaldehyde \\
Acetaldehyde \\
Benzene \\
1,3-Butadiene
\end{tabular} & $\begin{array}{r}199.8 \\
38.9 \\
0.7 \\
3.1 \\
\end{array}$ & $\begin{array}{r}92.3 \\
4.2 \\
0.5 \\
0.8\end{array}$ & $\begin{array}{r}485.0 \\
82.4 \\
168.5 \\
61.7\end{array}$ & \begin{tabular}{r|}
318.2 \\
1131.2 \\
1.8 \\
1.0 \\
\end{tabular} & \begin{tabular}{r|}
736.1 \\
6.3 \\
1.4 \\
0.6 \\
\end{tabular} & $\begin{array}{l}2.4 \\
0.7 \\
0.0 \\
0.1\end{array}$ & $\begin{array}{r}1.2 \\
0.1 \\
0.1 \\
<0.1\end{array}$ & $\begin{array}{l}2.7 \\
0.9 \\
7.2 \\
1.2\end{array}$ & $\begin{array}{r}6.1 \\
52.5 \\
0.6 \\
0.2\end{array}$ & $\begin{array}{r}23.9 \\
0.1 \\
0.1 \\
<0.1\end{array}$ \\
\hline & Total Toxics & 242.5 & 97.8 & 797.6 & 1452.2 & 744.4 & 3.2 & 1.5 & 11.9 & 59.3 & 24.2 \\
\hline \multirow[t]{2}{*}{ Stoich. } & \begin{tabular}{|l} 
Formaldehyde \\
Acetaldehyde \\
Benzene \\
1,3-Butadiene
\end{tabular} & $\begin{array}{r}53.1 \\
17.7 \\
0.6 \\
1.8\end{array}$ & $\begin{array}{r}70.4 \\
3.7 \\
0.5 \\
0.6\end{array}$ & $\begin{array}{l}97.3 \\
18.3 \\
63.4 \\
21.3\end{array}$ & $\begin{array}{r}166.1 \\
478.1 \\
1.6 \\
0.7\end{array}$ & \begin{tabular}{r|}
409.3 \\
8.7 \\
1.4 \\
0.1 \\
\end{tabular} & $\begin{array}{l}1.0 \\
0.4 \\
0.1 \\
0.1\end{array}$ & $\begin{array}{l}0.9 \\
0.1 \\
0.2 \\
0.1\end{array}$ & $\begin{array}{l}2.7 \\
0.8 \\
7.9 \\
1.1\end{array}$ & $\begin{array}{r}5.3 \\
66.2 \\
0.7 \\
0.1\end{array}$ & $\begin{array}{r}24.4 \\
0.9 \\
0.1 \\
<0.1\end{array}$ \\
\hline & Total Toxics & 73.2 & 75.2 & 200.3 & 646.5 & 419.5 & 1.5 & 1.3 & 12.4 & 72.4 & 25.3 \\
\hline \multirow[t]{2}{*}{ Rich } & $\begin{array}{l}\text { Formaldehyde } \\
\text { Acetaldehyde } \\
\text { Benzene } \\
\text { 1,3-Butadiene } \\
\end{array}$ & $\begin{array}{r}52.7 \\
10.3 \\
0.7 \\
1.6 \\
\end{array}$ & $\begin{array}{r}137.0 \\
7.9 \\
0.8 \\
0.4 \\
\end{array}$ & $\begin{array}{r}53.8 \\
9.3 \\
120.0 \\
18.2 \\
\end{array}$ & $\begin{array}{r}172.4 \\
458.4 \\
1.6 \\
0.6 \\
\end{array}$ & \begin{tabular}{|r|}
343.8 \\
1.9 \\
1.6 \\
0.4 \\
\end{tabular} & $\begin{array}{l}1.6 \\
1.2 \\
3.5 \\
0.3 \\
\end{array}$ & $\begin{array}{r}1.5 \\
0.3 \\
1.0 \\
<0.1 \\
\end{array}$ & $\begin{array}{r}20.5 \\
20.4 \\
126.3 \\
18.7 \\
\end{array}$ & $\begin{array}{r}7.2 \\
63.4 \\
1.0 \\
0.3 \\
\end{array}$ & $\begin{array}{r}29.7 \\
0.1 \\
0.2 \\
<0.1 \\
\end{array}$ \\
\hline & Total Toxics & 65.2 & 146.1 & 201.3 & 633.1 & 347.7 & 6.5 & 2.8 & 185.9 & 71.8 & 30.1 \\
\hline \multicolumn{12}{|c|}{ Bag 2} \\
\hline \multirow{2}{*}{$\begin{array}{l}\text { Operating } \\
\text { Condition }\end{array}$} & \multirow[b]{2}{*}{ Compound } & \multicolumn{5}{|c|}{ Without Catalyst } & \multicolumn{5}{|c|}{ With Catalyst } \\
\hline & & LPG & CNG & $\mathbf{R F G}$ & EtOH & MtOH & LPG & CNG & RFG & EtOH & MtOH \\
\hline \multirow[t]{2}{*}{ Lean } & $\begin{array}{l}\text { Formaldehyde } \\
\text { Acetaldehyde } \\
\text { Benzene } \\
\text { 1,3-Butadiene } \\
\end{array}$ & $\begin{array}{r}226.0 \\
42.7 \\
0.9 \\
3.6 \\
\end{array}$ & $\begin{array}{r}110.9 \\
4.9 \\
0.3 \\
0.7 \\
\end{array}$ & $\begin{array}{r}641.3 \\
97.7 \\
210.7 \\
73.9 \\
\end{array}$ & \begin{tabular}{|r|}
383.1 \\
1465.2 \\
1.6 \\
0.8 \\
\end{tabular} & \begin{tabular}{|r|}
887.6 \\
7.7 \\
1.4 \\
0.5 \\
\end{tabular} & $\begin{array}{r}0.2 \\
<0.1 \\
<0.1 \\
<0.1 \\
\end{array}$ & $\begin{array}{r}0.4 \\
0.1 \\
0.2 \\
<0.1 \\
\end{array}$ & $\begin{array}{r}0.4 \\
0.3 \\
1.2 \\
<0.1 \\
\end{array}$ & $\begin{array}{r}0.5 \\
0.7 \\
<0.1 \\
<0.1 \\
\end{array}$ & $\begin{array}{r}1.8 \\
0.1 \\
<0.1 \\
<0.1 \\
\end{array}$ \\
\hline & Total Toxics & 273.1 & 116.9 & 1023.7 & 1850.7 & 897.2 & 0.2 & 0.7 & 1.9 & 1.2 & 1.8 \\
\hline \multirow[t]{2}{*}{ Stoich. } & $\begin{array}{l}\text { Formaldehyde } \\
\text { Acetaldehyde } \\
\text { Benzene } \\
\text { 1,3-Butadiene } \\
\end{array}$ & $\begin{array}{r}41.7 \\
20.5 \\
0.6 \\
2.0 \\
\end{array}$ & $\begin{array}{r}88.1 \\
4.6 \\
0.5 \\
0.5 \\
\end{array}$ & $\begin{array}{r}110.5 \\
20.3 \\
67.3 \\
22.5 \\
\end{array}$ & $\begin{array}{r}175.3 \\
510.5 \\
1.4 \\
0.5 \\
\end{array}$ & $\begin{array}{r}393.8 \\
9.0 \\
1.4 \\
<0.1 \\
\end{array}$ & $\begin{array}{r}0.2 \\
0.1 \\
0.1 \\
<0.1 \\
\end{array}$ & $\begin{array}{r}0.2 \\
<0.1 \\
0.2 \\
0.1 \\
\end{array}$ & $\begin{array}{r}<0.1 \\
0.1 \\
<0.1 \\
<0.1 \\
\end{array}$ & $\begin{array}{r}0.5 \\
0.7 \\
0.4 \\
<0.1 \\
\end{array}$ & $\begin{array}{r}1.8 \\
<0.1 \\
<0.1 \\
<0.1\end{array}$ \\
\hline & Total Toxics & 64.8 & 93.7 & 220.6 & 687.7 & 404.1 & 0.4 & 0.5 & 0.1 & 1.5 & 1.9 \\
\hline \multirow[t]{2}{*}{ Rich } & \begin{tabular}{|l} 
Formaldehyde \\
Acetaldehyde \\
Benzene \\
1,3-Butadiene \\
\end{tabular} & $\begin{array}{r}56.4 \\
11.1 \\
0.5 \\
1.7 \\
\end{array}$ & $\begin{array}{r}161.4 \\
9.1 \\
0.9 \\
0.4 \\
\end{array}$ & $\begin{array}{r}58.5 \\
9.8 \\
136.1 \\
20.0 \\
\end{array}$ & $\begin{array}{r}162.3 \\
441.6 \\
1.3 \\
0.4 \\
\end{array}$ & $\begin{array}{r}326.4 \\
1.7 \\
1.7 \\
0.3 \\
\end{array}$ & $\begin{array}{l}0.1 \\
0.3 \\
3.9 \\
0.3 \\
\end{array}$ & $\begin{array}{r}0.1 \\
0.1 \\
1.1 \\
<0.1 \\
\end{array}$ & $\begin{array}{r}17.6 \\
24.3 \\
144.1 \\
21.0 \\
\end{array}$ & $\begin{array}{r}0.2 \\
0.6 \\
0.3 \\
<0.1 \\
\end{array}$ & $\begin{array}{r}1.7 \\
<0.1 \\
0.1 \\
<0.1 \\
\end{array}$ \\
\hline & Total Toxics & 69.7 & 171.7 & 224.4 & 605.6 & 330.1 & 4.5 & 1.3 & 207.0 & 1.1 & 1.8 \\
\hline
\end{tabular}


During rich operation on reformulated gasoline and the gaseous fuels, more benzene emissions were observed with the catalyst than without it. Although the origin of the additional benzene is unknown, it is speculated that thermal cracking and recombination of hydrocarbons within the catalyst is responsible for the increase. However, additional research is needed in this matter. When operating on RFG at rich conditions, an increase in acetaldehyde emissions across the catalyst was also observed, while at the same time formaldehyde emissions were lower. This situation could possibly be the result of partial oxidation in the oxygen-lean exhaust; however, the formation mechanism responsible for a net increase in acetaldehyde and a net decrease in formaldehyde across the catalyst is unknown.

\section{Potential Ozone Formation}

In order to allow for a comparison of emissions on the basis of ozone-forming potential, CARB has published a list of MIRs for a number of VOCs, defined as grams of ozone per gram of specific VOC emitted. These MIRs are given in Appendix $\mathrm{H}^{(5)}$, and were either estimated by CARB or derived from smog chamber experiments based on a Los Angeles atmospheric mix at VOC-limited ozone conditions. Following CARB methodology, a comparison of the ozoneforming potential on a gram per mile basis for each fuel is achieved by multiplying the MIR for each VOC by the emission rate of that VOC. The summation of these products yields the ozoneforming potential for a specific fuel [i.e., $\left.\mathrm{g} \mathrm{O}_{3} / \mathrm{mi}=\sum\left(\mathrm{VOC}_{\mathrm{i}} \times \mathrm{MIR}_{\mathrm{f}}\right)\right]{ }^{(5)}$

Presented in Table 15 is the potential mass of ozone formed by hydrocarbon emissions from each fuel for the entire FTP and for the Bag 2 segment of the FTP. During stoichiometric operation with a catalyst, the mass of potential ozone formed from CNG was $90 \%$ less, from LPG $68 \%$ less, and from methanol $23 \%$ less than from reformulated gasoline. FTP ozone forming potential of ethanol during stoichiometric operation with a catalyst was $150 \%$ more than with RFG, primarily because of unburned ethanol and ethylene in the exhaust during Bag 1 open-loop operation. Although the catalyst-out potential ozone formation of the alcohol fuels was higher than the gaseous fuels and RFG, the data are not directly comparable due to the cold-start calibration difficulties encountered with the Corsica. However, during hot, stabilized running (Bag 2), where the Corsica operated properly, the data show that the four alternative fuels have similar levels of ozone-forming potential with the vehicle catalyst in place.

TABLE 15 - POTENTIAL OZONE FORMATION (MG/MILE)

\begin{tabular}{|c|c|c|c|c|c|c|c|c|c|c|c|}
\hline & \multirow{2}{*}{$\begin{array}{l}\text { Operating } \\
\text { Condition }\end{array}$} & \multicolumn{5}{|c|}{ Without Catalyst } & \multicolumn{5}{|c|}{ With Catalyst } \\
\hline & & LPG & CNG & RFG & ETH & MTH & LPG & CNG & RFG & ETH & MTH \\
\hline \multirow[t]{3}{*}{ FTP } & Lean & 11,947 & 1,777 & 41,943 & 23,947 & 9,885 & 435 & 68 & 731 & 1,743 & 538 \\
\hline & Stoich. & 5,326 & 1,614 & 9,855 & 11,780 & 5,904 & 223 & 71 & 695 & 1,739 & 532 \\
\hline & Rich & 6,532 & 4,793 & 12,851 & 12,279 & 5,061 & 3,435 & 602 & 14,300 & 2,074 & 653 \\
\hline \multirow[t]{3}{*}{ Bag 2} & Lean & 13,960 & 2,125 & 54,206 & 29,916 & 11,113 & 125 & 47 & 93 & 91 & 29 \\
\hline & Stoich. & 5,942 & 1,894 & 10,710 & 10,969 & 5,385 & 30 & 32 & 11 & 24 & 24 \\
\hline & Bich & 7.104 & 5,497 & 14.377 & 10.577 & 4.320 & 3.487 & 533 & 16.259 & 19 & 30 \\
\hline
\end{tabular}




\section{Reactivity Adjustment Factors}

Average reactivity adjustment factors (RAFs) for each vehicle-fuel combination at all three operating conditions are presented in Table 16. RAFs were calculated as the ratio of the specific reactivities determined in these tests (g ozone/g NMOG) and the specific reactivity determined by CARB for a group of TLEV vehicles tested with RF-A fuel (3.42 $\mathrm{g}$ ozone/g NMOG). RAFs of post-catalyst exhaust during stoichiometric operation were $0.38,0.37,1.07$, 0.54, and 0.25 for LPG, CNG, RFG, ethanol, and methanol, respectively. At lean conditions, the reactivity of post-catalyst emissions was similar to stoichiometry. At fuel-rich conditions, the high concentrations of unburned fuel in the post-catalyst exhaust caused the RAFs for LPG and RFG to increase, whereas an increase in less reactive unburned fuel caused the reactivities of CNG, ethanol, and methanol to drop. The increase in RAF for LPG is most likely because of unburned propylene from the fuel and exhaust-formed ethylene passing through the catalyst. RAFs from LPG and CNG with the catalyst in place were about half the value of RAFs without the catalyst. This was due primarily to large reductions in ethylene, propylene, and formaldehyde concentrations across the catalyst. The catalyst appeared to have little influence on RAFs while operating on RFG. RAFs from ethanol and methanol with the catalyst in place were about $20 \%$ less than without the catalyst.

TABLE 16. REACTIVITY ADJUSTMENT FACTORS

\begin{tabular}{|c|c|c|c|c|c|c|c|c|c|c|}
\hline \multirow{2}{*}{$\begin{array}{l}\text { Operating } \\
\text { Condition }\end{array}$} & \multicolumn{5}{|c|}{ Without Catalyst } & \multicolumn{5}{|c|}{ With Catalyst } \\
\hline & LPG & CNG & RFG & ETH & MTH & LPG & CNG & RFG & ETH & MTH \\
\hline Lean & 0.71 & 1.12 & 1.08 & 0.65 & 0.33 & 0.38 & 0.37 & 1.07 & 0.54 & 0.25 \\
\hline Stoich. & 0.84 & 0.84 & 1.20 & 0.66 & 0.32 & 0.38 & 0.37 & 1.07 & 0.54 & 0.25 \\
\hline Rich & 0.76 & 0.75 & 1.03 & 0.62 & 0.31 & 0.56 & 0.31 & 1.16 & 0.52 & 0.24 \\
\hline
\end{tabular}

\section{E. Speciated Exhaust Emissions/Prevalent Species}

Prevalent exhaust species detected by GC-FID analysis are compiled in Appendices I through M. These species represent more than $95 \%$ of all measured NMOG mass detected by GC-FID at all operating conditions. The most prominent of these constituents observed for each fuel are listed in Table 17. These compounds account for more than $95 \%$ of all measured NMOG from the alternative fuels and more than $60 \%$ from reformulated gasoline. The predominant constituents of LPG and CNG exhaust were $\mathrm{C}_{1}-\mathrm{C}_{3}$ compounds. The most prevalent species in exhaust from reformulated gasoline were mostly $\mathrm{C}_{4}-\mathrm{C}_{8}$ compounds. Ethanol and methanol exhaust constituents were mostly $\mathrm{C}_{1}$ and $\mathrm{C}_{2}$ compounds. 
TABLE 17 - PROMINENT NON-METHANE EXHAUST SPECIES IDENTIFIED BY HYDROCARBON SPECIATION

\begin{tabular}{|c|c|c|c|c|c|}
\hline Fuel & LPG & CNG & RFG & Ethanol & Methanol \\
\hline $\begin{array}{l}\text { Prominent } \\
\text { Species }\end{array}$ & $\begin{array}{l}\text { ethane } \\
\text { ethylene } \\
\text { propane } \\
\text { propylene } \\
\text { acetylene } \\
\text { formaldehyde } \\
\text { acetaldehyde }\end{array}$ & $\begin{array}{l}\text { ethane } \\
\text { ethylene } \\
\text { propane } \\
\text { propylene } \\
\text { acetylene } \\
\text { formaldehyde } \\
\text { acetaldehyde }\end{array}$ & $\begin{array}{l}\text { ethylene } \\
\text { propylene } \\
\text { acetylene } \\
\text { isobutylene } \\
\text { isopentane } \\
\text { MTBE } \\
\text { benzene } \\
2,3-\text { dimethylpentane } \\
\text { 2,2,4-trimethylpentane } \\
\text { toluene } \\
\text { ethylbenzene } \\
\text { m- \& p-xylene } \\
\text { o-xylene } \\
\text { formaldehyde }\end{array}$ & $\begin{array}{l}\text { ethane } \\
\text { ethylene } \\
\text { acetylene } \\
\text { ethanol } \\
\text { formaldehyde } \\
\text { acetaldehyde }\end{array}$ & $\begin{array}{l}\text { ethylene } \\
\text { acetylene } \\
\text { methanol } \\
\text { toluene } \\
\text { formaldehyde }\end{array}$ \\
\hline NMOG wt\% & $>95 \%$ & $>95 \%$ & $>60 \%$ & $>97 \%$ & $>98 \%$ \\
\hline
\end{tabular}

\section{F. Mass Spectral Analyses}

In addition to the GC-FID analyses, GC/MS analyses were conducted on all exhaust samples. A number of compounds identified by GC-FID speciation were confirmed by GC/MS analysis. These compounds are listed in Table 18. Normally, hydrocarbons smaller than $\mathrm{C}_{6}$ were not adsorbed in the sorbent traps used in this study, and their identification by GC-FID speciation methods could not be confirmed by GC/MS analysis. Compounds that were not present at levels that could be detected by Auto/Oil speciation methods, but were identified by GC/MS analysis, are also listed in Table 18. Additional compounds identified by GC/MS analysis that were not identified by other analytical methods are listed in Table 19. Virtually all of the compounds identified were observed in exhaust sampled without a catalyst in place, or during fuel-rich operation on reformulated gasoline. Notable combustion products from all the fuels identified by GC/MS analysis included a number of nitrogen-containing compounds such as nitromethane, nitroethane, nitropropane, and nitropropane. In addition, a number of higher-molecular-weight compounds were observed in all exhaust samples. It is speculated that these compounds originated from the lubricating oil. 
TABLE 18. SPECIATED COMPOUNDS CONFIRMED BY GC-MS ANALYSIS

\begin{tabular}{|c|c|c|c|c|c|c|}
\hline \multirow{2}{*}{$\begin{array}{l}\text { Exhaust Configuration } \\
\text { FuelMir Equivalence Ratio }\end{array}$} & \multicolumn{3}{|c|}{ Without Catalyst } & \multicolumn{3}{|c|}{ With Catalyst } \\
\hline & 0.8 & 1.0 & 1.2 & 0.8 & 1.0 & 1.2 \\
\hline Methanol & $\begin{array}{c}\mathrm{LPG}^{\mathrm{b}} \\
\mathrm{CNG}^{\mathrm{b}} \\
\mathrm{RFG}^{\mathrm{b}} \\
\mathrm{MHOH}^{\mathrm{a}}\end{array}$ & $\begin{array}{l}\mathrm{LPG}^{\mathrm{b}} \\
\mathrm{CNG}^{\mathrm{b}} \\
\mathrm{RFG}^{\mathrm{b}} \\
\mathrm{MtOH}^{\mathrm{a}}\end{array}$ & $\begin{array}{l}\text { LPG }^{\mathrm{b}} \\
\mathrm{CNG}^{\mathrm{b}} \\
\mathrm{RFG}^{\mathrm{b}} \\
\mathrm{EtOH}^{\mathrm{c}} \\
\mathrm{MtOH}^{\mathrm{a}}\end{array}$ & $\begin{array}{l}\mathrm{LPG}^{\mathrm{b}} \\
\mathrm{CNG}^{\mathrm{b}} \\
\mathrm{M} \mathrm{OH}^{\mathrm{b}}\end{array}$ & $\begin{array}{l}\mathrm{LPG}^{\circ} \\
\mathrm{CNG}^{\mathrm{b}} \\
\mathrm{MtOH}^{\mathrm{a}}\end{array}$ & $\begin{array}{l}\text { LPG }^{b} \\
\mathrm{CNG}^{\mathrm{b}} \\
\mathrm{RFG}^{\mathrm{b}} \\
\mathrm{MHOH}^{\mathrm{b}}\end{array}$ \\
\hline Ethanol & $\begin{array}{l}\mathrm{EtOH}^{\mathrm{a}} \\
\mathrm{MtOH}^{\mathrm{c}}\end{array}$ & $\begin{array}{l}\mathrm{EtOH}^{\mathrm{a}} \\
\mathrm{MtOH}^{\mathrm{c}}\end{array}$ & $\mathrm{EtOH}^{2}$ & $\mathrm{EtOH}^{\mathrm{a}}$ & $\mathrm{EtOH}^{\mathrm{a}}$ & $\mathrm{EtOH}^{\mathrm{b}}$ \\
\hline Methylpropylbenzene & $\begin{array}{l}\mathrm{CNG}^{\mathrm{b}} \\
\mathrm{RFG}^{\mathrm{a}} \\
\mathrm{EtOH}^{\mathrm{b}}\end{array}$ & $\begin{array}{l}\mathrm{CNG}^{\mathrm{b}} \\
\mathrm{RFG}^{\mathrm{a}}\end{array}$ & $\begin{array}{l}\mathrm{CNG}^{\mathrm{b}} \\
\mathrm{RFG}^{\mathrm{a}} \\
\mathrm{EtOH}^{\mathrm{b}}\end{array}$ & $\mathrm{RFG}^{\mathrm{b}}$ & $\begin{array}{l}\mathrm{CNG}^{\mathrm{b}} \\
\mathrm{RFG}^{\mathrm{b}} \\
\mathrm{EtOH}^{\mathrm{b}}\end{array}$ & $\begin{array}{l}\mathrm{RFG}^{\mathrm{a}} \\
\mathrm{EtOH}^{\mathrm{b}}\end{array}$ \\
\hline Ethylbenzene & $\begin{array}{l}\mathrm{LPG}^{\mathrm{b}} \\
\mathrm{CNG}^{\mathrm{b}} \\
\mathrm{RFG}^{\mathrm{b}} \\
\mathrm{EtOH}\end{array}$ & $\begin{array}{l}\mathrm{LPG}^{\mathrm{b}} \\
\mathrm{CNG}^{\mathrm{b}} \\
\mathrm{RFG}^{\mathrm{b}} \\
\mathrm{EtOH}^{\mathrm{b}}\end{array}$ & $\begin{array}{l}\text { LPG }^{\mathrm{b}} \\
\mathrm{CNG}^{\mathrm{b}} \\
\mathrm{RFG}^{\mathrm{b}} \\
\mathrm{EtOH}^{\mathrm{b}}\end{array}$ & $\begin{array}{l}\mathrm{LPG}^{\mathrm{b}} \\
\mathrm{CNG}^{\mathrm{b}} \\
\mathrm{RFG}^{\mathrm{b}} \\
\mathrm{EtOH}^{\mathrm{b}}\end{array}$ & $\begin{array}{l}\mathrm{LPG}^{\mathrm{b}} \\
\mathrm{CNG}^{\mathrm{b}} \\
\mathrm{RFG}^{\mathrm{b}} \\
\mathrm{EtOH}^{\mathrm{b}}\end{array}$ & $\begin{array}{l}\mathrm{LPG}^{\mathrm{b}} \\
\mathrm{CNG}^{\mathrm{b}} \\
\mathrm{RFG}^{\mathrm{b}} \\
\mathrm{EtOH}^{\mathrm{b}}\end{array}$ \\
\hline Benzene & $\begin{array}{l}\mathrm{LPG}^{\mathrm{b}} \\
\mathrm{CNG}^{\mathrm{b}} \\
\mathrm{RFG}^{\mathrm{b}} \\
\mathrm{EtOH}^{\mathrm{a}} \\
\mathrm{MtOH}^{\mathrm{b}}\end{array}$ & $\begin{array}{l}\mathrm{LPG}^{\mathrm{a}} \\
\mathrm{CNG}^{\mathrm{b}} \\
\mathrm{RFG}^{\mathrm{b}} \\
\mathrm{EtOH}^{\mathrm{b}} \\
\mathrm{MtOH}^{\mathrm{b}}\end{array}$ & $\begin{array}{l}\mathrm{LPG}^{\mathrm{b}} \\
\mathrm{CNG}^{\mathrm{a}} \\
\mathrm{RFG}^{\mathrm{b}} \\
\mathrm{EtOH}^{\mathrm{b}} \\
\mathrm{MHOH}^{\mathrm{b}} \\
\end{array}$ & $\begin{array}{l}\mathrm{CNG}^{\mathrm{b}} \\
\mathrm{RFG}{ }^{\mathrm{b}} \\
\mathrm{EtOH}^{\mathrm{b}} \\
\mathrm{MtOH}^{\mathrm{b}}\end{array}$ & $\begin{array}{l}\mathrm{LPG}^{\mathrm{b}} \\
\mathrm{CNG}^{\mathrm{b}} \\
\mathrm{RFG}^{\mathrm{b}} \\
\mathrm{E} \mathrm{HOH}^{\mathrm{b}} \\
\mathrm{MtOH}^{\mathrm{a}}\end{array}$ & $\begin{array}{l}\mathrm{LPG}^{\mathrm{a}} \\
\mathrm{CNG}^{\mathrm{b}} \\
\mathrm{RFG}^{\mathrm{b}} \\
\mathrm{EtOH}^{\mathrm{b}} \\
\mathrm{MtOH} \\
\end{array}$ \\
\hline Diethylbenzene & $\begin{array}{l}\mathrm{CNG}^{\mathrm{b}} \\
\mathrm{RFG} \\
\mathrm{EtOH}^{\mathrm{c}}\end{array}$ & $\begin{array}{l}\mathrm{CNG}^{\mathrm{b}} \\
\mathrm{RFG}^{\mathrm{a}}\end{array}$ & $\begin{array}{l}\mathrm{LPG}^{\mathrm{b}} \\
\mathrm{RFG}^{\mathrm{a}}\end{array}$ & $\begin{array}{l}\mathrm{RFG}^{\mathrm{a}} \\
\mathrm{EtOH}^{\mathrm{c}}\end{array}$ & $\begin{array}{l}\mathrm{CNG}^{\mathrm{b}} \\
\mathrm{RFG}^{\mathrm{a}} \\
\mathrm{EtOH}^{\mathrm{c}}\end{array}$ & $\begin{array}{l}\mathrm{LPG}^{\mathrm{b}} \\
\mathrm{CNG}^{\mathrm{b}} \\
\mathrm{RFG}^{\mathrm{a}}\end{array}$ \\
\hline Dimethybenzene & $\begin{array}{l}\mathrm{CNG}^{\mathrm{b}} \\
\mathrm{RFG}^{\mathrm{a}}\end{array}$ & $\begin{array}{l}\mathrm{CNG}^{\mathrm{b}} \\
\mathrm{RFG}^{\mathrm{a}}\end{array}$ & $\mathrm{RFG}^{\mathrm{a}}$ & $\mathrm{RFG}^{\mathrm{b}}$ & $\mathrm{RFG}^{\mathrm{b}}$ & $\mathrm{RFG}^{\mathrm{a}}$ \\
\hline Methylethylbenzene & $\begin{array}{l}\mathrm{LPG}^{\mathrm{b}} \\
\mathrm{CNG}^{\mathrm{b}} \\
\mathrm{RFG} \\
\mathrm{EtOH}^{\mathrm{a}} \\
\mathrm{MtOH}^{\mathrm{c}}\end{array}$ & $\begin{array}{l}\mathrm{LPG}^{\mathrm{a}} \\
\mathrm{CNG}^{\mathrm{b}} \\
\mathrm{RFG}^{\mathrm{a}} \\
\mathrm{EtOH}^{\mathrm{b}}\end{array}$ & $\begin{array}{l}\mathrm{CNG}^{\mathrm{b}} \\
\mathrm{RFG} \\
\mathrm{EtOH}^{\mathrm{a}}\end{array}$ & $\begin{array}{l}\mathrm{LPG}^{\mathrm{b}} \\
\mathrm{CNG}^{\mathrm{b}} \\
\mathrm{RFG}^{\mathrm{b}} \\
\mathrm{EtOH}^{\mathrm{b}}\end{array}$ & $\begin{array}{l}\mathrm{CNG}^{\mathrm{b}} \\
\mathrm{RFG}^{\mathrm{b}} \\
\mathrm{EtOH}^{\mathrm{b}}\end{array}$ & $\begin{array}{l}\mathrm{CNG}^{\mathrm{b}} \\
\mathrm{RFG}^{\mathrm{a}} \\
\mathrm{EtOH}^{\mathrm{a}} \\
\mathrm{MtOH}^{\mathrm{a}}\end{array}$ \\
\hline Naphthalene & $R F G^{a}$ & $R F G^{a}$ & $\mathrm{RFG}^{\mathrm{a}}$ & $R F G^{c}$ & $\mathrm{RFG}^{\mathrm{c}}$ & $\begin{array}{l}\mathrm{RFG}^{\mathrm{a}} \\
\mathrm{EtOH}^{\mathrm{c}}\end{array}$ \\
\hline Propylbenzene & $\mathrm{RFG}^{\mathrm{a}}$ & $\mathrm{RFG}^{\mathrm{a}}$ & $\begin{array}{l}\mathrm{RFG}^{\mathrm{a}} \\
\mathrm{MtOH}^{\mathrm{c}}\end{array}$ & $\mathrm{RFG}^{\mathrm{b}}$ & $\begin{array}{c}\mathrm{CNG}^{\mathrm{b}} \\
\mathrm{RFG}^{\mathrm{b}} \\
\mathrm{MtOH}^{\mathrm{a}}\end{array}$ & $\begin{array}{l}\mathrm{RFG}^{\mathrm{a}} \\
\mathrm{EtOH}^{\mathrm{a}} \\
\mathrm{MtOH}^{\mathrm{c}}\end{array}$ \\
\hline Tetramethylbenzene & $\begin{array}{l}\mathrm{CNG}^{\mathrm{b}} \\
\mathrm{RFG}^{\mathrm{a}}\end{array}$ & $\mathrm{RFG}^{\mathrm{a}}$ & $\begin{array}{l}\mathrm{CNG}^{\mathrm{b}} \\
\mathrm{RFG}^{\mathrm{a}}\end{array}$ & $\begin{array}{l}\mathrm{LPG}^{\mathrm{b}} \\
\mathrm{CNG}^{\mathrm{b}} \\
\mathrm{RFG}^{\mathrm{a}}\end{array}$ & $\mathrm{RFG}^{\mathrm{a}}$ & $\mathrm{RFG}^{\mathrm{a}}$ \\
\hline
\end{tabular}


TABLE 18 (Cont'd). SPECIATED COMPOUNDS CONFIRMED BY GC-MS ANALYSIS

\begin{tabular}{|c|c|c|c|c|c|c|}
\hline \multirow{2}{*}{\begin{tabular}{|l} 
Exhaust Configuration \\
FuenAir Equivalence Ratio \\
\end{tabular}} & \multicolumn{3}{|c|}{ Without Catalyst } & \multicolumn{3}{|c|}{ With Catalyst } \\
\hline & 0.8 & 1.0 & 1.2 & 0.8 & 1.0 & 1.2 \\
\hline Trimethylbenzene & $\begin{array}{l}\mathrm{LPG}^{\mathrm{b}} \\
\mathrm{CNG}^{\mathrm{b}} \\
\mathrm{RFG}^{\mathrm{a}} \\
\mathrm{EtOH}^{\mathrm{a}} \\
\mathrm{MHOH}^{\mathrm{b}}\end{array}$ & $\begin{array}{l}\mathrm{LPG}^{\mathrm{a}} \\
\mathrm{CNG}^{\mathrm{b}} \\
\mathrm{RFG}^{\mathrm{a}} \\
\mathrm{EtOH}^{\mathrm{b}} \\
\mathrm{MtOH}^{\mathrm{b}}\end{array}$ & $\begin{array}{l}\mathrm{LPG}^{\mathrm{b}} \\
\mathrm{CNG}^{\mathrm{b}} \\
\mathrm{RFG}^{\mathrm{a}} \\
\mathrm{EtOH} \\
\mathrm{MtOH}^{\mathrm{a}}\end{array}$ & $\begin{array}{l}\mathrm{LPG}^{\mathrm{b}} \\
\mathrm{CNG}^{\mathrm{b}} \\
\mathrm{RFG} \\
\mathrm{EtOH} \\
\mathrm{MtOH}^{\mathrm{b}}\end{array}$ & $\begin{array}{l}\mathrm{LPG}^{\mathrm{b}} \\
\mathrm{CNG}^{\mathrm{b}} \\
\mathrm{RFG}^{\mathrm{a}} \\
\mathrm{EtOH}^{\mathrm{b}} \\
\mathrm{MHOH}^{\mathrm{a}}\end{array}$ & $\begin{array}{l}\mathrm{LPG}^{\mathrm{b}} \\
\mathrm{CNG}^{\mathrm{b}} \\
\mathrm{RFG}^{\mathrm{a}} \\
\mathrm{EtOH}^{\mathrm{a}} \\
\mathrm{MtOH}^{\mathrm{a}}\end{array}$ \\
\hline Methylpentane & $\begin{array}{l}\mathrm{LPG}^{\mathrm{b}} \\
\mathrm{CNG}^{\mathrm{b}} \\
\mathrm{RFG}^{\mathrm{a}} \\
\mathrm{EtOH}^{\mathrm{b}} \\
\mathrm{MHOH}^{\mathrm{a}}\end{array}$ & $\begin{array}{l}\text { LPG }^{\mathrm{b}} \\
\mathrm{CNG}^{\mathrm{b}} \\
\mathrm{RFG}^{\mathrm{a}} \\
\mathrm{EtOH}^{\mathrm{a}} \\
\mathrm{MtOH}^{\mathrm{b}}\end{array}$ & $\begin{array}{l}\mathrm{LPG}^{\mathrm{b}} \\
\mathrm{CNG}^{\mathrm{b}} \\
\mathrm{RFG}^{\mathrm{a}} \\
\mathrm{EtOH}^{\mathrm{b}} \\
\mathrm{MtOH}^{\mathrm{b}}\end{array}$ & $\begin{array}{l}\mathrm{LPG}^{\mathrm{b}} \\
\mathrm{CNG}^{\mathrm{b}} \\
\mathrm{RFG}^{\mathrm{a}} \\
\mathrm{EtOH}^{\mathrm{b}} \\
\mathrm{MHOH}^{\mathrm{a}}\end{array}$ & $\begin{array}{l}\mathrm{LPG}^{\mathrm{b}} \\
\mathrm{CNG}^{\mathrm{a}} \\
\mathrm{RFG}^{\mathrm{b}} \\
\mathrm{EtOH}^{\mathrm{b}} \\
\mathrm{MtOH}^{\mathrm{b}}\end{array}$ & $\begin{array}{l}\mathrm{LPG}^{\mathrm{b}} \\
\mathrm{CNG}^{\mathrm{b}} \\
\mathrm{RFG}^{\mathrm{a}} \\
\mathrm{EtOH}^{\mathrm{b}} \\
\mathrm{MtOH}^{\mathrm{a}}\end{array}$ \\
\hline Dimethylpentane & $\begin{array}{l}\mathrm{LPG}^{\mathrm{b}} \\
\mathrm{CNG}^{\mathrm{b}} \\
\mathrm{RFG}^{\mathrm{b}} \\
\mathrm{EtOH}^{\mathrm{b}} \\
\mathrm{MHOH}^{\mathrm{b}}\end{array}$ & $\begin{array}{l}\text { LPG }^{b} \\
\mathrm{CNG}^{\mathrm{b}} \\
\mathrm{RFG}^{\mathrm{b}} \\
\mathrm{EtOH}^{\mathrm{b}} \\
\mathrm{MtOH}^{\mathrm{b}}\end{array}$ & $\begin{array}{l}\mathrm{LPG}^{\mathrm{b}} \\
\mathrm{CNG}^{\mathrm{b}} \\
\mathrm{RFG}^{\mathrm{b}} \\
\mathrm{EtOH}^{\mathrm{b}} \\
\mathrm{M} \mathrm{HOH}{ }^{\mathrm{b}}\end{array}$ & $\begin{array}{l}\mathrm{LPG}^{\mathrm{b}} \\
\mathrm{CNG}^{\mathrm{b}} \\
\mathrm{RFG}^{\mathrm{b}} \\
\mathrm{EtOH}^{\mathrm{b}} \\
\mathrm{MHOH}^{\mathrm{b}}\end{array}$ & $\begin{array}{l}\text { LPG }^{b} \\
\mathrm{CNG}^{\mathrm{b}} \\
\mathrm{RFG}^{\mathrm{b}} \\
\mathrm{EtOH}^{\mathrm{b}} \\
\mathrm{MHOH}^{\mathrm{a}}\end{array}$ & $\begin{array}{l}\mathrm{LPG}^{\mathrm{b}} \\
\mathrm{CNG}^{\mathrm{b}} \\
\mathrm{RFG} \\
\mathrm{EtOH} \\
\mathrm{MtOH}^{\mathrm{b}}\end{array}$ \\
\hline Dimethylbutene & $R F G^{b}$ & $\begin{array}{l}\mathrm{RFG}^{\mathrm{b}} \\
\mathrm{EtOH}^{\mathrm{c}}\end{array}$ & $\begin{array}{c}\mathrm{RFG}^{\mathrm{b}} \\
{\mathrm{M}+\mathrm{OH}^{\mathrm{b}}}^{\mathrm{s}}\end{array}$ & & & $R F G^{b}$ \\
\hline Methylcyclopentane & $\begin{array}{l}\text { LPG }^{\mathrm{b}} \\
\mathrm{RFG}^{\mathrm{b}}\end{array}$ & $\begin{array}{l}\mathrm{LPG}^{\mathrm{b}} \\
\mathrm{CNG}^{\mathrm{b}} \\
\mathrm{RFG}^{\mathrm{b}} \\
\end{array}$ & $\begin{array}{l}\text { LPG }^{b} \\
\text { RFG }^{b}\end{array}$ & $\begin{array}{l}\mathrm{LPG}^{\mathrm{b}} \\
\mathrm{CNG}^{\mathrm{b}} \\
\mathrm{RFG}^{\mathrm{b}}\end{array}$ & $\begin{array}{l}\mathrm{RFG}^{\mathrm{b}} \\
\mathrm{EtOH}^{\mathrm{c}}\end{array}$ & $\mathrm{RFG}^{\mathrm{b}}$ \\
\hline Propylcyclohexane & & & & & & $\mathrm{MtOH}^{\mathrm{c}}$ \\
\hline Dodecane & $\begin{array}{l}\mathrm{CNG}^{\mathrm{b}} \\
\mathrm{RFG}^{\mathrm{b}}\end{array}$ & $\begin{array}{l}\mathrm{LPG}^{\mathrm{b}} \\
\mathrm{CNG}^{\mathrm{a}} \\
\mathrm{RFG}^{\mathrm{b}}\end{array}$ & $\begin{array}{l}\mathrm{LPG}^{\mathrm{b}} \\
\mathrm{CNG}^{\mathrm{b}} \\
\mathrm{RFG}^{\mathrm{b}}\end{array}$ & $\begin{array}{l}\mathrm{CNG}^{\mathrm{b}} \\
\mathrm{RFG}^{\mathrm{b}}\end{array}$ & $\begin{array}{l}\mathrm{LPG}^{\mathrm{b}} \\
\mathrm{CNG}^{\mathrm{b}} \\
\mathrm{RFG}^{\mathrm{b}}\end{array}$ & $\begin{array}{l}\mathrm{CNG}^{\mathrm{b}} \\
\mathrm{RFG}^{\mathrm{b}} \\
\mathrm{EtOH}^{\mathrm{b}}\end{array}$ \\
\hline Heptane & $\begin{array}{l}\mathrm{LPG}^{\mathrm{b}} \\
\mathrm{CNG}^{\mathrm{b}} \\
\mathrm{RFG}^{\mathrm{a}} \\
\mathrm{EtOH}^{\mathrm{a}} \\
\mathrm{MHOH}^{\mathrm{b}}\end{array}$ & $\begin{array}{l}\mathrm{LPG}^{\mathrm{b}} \\
\mathrm{CNG}^{\mathrm{b}} \\
\mathrm{RFG}^{\mathrm{a}} \\
\mathrm{EtOH}^{\mathrm{a}} \\
\mathrm{MHOH}^{\mathrm{b}}\end{array}$ & $\begin{array}{l}\mathrm{LPG}^{\mathrm{b}} \\
\mathrm{CNG}^{\mathrm{b}} \\
\mathrm{RFG}^{\mathrm{a}} \\
\mathrm{E} \mathrm{HOH}^{\mathrm{b}} \\
\mathrm{M} \mathrm{HOH}^{\mathrm{b}}\end{array}$ & $\begin{array}{l}\mathrm{LPG}^{\mathrm{b}} \\
\mathrm{CNG}^{\mathrm{b}} \\
\mathrm{RFG}^{\mathrm{b}} \\
\mathrm{EtOH}^{\mathrm{b}} \\
\mathrm{MtOH}^{\mathrm{b}}\end{array}$ & $\begin{array}{l}\mathrm{LPG}^{\mathrm{b}} \\
\mathrm{CNG}^{\mathrm{b}} \\
\mathrm{RFG}{ }^{\mathrm{b}} \\
\mathrm{EtOH} \mathrm{H}^{\mathrm{b}} \\
\mathrm{MtOH}^{\mathrm{b}}\end{array}$ & $\begin{array}{l}\mathrm{LPG}^{\mathrm{b}} \\
\mathrm{CNG}^{\mathrm{b}} \\
\mathrm{RFG}^{\mathrm{b}} \\
\mathrm{EtOH}^{\mathrm{a}} \\
\mathrm{MtOH}^{\mathrm{a}}\end{array}$ \\
\hline 1-Heptene & $\begin{array}{l}\mathrm{EtOH}^{\mathrm{c}} \\
\mathrm{M}^{\mathrm{O} O \mathrm{H}^{\mathrm{c}}} \\
\end{array}$ & & & & & \\
\hline Hexane & $\begin{array}{l}\mathrm{LPG}^{\mathrm{b}} \\
\mathrm{CNG}^{\mathrm{b}} \\
\mathrm{RFG}^{\mathrm{a}} \\
\mathrm{EtOH} \\
\mathrm{MtOH}^{\mathrm{a}}\end{array}$ & $\begin{array}{l}\mathrm{LPG}^{\mathrm{b}} \\
\mathrm{CNG}^{\mathrm{b}} \\
\mathrm{RFG}^{\mathrm{a}} \\
\mathrm{EtOH}^{\mathrm{a}} \\
\mathrm{MtOH}^{\mathrm{b}}\end{array}$ & $\begin{array}{l}\mathrm{LPG}^{\mathrm{b}} \\
\mathrm{CNG}^{\mathrm{b}} \\
\mathrm{RFG}^{\mathrm{a}} \\
\mathrm{EtOH}^{\mathrm{b}} \\
\mathrm{MHOH}^{\mathrm{b}}\end{array}$ & $\begin{array}{l}\mathrm{LPG}^{\mathrm{b}} \\
\mathrm{CNG}^{\mathrm{b}} \\
\mathrm{RFG}^{\mathrm{a}} \\
\mathrm{EtOH}^{\mathrm{a}} \\
\mathrm{MHOH}^{\mathrm{a}}\end{array}$ & $\begin{array}{l}\mathrm{LPG}^{\mathrm{b}} \\
\mathrm{CNG}^{\mathrm{b}} \\
\mathrm{RFG}^{\mathrm{a}} \\
\mathrm{EtOH}^{\mathrm{a}} \\
\mathrm{MtOH}^{\mathrm{b}}\end{array}$ & $\begin{array}{l}\mathrm{LPG}^{\mathrm{b}} \\
\mathrm{CNG}^{\mathrm{b}} \\
\mathrm{RFG}^{\mathrm{a}} \\
\mathrm{EtOH}^{\mathrm{b}} \\
\mathrm{MHOH}^{\mathrm{b}}\end{array}$ \\
\hline Methylpropane & $\begin{array}{l}\mathrm{LPG}^{\mathrm{b}} \\
\mathrm{CNG}^{\mathrm{b}} \\
\mathrm{RFG}^{\mathrm{b}} \\
\mathrm{EtOH}^{\mathrm{b}}\end{array}$ & $\begin{array}{l}\mathrm{LPG}^{\mathrm{b}} \\
\mathrm{CNG}^{\mathrm{b}} \\
\mathrm{RFG}^{\mathrm{b}} \\
\mathrm{EtOH}^{\mathrm{b}} \\
\mathrm{M} \mathrm{OH} \mathrm{H}^{\mathrm{b}}\end{array}$ & $\begin{array}{l}\mathrm{LPG}^{\mathrm{a}} \\
\mathrm{CNG}^{\mathrm{b}} \\
\mathrm{RFG}^{\mathrm{b}} \\
\mathrm{MHOH}^{\mathrm{b}}\end{array}$ & $\begin{array}{l}\mathrm{LPG}^{\mathrm{b}} \\
\mathrm{CNG}^{\mathrm{b}} \\
\mathrm{RFG}^{\mathrm{b}} \\
\mathrm{EtOH}^{\mathrm{b}}\end{array}$ & $\begin{array}{l}\mathrm{LPG}^{\mathrm{b}} \\
\mathrm{CNG}^{\mathrm{b}} \\
\mathrm{RFG}^{\mathrm{b}} \\
\mathrm{EtOH}^{\mathrm{b}}\end{array}$ & $\begin{array}{l}\mathrm{LPG}^{\mathrm{b}} \\
\mathrm{CNG}^{\mathrm{b}} \\
\mathrm{RFG} \\
\mathrm{E} \mathrm{HOH}^{\mathrm{b}} \\
\mathrm{M} \mathrm{\textrm {O } O H}\end{array}$ \\
\hline
\end{tabular}


TABLE 18 (Cont'd). SPECIATED COMPOUNDS CONFIRMED BY GC-MS ANALYSIS

\begin{tabular}{|c|c|c|c|c|c|c|}
\hline \multirow{2}{*}{$\begin{array}{l}\text { Exhaust Configuration } \\
\text { FuelMir Equivalence Ratio }\end{array}$} & \multicolumn{3}{|c|}{ Without Catalyst } & \multicolumn{3}{|c|}{ With Catalyst } \\
\hline & 0.8 & 1.0 & 1.2 & 0.8 & 1.0 & 1.2 \\
\hline Methylheptane & $\begin{array}{l}\mathrm{LPG}^{\mathrm{b}} \\
\mathrm{RFG}^{\mathrm{a}}\end{array}$ & $\begin{array}{l}\mathrm{LPG}^{\mathrm{b}} \\
\mathrm{CNG}^{\mathrm{b}} \\
\mathrm{RFG}^{\mathrm{a}}\end{array}$ & $\begin{array}{c}\mathrm{LPG}^{\mathrm{b}} \\
\mathrm{CNG}^{\mathrm{b}} \\
\mathrm{RFG}^{\mathrm{a}} \\
\mathrm{MtOH}^{\mathrm{b}}\end{array}$ & $\begin{array}{l}\mathrm{LPG}^{\circ} \\
\mathrm{RFG}^{\mathrm{b}} \\
\mathrm{EtOH}^{\mathrm{a}} \\
\mathrm{MtOH}{ }^{\circ}\end{array}$ & $\begin{array}{l}\mathrm{LPG}^{\mathrm{b}} \\
\mathrm{CNG}^{\mathrm{b}} \\
\mathrm{RFG}^{\mathrm{b}}\end{array}$ & $\begin{array}{l}\mathrm{LPG}^{b} \\
\mathrm{CNG}^{\mathrm{b}} \\
\mathrm{RFG}^{\mathrm{b}} \\
\mathrm{EtOH}^{\mathrm{b}}\end{array}$ \\
\hline Methylhexane & $\begin{array}{l}\mathrm{LPG}^{\mathrm{b}} \\
\mathrm{CNG}^{\mathrm{b}} \\
\mathrm{RFG}^{\mathrm{a}} \\
\mathrm{EtOH}^{\mathrm{a}} \\
\mathrm{MtOH}^{\mathrm{b}}\end{array}$ & $\begin{array}{l}\text { LPG }^{b} \\
\mathrm{CNG}^{\mathrm{b}} \\
\mathrm{RFG}^{\mathrm{a}} \\
\mathrm{EtOH}^{\mathrm{a}} \\
\mathrm{MtOH}^{\mathrm{b}}\end{array}$ & $\begin{array}{l}\mathrm{LPG}^{\mathrm{b}} \\
\mathrm{CNG}^{\mathrm{b}} \\
\mathrm{RFG}^{\mathrm{a}} \\
\mathrm{EtOH}^{\mathrm{b}} \\
\mathrm{MtOH}^{\mathrm{b}}\end{array}$ & $\begin{array}{l}\mathrm{LPG}^{\mathrm{b}} \\
\mathrm{CNG}^{\mathrm{b}} \\
\mathrm{RFG}^{\mathrm{a}} \\
\mathrm{EtOH}^{\mathrm{b}} \\
\mathrm{MtOH}^{\mathrm{b}}\end{array}$ & $\begin{array}{l}\mathrm{LPG}^{\mathrm{b}} \\
\mathrm{CNG}^{\mathrm{b}} \\
\mathrm{RFG}^{\mathrm{a}} \\
\mathrm{EtOH} \\
\mathrm{MtOH}^{\mathrm{b}}\end{array}$ & $\begin{array}{l}\mathrm{LPG}^{\mathrm{b}} \\
\mathrm{CNG}^{\mathrm{b}} \\
\mathrm{RFG}^{\mathrm{b}} \\
\mathrm{EtOH}^{\mathrm{b}} \\
\mathrm{MHOH}^{\mathrm{c}}\end{array}$ \\
\hline Methylpentene & $\begin{array}{l}\mathrm{LPG}^{\mathrm{b}} \\
\mathrm{CNG}^{\mathrm{b}} \\
\mathrm{RFG}^{\mathrm{a}} \\
\mathrm{EtOH}^{\mathrm{b}} \\
\mathrm{MtOH}^{\mathrm{b}}\end{array}$ & $\begin{array}{l}\mathrm{LPG}^{\mathrm{b}} \\
\mathrm{CNG}^{\mathrm{b}} \\
\mathrm{RFG}^{\mathrm{a}} \\
\mathrm{EtOH}^{\mathrm{b}} \\
\mathrm{MtOH}^{\mathrm{b}}\end{array}$ & $\begin{array}{l}\mathrm{LPG}^{\mathrm{b}} \\
\mathrm{CNG}^{\mathrm{b}} \\
\mathrm{RFG}^{\mathrm{a}} \\
\mathrm{EtOH}^{\mathrm{b}} \\
\mathrm{MtOH}^{\mathrm{b}}\end{array}$ & $\begin{array}{l}\mathrm{LPG}^{\mathrm{b}} \\
\mathrm{CNG}^{\mathrm{b}} \\
\mathrm{RFG}^{\mathrm{b}} \\
\mathrm{EtOH}^{\mathrm{b}} \\
\mathrm{MtOH}^{\mathrm{b}}\end{array}$ & $\begin{array}{l}\mathrm{LPG}^{\mathrm{b}} \\
\mathrm{CNG}^{\mathrm{b}} \\
\mathrm{RFG}^{\mathrm{b}} \\
\mathrm{EtOH}^{\mathrm{b}} \\
\mathrm{MtOH}^{\mathrm{a}}\end{array}$ & $\begin{array}{l}\mathrm{LPG}^{\mathrm{b}} \\
\mathrm{CNG}^{\mathrm{b}} \\
\mathrm{RFG}^{\mathrm{b}} \\
\mathrm{EtOH}^{\mathrm{b}} \\
\mathrm{MHOH}^{\mathrm{b}}\end{array}$ \\
\hline Octane & $\mathrm{RFG}^{\mathrm{b}}$ & $\begin{array}{l}\mathrm{RFG}^{\mathrm{b}} \\
\mathrm{EtOH}^{\mathrm{a}}\end{array}$ & $\begin{array}{l}\mathrm{RFG}^{\mathrm{b}} \\
\mathrm{MtOH}^{\mathrm{b}}\end{array}$ & $\mathrm{RFG}^{\mathrm{b}}$ & $\begin{array}{l}\mathrm{LPG}^{\mathrm{b}} \\
\mathrm{CNG}^{\mathrm{b}} \\
\mathrm{RFG}^{\mathrm{b}}\end{array}$ & $\begin{array}{l}\mathrm{LPG}^{\mathrm{b}} \\
\mathrm{CNG}^{\mathrm{b}} \\
\mathrm{RFG}^{\mathrm{b}} \\
\mathrm{EtOH}^{\mathrm{b}}\end{array}$ \\
\hline Methyloctane & $\begin{array}{l}\mathrm{RFG}^{b} \\
\mathrm{EtOH}^{c}\end{array}$ & $\begin{array}{l}\mathrm{CNG}^{\mathrm{b}} \\
\mathrm{RFG}^{\mathrm{b}} \\
\mathrm{EtOH}^{\mathrm{b}}\end{array}$ & $\mathrm{RFG}^{\mathrm{b}}$ & $R F G^{b}$ & $\begin{array}{l}\mathrm{LPG}^{\mathrm{b}} \\
\mathrm{RF} \mathrm{G}^{\mathrm{b}}\end{array}$ & $\begin{array}{l}\mathrm{RFG}^{\mathrm{b}} \\
\mathrm{EtOH}^{\mathrm{b}}\end{array}$ \\
\hline Dimethyloctane & $\begin{array}{l}\mathrm{LPG}^{\mathrm{b}} \\
\mathrm{CNG}^{\mathrm{b}} \\
\mathrm{RFG}{ }^{\mathrm{b}}\end{array}$ & $\begin{array}{l}\mathrm{LPG}^{\mathrm{b}} \\
\mathrm{CNG}^{\mathrm{b}} \\
\mathrm{RFG}^{\mathrm{b}}\end{array}$ & $\begin{array}{c}\mathrm{LPG}^{\mathrm{b}} \\
\mathrm{CNG}^{\mathrm{b}} \\
\mathrm{RFG}^{\mathrm{b}} \\
\mathrm{MtOH}^{\mathrm{b}}\end{array}$ & $\begin{array}{l}\mathrm{CNG}^{\mathrm{b}} \\
\mathrm{RFG}^{\mathrm{b}} \\
\mathrm{EtOH}^{\mathrm{b}}\end{array}$ & $\begin{array}{l}\mathrm{LPG}^{\mathrm{b}} \\
\mathrm{CNG}^{\mathrm{b}} \\
\mathrm{RFG}^{\mathrm{b}}\end{array}$ & $\begin{array}{l}\mathrm{LPG}^{\mathrm{b}} \\
\mathrm{CNG}^{\mathrm{b}} \\
\mathrm{RFG}^{\mathrm{b}} \\
\mathrm{EtOH}^{\mathrm{b}} \\
\mathrm{MtOH}^{\mathrm{C}}\end{array}$ \\
\hline Undecane & $\begin{array}{l}\mathrm{CNG}^{\mathrm{b}} \\
\mathrm{RFG}^{\mathrm{b}} \\
\mathrm{MtOH}^{\mathrm{b}}\end{array}$ & $\begin{array}{l}\mathrm{LPG}^{\mathrm{b}} \\
\mathrm{CNG}^{\mathrm{a}} \\
\mathrm{RFG}^{\mathrm{b}} \\
\mathrm{MtOH}^{\mathrm{b}}\end{array}$ & $\begin{array}{c}\mathrm{LPG}^{\mathrm{b}} \\
\mathrm{CNG}^{\mathrm{b}} \\
\mathrm{RFG}^{\mathrm{b}} \\
\mathrm{MTOH}^{\mathrm{b}}\end{array}$ & $\begin{array}{l}\mathrm{CNG} \\
\mathrm{RFG}^{\mathrm{b}} \\
\mathrm{E} \mathrm{CH}^{\mathrm{b}}\end{array}$ & $\begin{array}{c}\mathrm{LPG}^{\mathrm{b}} \\
\mathrm{CNG}^{\mathrm{a}} \\
\mathrm{RFG}^{\mathrm{b}} \\
\mathrm{MtOH}^{\mathrm{b}}\end{array}$ & $\begin{array}{l}\mathrm{LPG}^{\mathrm{b}} \\
\mathrm{CNG}^{\mathrm{b}} \\
\mathrm{RFG}^{\mathrm{b}} \\
\mathrm{EtOH}^{\mathrm{b}}\end{array}$ \\
\hline Methhylethylketone & $\mathrm{RFG}^{\mathrm{a}}$ & $\mathrm{RFG}^{\mathrm{a}}$ & $\begin{array}{l}\mathrm{RFG}^{\mathrm{a}} \\
\mathrm{EtOH}^{\mathrm{b}}\end{array}$ & $\begin{array}{l}\mathrm{RFG}^{\mathrm{a}} \\
\mathrm{EtOH}^{\mathrm{b}} \\
\mathrm{MtOH}^{\mathrm{b}}\end{array}$ & $\begin{array}{c}\mathrm{RFG}^{\mathrm{a}} \\
\mathrm{EtOH}^{\mathrm{b}} \\
\mathrm{MtOH}^{\mathrm{b}}\end{array}$ & $\begin{array}{l}\mathrm{RFG}^{\mathrm{a}} \\
\mathrm{EtOH}^{\mathrm{b}} \\
\mathrm{MtOH}^{\mathrm{b}}\end{array}$ \\
\hline Isobutyraldehyde & $\begin{array}{l}\mathrm{LPG}^{a} \\
\mathrm{CNG}^{\mathrm{b}} \\
\mathrm{RFG}^{\mathrm{b}}\end{array}$ & $R F G^{b}$ & $\begin{array}{l}\mathrm{LPG}^{\mathrm{b}} \\
\mathrm{RFG}^{\mathrm{b}} \\
\mathrm{EtOH}\end{array}$ & $\begin{array}{c}\mathrm{RFG}^{\mathrm{b}} \\
\mathrm{EtOH}^{\mathrm{b}} \\
\mathrm{MtOH}^{\mathrm{b}}\end{array}$ & $\begin{array}{l}\mathrm{LPG}^{\mathrm{b}} \\
\mathrm{RFG}^{\mathrm{b}} \\
\mathrm{EtOH}^{\mathrm{b}} \\
\mathrm{MHOH}^{\mathrm{b}}\end{array}$ & $\begin{array}{l}\mathrm{LPG}^{\mathrm{b}} \\
\mathrm{RFG}^{\mathrm{b}} \\
\mathrm{EtOH}^{\mathrm{D}} \\
\mathrm{MtOH}^{\mathrm{b}}\end{array}$ \\
\hline
\end{tabular}


TABLE 19. OTHER COMPOUNDS IDENTIFIED BY GC-MS ANALYSIS

\begin{tabular}{|c|c|c|c|c|c|c|}
\hline \multirow{2}{*}{\begin{tabular}{|l} 
Exhaust Configuration \\
Fuel/Air Equivalence Ratio
\end{tabular}} & \multicolumn{3}{|c|}{ Without Catalyst } & \multicolumn{3}{|c|}{ With Catalyst } \\
\hline & 0.8 & 1.0 & 1.2 & 0.8 & 1.0 & 1.2 \\
\hline \multicolumn{7}{|c|}{ AROMATICS } \\
\hline 1,1(1,2-ethanediyl)bis-4-methyl-benzene & RFG & RFG & RFG & & & RFG \\
\hline 1,2,3,4-tetrahydro-methyl-naphthalene & RFG & RFG & RFG & & & RFG \\
\hline 1-Ethyl-2,3-dihydro-1-methyl-1H-indene & RFG & RFG & RFG & & & RFG \\
\hline acenaphthylene & RFG & RFG & RFG & & & RFG \\
\hline azulene & $\mathrm{EtOH}$ & & & $\mathrm{EtOH}$ & & \\
\hline biphenylene & & $\mathrm{EtOH}$ & & & & \\
\hline cis-decahydro-naphthalene & & LPG & & & & \\
\hline cyclodecane & & & & & & $\mathrm{EtOH}$ \\
\hline cyclohexadiene & & & & & & $\mathrm{EtOH}$ \\
\hline Ethyl-dimethylethyl-benzene & RFG & RFG & RFG & & & RFG \\
\hline Ethyl-naphthalene & RFG & RFG & RFG & & & RFG \\
\hline isopropyl-cyclobutane & & EtOH & & & & \\
\hline methyl-(1-methylethyl)-benzene & RFG & RFG & RFG & & & RFG \\
\hline methyl-naphthalene & RFG & $\begin{array}{l}\text { CNG } \\
\text { RFG }\end{array}$ & $\begin{array}{l}\text { CNG } \\
\text { RFG } \\
\text { EtOH } \\
\end{array}$ & & RFG & RFG \\
\hline methylethyl-naphthalene & RFG & RFG & RFG & & & RFG \\
\hline phenanthrene & $\mathrm{RFG}$ & RFG & RFG & & & RFG \\
\hline 1,1'-(1,2-dimethyl-1,2-ethanediyl)bis-benzene & $\mathrm{RFG}$ & RFG & RFG & & & RFG \\
\hline \multicolumn{7}{|c|}{ AROMATIC - UNSATURATED } \\
\hline 1, 1'-(1,2-ethanediyl)bis-benzene & $\mathrm{RFG}$ & RFG & RFG & & & $\mathrm{RFG}$ \\
\hline $1,1^{\prime}$-(1-methyl-1,2-ethanediyl)bis-benzene & RFG & RFG & RFG & & & RFG \\
\hline ethynyl-benzene & LPG & LPG & LPG & & & \\
\hline ethynyl-ethyl-benzene & RFG & RFG & RFG & RFG & RFG & RFG \\
\hline \multicolumn{7}{|c|}{ AROMATIC - OXYGEN-CONTAINING } \\
\hline 1-(4-ethylphenyl)-ehtanone & & & & & & $\mathrm{EtOH}$ \\
\hline 1-methyl-4-methylene-cyclohexane & & & & & & $\mathrm{EHOH}$ \\
\hline 1-napthalene-carboxaldehyde & & & & & & RFG \\
\hline 1-phenyl-ethanone & & & $\mathrm{MtOH}$ & & CNG & \\
\hline 2-naphthyl-aldehyde & RFG & RFG & & & & RFG \\
\hline 2,5-biphenyl-phenol & & CNG & & & & \\
\hline 3-ethyl-phenol & & & & & & $\mathrm{EtOH}$ \\
\hline benzene-acetaldehyde & RFG & RFG & & & & RFG \\
\hline benzoic acid & $\begin{array}{l}\text { LPG } \\
\text { EtOH } \\
\text { M+OH }\end{array}$ & MtOH & $\mathrm{MtOH}$ & LPG & & \\
\hline
\end{tabular}


TABLE 19 (Cont'd). OTHER COMPOUNDS IDENTIFIED BY GC-MS ANALYSIS

\begin{tabular}{|c|c|c|c|c|c|c|}
\hline \multirow{2}{*}{\begin{tabular}{|l|} 
Exhaust Configuration \\
FueVAir Equivalence Ratio \\
\end{tabular}} & \multicolumn{3}{|c|}{ Without Catalyst } & \multicolumn{3}{|c|}{ With Catalyst } \\
\hline & 0.8 & 1.0 & 1.2 & 0.8 & 1.0 & 1.2 \\
\hline diethyl-benzyl-ethanol & $\mathrm{RFG}$ & RFG & RFG & & & RFG \\
\hline dimethyl-benzaldehyde & RFG & RFG & RFG & & & RFG \\
\hline ethyl-benzaldehyde & RFG & RFG & RFG & & & RFG \\
\hline ethylmethyl-phenol & & & & & & $\mathrm{EtOH}$ \\
\hline methyl-benzaldehyde & RFG & RFG & RFG & & & $\begin{array}{r}\text { RFG } \\
\text { MtOH } \\
\end{array}$ \\
\hline methyl-phenol & RFG & RFG & RFG & & & $\begin{array}{l}\mathrm{RFG} \\
\mathrm{MtOH}\end{array}$ \\
\hline methyl-pentanone & & & & EtOH & & $\mathrm{EtOH}$ \\
\hline p-(2-methylallyl)-phenol & RFG & RFG & RFG & & & RFG \\
\hline phenyl-maleic anhydride & $\begin{array}{l}\mathrm{EtOH} \\
\mathrm{MtOH} \\
\end{array}$ & CNG & CNG & & & \\
\hline toluene-methanol & RFG & RFG & RFG & & & RFG \\
\hline trimethyl-2-cyclopentane-1-one & & & & & & EtOH \\
\hline \multicolumn{7}{|c|}{ OXYGEN-CONTAINING } \\
\hline 1-butanol, 3-methyl, formate & $\mathrm{MtOH}$ & & & & & \\
\hline 1-methylethylester & & & & & LPG & \\
\hline |-hydroxy-4,5-diethyl-2(1HO-pyridinethione) & $\begin{array}{l}\mathrm{EtOH} \\
\mathrm{MtOH}\end{array}$ & & & $\begin{array}{l}\mathrm{EtOH} \\
\mathrm{MtOH}\end{array}$ & & \\
\hline |2,5-dihydrofuran & RFG & RFG & RFG & & & $\begin{array}{l}\text { RFG } \\
\text { EtOH }\end{array}$ \\
\hline 2-butanone & RFG & RFG & RFG & & & RFG \\
\hline 2-ethyl-1-butanol & RFG & RFG & RFG & & & RFG \\
\hline 2-butanoic acid & & & & & & $\mathrm{EtOH}$ \\
\hline |2-methoxy-2-methyl-propane & RFG & RFG & $\begin{array}{l}\text { RFG } \\
\text { EtOH } \\
\end{array}$ & & & RFG \\
\hline 2-methyl-2-propanol & $\mathrm{MtOH}$ & & & & & \\
\hline 6 carbon ketone & RFG & $\mathrm{RFG}$ & RFG & & & \\
\hline acetic acid ethyl ester & EtOH & $\mathrm{EtOH}$ & EtOH & & & \\
\hline |.alpha.-methyl-benzene-methanol & EtOH & $\mathrm{EtOH}$ & EtOH & & & EtOH \\
\hline |1-(1-propenyl)-bicyclo[3.2.1]octane-2-one & RFG & $\mathrm{RFG}$ & RFG & & & \\
\hline butanediol & RFG & RFG & RFG & & & RFG \\
\hline butenol & & & & EtOH & EtOH & EtOH \\
\hline diethoxy-ethane & & & & EtOH & $\mathrm{EtOH}$ & \\
\hline |ethyl-oxirane & & $\mathrm{MtOH}$ & & & & \\
\hline formic acid ethyl ester & $\mathrm{MtOH}$ & & EtOH & & & \\
\hline hexanoicacid & $\mathrm{FtOH}$ & & $\mathrm{FHOH}$ & & & \\
\hline
\end{tabular}


TABLE 19 (Cont'd). OTHER COMPOUNDS IDENTIFIED BY GC-MS ANALYSIS

\begin{tabular}{|c|c|c|c|c|c|c|}
\hline \multirow{2}{*}{$\begin{array}{l}\text { Exhaust Configuration } \\
\text { Fuel/Air Equivalence Ratio }\end{array}$} & \multicolumn{3}{|c|}{ Without Catalyst } & \multicolumn{3}{|c|}{ With Catalyst } \\
\hline & 0.8 & 1.0 & 1.2 & 0.8 & 1.0 & 1.2 \\
\hline isopropyl-myristate & $\begin{array}{l}\text { EtOH } \\
\mathrm{MtOH}\end{array}$ & $\begin{array}{l}\text { EtOH } \\
\mathrm{MtOH}\end{array}$ & MtOH & & EtOH & \\
\hline methyl-butanol & & & & & & $\mathrm{EtOH}$ \\
\hline methyl-pentadiene & & & & & & EtOH \\
\hline phenyl-methanol & RFG & RFG & RFG & & & RFG \\
\hline propanol & $\mathrm{MHOH}$ & & $\mathrm{EtOH}$ & & EtOH & $\mathrm{MtOH}$ \\
\hline tetradecanoic acid & & CNG & & & LPG & \\
\hline \multicolumn{7}{|c|}{ OXYGEN-CONTAINING - UNSATURATED } \\
\hline 2-phenyl-2-butenal & & RFG & RFG & & & \\
\hline 3-heptene-2-ol & & & & & & EtOH \\
\hline 3-pentene-2-one & & & & & & EtOH \\
\hline 4-hexene-1-ol & & & & & & EtOH \\
\hline diethyl-ketene & RFG & RFG & RFG & & & \\
\hline \multicolumn{7}{|c|}{ UNSATURATED } \\
\hline 1,3,7-octatrien-5-yne & $\mathrm{RFG}$ & $\mathrm{RFG}$ & $\mathrm{RFG}$ & & & \\
\hline 3,4-nonadiene & & & & EtOH & & \\
\hline 3-methyl-decane & & & CNG & & & \\
\hline cycloheptatriene & $\mathrm{EtOH}$ & & & & & \\
\hline dimethyl-decene & $\mathrm{MtOH}$ & & & & & \\
\hline dimehtyl-heptene & EtOH & & & & & \\
\hline hexadiyne & $\begin{array}{l}\text { RFG } \\
\text { EtOH } \\
\text { MtOH }\end{array}$ & $\begin{array}{l}\text { RFG } \\
\text { EtOH }\end{array}$ & $\begin{array}{l}\text { RFG } \\
\text { EtOH }\end{array}$ & & & \\
\hline \multicolumn{7}{|c|}{ SATURATED } \\
\hline 2-methyl-butane & & & $\mathrm{MtOH}$ & & & \\
\hline$C_{15}$ branched alkane & & & & & & $\mathrm{EtOH}$ \\
\hline$C_{17}$ branched alkane & $\begin{array}{l}\text { RFG } \\
\text { MtOH }\end{array}$ & $\begin{array}{l}\text { RFG } \\
\text { MtOH }\end{array}$ & $\begin{array}{l}\text { RFG } \\
\text { EtOH } \\
\text { MtOH } \\
\end{array}$ & $\mathrm{MtOH}$ & $\mathrm{MtOH}$ & $\begin{array}{l}\text { RFG } \\
\text { EtOH } \\
\mathrm{MHOH} \\
\end{array}$ \\
\hline $\mathrm{C}_{18}$ branched alkane & $\mathrm{MtOH}$ & & & & & EtOH \\
\hline$C_{19}$ branched alkane & $\mathrm{MtOH}$ & $\mathrm{MtOH}$ & $\begin{array}{l}\text { EtOH } \\
\mathrm{MtOH}\end{array}$ & MtOH & & $\begin{array}{l}\text { EtOH } \\
\mathrm{MHOH}\end{array}$ \\
\hline $\mathrm{C}_{20}$ branched alkane & $\mathrm{MtOH}$ & $\mathrm{MtOH}$ & $\begin{array}{l}\mathrm{EtOH} \\
\mathrm{MtOH}\end{array}$ & $\mathrm{MtOH}$ & & $\begin{array}{l}\mathrm{EtOH} \\
\mathrm{MHOH}\end{array}$ \\
\hline dimethyloxy-dimethyl-cyclohexane & & & & & $\mathrm{MtOH}$ & \\
\hline eicosane & & $\mathrm{MtOH}$ & & & $\mathrm{MtOH}$ & EtOH \\
\hline
\end{tabular}


TABLE 19 (Cont'd). OTHER COMPOUNDS IDENTIFIED BY GC-MS ANALYSIS

\begin{tabular}{|c|c|c|c|c|c|c|}
\hline \multirow{2}{*}{\begin{tabular}{|l|} 
Exhaust Configuration \\
FueVAir Equivalence Ratio \\
\end{tabular}} & \multicolumn{3}{|c|}{ Without Catalyst } & \multicolumn{3}{|c|}{ With Catalyst } \\
\hline & 0.8 & 1.0 & 1.2 & 0.8 & 1.0 & 1.2 \\
\hline hexadecane & $\mathrm{MtOH}$ & $\mathrm{MtOH}$ & $\begin{array}{l}\mathrm{EHOH} \\
\mathrm{MHOH}\end{array}$ & $\mathrm{MtOH}$ & $\overline{\mathrm{MtOH}}$ & $\overline{\mathrm{EtOH}}$ \\
\hline methyl-tetradecane & RFG & RFG & RFG & & & RFG \\
\hline nonadecane & $\mathrm{MtOH}$ & $\mathrm{MtOH}$ & $\begin{array}{l}\mathrm{EtOH} \\
\mathrm{MHOH}\end{array}$ & $\mathrm{MtOH}$ & $\mathrm{MtOH}$ & $\begin{array}{l}\mathrm{EtOH} \\
\mathrm{MtOH} \\
\end{array}$ \\
\hline octadecane & $\mathrm{MtOH}$ & $\mathrm{MtOH}$ & $\begin{array}{l}\text { EtOH } \\
\text { MtOH }\end{array}$ & $\mathrm{MtOH}$ & MtOH & $\begin{array}{l}\mathrm{EtOH} \\
\mathrm{MtOH} \\
\end{array}$ \\
\hline pentadecane & MtOH & $\begin{array}{l}\mathrm{CNG} \\
\mathrm{M}+\mathrm{OH}\end{array}$ & $\begin{array}{l}\text { EtOH } \\
\text { MOOH }\end{array}$ & $\mathrm{MtOH}$ & $\mathrm{MtOH}$ & $\begin{array}{l}\text { EtOH } \\
\text { MtOH }\end{array}$ \\
\hline tetradecane & $\mathrm{MtOH}$ & $\mathrm{CNG}$ & EtOH & & $\mathrm{MtOH}$ & \\
\hline tetramethyl-cyclopropane & EtOH & EtOH & & & & \\
\hline tetramethyl-hexadecane & & & & & $\mathrm{MtOH}$ & \\
\hline trimethyl-dodecane & RFG & RFG & RFG & & & RFG \\
\hline \multicolumn{7}{|c|}{ NITROGEN-CONTAINING } \\
\hline 1-cyclopropyl-4-nitro-benzene & RFG & RFG & & & & \\
\hline 1-nitropropane & LPG & LPG & & & & \\
\hline 1-nitro-2-propanol & & LPG & & & & \\
\hline 2,4-dimethyl-2-nitro-pentane & RFG & RFG & RFG & & & \\
\hline 2-nitropropane & LPG & LPG & LPG & & & \\
\hline nitroethane & LPG & $\begin{array}{l}\text { LPG } \\
\text { EtOH }\end{array}$ & $\begin{array}{l}\text { LPG } \\
\mathrm{CNG} \\
\mathrm{EtOH}\end{array}$ & & & \\
\hline nitromethane & \begin{tabular}{|l} 
LPG \\
EtOH \\
MtOH
\end{tabular} & $\begin{array}{l}\text { EtOH } \\
\text { MtOH }\end{array}$ & $\begin{array}{l}\mathrm{CNG} \\
\mathrm{EtOH}\end{array}$ & & & \\
\hline \multicolumn{7}{|c|}{ SULFUR-CONTAINING } \\
\hline sulfonylbis-methane & & $\mathrm{MtOH}$ & & EtOH & $\begin{array}{l}\text { EtOH } \\
\text { MtOH } \\
\end{array}$ & $\mathrm{MtOH}$ \\
\hline sulfur dioxide & & EtOH & & & & \\
\hline
\end{tabular}




\section{SUMMARY AND CONCLUSIONS}

Regulated and volatile organic exhaust species were characterized from a 1993 Chevrolet Lumina operating on compressed natural gas (CNG), liquefied petroleum gas (LPG), and reformulated gasoline (RFG), and from a 1988 Chevrolet Corsica operating on ethanol (EtOH) and methanol (MtOH). For the evaluation of gaseous fuels, aftermarket conversion kits were installed on the Lumina. The Corsica was a dedicated alcohol vehicle owned by the University of Tennessee. For all fuels, the vehicles were operated over the chassis dynamometer portion of the Federal Test Procedure (FTP) for light-duty vehicles at fuel/air equivalence ratios of $0.8,1.0$, and 1.2 ; exhaust emissions were sampled both with and without the catalytic converter in place. The objective of the program was to qualitatively identify organic emissions from alternative-fueled vehicles during normal operation and simulated failure modes. To provide additional information for the program, these vehicle exhaust emissions were also quantified. Vehicles were tuned on each fuel to provide adequate driveability over the FTP; optimization of the vehicles to provide the lowest emissions levels was outside the scope of this program.

Analyses of exhaust samples included determination of regulated exhaust emissions by Code of Federal Regulations methods, hydrocarbon speciation, analyses of aldehydes and ketones according to Auto/Oil Phase II methods, and the determination of trace exhaust species by mass spectral analysis methods. In addition, a limited comparison of the ozoneforming potential of each vehicle/fuel combination was conducted based on the Maximum Incremental Reactivity scale as used by the California Air Resources Board for determining reactivity of individual exhaust species. Some of the findings in the study are listed below.

- Speciation data showed greater than $95 \%$ of all LPG and CNG organic exhaust constituents to be $\mathrm{C}_{1}-\mathrm{C}_{3}$ compounds.

- Prevalent species in exhaust from reformulated gasoline were mostly $\mathrm{C}_{4}-\mathrm{C}_{8}$ compounds.

- For the alcohol fuels, more than $96 \%$ of organic exhaust species were $\mathrm{C}_{1}$ and $\mathrm{C}_{2}$ compounds.

- More than $99 \%$ of measured NMOG mass could be attributed to 34 species in CNG exhaust, 54 species in LPG exhaust, more than 200 species in reformulated gasoline exhaust, 7 species in ethanol exhaust, and 5 species in methanol exhaust.

- Because of poor vehicle driveability on neat alcohols, unburned fuel accounted for virtually all NMOG emissions during operation on ethanol and methanol.

- Acetaldehyde accounted for virtually all toxic emissions while operating on ethanol, while formaldehyde composed practically all toxic emissions while operating on methanol. 
- On average, Reactivity Adjustment Factors (RAFs) of CNG and LPG exhaust were about $65 \%$ less than those of reformulated gasoline, whereas RAFs from ethanol and methanol were $50 \%$ and $75 \%$ less, respectively, than those of reformulated gasoline.

- Potential ozone produced by post-catalyst CNG and LPG emissions was less than half of that produced by RFG during stoichiometric operation. The alcohol fuels had RAFs of a level similar to the gaseous fuels at those operating conditions; however, high NMOG mass emissions rates resulted in ozone forming potentials for ethanol and methanol exhaust that were similar to reformulated gasoline.

- GC/MS analysis identified a number of nitrogen-containing compounds in exhaust samples from all fuels, including nitromethane, nitroethane, and nitropropane. A number of heavier compounds, likely from the lubricating oil, were also identified in exhaust from all fuels.

\section{RECOMMENDATIONS}

The primary objective of this program was to identify organic compounds qualitatively in the exhaust of vehicles operating at various conditions on alternative fuels. Identification efforts were successful and the basic project goal was met. In addition, exhaust emissions were quantified in an attempt to provide fuel-to-fuel comparisons of exhaust emissions. However, these comparisons were difficult due to the variability of the vehicles used to generate exhaust. None of the vehicle-fuel combinations evaluated in this program were optimized to provide the lowest exhaust emissions. Both gaseous conversion kits operated rich at idle under certain conditions. In addition, the alcohol-fueled vehicle ran rough and drove poorly when it was not fully warmed up. For all alternative fuels tested, especially the alcohol fuels, insufficient fuel system calibrations affected the level of exhaust emissions. This was particularly apparent in the cold-start portion of the FTP. A possible means of lessening the influence of the poor vehicle calibrations would be to examine and compare the Bag 2 exhaust emissions generated in this program. Some Bag 2 observations are noted in this report; however, additional effort in this area may be warranted.

In future studies examining exhaust emissions levels, OEM alternative-fueled vehicles or the latest generation of electronically-controlled fuel-injected gaseous conversion kits should be used when possible. This would preclude examining M100 and E100, as OEM alcohol vehicles are currently limited to a maximum of M85 and E85. However, a current TLEVcertified flexible-fueled vehicle fitted with the appropriate electronically-controlled gaseous fuel conversion kit should provide a more direct comparison of exhaust emissions levels from alternative fuels.

The extreme fuel/air equivalence ratios examined during this program were representative of severe fuel-control failure modes; however, in almost all instances vehicle driveability was severely degraded. It is likely that such vehicle failures would be quickly repaired. Of more concern are vehicles operating slightly rich or slightly lean. These conditions may not be noticeable to the driver, but may severely effect the performance of a 
catalytic converter. It is suggested that these types of in-use situations are more likely to occur and go unnoticed for long periods of time, and it may require additional work to quantify their effects.

The technique used to sample trace species for mass spectral analysis worked well for $\mathrm{C}_{6}$ and higher species; however, a quantification of a number of these species was not possible. Additional refinements to the method are needed to provide emission rates for these compounds. The presence of a number of nitrogen-containing organic compounds (e.g., nitromethane, nitroethane, nitropropane) in the exhaust from all four alternative fuels is noteworthy, and additional analytical efforts are needed to investigate the presence of these compounds.

\section{REFERENCES}

1. Code of Federal Regulations, Title 40, Part 86, Subpart B.

2. King, S., "Natural Gas as a Stationary Engine and Vehicular Fuel," SAE Paper 912364, 1991.

3. Painter, L. and Rutherford, J., "Statistical Design and Analysis Methods for the Auto/Oil Quality Research Program, SAE Paper 920319, 1992.

4. Bass, E.A., Evaluation of Aftermarket LPG Conversion Kits in Light-Duty Vehicle Applications, NREL TP-421-5462, Golden, CO: National Renewable Energy Laboratory, June 1993.

5. California Code of Regulations, Title 13, Section 1960.1, "California Exhaust Emission Standards and Test Procedures for 1988 and Subsequent Model Passenger Cars, LightDuty Trucks, and Medium-Duty Vehicles," amended June 24, 1996. 


\section{APPENDIX A}

COMPUTER PRINTOUT OF EMISSIONS DATA FROM "CHECK-OUT" FTP OF CHEVROLET LUMINA 
SOUTHWEST RESEARCH INSTITUTE - DEPARTMENYT OF EMISSIONS RESEARCE

COMPUTER PROGRAM LDT $1.5-R$

VEHICLE NUMBER 53M

VEHICLE MODEL 93 CHEVY LUMINA

ENGINE

3.1 I (189 CID) $-\mathrm{V}-6$

I4

5693 MILES ( $9160 \mathrm{KM}$ )

\section{3-BAG EPA FTP VEHICLE EMISSION RESULTS}

BAROMETER 29.42 IN HG $(747.3 \mathrm{MM} \mathrm{HG})$ RELATIVE HUMIDITY 58.6 PCT.

BAG NUMBER

BAG DESCRIPTION

RUN TIME SECONDS

DRY/WET CORRECTION FACTOR, SAMP/BACK

MEASURED DISTANCE MILES (KM)

BLOWER FLOW RATE SCFM (SCMM)

GAS METER FLOW RATE SCFM (SCMM)

TOTAL FLOW SCF (SCM)

HC SAMPLE METER/RANGE/PPM (BAG)

HC BCKGRD METER/RANGE/PPM

CO SAMPLE METER/RANGE/PPM

CO BCKGRD METER/RANGE/PPM

CO2 SAMPLE METER/RANGE/PCT

$\mathrm{CO} 2$ BCKGRD METER/RANGE/PCT

NOX SAMPLE METER/RANGE/PPM

NOX BCKGRD METER/RANGE/PRM

(BAG) (D)

DILUTION FACTOR

HC CONCENTRATION PPM

CO CONCENTRATION PPM

$\mathrm{CO} 2$ CONCENTRATION PCT

NOX CONCENTRATION PPM

HC MASS GRAMS

CO MASS GRAMS

$\mathrm{CO} 2$ MASS GRAMS

NOX MASS GRAMS

FUEL MASS KG

FUEL ECONOMY MPG (L/100KM)

3-BAG COMPOSITE RESULTS

$\begin{array}{llr}\text { HC } & \text { G/MI } & .314 \\ \text { CO } & \text { G/MI } & 6.021 \\ \text { NOX } & \text { G/MI } & .593\end{array}$

FUEL ECONOMY MPG (L/100KM)

$18.71(12.57)$

$\begin{array}{rrr}13.4 \prime & 1 / & 3.35 \\ 1.0 / & 1 / & .25\end{array}$

FUEL ECONOMY MPG (L/10OKM) 18.71 (12.57)

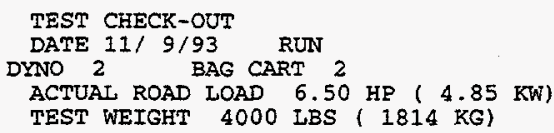

DRY BULB TEMPERATURE $75.0^{\circ} \mathrm{F}\left(23.9^{\circ} \mathrm{C}\right)$

PROTECT NO 08-6068-001

GASOLINE AS RECD

FUEL DENSITY $6.160 \mathrm{LB} / \mathrm{GAL}$ H $.134 \mathrm{C} .8660 .000 \mathrm{X} .000$

NOX HUMIDITY C.F. 1.012

$$
\begin{gathered}
1 \\
\text { COLD TRANSIENT } \\
(0-505 \text { SEC. }) \\
505.7 \\
.975 / .982 \\
3.65(5.87) \\
561.8(15.91) \\
.00(1.00) \\
4735 .(134.1)
\end{gathered}
$$

$49.812 / \quad 49.77$

$6.1 / 21 \quad 6.10$

$56.2 / 14 / 254.13$

$\begin{array}{lll}.2 / & 14 / & .81\end{array}$

$13.8 / 14 / .0470$

$\begin{array}{rrr}13.8 / & 14 / & 0470 \\ 72.9 / & 1 / & 18.23\end{array}$

1.01

$$
\begin{gathered}
2 \\
\text { STABILIZED } \\
(505-1372 \text { SEC. }) \\
867.8 \\
.978 / .982 \\
3.91(6.29) \\
563.0(15.95) \\
.00(2.00) \\
8143 .(230.6)
\end{gathered}
$$

$\begin{array}{lll}90.2 / & 1 / & 9.05\end{array}$

$59.6 / \quad 1 / \quad 5.98$

$43.2 / 12 / 42.04$

$1.5 / 12 / 1.43$

$70.3 / 14 / \quad .5037$

$13.8 / 14 / .0470$

17.73

44.02

245.00

6868

18.00

3.403

38.251

1686.17

4. 570

$18.42(12.77)$

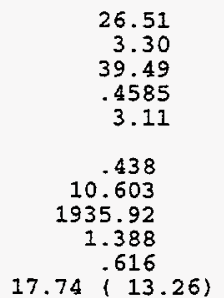

3

HOT TRANSIENT

(0) 505 SEC.

505.2

$.976 / .982$

$3.65(5.88)$

$563.5(15.96)$

$00(.00)$

4745. ( 134.4$)$

$16.2 / 2 / \quad 16.19$

$5.8 / 2 / \quad 5.80$

$88.1 / 13 / 215.15$

$.5 / 13 / 1.09$

$13.9 / 14 / 0474$

$\begin{array}{rrr}30.5 / & 1 / & 7.63 \\ 1.1 / & 1 / & .28\end{array}$

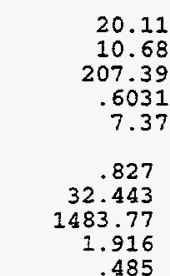

$21.06(11.17)$ 


\section{APPENDIX B}

COMPUTER PRINTOUTS OF EMISSIONS DATA FROM REFORMULATED GASOLINE BASELINE FTPS OF CHEVROLET LUMINA 
SOUTHWEST RESEARCH INSTITUTE - DEPARTMENT OF EMISSIONS RESEARCH

COMPUTER PROGRAM IDT $1.5-\mathrm{R}$
3-BAG EPA FTP VEHICLE EMISSION RESULTS

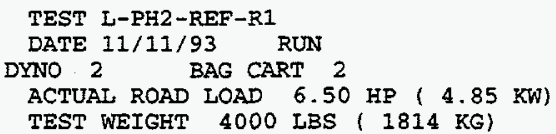

BAROMETER 29.21 IN HG $(741.9 \mathrm{MM} \mathrm{HG})$ RELATIVE HUMIDITY 56.0 PCT.

BAG NUMBER

BAG DESCRIPTION

RUN TIME SECONDS

DRY/WET CORRECTION FACTOR, SAMP/BACK

MEASURED DISTANCE MILES (KM)

BLOWER FLOW RATE SCFM (SCMM)

GAS METER FLOW RATE SCFM (SCMM)

TOTAL FLOW SCF (SCM)

HC SAMPLE METER/RANGE/PPM (BAG)

HC BCKGRD METER/RANGE/PPM

CO SAMPIE METER/RANGE/PPM

CO BCKGRD METER/RANGE/PPM

CO2 SAMPLE METER/RANGE/PCT

CO2 BCKGRD METER/RANGE/PCT

NOX SAMPLE METER/RANGE/PPM

NOX BCKGRD METER/RANGE/PRM

(BAG) (D)

DILUTION FACTOR

HC CONCENTRATION PPM

CO CONCENTRATION PPM

$\mathrm{CO} 2$ CONCENTRATION PCT

NOX CONCENTRATION PPM

HC MASS GRAMS

CO MASS GRAMS

$\mathrm{CO} 2$ MASS GRAMS

NOX MASS GRAMS

FUEL MASS KG

FUEL ECONOMY MPG (I/100KM)

(BAG)
(BAG) (D)

DRY BULB TEMPERATURE

$77.0^{\circ} \mathrm{F}\left(25.0^{\circ} \mathrm{C}\right)$

$$
\begin{gathered}
1 \\
\text { COLD TRANSIENT } \\
(0-505 \text { SEC. }) \\
504.9 \\
.975 / .982 \\
3.64(5.86) \\
564.4(15.98) \\
.00(1.00) \\
4750 .(134.5)
\end{gathered}
$$

$42.1 / 2 / 42.08$

$8.9 / 2 / \quad 8.89$

$80.2 / 13 / 193.48$

$.9 / 13 / 1.96$

$84.4 / 14 / \quad .7432$

$13.5 / 14 / \quad .0458$

$49.4 / \quad 1 / 12.36$

$2.7 / \quad 1 / \quad .68$

17.38

33.69

185.34

$$
.7001
$$$$
11.72
$$

2. 671

29.022

1723.97

3.086

.573

$17.75(13.26)$

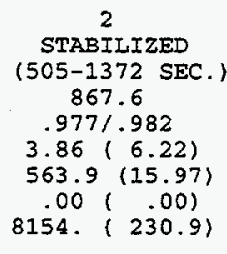

$10.8 / 2 / \quad 10.79$

$9.8 / 2 / \quad 9.79$

$19.3 / 12 / 18.61$

2.01121190

$70.4 / 14 / .5051$

$13.4 / 14 / .0454$

$5.6 / 1 / 1.40$

$.7 / 1 / \quad .18$

26.22

1.37

16.29

.4615

187

4.380

1951.07

.557

.631

$17.09\left(\begin{array}{l}.631 \\ (13.76)\end{array}\right.$

PROJECT NO. 08-6068-001

GASOLINE EM-1611-F

FUEL DENSITY 6.157 IB/GAL

H .137 C . $.8470 .016 \quad \mathrm{X} .000$

NOX HUMIDITY C.F. 1.024

HOT TRANSIENT

( $0-505$ SEC.)

505.2

$.975 / .982$

$3.62(5.82)$

$563.4\langle 15.95\rangle$

$.00\{.00\}$

4744. (134.4)

$14.5 / 2 / \quad 14.49$

$8.9 / 2 / \quad 8.89$

$42.0 / 13 / 95.82$

$.5 / 13 / 1.09$

$80.6 / 14 / .6698$

$13.0 / 14 / .0438$

$23.3 / 1 / 5.83$

$.7 / 1 / \quad .18$

3-BAG COMPOSITE RESULTS

$\begin{array}{llr}\text { HC } & \text { G/MI } & .214 \\ \text { CO } & \text { G/MI } & 3.342 \\ \text { NOX } & \text { G/MI } & .365\end{array}$

FUEL ECONOMY MPG (L/100KM)

$17.95(13.10)$

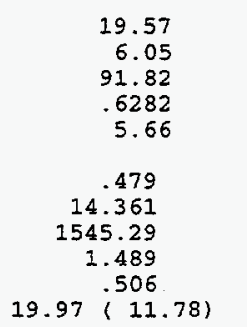


COMPUTER PROGRAM LDT $1.5-R$

VEHICIE NUMBER 53M

VEHICLE MODEL 93 CHEVY LUMINA

ENGINE

3.1 I (189 CID) $-\mathrm{V}-6$

TRANSMISSION $L 4$

ODOMETER $\quad 5743$ MILES ( $9240 \mathrm{KM})$

BAROMETER 28.98 IN HG (736.1 MM HG) RELATIVE HUMIDITY 57.2 PCT.

BAG NUMBER

BAG DESCRIPTION

RUN TIME SECONDS

DRY/WET CORRECTION FACTOR, SAMP/BACK

MEASURED DISTANCE MILES (KM)

BLOWER FLOW RATE SCFM (SCMM)

GAS METER FLOW RATE SCFM (SCMM)

TOTAL FLOW SCF (SCM)

$\begin{array}{lll}\text { HC } & \text { SAMPLE METER/RANGE/PPM (BAG) } \\ \text { HC } & \text { BCKGRD METER/RANGE/PPM } & \\ \text { CO SAMPLE METER/RANGE/PPM } & \\ \text { CO } & \text { BCKGRD METER/RANGE/PPM } & \\ \text { CO2 } & \text { SAMPLE METER/RANGE/PCT } & \\ \text { CO2 } & \text { BCKGRD METER/RANGE/PCT } & \\ \text { NOX SAMPLE METER/RANGE/PPM (BAG) } & \text { (D) } \\ \text { NOX } & \text { BCKGRD METER/RANGE/PPM } & \end{array}$

NOX BCKGRD METER/RANGE/PPM

DILUTION FACTOR

HC CONCENTRATION PPM

CO CONCENTRATION REM

$\mathrm{CO} 2$ CONCENTRATION PCT

NOX CONCENTRATION PPM

HC MASS GRAMS

CO MASS GRAMS

$\mathrm{CO} 2$ MASS GRAMS

NOX MASS GRAMS

FUEL MASS KG

FUEL ECONOMY MPG (L/100KM)

3-BAG COMPOSITE RESULTS

$\begin{array}{llr}\text { HC } & \text { G/MI } & .253 \\ \text { CO } & \text { G/MI } & 4.637 \\ \text { NOX } & \text { G/MI } & .323 \\ \text { FUEL } & \text { ECONOMY MPG } & \text { (L/100KM }\end{array}$

$17.95(13.10)$

FUEL ECONOMY MPG (L/100KM)
3-BAG ERA FTP VEHICLE EMISSION RESULTS

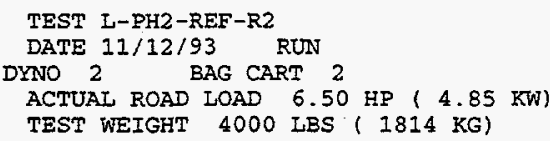

DRY BULS TEMPERATURE $79.0^{\circ} \mathrm{F}\left(26.1^{\circ} \mathrm{C}\right)$

$$
\begin{gathered}
1 \\
\text { COLD TRANSIENT } \\
(0-505 \text { SEC. }) \\
505.2 \\
.973 / .980 \\
3.61(5.80) \\
555.0(15.72) \\
.00(1.00) \\
4673 .(132.3)
\end{gathered}
$$

$44.2 / 2 / 44.17$

$6.9 / 2 / 6.90$

$53.9 / 14 / 242.10$

$.2 / \quad 14 / \quad .81$

$84.7 / 14 / \quad .7493$

$14.8 / 14 / .0510$

$\begin{array}{rrr}47.9 / & 1 / & 11.98 \\ .7 / & 1 / & .18\end{array}$

$$
\begin{gathered}
17.14 \\
37.68 \\
233.32 \\
.7013 \\
11.82 \\
2.939 \\
35.948 \\
1699.10 \\
3.183 \\
.569 \\
17.71(13.28)
\end{gathered}
$$

STABILIZED

(505-1372 SEC.) 867.2

$.975 / .980$

$3.86(6.20)$

$557.4(15.79)$

$00(.00)$

8056 . (228.2)

$76.5 / 1 / 7.68$

$55.3 / 1 / 6.55$

$18.3 / 12 / 17.63$

$1.2 / 12 / 1.14$

$71.2 / 14 / .5166$

$14.6 / 14 / .0502$

$5.4 / \quad 1 / \quad 1.35$

25.66

1. 38

16.05

.4683

1.23

.185

4.264

1956.30

.571

.633

$17.02(13.82)$
PROJECT NO. 08-6068-001

GASOLINE EM-1611-F

FUEI DENSITY 6.157 IB/GAL

H. $.137 \quad \mathrm{C} .847 \quad 0.016 \quad \mathrm{X} .000$

NOX HUMIDITY C.F. 1.065

HOT TRANSIENT

( $0-505$ SEC.)

505.2

$.974 / .980$

$3.62(5.83)$

$557.0(15.77)$

$00(.00\}$

4690. (132.8)

$$
\begin{array}{rrr}
16.9 / & 2 / & 16.89 \\
7.4 / & 2 / & 7.40 \\
73.7 / & 13 / & 176.03 \\
.6 / & 13 / & 1.31 \\
80.6 / & 14 / & .6698 \\
14.7 / & 14 / & .0506 \\
12.7 / & 1 / & 3.18 \\
.5 / & 1 / & .13 \\
& 19.34 \\
9.88 \\
169.25 \\
.6218 \\
3.06 \\
.773 \\
26.171 \\
1512.03 \\
.827 \\
20.17
\end{array}
$$




\section{APPENDIX C}

\section{COMPUTER PRINTOUTS OF EMISSIONS DATA FROM FTPs WITH LPG}

\begin{tabular}{|c|l|l|l||}
\hline \hline Page C- & \multicolumn{1}{|c|}{ Test Number } & $\begin{array}{l}\text { Operating } \\
\text { Condition }\end{array}$ & \multicolumn{1}{|c|}{$\begin{array}{c}\text { Catalyst } \\
\text { Installation }\end{array}$} \\
\hline \hline 1 & L-LPG-0.8-E1 & Lean & Without Catalyst \\
\hline 2 & L-LPG-0.8-E2 & Lean & Without Catalyst \\
\hline 3 & L-LPG-0.8-C1 & Lean & With Catalyst \\
\hline 4 & L-LPG-0.8-C2 & Lean & With Catalyst \\
\hline \hline 5 & L-LPG-1.0-E1 & Stoich & Without Catalyst \\
\hline 6 & L-LPG-1.0-E2 & Stoich & Without Catalyst \\
\hline 7 & L-LPG-1.0-C1 & Stoich & With Catalyst \\
\hline 8 & L-LPG-1.0-C2 & Stoich & With Catalyst \\
\hline \hline 9 & L-LPG-1.2-E1 & Rich & Without Catalyst \\
\hline 10 & L-LPG-1.2-E2 & Rich & Without Catalyst \\
\hline 11 & L-LPG-1.2-C1 & Rich & With Catalyst \\
\hline 12 & L-LPG-1.2-C2 & Rich & With Catalyst \\
\hline \hline
\end{tabular}


COMPUTER PROGRAM LDT $1.5-R$

VEHICLE NUMBER 53M

VEHICLE MODEL 93 CHEVY LUMINA

ENGINE

3.1 I (189 CID) $-\mathrm{V}-6$

14

TRANSMISSION

ODOMETER 6120 MILES ( $9847 \mathrm{KM}$ )

BAROMETER 29.64 IN HG (752.9 MM HG)

RELATIVE HUMIDITY 21.6 PCT.

BAG NUMBET

BAG DESCRIPTION

RUN TIME SECONDS

DRY/WET CORRECTION FACTOR, SAMP/BACK

MEASURED DISTANCE MILES (KM)

BLOWER FLOW RATE SCFM (SCMM)

GAS METER FLOW RATE SCFM (SCMM)

TOTAL FLOW SCF (SCM)

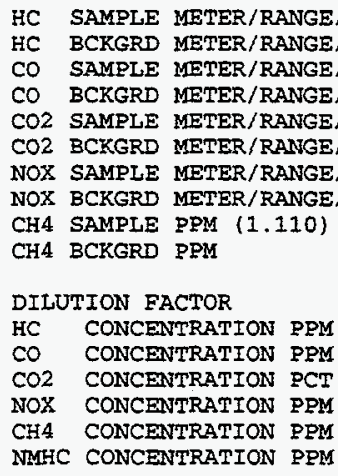

THC MASS GRAMS

CO MASS GRAMS

$\mathrm{CO} 2$ MASS GRAMS

NOX MASS GRAMS

$\mathrm{CH} 4$ MASS GRAMS

NMHC MASS GRAMS (FID)

FUEL MASS KG

FUEL ECONOMY MPG (L/100KM)

\section{(BAG)}

$\begin{array}{llll}14.1 / & 14 / & .0482\end{array}$

\begin{abstract}
3-BAG CARB FTP VEHICLE EMISSION RESULTS
\end{abstract}

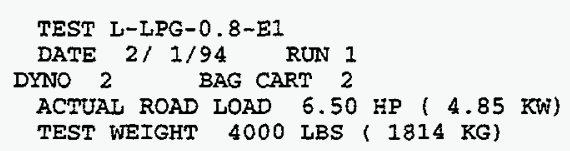

DRY BULB TEMPERATURE $72.0^{\circ} \mathrm{F}\left(22.2^{\circ} \mathrm{C}\right)$

$$
\begin{gathered}
1 \\
\text { COLD TRANSIENT } \\
(0-505 \text { SEC. }) \\
505.0 \\
.986 / .994 \\
3.64(5.86) \\
569.3(16.12) \\
.28(.01) \\
4794 .(135.8)
\end{gathered}
$$$$
\begin{array}{rrr}
19.2 / & 3 / & 191.57 \\
.8 / & 3 / & 7.98 \\
54.1 / & 13 / & 125.52 \\
.2 / & 13 / & .44 \\
79.7 / & 14 / & .6535 \\
14.1 / & 14 / & .0482 \\
30.5 / & 2 / & 30.51 \\
.5 / & 2 / & .50 \\
& 4.76 & \\
& 2.54
\end{array}
$$$$
17.07
$$$$
\begin{array}{r}
17.07 \\
184.06
\end{array}
$$$$
122.34
$$$$
.6081
$$$$
30.04
$$$$
2.36
$$$$
181.43
$$

15.555

19.336

1511.60

6.325
.214

14.204

.530

$17.90(13.14)$

$$
\begin{gathered}
2 \\
\text { STABILIZED } \\
(505-1372 \text { SEC. }) \\
867.6 \\
.989 / .994 \\
3.88(6.25) \\
572.3(16.21) \\
.29(.01) \\
8280 .(234.5)
\end{gathered}
$$

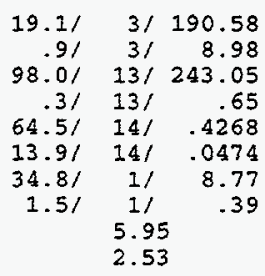

$\begin{array}{rrr}19.1 / & 3 / & 190.58 \\ .9 / & 3 / & 8.98 \\ 98.0 / & 13 / & 243.05 \\ .3 / & 13 / & .65 \\ 54.5 / & 14 / & .4268 \\ 13.9 / & 14 / & .0474 \\ 34.8 / & 1 / & 8.77 \\ 1.5 / & 1 / & .39 \\ & 5.95 & \\ & 2.53 & \end{array}$

24.89

24.89
181.96

238.33

.3813

8.39

3.52

178.05

26.016

65.062

1636.97

3.052

.551

24.074

.606

$16.67 ; 14.11)$

$\mathrm{CH} 4 \quad \mathrm{G} / \mathrm{MI}$

NMFC G/MI

CARBONYL G/MI

ALCOHOL G/MI
RESEARCH

PROJECT NO. 08-6068-001 $\begin{array}{llll}\text { FUEL DENSITY } & 5.733 & \text { LB/GAL } \\ \text { H } .181 & \text { C } .819 & 0.000 & X .000\end{array}$

NOX HUMIDITY C.F. . .811

3 TRT TRANSIENT

( $0-505$ SEC.)

505.0

$.987 / .994$

$3.65(5.88)$

$571.9(16.20)$

$.27(.01)$

4815. ( 136.4$)$

$\begin{array}{rrr}18.9 / & 3 / & 188.58 \\ .8 / & 3 / & 7.98 \\ 79.4 / & 13 / & 191.31 \\ .5 / & 13 / & 1.09 \\ 74.7 / & 14 / & .5695 \\ 13.4 / & 14 / & .0454 \\ 31.0 / & 2 / & 31.01 \\ .2 / & 2 / & .20 \\ & 5.53 & \\ 2.54 & \end{array}$

19.26

19.26
181.01

186.43

.5265

30.82

3.12
177.55

25.244

29.598

1314.47

.518
.284

.284
13.962

.469

$20.26(11.61)$

3-BAG COMPOSITE RESULTS

$\begin{array}{llr}\text { THC } & \text { G/MI } & 5.495 \\ \text { CO } & \text { G/MI } & 11.980 \\ \text { NOX } & \text { G/MI } & 1.259\end{array}$

FUEL ECONOMY MPG (L/10OKM) 17.81 (13.21)

.107

5.063

.272 
SOUTHWEST RESEARCH INSTITUTE - DEPARTMENT OF EMISSIONS RESEARCH

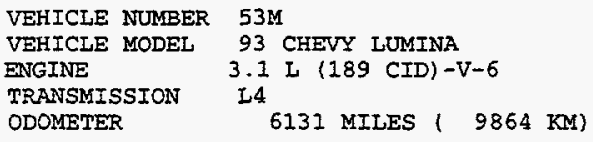

BAROMETER 29.62 IN HG $(752.3 \mathrm{MM}$ HG) RELATIVE HUMIDITY 21.6 PCT.

\section{BAG NUMBER}

BAG DESCRIPTION

RUN TIME SECONDS

DRY/WET CORRECTION FACTOR, SAMP/BACK

MEASURED DISTANCE MILES (KM)

BLOWER FLOW RATE SCFM (SCMM)

GAS METER FLOW RATE SCFM (SCMN)

TOTAL FLOW SCF (SCM)

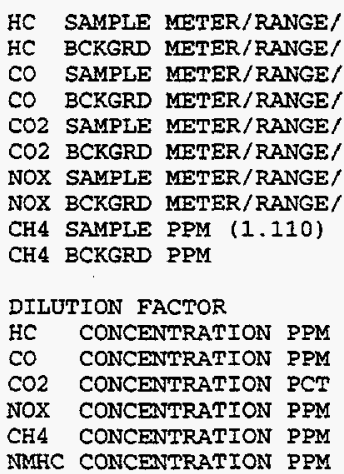

NMHC CONCENTRATION

$\begin{array}{ll}\text { THC } & \text { MASS GRAMS } \\ \text { CO } & \text { MASS GRAMS } \\ \text { CO2 } & \text { MASS GRAMS } \\ \text { NOX } & \text { MASS GRAMS } \\ \text { CH4 } & \text { MASS GRAMS } \\ \text { NMHC } & \text { MASS GRAMS (FID) } \\ \text { FUEI } & \text { MASS KG } \\ \text { FUEL ECONOMY MPG ( } / 1 / 100\end{array}$

3-BAG COMPOSITE RESULTS 3-BAG CARB FTP VEHICLE EMISSION RESULTS

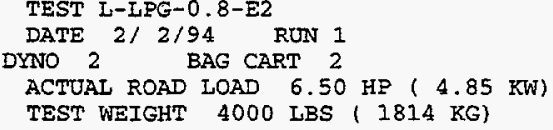

DRY BULB TEMPERATURE $72.0^{\circ} \mathrm{F}\left(22.2^{\circ} \mathrm{C}\right)$

$$
\begin{gathered}
1 \\
\text { COLD TRANSIENT } \\
(0-505 \text { SEC. }) \\
504.8 \\
.986 / .994 \\
3.66(5.88) \\
569.9(16.14) \\
.27(.01) \\
4797 .(135.9)
\end{gathered}
$$

$18.3 / 3 / 182.59$

$.8 / 3 / 7.98$

$47.0 / 13 / 107.96$

$.7 / 13 / 1.52$

79.21341 .5446

$14.8 / 14 / .0510$

$\begin{array}{rrr}14.8 / & 14 / & .0510 \\ 32.9 / & 2 / & 32.91\end{array}$

(BAG) (D)

.51

$2 / .50$

2.69

17.36

175.07

104.17

.5965

32.44

2.16

172.67

14.877

16.877

1483.71

6.836

.196

13.527

518

$18.35(12.82)$

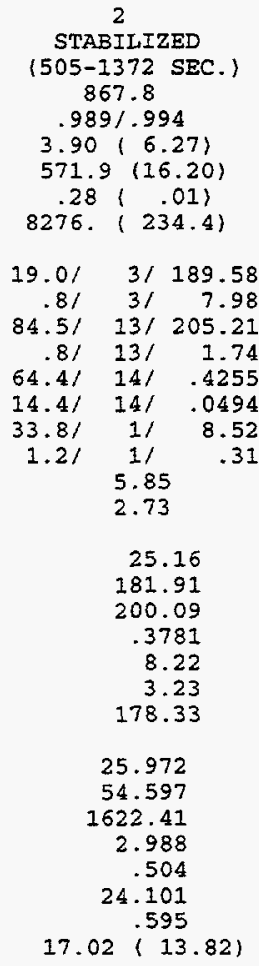

PROJECT NO. 08-6068-001
FUEL DENSITY 5.733 LB/GAL

$\mathrm{H} .181 \mathrm{C} .8190 .000 \times .000$

NOX HUMIDITY C.F. .811

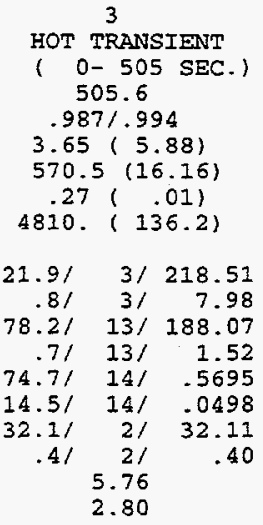

19.18

210.95

182.84

.5223

31.74

3.11

207.50

$$
17.539
$$

28.996

1302.55

6.705

282

15.299

.467

$20.36 ;(11.56)$

$\begin{array}{llr}\text { THC } & \text { G/MI } & 5.610 \\ \text { CO } & \text { G/MI } & 10.355 \\ \text { NOX } & \text { G/MI } & 1.291\end{array}$

FUEL ECONOMY MPG (L/100KM)

$\begin{array}{llr}\text { CH4 } & \text { G/MI } & .099 \\ \text { MMHC } & \text { G/MI } & 5.191 \\ \text { CARBONYL } & \text { G/MI } & .271 \\ \text { ALCOHOL } & \text { G/MI } & .048\end{array}$


VEFICLE NUMBER $53 \mathrm{M}$

VEHICLE MODET 93 CHEVY IUMINA

ENGINE

93 CHEVY IUMINA
3.1 L ( 189 CID $)-V-6$

$\mathrm{L} 4$

6083 MILES ( $9787 \mathrm{KM}$ )
3-BAG CARE FTP VEHICLE EMISSION RESULTS

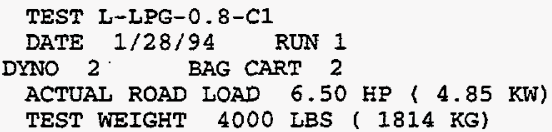

BAROMETER 29.40 IN HG $(746.8 \mathrm{MM}$ HG) RELATIVE HUMIDITY 19.8 PCT.

BAG NUMBER

BAG DESCRIPTION

RUN TIME SECONDS

DRY/WET CORRECTION FACTOR, SAMP/BACK

MEASURED DISTANCE MILES (KM)

BLOWER FLOW RATE SCFM (SCMM)

GAS METER FIOW RATE SCFM (SCMM)

TOTAL FLOW SCF (SCM)

HC SAMPLE METER/RANGE/PPM (BAG)

HC BCKGRD METER/RANGE/PPM

CO SAMPLE METER/RANGE/PPM

CO BCKGRD METER/RANGE/PPM

CO2 SAMPLE METER/RANGE/PCT

CO2 BCKGRD METER/RANGE/PCT

NOX BCKGRD METER/RANGE/PPM

CH4 SAMPLE PPM (1.110)

CH4 BCKGRD PPM

(BAG) (D) $\begin{array}{rrrr}13.3 / & 14 / & .0450 \\ & 39.2 / & 2 / & 39.22\end{array}$

DRY BULB TEMPERATURE $73.0^{\circ} \mathrm{F}\left(22.8^{\circ} \mathrm{C}\right)$

$$
\begin{gathered}
1 \\
\text { COLD TRANSIENT } \\
(0-505 \text { SEC. }) \\
505.2 \\
.986 / .994 \\
3.61(5.80) \\
568.2(16.09) \\
.14\left(\begin{array}{c}
.00) \\
4785 .(135.5)
\end{array}\right.
\end{gathered}
$$

$53.6 / 2 / \quad 53.57$

$5.1 / 2 / 5.10$

$15.6 / 12 / 15.01$

.91121 .85

.412

3.86

2.43

17.52

48.76

48.76
13.88

.6183

38.84

1.57

47.02

CHA CONCENTRATION PPM

NMHC CONCENTRATION PPM

$\begin{array}{lll}\text { THC } & \text { MASS GRAMS } \\ \text { CO } & \text { MASS GRAMS } \\ \text { CO2 } & \text { MASS GRAMS } \\ \text { NOX } & \text { MASS GRAMS } \\ \text { CH4 } & \text { MASS GRAMS } \\ \text { NMHC } & \text { MASS GRAMS (FID) } \\ \text { FUEL MASS KG } & \end{array}$

3.877

2.190

1533.99

8.126

.142

3.675

516

$18.16 ; 12.95\rangle$

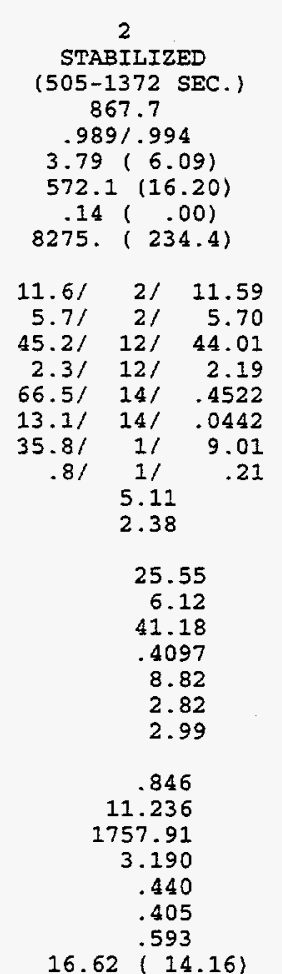

NOX HUMIDITY C.F. $\quad .807$

HOT TRANSIENT

( 0 - 505 SEC.

505.4

$.987 / .994$

$3.60(5.79)$

571.7 (16.19)

$.14(.00)$

4816. (136.4)

$\begin{array}{rrr}38.3 / & 2 / & 38.28 \\ 5.7 / & 2 / & 5.70 \\ 57.4 / & 12 / & 56.11 \\ .5 / & 12 / & .47 \\ 75.7 / & 14 / & .5855 \\ 12.9 / & 14 / & .0434 \\ 31.7 / & 2 / & 31.71 \\ .3 / & 2 / & .30 \\ & 4.68 & \\ 2.34 & \end{array}$

19.66

32.87

54.55

.5443

31.43

2.46

30.14

2.595

8.662

1359.16

6.619

.224

2.370

.460

$20.34\{11.56\}$

3-BAG COMPOSITE RESULTS

$\begin{array}{llr}\text { THC } & \text { G/MI } & .540 \\ \text { CO } & \text { G/MI } & 2.316 \\ \text { NOX } & \text { G/MI } & 1.415 \\ \text { FUET ECONOMY MPG } & \text { (I/100k }\end{array}$

$17.85(13.18)$

$\begin{array}{lll}\text { CH4 } & \text { G/MI } & .085 \\ \text { NMHC } & \text { G/MI } & .451 \\ \text { CARBONII } & \text { G/MI } & .004\end{array}$


SOUTHWEST RESEARCH INSTITUTE - DEPARTMENT OF EMISSIONS RESEARCH

3-BAG CARB FTP VEHICLE EMISSION RESULTS

PROJECT NO. 08-6068-001

$\begin{array}{lcl}\text { VEHICLE NUMBER } & 53 \mathrm{M} \\ \text { VEHICLE MODEL } & 93 \mathrm{CHEVY} \text { LUMINA } \\ \text { ENGINE } & 3.1 \mathrm{~L}(189 \mathrm{CID})-\mathrm{V}-6 \\ \text { TRANSMISSION } & \mathrm{L} 4 \\ \text { ODOMETER } & 6101 \text { MILES }(9816 \mathrm{KM})\end{array}$

BAROMETER 29.65 IN HG (753.1 MM HG) RELATIVE HUMIDITY 23.8 PCT.

BAG NUMBER

BAG DESCRIPTION

RUN TIME SECONDS

DRY/WET CORRECTION FACTOR, SAMP/BACK

MEASURED DISTANCE MILES (KM)

BLOWER FLOW RATE SCFM (SCMM)

GAS METER FLOW RATE SCFM (SCMM)

TOTAL FLOW SCF (SCM)

HC SAMPLE METER/RANGE/PPM (BAG)

HC BCKGRD METER/RANGE/PRM

CO SAMPLE METER/RANGE/PPM

CO BCKGRD METER/RANGE/PPM

CO2 SAMPLE METER/RANGE/PCT

CO2 BCKGRD METER/RANGE/PCT

NOX SAMPLE METER/RANGE/PPM (BAG) (D)

NOX BCKGRD METER/RANGE/PPM

CH4 SAMPLE PPM $(1.110)$

CH4 BCKGRD PPM

DILUTION FACTOR

HC CONCENTRATION PPM

CO CONCENTRATION PRM

$\mathrm{CO} 2$ CONCENTRATION PCT

NOX CONCENTRATION PPM

CH4 CONCENTRATION PRM

NMHC CONCENTRATION PPM

\section{THC MASS GRAMS \\ CO MASS GRAMS \\ $\mathrm{CO} 2$ MASS GRAMS \\ NOX MASS GRAMS \\ CH4 MASS GRAMS \\ NMAC MASS GRAMS (FID)}

FUEL MASS KG

FUEI ECONOMY MPG (I/100KM)

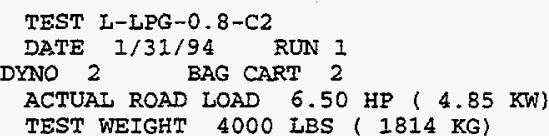

DRY BULB TEMPERATURE $71.0^{\circ} \mathrm{F}\left(21.7^{\circ} \mathrm{C}\right)$

COED TRANSIEANT

( $0-505$ SEC.)

504.7

$.985 / .994$

$3.65(5.88)$

$572.5(16.21)$

$.14(.00)$

4817 . (136.4)

$57.9 / 2 / \quad 57.87$

$5.6,21 \quad 5.60$

$13.7 / 12 / \quad 13.16$

$.7 / 12 / \quad .66$

$81.1 / 14 / .6790$

$13.7 / 14 / .0466$

$32.4 / 2 / 32.41$

$\begin{array}{lll}.41 & 21 & .40\end{array}$

3.49

2.32

17.04

52.60

12.23

.6352

32.04

1.30

51.15

4.206

1.943

1586.34

6.819

.119

4.023
.534

$17.79(13.22)$
STABILIZED

(505-1372 SEC.)

868.0

$.988 / .994$

$3.88(6.24)$

$574.4(15.27)$

$.14(.00)$

8312 . (235.4)

$\begin{array}{rrr}10.8 / & 2 / & 10.79 \\ 6.4 / & 2 / & 6.40 \\ 50.2 / & 12 / & 48.95 \\ .9 / & 12 / & .85 \\ 67.4 / & 14 / & .4640 \\ 13.7 / & 14 / & .0466 \\ 33.5 / & 1 / & 8.44 \\ 1.5 / & 1 / & .39 \\ & 4.55 & \\ & 2.35 & \end{array}$

24.89

4.65

47.24

.4192

8.07

2.29

2.11

.648

12.946

1806.92

2.964

.360

.286

.610

$16.54(14.22)$

CH4 G/MI

CARBONYL G/MI
IJPG

FUEL DENSITY 5.733 LB/GAE

H $.181 \quad \mathrm{C} .8190 .000 \quad \mathrm{X} .000$

NOX HUMIDITY C.F. .816

$$
3
$$

HOT TRANSIENT

( $0-505$ SEC.)

505.2

$.986 / .994$

$3.65(5.87)$

$574.0(16.26)$

$.14(.00)$

$4835 .(136.9)$

$38.8 / 2 / 38.78$

6.01216 .00

$59.4 / 12 / 58.12$

$.4 / 12 / \quad .38$

$\begin{array}{llll}76.4 / & 14 / & .5969\end{array}$

$13.4 / \quad 14 / \quad .0454$

$32.9 / 2 / 32.91$

$\begin{array}{lll}.31 & 21 & .30\end{array}$

4.40
2.33

19.28

33.09

56.51

.5538

32.63

2.19

30.66

2.622

9.007

1388.32

6.971

.200

2.420
.470

$20.17(11.66)$

3-BAG COMPOSITE RESULTS

$\begin{array}{lcr}\text { THC } & \text { G/MI } & .525 \\ \text { CO } & \text { G/MI } & 2.513 \\ \text { NOX } & \text { G/MI } & 1.311 \\ \text { FUEI ECONOMY } & \text { MPG } & (\mathrm{L} / 100 \mathrm{~K}\end{array}$

$17.69(13.30\}$ 
SOUTHWEST RESEARCH INSTITUTE - DEPARTMENT OF EMISSIONS RESEARCH

3-BAG CARB FTP VEHICLE EMISSION RESULTS

PROJECT NO. 08-6068-001

VEHICLE NUMBER 53M

VEFICLE MODEL 93 CHEVY LUMINA

ENGINE $\quad 3.1$ L (189 CID) $-V-6$

TRANSMISSION L4

ODOMETER 5945 MILES ( $9565 \mathrm{KM}$ )

BAROMETER 29.56 IN HG (750.8 MM HG)

RELATIVE HUMIDITY 18.3 PCT.

BAG NOMBER

BAG DESCRIPTION

RUN TIME SECONDS

DRY/WET CORRECTION FACTOR, SAMP/BACK

MEASURED DISTANCE MILES (KM)

BLOWER FLOW RATE SCFM (SCMM)

GAS METER FLOW RATE SCFM (SCMM)

TOTAL FLOW SCF (SCM)

HC SAMPLE METER/RANGE/PPM (BAG)

HC BCKGRD METER/RANGE/PPM

CO SAMPLE METER/RANGE/PPM

CO BCKGRD METER/RANGE/PPM

CO2 SAMPLE METER/RANGE/PCT

CO2 BCKGRD METER/RANGE/PCT

NOX SAMPLE METER/RANGE/PPM (BAG) (D)

NOX BCKGRD METER/RANGE/PPM

CH4 SAMPLE PPM $(1.110)$

CH4 BCKGRD PPM

\section{DILUTION FACTOR}

HC CONCENTRATION PPM

CO CONCENTRATION PPM

$\mathrm{CO} 2$ CONCENTRATION PCT

NOX CONCENTRATION PPM

CH4 CONCENTRATION PPM

NMHC CONCENTRATION PPM

$\begin{array}{ll}\text { THC } & \text { MASS GRAMS } \\ \mathrm{CO} & \text { MASS GRAMS } \\ \mathrm{CO} 2 & \text { MASS GRAMS } \\ \text { NOX } & \text { MASS GRAMS } \\ \text { CH4 } & \text { MASS GRAMS } \\ \text { NMMHC } & \\ \text { FUSS GRAMS } & \text { (FID) } \\ \text { FUEL ECONOMY MPG (L/10OKM }\end{array}$

FUEL ECONOMY MPG (L/100KM)

$$
\begin{aligned}
& \text { TEST L-IUG- } 1.0-E 1 \\
& \text { DATE } 1 / 18 / 94 \quad \text { RUN } 1 \\
& \text { DXNO } 200 \text { BAG CART } 2 \\
& \text { ACTUAL ROAD IOAD } 6.50 \mathrm{HP}(4.85 \mathrm{KW}) \\
& \text { TEST WEIGHT } 4000 \text { LBS }(1814 \mathrm{kG})
\end{aligned}
$$

DRY BULB TEMPERATURE $69.0^{\circ} \mathrm{F}\left(20.6^{\circ} \mathrm{C}\right)$

$$
\begin{gathered}
1 \\
\text { COLD TRANSIENT } \\
(0-505 \text { SEC. }) \\
506.2 \\
.987) .996 \\
3.64(5.85) \\
552.8(15.66) \\
.28(5.01) \\
4666 .(132.2)
\end{gathered}
$$$$
\begin{array}{rrr}
78.0 / & 2 / & 77.95 \\
5.5 / & 2 / & 5.50 \\
62.7 / & 1 / & 564.79 \\
.0 / & 1 / & .00 \\
78.1 / & 14 / & .6254 \\
13.5 / & 14 / & .0458 \\
46.1 / & 2 / & 46.12 \\
.3 / & 2 / & .30 \\
& 6.40 & \\
& 2.90
\end{array}
$$

16.98

16.98
72.78

72.78
553.27

.5823

45.84

68.71

5.940

85.121

1409.01

9.186

.323

5.236
520

$18.18(12.94)$

3-BAG COMPOSITE RESULTS

$\begin{array}{llr}\text { THC } & \text { G/MI } & 1.946 \\ \text { CO } & \text { G/MI } & 20.869 \\ \text { NOX } & \text { G/MI } & 2.185\end{array}$

FUEL ECONOMY MPG (I/100KM)

$18.19(12.93)$

STABIIIZED

(505-1372 SEC.)

868.2

$.990 / .996$

$3.86(6.22)$

$568.1(16.09)$

$28(.01)$

$8224 .(232.9)$

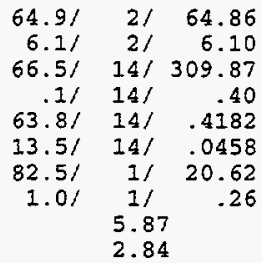

25.69
59.00

59.00
304.65

.3742

20.37

3.14

55.52

8.535

82.604

1595.47

7.195

.488

7.456
.584

$17.22(13.66)$

$\begin{array}{llr}\text { CH4 } & \text { G/MI } & .107 \\ \text { NMHC } & \text { G/MI } & 1.710 \\ \text { CARBONYI } & \text { G/MI } & .112 \\ \text { ALCOHOL } & \text { G/MI } & .017\end{array}$

NOX HUMIDITY C.F. .793

HOT TRANSIENT

( 0- 505 SEC.)

505.0

$988 / .996$

$3.63(5.84\rangle$

572.7 (16.22)

$28(.01)$

4823 . $(136.6)$

$\begin{array}{rrr}78.5 / & 2 / & 78.45 \\ 5.8 / & 2 / & 5.80 \\ 85.8 / & 14 / & 420.70 \\ .5 / & 14 / & 2.02 \\ 73.7 / & 14 / & -5539 \\ 13.7 / & 14 / & .0466 \\ 44.7 / & 2 / & 44.72 \\ .4 / & 2 / & .40 \\ & 6.04 & \\ 2.85 & \end{array}$

19.39

19.39
72.96

72.96
410.91

.5097

44.34

3.34

69.25

6.133

65.334

1274.61

9.183

.304
5.454

.354
.465

$20.29(11.60)$ 
SOUTHWEST RESEARCE INSTITUTE - DEPARTMENT OF EMISSIONS RESEARCH

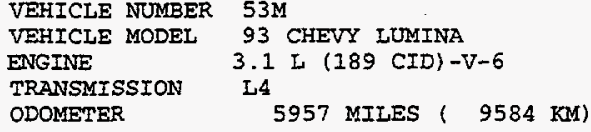

PROJECT NO. 08-6068-001

BAROMETER 29.59 IN HG $(751.6 \mathrm{MM} \mathrm{HG})$ RELATIVE HUMIDITY 31.2 PCT.

BAG NUMBER

BAG DESCRIPTION

RUN TIME SECONDS

DRY/WET CORRECTION FACTOR, SAMP/BACK

MEASURED DISTANCE MILES (KM)

BLOWER FLOW RATE SCFM (SCMM)

GAS METER FLOW RATE SCFM (SCMM)

TOTAL FLOW SCF (SCM)

HC SAMPLE METER/RANGE/PPM (BAG)
HC BCKGRD METER/RANGE/PPM
CO SAMPLE METER/RANGE/PPM
CO BCKGRD METER/RANGE/PPM
CO2 SAMPLE METER/RANGE/PCT
CO2 BCKGRD METER/RANGE/PCT .
NOX SAMPLE METER/RANGE/PPM (BAG) (D)
NOX BCKGRD METER/RANGE/PPM

CH4 SAMPLE PPM $(1.110)$

CH4 BCKGRD PPM

DILUIION FACTOR

$\begin{array}{ll}\text { HC CONCENTRATION PPM } \\ \text { CO } & \text { CONCENTRATION PPM } \\ \text { CO2 } & \text { CONCENTRATION PCT } \\ \text { NOX } & \text { CONCENTRATION PPM } \\ \text { CH4 } & \text { CONCENTRATION PPM } \\ \text { NMHC CONCENTRATION PPM }\end{array}$

\section{THC MASS GRAMS}

CO MASS GRAMS

$\mathrm{CO} 2$ MASS GRAMS

NOX MASS GRAMS

CH4 MASS GRAMS

NMHC MASS GRAMS (FID)

FUEI MASS KG

FUEI BCONOMY MPG (L/ $10 \mathrm{CKM}$ )

3-BAG COMPOSITE RESULTS

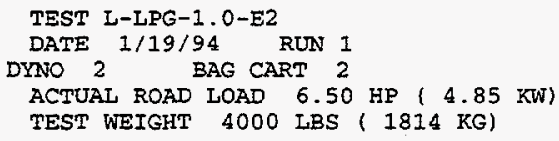

DRY BULB TEMPERATURE $68.0^{\circ} \mathrm{F}\left(20.0^{\circ} \mathrm{C}\right)$

$$
\begin{aligned}
& 1 \\
& \text { COID TRANSIENT } \\
& (0-505 \text { SEC. }) \\
& 505.0 \\
& .984 / .993 \\
& 3.64(5.86) \\
& 566.4(16.04) \\
& .28(1.01) \\
& 4769 .(135.1)
\end{aligned}
$$

$80.3 \%$

5.91

2/ 80.25

$66.3 /$

.11

215.90

$1 / 607.52$

$1 / \quad .69$

$78.8 / 14 / .6376$

$14.4 / 14 / .0494$

$\begin{array}{rrr}47.91 & 21 & 47.92 \\ .41 & 21 & .40\end{array}$

6.16
2.51

16.59

74.71

591.79

.5911

47.55

3.80

70.49

6.253

6.253
93.055

1461.80

10.217

.342

5.490

$17.47 ;(13.46)$

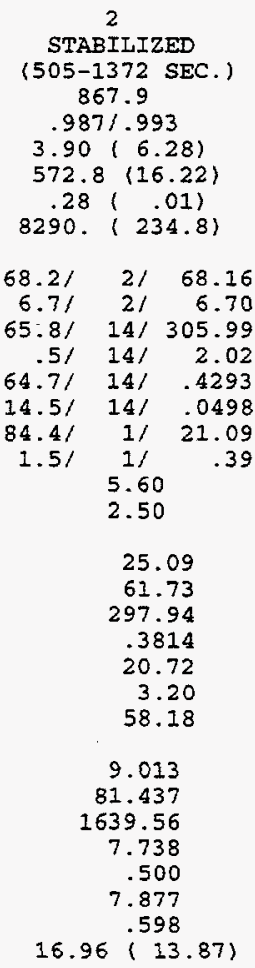

$14.9 / 14 / .0515$
IPG

FUEL DENSITY 5.733 LB/GAI

ت. .181 C .819 0.000 X .000

NOX HUMIDITY C.F. $\quad .832$

HOT TRANSIENT

( $0-505$ SEC.)

505.8

$.985 / .993$

$3.65(5.87)$

$568.1(16.09)$

$27(.01)$

4791. ( 135.7$)$

$80.4 / 2 / \quad 80.35$

$6.6121 \quad 6.60$

$87.1 / 14 / 428.32$

$.6 / 14 / 2.43$

$73.9 / 14 / 5570$

$\begin{array}{lll}45.8 / & 2 / & 45.82\end{array}$

.5121

5.73

2.50

19.27
74.10
416.20
.5082
45.35
3.36
70.37

6.340

65.748

1262.58

9.790

304

5.506

.462

$20.55 ;(11.44)$

$\begin{array}{llr}\text { THC } & \text { G/MI } & 2.029 \\ \text { CO } & \text { G/MI } & 21.057 \\ \text { NOX } & \text { G/MI } & 2.347\end{array}$

FUEL ECONOMY MPG (L/100KM)

$17.96(13.10)$

$\begin{array}{llr}\text { CH4 } & \text { G/MI } & .109 \\ \text { NMHC } & \text { G/MI } & 1.772 \\ \text { CARBONIL G/MI } & .121 \\ \text { ALCOHOL } & \text { G/MI } & .027\end{array}$


COMPUTER PROGRAM LDT $1.5-R$

VEHICLE NUMBER 53M

VEHICLE MODEL 93 CHEVY LUMINA

ENGINE

3.1 I (189 CID $)-V-6$

TRANSMISSION L4

ODOMETER

5902 MIIES ( $9496 \mathrm{KM}$ )

BAROMETER 29.31 IN HG (744.5 MM HG)

REIAATIVE HUMIDITY 60.7 PCT

BAG NUMBER

BAG DESCRIPTION

RUN TIME SECONDS

DRY/WET CORRECTION FACTOR, SAMP/BACK

MEASURED DISTANCE MILES (KM)

BLOWER FLOW RATE SCFM (SCMM)

GAS METER FLOW RATE SCFM (SCMM)

TOTAL FLOW SCF (SCM)

HC SAMPLE METER/RANGE/PPM (BAG)

HC BCKGRD NETER/RANGE/PPM

CO SAMPLE METER/RANGE/PPM

CO BCKGRD METER/RANGE/PPM

CO2 SAMPLE METER/RANGE/PCT

CO2 BCKGRD METER/RANGE/PCT

NOX SAMPLE METER/RANGE/PPM (BAG) (D)

NOX BCKGRD METER/RANGE/PPM

CH4 SAMPLE PPM $(1.110)$

CH4 BCKGRD PPM

\section{DILUTION FACTOR \\ HC CONCENTRATION PPM \\ CO CONCENTRATION PPM \\ $\mathrm{CO} 2$ CONCENTRATION PCT \\ NOX CONCENTRATION PPM \\ CH4 CONCENTRATTON PPM \\ NMHC CONCENTRATION PPM \\ THC MASS GRAMS \\ CO MASS GRAMS \\ $\mathrm{CO} 2$ MASS GRAMS \\ NOX MASS GRAMS \\ CH4 MASS GRAMS \\ NMHC MASS GRAMS (FID) \\ FUEI MASS KG}

FUEL ECONOMY MPG (I/100KM)
3-BAG CARB FTP VEHICLE EMISSION RESULTS
-BAT

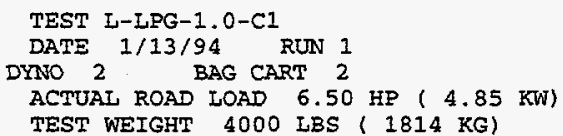

DRY BULB TEMPERATURE $54.0^{\circ} \mathrm{F}\left(17.8^{\circ} \mathrm{C}\right)$

$$
\begin{gathered}
1 \\
\text { COLD TRANSIENT } \\
(0-505 \text { SEC. } \\
505.3 \\
.979 / .987 \\
3.64(5.85) \\
566.7(16.05) \\
.28(1.01) \\
4775 .(135.2)
\end{gathered}
$$

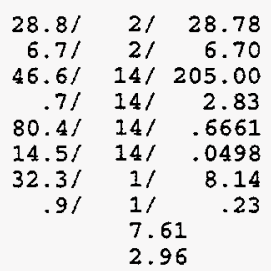

16.98

22.48

195.21

.6193

7.92

4.82

17.13

1.779

30.731

1533.13

1.874

.435

1.336

$17.89(13.15)$
STABILIZED

(505-1372 SEC.) 868.4

.9821 .987

$3.87(6.23)$

$567.1(16.06)$

$28(.01)$

$8212 .(232.6)$

\section{$14.0 / 2 / \quad 13.99$}

$8.9 / 21 \quad 8.89$

$43.0 / 12 / \quad 41.84$

$2.21 \quad 12 / 2.09$

$66.4 / \quad 14 / .4509$

$14.0 / \quad 14 / \quad .0478$

$17.6 / 1 / 4.47$

.5/ $1 / \quad .13$

2.82

\subsection{3}

5.44

38.61

.4049

4.35

. 5.02

$-.12$

.783
10.454

1724.15

1.768

.778

.000
.581

$17.34(13.57)$

RESEARCH

PROJECT NO. 08-6068-001

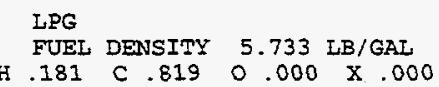

H $.181 \quad \mathrm{C} .8190 .000 \quad \mathrm{X} .000$

NOX HUMIDIMY C.E. . .914

HOT TRANSIENT

( 0- 505 SEC.

504.5

$.980 / .987$

$3.62(5.82)$

$565.9(16.03)$

$.28(.01)$

4760 . (134.8)

$18.9 / 2 / \quad 18.89$

$7.1 / 2 / 2.10$

$57.1 / 13 / 133.05$

$.9 / 13 / 1.96$

$75.7 / \quad 14 / \quad .5855$

$13.9 / 14 / \quad 0474$

$41.5 / 1 / 30.43$

$\begin{array}{lll}.71 & 11 & 10.43\end{array}$

6.08

2.78

19.48
12.16

126.81

.5405

10.26

3.44

8.34

.950

1334.05

2.419

.309
.648

.456

$20.62(11.41)$

3-BAG COMPOSITE RESULTS

$\begin{array}{llr}\text { THC } & \text { G/MI } & .279 \\ \text { CO } & \text { G/MI } & 4.668 \\ \text { NOX } & \text { G/MI } & .527\end{array}$

FUEL ECONOMY MPG
.527

$18.27(12.87)$

$\begin{array}{lll}\text { CH4 } & \text { G/MI } & .152 \\ \text { NNMC } & \text { G/MI } & .126 \\ \text { CARBONYL } & \text { G/MI } & .000 \\ \text { ALCOHOL } & \text { G/MI } & .001\end{array}$


COMPUTER PROGRAM LDT $1.5-\mathrm{R}$

EHICLE NUMBER $53 \mathrm{M}$

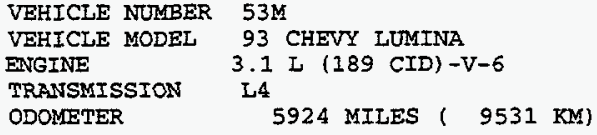

BAROMETER 29.38 IN HG (746.3 MM HG) RELATIVE HUMIDITY 41.5 PCT.

BAG NUMBER

BAG DESCRIPTION

RUN TIME SECONDS

DRY/WET CORRECTION FACTOR, SAMP/BACK

MEASURED DISTANCE MILES (KM)

BLOWER FLOW RATE SCFM (SCMM)

GAS METER FLOW RATE SCFM (SCMM)

TOTAL FLOW SCF (SCM)

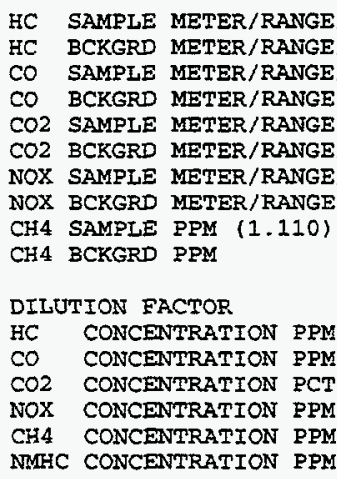

NMHC CONCENTRRATTON PPM

THC MASS GRAMS

CO MASS GRAMS

$\mathrm{CO} 2$ MASS GRAMS

NOX MASS GRAMS

CH4 MASS GRAMS

NMHC MASS GRAMS (FID)

FUEI MASS KG

FUEI ECONOMY MPG (I/100KM)

\section{(BAG)}

\section{M} (BAG) (D) (D) EST RESEARCH INSTITUTE - DEPARTMENT OF EMIS
3-BAG CARB FTP VEHICLE EMISSION RESULTS

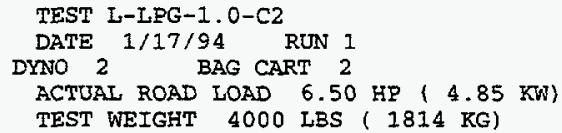

NOX HUMIDITY C.F. . .841

3 HOT TRANSIENNT

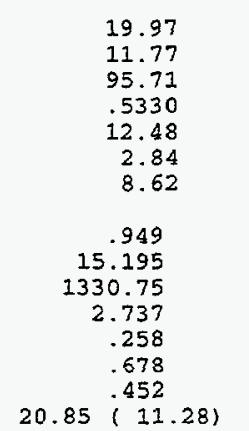

3-BAG COMPOSITE RESULTS

$\begin{array}{llr}\text { THC } & \text { G/MI } & .286 \\ \text { CO } & \text { G/MI } & 4.295 \\ \text { NOX } & \text { G/MI } & .670\end{array}$

$\begin{array}{lll}\text { CH4 } & \text { G/MI } & .123 \\ \text { NMHC } & \text { G/MI } & .158 \\ \text { CARBONIL G/MI } & .003 \\ \text { ALCOSOI } & \text { G/MI } & .001\end{array}$

CARBONIL G/MI

FUEL ECONOMY MPG (L/100KM) 18.24 (12.90) 
COMPUTER PROGRAM LDT $1.5-R$

VEHICLE NUMBER 53M

VEHICLE MODEL 93 CHEVY LUMINA

ENGINE

3.1 I $(189$ CID $)-V-6$

I.4

TRANSMISSION

ODOMETER $\quad 5997$ MILES ( $9649 \mathrm{KM}$ )

BAROMETER 29.66 IN HG (753.4 MM HG)

REIATIVE HUMIDTTY 48.9 PCT.

BAG NUMBER

BAG DESCRIPTION

RUN TIME SECONDS

DRY/WET CORRECTION FACTOR, SAMP/BACK

MEASURED DISTANCE MILES (KM)

BLOWER FLOW RATE SCFM (SCMM)

GAS METER FLOW RATE SCFM (SCMM)

TOTAL FLOW SCE (SCM)

HC SAMPLE METER/RANGE/PPM (BAG)

FC BCRGRD METER/RANGE/PPM

CO SAMPLE METER/RANGE/PPM

CO BCKGRD METER/RANGE/PPM

CO2 SAMPLE METER/RANGE/PCT

CO2 BCKGRD METER/RANGE/PCT

NOX SAMPLE METER/RANGE/PPM (BAG) (D)

NOX BCKGRD METER/RANGE/PPM

CH4 SAMPLE PPM $(1.110)$

CH4 BCKGRD PPM

\section{DILUTION FACTOR}

HC CONCENTRATION PPM

CO CONCENTRATION PPM

$\mathrm{CO} 2$ CONCENTRATION PCT

NOX CONCENTRATION PPM

CH4 CONCENTRATION PPM

NMHC CONCENTRATION PPM

THC MASS GRAMS

CO MASS GRAMS

$\mathrm{CO} 2$ MASS GRAMS

NOX MASS GRAMS

CH4 MASS GRAMS

NMHC MASS GRAMS (FID)

FUEI MASS KG

FUEL ECONOMY MPG (L/100KM)

(BAG)

3-BAG COMPOSITE RESULTS

$\begin{array}{llr}\text { THC } & \text { G/MI } & 2.805 \\ \text { CO } & \text { G/MI } & 93.746 \\ \text { NOX } & \text { G/MI } & .678\end{array}$

FUEL ECONOMY MPG (L/100KM)

$15.99(14.72)$

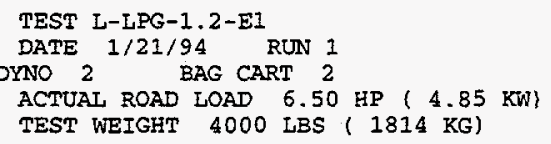

DRY BULB TEMPERATURE $66.0^{\circ} \mathrm{F}\left(18.9^{\circ} \mathrm{C}\right)$

$$
\begin{gathered}
1 \\
\text { COLD TRANSIENT } \\
(0-505 \text { SEC. } \\
505.2 \\
.983(.989 \\
3.60(5.79) \\
572.4(16.21) \\
.28(1.01) \\
4822 .(136.6)
\end{gathered}
$$

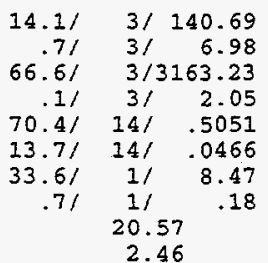

14.15

134.20

3074.42

.4618

8.30

18.28

113.91

10.940

488.774

1154.70

1.914

1. 664

8.969

652

$14.36(16.38)$

$$
\begin{gathered}
2 \\
\text { STABILIZED } \\
(505-1372 \text { SEC. }) \\
867.5 \\
.985 / .989 \\
3.82(6.15) \\
575.3(16.29) \\
.28(0.01) \\
8322 .(235.7)
\end{gathered}
$$

$88.8 / 2 / \quad 88.75$

$7.2 / 2 / 7.20$

$\begin{aligned} 7.2 / & 2 / \\ 64.8 / & 2 / 1471.87\end{aligned}$

$.272 / 2.68$

$58.9 / 14 / \quad 3615$

$13.4 / 14 / .0454$

$25.3 / \quad 1 / \quad 6.40$

$\begin{array}{lll}.6 / 11 & 11 & .15\end{array}$

11.92
2.45

22.76

81.87

1433.79

.3181

6.25

9.58

71.23

11.507

393.390

1372.47

2.488

1.506

9.680

.675

$14.73(15.97)$

CH4 G/MT

NMHC $\quad G / M I$

CARBONYL G/MI

ALCOHOL G/MI
RESEARC

PROJECT NO. 08-6068-001

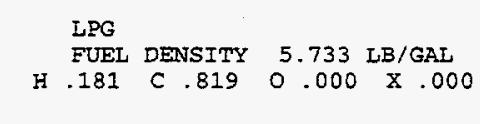

NOX HUMIDITY C.F. $\quad .883$

$$
\begin{gathered}
3 \\
\text { HOT TRANSIENT } \\
(0-505 \text { SEC. } \\
504.4 \\
.983(.989 \\
3.60(5.80) \\
578.6(16.39) \\
.28(1.01) \\
4867 .(137.8)
\end{gathered}
$$

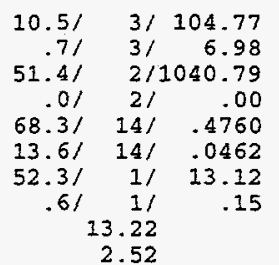

$$
\begin{array}{r}
19.90 \\
98.13 \\
1012.89 \\
.4322 \\
12.98 \\
10.83 \\
86.12
\end{array}
$$

8.111

162.525

1090.50

3.021

.995

6.844

.457

$20.52(11.46)$ 
SOUTHWEST RESEARCH INSTITUTE - DEPARTMENT OF EMISSIONS RESEARCH

VEHICLE NUMBER 53M

VEHICLE MODEL 93 CHEVY LUMINA

ENGINE

3.1 I (189 CID) $-\mathrm{V}-6$

TRANSMISSION IS

ODOMETER

6016 MILES ( $9679 \mathrm{KM}$ )

BAROMETER 29.33 IN HG (745.0 MM HG) RELATIVE HUMIDITY 60.1 PCT.

BAG NUMBER

BAG DESCRIPTION

RUN TIME SECONDS

DRY/WET CORRECTION FACTOR, SAMP/BACK

MEASURED DISTANCE MILES (KM)

BLOWER FLOW RATE SCFM (SCMM)

GAS METER FLOW RATE SCFM (SCMM)

TOTAL FLOW SCF (SCM)

HC SAMPLE METER/RANGE/PPM (BAG)

HC BCKGRD METER/RANGE/PPM

CO SAMPLE METER/RANGE/PPM

CO2 SAMPLE METER/RANGE/PCT

CO2 BCKGRD METER/RANGE/PCT

NOX SAMPLE METER/RANGE/PPM

NOX BCKGRD METER/RANGE/PPM

CH4 SAMPLE PPM (1.110)

CH4 BCKGRD PPM

\section{DILUTION FACTOR}

HC CONCENTRATION PPM

CO CONCENTRATION PPM

$\mathrm{CO} 2$ CONCENTRATION PCT

NOX CONCENTRATION PPM

CH4 CONCENTRATION PPM

NMHC CONCENTRATION PPM

$\begin{array}{lll}\text { THC } & \text { MASS GRAMS } \\ \text { CO } & \text { MASS GRAMS } \\ \text { CO2 } & \text { MASS GRAMS } \\ \text { NOX } & \text { MASS GRAMS } \\ \text { CH4 } & \text { MASS GRAMS } \\ \text { NMHC } & \text { MASS GRAMS } & \text { (FID) } \\ \text { FUEL } & \text { MASS KG } & \end{array}$

FUEL ECONOMY MPG (L/100KM)
3-BAG CARB FTP VEHICLE EMISSION RESULTS

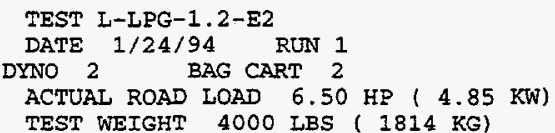

DRY BULB TEMPERATURE $78.0^{\circ} \mathrm{F}\left(25.6^{\circ} \mathrm{C}\right)$

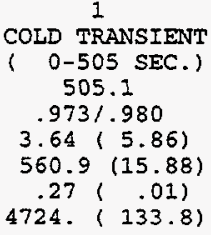

$15.4 / 3 / 153.66$

$\begin{array}{rrr}9 / 4 / 3 & 3 / 26 \\ 6 / 3 & 3.98\end{array}$

$65.6 / 3 / 3164.95$

$.1 / 3 / 2.33$

$70.9 / 14 / \quad 5123$

$13.5 / 14 / .0458$

(BAG) (D)

$28.1 / 1 / 7.10$
$.21 \quad 1 / \quad .05$

22.24

14.02

14.02
145.32

145.32
3063.86

.4698

7.05

20.04

123.08

11.645

477.198

1150.63

1.924

1.787

9.494

645

$14.68(16.03)$

STABILIZED

(505-1372 SEC.) 867.7

$.975 / .980$

$3.88(6.24)$

$563.7(15.96)$

$.27(.01)$

8156 . (231.0)

$96.2 / 2 / 96.14$

$9.6 / 2 / 29.59$

$66.4 / 2 / 1570.89$

$.4 / 2 / \quad 5.26$

$85.2 / 13 / \quad .3577$

$18.7 / 13 / .0436$

$19.6 / \quad 1 / 4.97$

$.3 / 1 / 12.08$

2.37

22.49

86.98

1521.52

.3161

4.90

10.85

74.93

12.063

409.121

1336.62

2.308

1. 671

9.979

$15.02(15.66)$

PROJECT NO. 08-6068-001

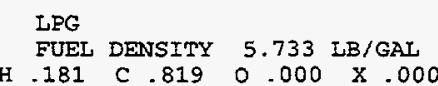

NOX HUMIDITY C.F. 1.067

$$
\begin{aligned}
& \text { HOT TRANSIENT } \\
& (0-505 \text { SEC. }) \\
& 505.3 \\
& .974(.980 \\
& 3.62(5.82) \\
& 560.0(15.86) \\
& .28(1.01) \\
& 4718 .(133.6)
\end{aligned}
$$

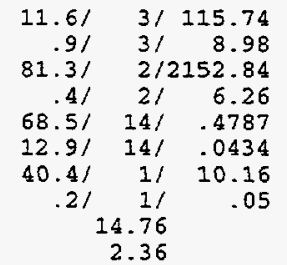

16.73

107.30

2081.43

.4379

10.11

12.54

93.38

8.571

323.776

1071.27

2.756

1.117
7.194

.535

$17.58(13.38)$

3-BAG COMPOSITE RESULTS

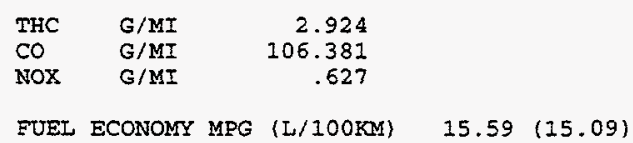

FUEL ECONOMY MPG $\{\mathrm{L} / 100 \mathrm{KM}\}$ 
COMPUTER PROGRAM INDT $1.5-\mathrm{R}$

VEHICLE NUMBER $53 \mathrm{M}$

VEHICLE MODEL 93 CHEVY LUMINA

ENGINE

93 CHEVY LUMINA
3.1 I $(189$ CID $)-V-6$

L4

ODOMETER $\quad 6034$ MILES ( $9708 \mathrm{KM}$ )

BAROMETER 29.09 IN HG $(738.9 \mathrm{MM} \mathrm{HG)}$

RELATIVE HUMIDITY 59.7 PCT.

BAG NUMBER

BAG DESCRIPTION

RUN TIME SECONDS

DRY/WET CORRECTION FACTOR, SAMP/BACK

MEASURED DISTANCE MILES (KM)

BLOWER FLOW RATE SCFM (SCMM)

GAS METER FLOW RATE SCFM (SCMM)

TOTAL FLOW SCF (SCM)

HC SAMPLE METER/RANGE/PPM (BAG)

HC BCKGRD METER/RANGE/PPM

CO SAMPLE METER/RANGE/PPM

CO BCKGRD METER/RANGE/PPM

CO2 SAMPLE METER/RANGE/PCT

CO2 BCKGRD METER/RANGE/PCT

NOX SAMPLE METER/RANGE/PPM (BAG) (D)

NOX BCKGRD METER/RANGE/PPM

CH4 SAMPLE PPM $(1.110)$

CH4 BCKGRD PPM

\section{DILUTION FACTOR}

HC CONCENTRATION PPM

CO CONCENTRATION PPM

$\mathrm{CO} 2$ CONCENTRATION PCT

NOX CONCENTRATION PPM

CH4 CONCENTRATION PPM

NMHC CONCENTRATION PPM

$\begin{array}{ll}\text { THC } & \text { MASS GRAMS } \\ \text { CO } & \text { MASS GRAMS } \\ \text { CO2 } & \text { MASS GRAMS } \\ \text { NOX } & \text { MASS GRAMS } \\ \text { CH4 } & \text { MASS GRAMS } \\ \text { NMHC } & \text { MASS GRAMS (FID) } \\ \text { FUEL MASS KG } & \end{array}$

FUEL MASS KG

FUEL ECONOMY MPG (L/100K

$\begin{array}{llr}\text { THC } & \text { G/MI } & 2.145 \\ \text { CO } & \text { G/MI } & 92.709 \\ \text { NOX } & \text { G/MI } & .025\end{array}$

3-BAG COMPOSITE RESULTS

FUEL ECONOMY MPG (L/10OKM) 15.48 (15.19)

3-BAG CARB FTP VEHICLE EMISSION RESULTS

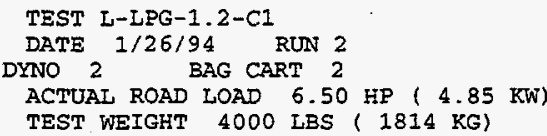

DRY BULB TEMPERATURE

$77.0^{\circ} \mathrm{F}\left(25.0^{\circ} \mathrm{C}\right)$

$$
\begin{gathered}
\text { I } \\
\text { COID TRANSIENT } \\
(0-505 \text { SEC. } \\
505.0 \\
.974 / .981 \\
3.63(5.84) \\
559.8(15.85) \\
.14(1.00) \\
4713 .(133.5)
\end{gathered}
$$

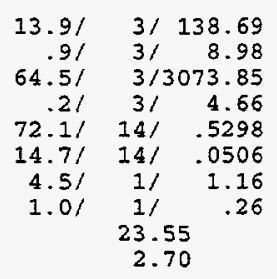

13.90

130.36

2972.63

.4828

.92
21.04

107.00

10.156

461.894

1179.74

.246

1.872

8.235

.645

$14.63(16.08)$

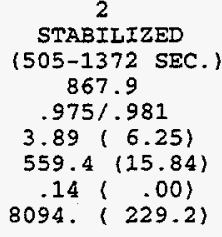

71.91

8.91
58.71

$2 / 1310.51$

$.4 / 2 / \quad 6.26$

$62.5 / 14 / .4025$

$14.5 / 14 / .0498$

$1.3 / \quad 1 / \quad .34$

$.8 / 1 / \quad .21$

15.75

21.78

63.37

1263.17

.3550

.14

14.11

47.71

8. 468

338.159

1489.78

.064

2.156

6.306

.682

$14.82(15.88)$
RESEARCH

PROJECT NO. $408-6068-00$

LPG

FUEL DENSITY $5.733 \mathrm{LB} / \mathrm{GAI}$

H .181 C $.819 \quad 0.000 \quad$ X .000

NOX HUMIDITY C.F. 1.052

3

HOT TRANSIENT

( $0-505$ SEC.)

505.6

$974 / .981$

$3.63(5.83)$

$559.8(15.85)$

$14(.00)$

4718 . ( 133.6$)$

$\begin{array}{lll}82.1 / 2 / 28.05 & 2 / 2\end{array}$

$74.412 / 1870.45$

$\begin{array}{rrr}4.47 & 2 / 1870.45 \\ .61 & 21 & 9.40\end{array}$

$71.0 / 14 / .5137$

$14.2 / 14 / .0486$

$1.0 / 1 / \quad .26$

$.6 / 1 / \quad .15$

18.04

2.87

16.63

73.97

1803.46

.4680

.11
15.35

56.94

5.761

280.547

1145.02

.030

1.367

4.387

.534

$17.64(13.33)$

$\begin{array}{llr}\text { CH4 } & \text { G/MI } & .498 \\ \text { NMHC } & \text { G/MI } & 1.643 \\ \text { CARBONYL } & \text { G/MI } & .004\end{array}$ 
SOUTHWEST RESEARCH INSTITUTE - DEPARTMENT OF EMISSIONS RESEARCH

3-BAG CARB FTP VEHICLE EMISSION RESULTS

PROJECT NO. 08-6068-001

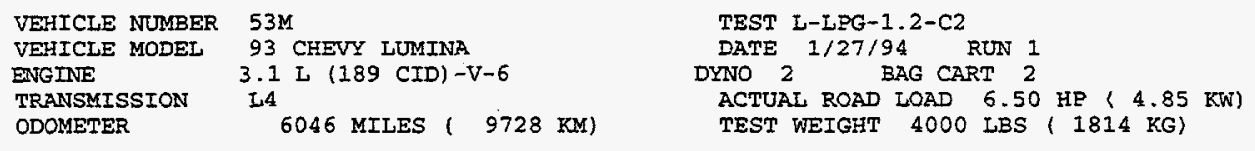

BAROMETER 28.95 IN HG $(735.3 \mathrm{MM} \mathrm{HG)}$ REIATIVE HUMIDITY 66.3 PCT.

DRY BULB TEMPERATURE $75.0^{\circ} \mathrm{F}\left(23.9^{\circ} \mathrm{C}\right)$

LPG

FUEL DENSITY 5.733 LB/GAI

$\begin{array}{lllllll}\mathrm{H} & .181 & \mathrm{C} & .819 & 0 & .000 & \mathrm{X} .000\end{array}$

BAG NUMBER

BAG DESCRIPTION

RUN TIME SECONDS

DRY/WET CORRECTION FACTOR, SAMP/BACK

MEASURED DISTANCE MILES (KM)

BLOWER FLOW RATE SCFM (SCMM)

GAS METER FLOW RATE SCFM (SCMM)

TOTAL FLOW SCF (SCM)

HC SAMPLE METER/RANGE/PPM (BAG)

HC BCKGRD METER/RANGE/PPM

CO SAMPLE METER/RANGE/PPM

CO BCKGRD METER/RANGE/PPM

CO2 SAMPLE METER/RANGE/PCT

$\mathrm{CO} 2$ BCKGRD METER/RANGE/PCT

NOX SAMPLE METER/RANGE/PPM (BAG) (D)

NOX BCKGRD METER/RANGE/PPM

CH4 SAMPLE PPM $(1.110)$

CH4 BCKGRD PPM

$$
\begin{gathered}
1 \\
\text { COLD TRANSIENT } \\
(0-505 \text { SEC. }) \\
505.0 \\
.973 / .980 \\
3.64(5.86) \\
555.8(15.74) \\
.14(1.00) \\
4679 .(132.5)
\end{gathered}
$$

$14.5 / 3 / 144.68$

$\begin{array}{rrr}14.5 / & 3 / & 144.68 \\ .9 / & 3 / & 8.98\end{array}$

$65.4 / 3 / 3148.26$

$.3 / 3 / 7.00$

$71.4 / \quad 14 / \quad .5195$

$13.5 / 14 / \quad .0458$

$4.0 / 1 / 1.03$

$.21 \quad 1 / \quad .05$

23.84

3.06

13.95

136.34

3036.58

.4770

.98

21.01

113.02

10.550

468.431

1157.23

.266

1.856
8.636

.642

$14.75(15.94)$

FUEL ECONOMY MPG (L/100KM)
MASS GRAMS

MASS GRAMS

MASS GRAMS

MASS KG
STABILIZED

(505-1372 SEC.) 868.1

$.975 / .980$

$3.89(6.26)$

$557.1(15.78)$

$14(.00)$

$8063 .(228.3)$

$\begin{array}{rcr}77.7 / & 2 / & 77.65 \\ 8.7 / & 2 / & 8.69 \\ 61.7 / & 2 / 1408.77 \\ .5 / & 2 / & 7.83 \\ 61.1 / & 14 / & .3862 \\ 13.3 / & 14 / & .0450 \\ .8 / & 1 / & .21 \\ .0 / & 1 / & .00 \\ & 15.97 & \\ & 2.87 & \end{array}$

22.05

69.35

1358.68

.3432

13.21

13.22
54.68

9.217

361.179

1434.84

.097

2. 013

7.199

.677

$14.94(15.74)$

$\mathrm{CH} 4$

G/MI

$G / M I$

CARBONYL G/MI
NOX HUMIDITY C.F. 1.073

HOT TRANSIENT

( $0-505$ SEC.

$$
505.2
$$

$.973 / .980$

$3.64(5.85)$

555.0 (15.72)

$.14(.00)$

$4674 .(132.4)$

$89.4 / 2 / \quad 89.35$

$8.4 / 21 \quad 8.40$

$76.7 / 2 / 1962.13$

$\begin{array}{ll}.6 / 2 / 2 & 2 / 1960\end{array}$

$\begin{array}{llll}70.4 / & 14 / & .5051\end{array}$

$13.8 / 14 / \quad .0470$

$.9 / \quad 1 / \quad .23$

$.3 / \quad 1 / \quad .08$

2.72

16.62

81.46

1888.49

.4610

.16

15.20
64.59

6.275

291.042

1117.24

.043

1.341

4. 930

$17.81(13.21)$

3-BAG COMPOSITE RESULTS

$\begin{array}{llclll}\text { THC } & \text { G/MI } & 2.303 & & \\ \text { CO } & \text { G/MI } & 96.791 & & \\ \text { NOX } & \text { G/MI } & .031 & & \\ \text { FUEL } & \text { ECONOMY MPG } & (\mathrm{L} / 100 \mathrm{KM}) & 15.62 & (15.06)\end{array}$

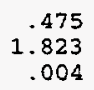




\section{APPENDIX D}

\section{COMPUTER PRINTOUTS OF EMISSIONS DATA FROM FTPs WITH CNG}

\begin{tabular}{|c|l|l|l||}
\hline Page D- & \multicolumn{1}{|c|}{ Test Number } & $\begin{array}{l}\text { Operating } \\
\text { Condition }\end{array}$ & \multicolumn{1}{|c|}{$\begin{array}{c}\text { Catalyst } \\
\text { Installation }\end{array}$} \\
\hline \hline 1 & L-CNG-0.8-E1 & Lean & Without Catalyst \\
\hline 2 & L-CNG-0.8-E2 & Lean & Without Catalyst \\
\hline 3 & L-CNG-0.8-C1 & Lean & With Catalyst \\
\hline 4 & L-CNG-0.8-C2 & Lean & With Catalyst \\
\hline \hline 5 & L-CNG-1.0-E2 & Stoich & Without Catalyst \\
\hline 6 & L-CNG-1.0-E3 & Stoich & Without Catalyst \\
\hline 7 & L-CNG-1.0-C1 & Stoich & With Catalyst \\
\hline 8 & L-CNG-1.0-C2 & Stoich & With Catalyst \\
\hline \hline 9 & L-CNG-1.2-E1 & Rich & Without Catalyst \\
\hline 10 & L-CNG-1.2-E2 & Rich & Without Catalyst \\
\hline 11 & L-CNG-1.2-C1 & Rich & With Catalyst \\
\hline 12 & L-CNG-1.2-C2 & Rich & With Catalyst \\
\hline
\end{tabular}


COMPUTER PROGRAM LDT $1.5-\mathrm{R}$

VEHICLE NUMBER $53 \mathrm{M}$

VEHICLE MODEI 93 CHEVY LUMINA

ENGINE

3.1 I (189 CID) $-\mathrm{V}-6$

BAROMETER 28.98 IN HG (736.1 MM HG)

REIATIVE HUMIDITY 78.0 PCT.

BAG NUMBER

BAG DESCRIPTION

RUN TIME SECONDS

DRY/WET CORRECTION FACTOR, SAMP/BACK

MEASURED DISTANCE MILES (KM)

BLOWER FLOW RATE SCFM (SCMM)

TOTAL FLOW SCF (SCM)

HC SAMPLE METER/RANGE/PPM (BAG)

HC BCKGRD METER/RANGE/PRM

CO SAMPLE METER/RANGE/PPM

CO BCKGRD METER/RANGE/PPM

CO2 SAMPLE METER/RANGE/PCT

CO2 BCKGRD METER/RANGE/PCT

NOX SAMPLE METER/RANGE/PPM (BAG) (D)

NOX BCKGRD METER/RANGE/PPM

CH4 SAMPLE PPM $(1.100)$

CH4 BCKGRD PPM

DILUTION FACTOR.

HC CONCENTRATION PPM

CO CONCENTRATION PPM

$\mathrm{CO} 2$ CONCENTRATION PCT

NOX CONCENTRATION PPM

CH4 CONCENTRATION PPM

NMHC CONCENTRATION PPM

$\begin{array}{lll}\text { THC } & \text { MASS GRAMS } \\ \text { CO } & \text { MASS GRAMS } \\ \text { CO2 } & \text { MASS GRAMS } & \\ \text { NOX } & \text { MASS GRAMS } & \\ \text { CH4 } & \text { MASS GRAMS } & \\ \text { NMHC } & \text { MASS GRAMS } & \text { (FID) } \\ \text { FUEI } & \text { MASS KG } & \end{array}$

FUEL ECONOMY MPG (L/100KM)

(BAG) (DAG)

TRANSMISSION I4

ODOMETER 6530 MILES ( $10506 \mathrm{KM}$ )

GAS METER FLOW RATE SCFM (SCMM)

\begin{abstract}
3-BAG CARB FTP VEHICLE EMISSION RESULTS
3-BATE INSTITUE - DERARTMENT OF EM
\end{abstract}

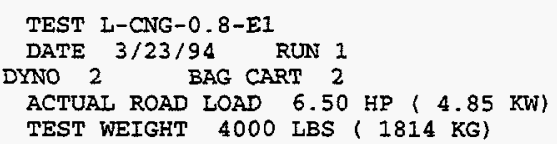

DRY BULB TEMPERATURE $74.0^{\circ} \mathrm{F}\left(23.3^{\circ} \mathrm{C}\right)$

$$
\begin{gathered}
1 \\
\text { COLD TRANSIENT } \\
(0-505 \text { SEC. }) \\
505.1 \\
.965 / .977 \\
3.62(5.83) \\
558.7(15.82) \\
.27(1.01) \\
4705 .(133.3)
\end{gathered}
$$

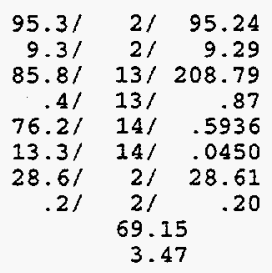

15.51

15.51
86.55

86.55
199.08

.5515

28.43

65.90

14.06

7.216
30.888

30.888

1345.63

8.294

5.855

1. 080

$17.98 ; 13.09)$

3-BAG COMPOSITE RESULTS

$\begin{array}{lll}\text { THC } & \text { G/MI } & 2.600 \\ \text { CO } & \text { G/MI } & 9.697 \\ \text { NOX } & \text { G/MI } & 1.469\end{array}$

FUEL ECONOMY MPG (L/100kM)

$18.05(13.03)$

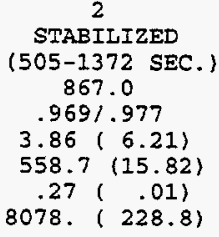

2

STABILIZED

(505-1372 SEC.) 867.0

.9691 .977

$3.86(6.21)$

$558.7(15.82)$

$27(.01)$

8078 . ( 228.8$)$

$\begin{array}{rrr}10.0 / & 3 / & 99.78 \\ 1.0 / & 3 / & 9.98 \\ 81.8 / & 13 / & 197.82 \\ .4 / & 13 / & .87 \\ 62.1 / & 14 / & .3978 \\ 13.2 / & 14 / & .0446 \\ 28.1 / & 1 / & 7.10 \\ .8 / & 1 / & .21 \\ & 73.18 & \\ & 3.40 & \end{array}$

22.65

22.65
90.24

189.71

.3552

6.90

69.93

13.32

12.870

50.524

1487.53

3.455

10.665

1. 756

$16.91(13.91)$

$\begin{array}{ll}\text { CH4 } & G / M I \\ \text { NMHC } & G / M I \\ \text { CARBONYL } & \text { G/MI }\end{array}$

ALCOHOL G/MI
PROJECT NO. 08-6068-001

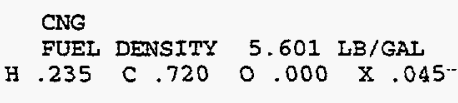

NOX HUMIDITY C.F. 1.145

$$
\begin{gathered}
3 \\
\text { HOT TRANSIENT } \\
(0-505 \text { SEC. }) \\
505.2 \\
.967) .977 \\
3.64(5.86) \\
558.3(15.81) \\
.27(1.01) \\
4703 .(133.2)
\end{gathered}
$$

$$
\begin{array}{rrr}
81.3 / & 2 / & 81.25 \\
9.3 / & 2 / & 9.29 \\
45.7 / & 13 / & 104.79 \\
.4 / & 13 / & .87 \\
72.3 / & 14 / & .5328 \\
13.1 / & 14 / & .0442 \\
24.2 / & 2 / & 24.21 \\
.3 / & 2 / & .30 \\
57.66 & \\
3.05 &
\end{array}
$$

17.54

72.49

99.71

.4911

23.93

54.78

54.78
12.23

6.140

15.461

1197.58

6.978

4.865

.939

$20.63 i^{448}(11.40)$ 
SOUTHWEST RESEARCH INSTITUTE - DEPARTMENT OF EMISSIONS RESEARCH

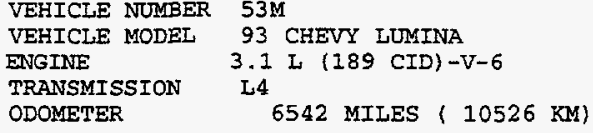

3-BAG COMPOSITE RESULTS

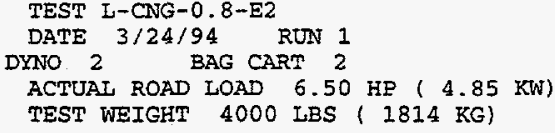

DRY BULB TEMPERATURE $78.0^{\circ} \mathrm{F}\left(25.6^{\circ} \mathrm{C}\right)$

$$
\begin{aligned}
& \text { COLD TRANSIENT } \\
& \text { (0-505 SEC.) } \\
& 505.0 \\
& .963 / .975 \\
& 3.61(5.81) \\
& 560.7 \text { (15.88) } \\
& .27\{.01\} \\
& \text { 4722. (133.7) } \\
& 10.2 / 3 / 101.77 \\
& 1.2 / 3 / 11.97 \\
& 87.4 / 13 / 213.21 \\
& .21 \quad 13 / \\
& 75.8 / 14 / .5873 \\
& 13.0 / 14 / \quad .0438 \\
& 26.6 / 2 / 26.61 \\
& \text {.3/ } 2 / \\
& 73.87 \\
& 3.69 \\
& 15.65 \\
& 90.57 \\
& 203.96 \\
& .5461 \\
& 26.33 \\
& 70.41 \\
& 13.11 \\
& 7.573 \\
& \begin{array}{r}
7.573 \\
31.752
\end{array} \\
& 1336.94 \\
& 8.150 \\
& 6.277 \\
& \text { 1. } 011 \\
& .509 \\
& 18.01 ;(13.06)
\end{aligned}
$$

$$
\begin{aligned}
& 2 \\
& \text { STABILIZED }
\end{aligned}
$$

PROJECT NO. 08-6068-001

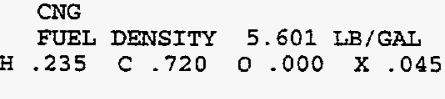

H.235 C .720 $0.000 \times .045$

NOX HUMIDITX C.F. 1.210

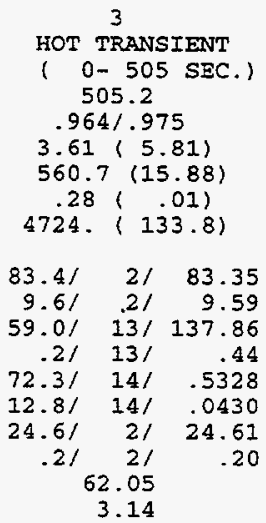

$\begin{array}{lllllr}\text { THC } & \text { G/MI } & 2.543 & \text { CH4 } & \text { G/MI } & 2.126 \\ \text { CO } & \text { G/MI } & 9.560 & \text { NNMC } & \text { G/MI } & .317 \\ \text { NOX } & \text { G/MI } & 1.527 & \text { CARBONYI G/MI } & .100 \\ & & & \text { ALCOHOI G/MI } & .000\end{array}$


COMPUTER PROGRAM LDT $1.5-\mathrm{R}$

SOUTHWEST RESEARCH INSTITUTE - DEPARTMENT OF EMTSSIONS RESEARCH

3-BAG CARB FTP VEHICLE EMISSION RESULTS

VEHICLE NUMBER 53M

VEHICLE MODEL 93 CHEVY LUMINA

ENGINE

3.1 I (189 CID) $-\mathrm{V}-6$

L4

ODOMETER $\quad 6498$ MILES ( $10455 \mathrm{KM})$

BAROMETER 29.18 IN HG (741.2 MM HG)

RELATIVE HUMIDITY 49.7 PCT

BAG NUMBER

BAG DESCRIPTION

RUN TIME SECONDS

DRY/WET CORRECTION FACTOR, SAMP/BACK

MEASURED DISTANCE MILES (KM)

BLOWER FLOW RATE SCFM (SCMM)

GAS METER FLOW RATE SCFM (SCMM)

TOTAL FIOW SCF (SCM)

\begin{tabular}{|c|c|c|}
\hline $\mathrm{HC}$ & SAMPLE METER/RANGE/PPM & (BAG) \\
\hline $\mathrm{HC}$ & BCKGRD METER/RANGE/PPM & \\
\hline Co & SAMPLE METER/RANGE/PPM & \\
\hline$c 0$ & BCKGRD METER/RANGE/PPM & \\
\hline $\mathrm{CO} 2$ & SAMPLE METER/RANGE/PCT & \\
\hline $\mathrm{CO} 2$ & BCKGRD METER/RANGE/PCT & \\
\hline$O x$ & SAMPLE METER/RANGE/PPM & $\langle B A G\rangle\langle D\rangle$ \\
\hline NOX & BCKGRD METER/RANGE/ PPM & \\
\hline $\mathrm{CH} 4$ & SAMPLE PPM $(1.100)$ & \\
\hline $\mathrm{CH} 4$ & BCKGRD PPM & \\
\hline \multicolumn{3}{|c|}{ DILUTION FACTOR } \\
\hline HC & CONCENTRATION PPM & \\
\hline$\infty$ & CONCENTRATION PPM & \\
\hline $\mathrm{CO} 2$ & CONCENTRATION PCT & \\
\hline NOX & CONCENTRATION PPM & \\
\hline $\mathrm{CH} 4$ & CONCENTRATION PPM & \\
\hline NMAC & CONCENTRATION PPM & \\
\hline THC & MASS GRAMS & \\
\hline co & MASS GRAMS & \\
\hline $\mathrm{CO} 2$ & MASS GRAMS & \\
\hline NOX & MASS GRAMS & 、 \\
\hline CH4 & MASS GRAMS & \\
\hline NMHC & MASS GRAMS (FID) & \\
\hline UEI & MASS KG & \\
\hline
\end{tabular}

FUEL ECONOMY MPG (L/100KM)

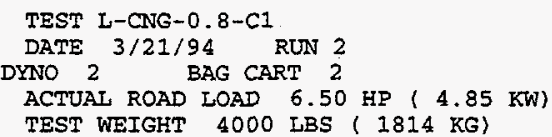

DRY BULB TEMPERATURE $78.0^{\circ} \mathrm{F}\left(25.6^{\circ} \mathrm{C}\right)$

$$
\begin{gathered}
1 \\
\text { COLD TRANSIENT } \\
(0-505 \text { SEC. }) \\
505.2 \\
.972) .983 \\
3.60(5.80) \\
563.6(15.96) \\
.28(1.01) \\
4748 .(134.5\}
\end{gathered}
$$

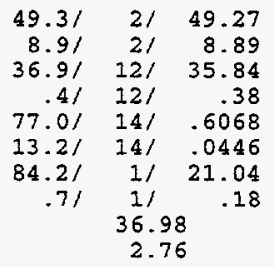

15.70

40.94

40.94
34.27

.5651

20.87

34.39

3.11

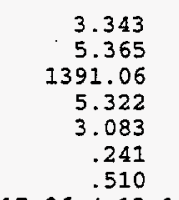

$17.96(13.10)$

3-BAG COMPOSITE RESULTS

PROJECT NO. 08-6068-001

$\begin{array}{llr}\text { THC } & \text { G/MI } & .961 \\ \text { CO } & \text { G/MI } & 1.081 \\ \text { NOX } & \text { G/MI } & 1.021\end{array}$

FUEL ECONOMY MPG (L/100KM)

$18.01(13.06)$

STABILIZED

(505-1372 SEC.) 867.3

$.975) .983$

$3.83(6.16)$

$565.9(16.03)$

$28(.01)$

8185. (231.8)

$\begin{array}{rrr}35.9 / & 2 / & 35.88 \\ 8.3 / & 2 / & 8.30 \\ 13.1 / & 12 / & 12.58 \\ .0 / & 12 / & .00 \\ 63.5 / & 14 / & .4145 \\ 13.1 / & 14 / & .0442 \\ 22.6 / & 1 / & 5.72 \\ .7 / & 1 / & .18 \\ & 27.67 & \\ & 2.72 & \end{array}$

23.03
27.94

27.94
12.22

.3722

5.55

25.07

.07
.37

3.927

3.299

1579.67

2.440

3. 874

.049

$16.85(13.96)$

CNG

FUEL DENSTTY $5.601 \mathrm{IB} / \mathrm{GAI}$

$\mathrm{H} .235 \quad \mathrm{C} .720 \quad 0.000 \quad \mathrm{X} .045$

NOX HUMIDITY C.F. $\quad .992$

3

HOT TRANSIENT

( $0-505$ SEC. )

505.2

$.973 / .983$

$3.62(5.83)$

$563.1(15.95)$

$.27(.01)$

4743. (134.3)

$45.6 / 2 / \quad 45.57$

$7.4 / 2 / 7.40$

$\begin{array}{lll}29.3 / & 12 / & 28.38\end{array}$

$\begin{array}{lll}.1 / & 12 / & .09\end{array}$

$\begin{array}{llll}72.5 / & 14 / & .5357\end{array}$

$12.9 / 14 / \quad 0434$

$79.9 / \quad 1 / 19.98$

$.7 / 1 / \quad .18$

34.81

2.51

17.79

38.59

38.59
27.39

.4948

19.81

32.44

2.91

3.140

1216.83

5.046

5.046
2.905

.225

.446

$20.63(11.40)$ 
SOUTHWEST RESEARCH INSTITUTE - DEPARTMENT OF EMISSIONS RESEARCH 3-BAG CARB FTP VEHICLE EMISSION RESULTS

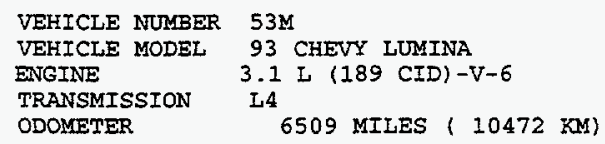

BAROMETER 29.22 IN HG (742.2 MM HG) RELATIVE HUMIDITY 25.0 PCT.

BAG NUMBER

BAG DESCRIPTION

RUN TIME SECONDS

DRY/WET CORRECTION FACTOR, SAMP/BACK

MEASURED DISTANCE MILES (KM)

BLOWER FLOW RATE SCFM (SCMM)

GAS METER FLOW RATE SCFM (SCMM)

TOTAL FLOW SCF (SCM)

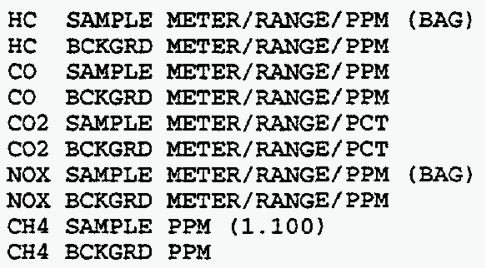

CH4 BCKGRD PPM

\section{DILUTION FACTOR}

HC CONCENTRATION PPM

CO CONCENTRATION POM

$\mathrm{CO} 2$ CONCENTRATION PCT

NOX CONCENTRATION PPM

CH4 CONCENTRATION PPM

NMHC CONCENTRATION PPM

\section{THC MASS GRAMS \\ CO MASS GRAMS \\ $\mathrm{CO} 2$ MASS GRAMS \\ NOX MASS GRAMS \\ CH4 MASS GRAMS \\ NMHC MASS GRAMS (FID) \\ FUEL MASS KG}

FUEL ECONOMY MPG (L/10OKM)

3-BAG COMPOSITE RESULTS

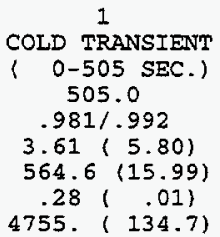

(D)

$\begin{array}{rcr}31.5 / & 2 / & 31.51 \\ .2 / & 2 / & .20\end{array}$

38.51

16.08

43.85

33.90

.5516

31.33

36.16

4.07

3.607

5.313

1359.84

6.738

3.246

.316

$18.37(12.81)$

$17.42 ; 13.51)$

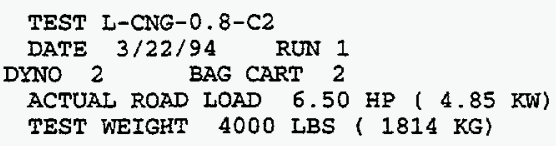

DRY BULB TEMPERATURE $75.0^{\circ} \mathrm{F}\left(23.9^{\circ} \mathrm{C}\right)$

$$
\begin{aligned}
& \stackrel{2}{2} \text { STABILIZED } \\
& \text { (505-1372 SEC.) } \\
& 867.3 \\
& .985 / .992 \\
& 3.86(6.22) \\
& 566.8(16.05) \\
& .28(.01) \\
& 8197 .(232.1) \\
& 39.6 / \\
& 9.6 / 2 / 39.58 \\
& 8.2 / 2 / \quad 8.20 \\
& 2.3 / 12 / 11.80 \\
& .3 / \quad 12 / \quad .28 \\
& 62.7 / 14 / .4049 \\
& 13.21 \quad 14 / .0446 \\
& \begin{array}{rrr}
13.2 / & 14 / & .0446 \\
32.9 / & 1 / & 8.29
\end{array} \\
& \begin{array}{lll}
1.2 / 1 / & 1 / 31
\end{array} \\
& 2.61
\end{aligned}
$$

PROJECT NO. 08-6068-001

CNG

FUEL DENSITY $5.601 \mathrm{LB} / \mathrm{GAL}$

$\begin{array}{llllll}\text { H. } & .235 & \mathrm{C} .720 & 0.000 & \mathrm{X} .045\end{array}$

NOX HUMIDITY C.F. .835

3

HOT TRANSIENT

( 0 - 505 SEC.)

505.4

$.982 / .992$

$3.61(5.81)$

$563.8(15.97)$

$.27(.01)$

4751. ( 134.6$)$

$50.7 / 2 / 50.67$

$8.1 / 21 \quad 8.10$

$46.9 / 12 / 45.69$

$.5 / \quad 12 / \quad .47$

$71.9 / \quad 14 / .5268$

$13.5 / 14 / 0458$

$24.9 / 2124.91$

.2121

38.51

18.01

43.02

44.17

.4836

24.72

35.92

3.51

3.526

6.919

1191.36

5.314

3.222

.273

$20.91 ;(11.25)$

$\begin{array}{llr}\text { CH4 } & \text { G/MI } & 1.008 \\ \text { NMHC } & \text { G/MI } & .060 \\ \text { CARBONYL G/MI } & .003 \\ \text { ALCOHOL } & \text { G/MI } & .009\end{array}$

.060
.003
.009

$\begin{array}{lll}\text { THC } & \text { G/MI } & 1.079 \\ \text { CO } & \text { G/MI } & 1.242 \\ \text { NOX } & \text { G/MI } & 1.190\end{array}$

FUEL ECONOMY MPG (L/100kM)

$18.48(12.73)$ 
COMPUTER PROGRAM IDT 1.5-R

VEHICLE NUMBER $53 \mathrm{M}$

VEHICLE MODEI 93 CHEVY IUMINA

ENGINE

3.1 L (189 CID) $-\mathrm{V}-6$

6327 MIEES ( $10180 \mathrm{KM}$ )

ODOMETER

BAROMETER 29.22 IN HG (742.2 MM HG)

RELATIVE HUMIDITY 66.6 PCT.

BAG NUMBER

BAG DESCRIPTION

RUN TIME SECONDS

DRY/WET CORRECTION FACTOR, SAMP/BACX

MEASURED DISTANCE MILES (KM)

BLOWER FLOW RATE SCFM (SCMM)

GAS METER FLOW RATE SCEM (SCMM)

TOTAL FLOW SCF (SCM)

HC SAMPLE METER/RANGE/PPM (BAG)

HC BCKGRD METER/RANGE/PPM

CO SAMPLE METER/RANGE/PPM

CO BCKGRD METER/RANGE/PPM

CO2 SAMPLE METER/RANGE/PCT

CO2 BCRGRD METER/RANGE/PCT

NOX SAMPLE METER/RANGE/PPM (BAG) (D)

NOX BCKGRD METER/RANGE/PPM

CH4 SAMPLE PPM (1.110)

CH4 BCKGRD PPM

DILUTION FACTOR

HC CONCENTRATION PPM

CO CONCENTRATION PPM

$\mathrm{CO} 2$ CONCENTRATION PCT

NOX CONCENTRATION PPM

CH4 CONCENTRATION PPM

NMHC CONCENTRATION PPM

THC MASS GRAMS

CO MASS GRAMS

$\mathrm{CO} 2$ MASS GRAMS

NOX MASS GRAMS

CH4 MASS GRAMS

NMHC MASS GRAMS (FID)

FUEU MASS KG

FUEL ECONOMY MPG (L/100KM)

3-BAG COMPOSITE RESULTS

$\begin{array}{llr}\text { THC } & \text { G/MI } & 3.123 \\ \text { CO } & \text { G/MI } & 14.839 \\ \text { NOX } & \text { G/MI } & 1.199\end{array}$

FUEL ECONOMY MPG (L/100KM)

$17.52(13.43)$
TEST L-CNG-1.0-E2 DATE $3 / 7 / 94$ RUN 1 DYNO 2 BAG CART 2

ACTUAI ROAD LOAD $6.50 \mathrm{HP}(4.85 \mathrm{KW})$ TEST WEIGHT 4000 LBS ( $1814 \mathrm{KG}$ )

DRY BULB TEMPERATURE $76.0^{\circ} \mathrm{F}\left(24.4^{\circ} \mathrm{C}\right)$

$$
\begin{gathered}
1 \\
\text { COLD TRANSIENT } \\
(0-505 \text { SEC. } \\
505.2 \\
.968) .979 \\
3.63(5.84) \\
564.5(15.99) \\
.27(.01) \\
4755 .(134.7)
\end{gathered}
$$$$
\begin{array}{rrr}
90.2 / & 2 / & 90.15 \\
10.0 / & 2 / & 9.99 \\
55.0 / & 1 / & 504.72 \\
.2 / & 1 / & 1.68 \\
75.6 / & 14 / & .5838 \\
13.9 / & 14 / & .0474 \\
90.1 / & 1 / & 22.48 \\
1.3 / & 1 / & .34 \\
& 62.45 & \\
& 2.73
\end{array}
$$$$
15.09
$$

15.09
80.82

483.66

.5396

22.17

59.90

14.33

6.682

75.825

1330.36

6.208

5.377

1. 113

$17.29 ; 13.61)$

$$
\begin{gathered}
2 \\
\text { STABIIIZED } \\
(505-1372 \text { SEC.) } \\
867.5 \\
.971 / .979 \\
3.85(6.20) \\
564.2(15.98) \\
.27(.01) \\
8161 .(231.1)
\end{gathered}
$$

$\begin{array}{rrr}11.5 / & 3 / & 114.74 \\ 1.2 / & 3 / & 11.97 \\ 74.6 / & 13 / & 178.42 \\ .7 / & 13 / & 1.52 \\ 62.4 / & 14 / & .4013 \\ 13.7 / & 14 / & .0466 \\ 29.1 / & 1 / & 7.35 \\ 1.7 / & 1 / & .44 \\ & 83.08 & \\ & 2.94 & \end{array}$

22.52

103.30

171.05

.3568

6.93

80.27

14.21

14.603

46.026

46.026
1509.87

3.329

12.368

1.893

.587

$16.60(14.17)$

CH4 G/MI

NMHC

G/MI

CARBONYL G/MI

ALCOHOL G/MI
ESEARCH

PROJECT NO. 08-6068-001

CNG

FUEL DENSITY $5.573 \mathrm{LB} / \mathrm{GAL}$

H $.236 \quad$ C $.725 \quad 0.000 \quad \times .040$

NOX HUMIDITY C.F. 1.087

3

HOT TRANSIENT

( $0-505$ SEC. )

505.0

$.969 / .979$

$3.62(5.83)$

$564.5(15.99)$

$.27(.01)$

4753. (134.6)

$13.6 / 3 / 135.70$

$1.1 / 3 / 10.98$

$42.7 /$ I/ 378.16

$.3 /$ 1/ 2.52

$71.3 / 14 / \quad .5181$

$13.8 / 14 / .0470$

$75.3 /$ i/ 18.84

$1.01 \quad 1 / \quad .26$

99.70

17.05

125.37

361.95

.4738

18.60

96.94

17.76

10.311

56.723

1167.81

5.207

8.700

1.378

$19.71(13.94)$

2.626
.422
.075
.000 
COMPUTER PROGRAM LDT $1.5-\mathrm{R}$

VEHICLE NUMBER $53 \mathrm{M}$

VEHICLE MODEL 93 CHEVY LUMINA

ENGINE

3.1 I $(189$ CID $\rangle-\mathrm{V}-6$

TRANSMISSION I4

ODOMETER 6275 MILES ( $10096 \mathrm{KM}$ )

BAROMETER 28.95 IN HG (735.3 MM HG)

REIATIVE HUMIDITY $59.7 \mathrm{PCT}$.

BAG NUMBER

BAG DESCRIPTION

RUN TIME SECONDS

DRY/WET CORRECTION FACTOR, SAMP/BACK

MEASURED DISTANCE MILES (KM)

BLOWER FLOW RATE SCFM (SCMM)

GAS METER FLOW RATE SCFM (SCMM)

TOTAL FLOW SCF (SCM)

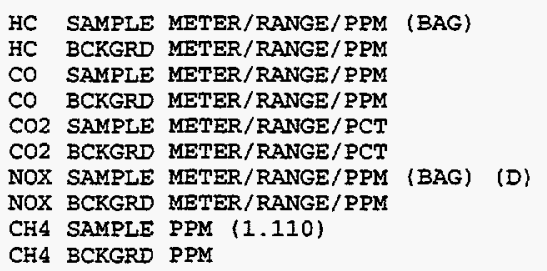

CHA BCKGRD PRM

DIIUTION FACTOR

HC CONCENTRATION PPM

CO CONCENTRATION PPM

$\mathrm{CO} 2$ CONCENTRATION PCT

NOX CONCENTRATION PPM

CH4 CONCENTRATION PPM

NMHC CONCENTRATION PPM

THC MASS GRAMS

CO MASS GRAMS

$\mathrm{CO} 2$ MASS GRAMS

NOX MASS GRAMS

CH4 MASS GRAMS

NMHC MASS GRAMS (FID)

FUEI MASS KG

FUEL ECONOMY MPG (L/IOOKM)

(BAG)
(BAG) (D)

$$
\text { 3-BAG CARB FTP VEHICLE EMISSION RESULTS }
$$

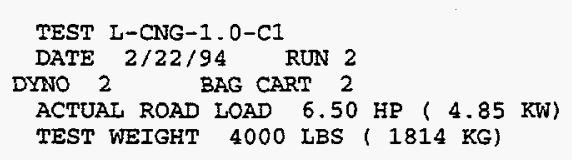

DRY BULB TEMPERATURE $77.0^{\circ} \mathrm{F}\left(25.0^{\circ} \mathrm{C}\right)$

$$
\begin{gathered}
1 \\
\text { COLD TRANSIENT } \\
(0-505 \text { SEC. }) \\
504.6 \\
.969 / .980 \\
3.64(5.86) \\
561.7(15.91) \\
.27(1.01) \\
4726 .(133.8)
\end{gathered}
$$

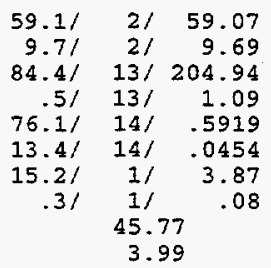

3.99

$$
\begin{array}{r}
15.66 \\
49.99 \\
196.42 \\
.5495 \\
3.79 \\
42.04 \\
3.33 \\
4.033 \\
30.604 \\
1346.38 \\
1.024 \\
3.751 \\
.257 \\
.508
\end{array}
$$$$
18.09(13.00)
$$

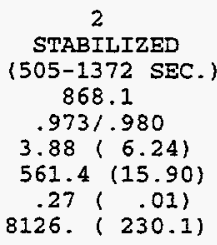

$27.3 / 2 / 27.28$

$\begin{array}{rrr}27.37 & 27 & 27.28 \\ 9.7 / & 21 & 9.69\end{array}$

$\begin{array}{rrr}17.7 / & 12 / & 17.05\end{array}$

$1.1 / 12 / 1.04$

$63.0 / 14 / 4085$

$13.5 / 14 / .0458$

$6.2 / 1 / 1.59$

$.3 / 1 / \quad .08$

19.62
3.62

$$
\begin{array}{r}
23.42 \\
18.00 \\
15.53 \\
.3646 \\
1.52 \\
16.16
\end{array}
$$

2. 521

4.162

1536.36

.703

2. 479

.009

$17.49\lceil 13.45\rangle$

$\begin{array}{lll}\text { CH4 } & \text { G/MI } & .839 \\ \text { NMHC } & \text { G/MI } & .034 \\ \text { CARBONYL } & \text { G/MI } & .003 \\ \text { ALCOHOL } & \text { G/MI } & .007\end{array}$

3-BAG COMPOSITE RESULTS

$\begin{array}{llr}\text { THC } & \text { G/MI } & .882 \\ \text { CO } & \text { G/MI } & 3.690 \\ \text { NOX } & \text { G/MI } & .201\end{array}$

FUEL ECONOMY MFG (I/100kM)
PROJECT NO. 08-6068-001
CNG

LENSITY $5.573 \mathrm{LB} / \mathrm{GAL}$

NOX HUMIDITY C.F. 1.055

$$
\begin{gathered}
3 \\
\text { HOT TRANSIENT } \\
(0-505 \text { SEC. }) \\
505.8 \\
.970 / .980 \\
3.64(5.86) \\
552.6(15.65) \\
.26(1.01) \\
4661 .(132.0)
\end{gathered}
$$

$61.2 / 2 / \quad 61.16$

$9.6 / 21 \quad 9.59$

$53.8 / 13 / 124.77$

$.7 / 13 / 1.52$

$\begin{array}{lll}73.0 / & 14 / & .5432\end{array}$

$14.1 / 14 / .0482$

$9.5 / 1 / 2.43$

$.1 / 1 / \quad .03$

47.71
3.73

17.23

52.13

118.96

.4978

2.40

44.20

3.06

4.170

18.280

1203.07

.640

3.889

.233

$20.46(11.50)$

$18.36(12.81)$ 
COMPUTER PROGRAM LDT $1.5-R$

SOUTHWEST RESEARCH INSTITUTE - DEPARTMENT OF EMISSIONS RESEARCH

3-BAG CARB FTP VEHICIE EMISSION RESULTS

VEHICLE NUMBER $53 \mathrm{M}$

VEHICLE MODEL 93 CHEVY IUMINA

ENGINE

3.1 I $(189$ CID $)-V-6$

L4

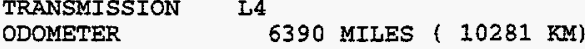

BAROMETER 29.60 IN HG (751.8 MM HG)

RELATIVE HUMIDITY 23.8 PCT.

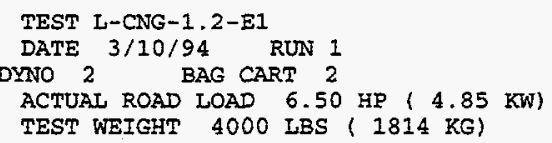

DRY BULB TEMPERATURE $71.0^{\circ} \mathrm{F}\left(21.7^{\circ} \mathrm{C}\right)$
BAG NUMBER

BAG DESCRIPTION

RUN TIME SECONDS

DRY/WET CORRECTION FACTOR, SAMP/BACK

MEASURED DISTANCE MILES (KM)

BLOWER FLOW RATE SCFM (SCMM)

GAS METER FLOW RATE SCFM (SCMM)

TOTAL FLOW SCF (SCM)

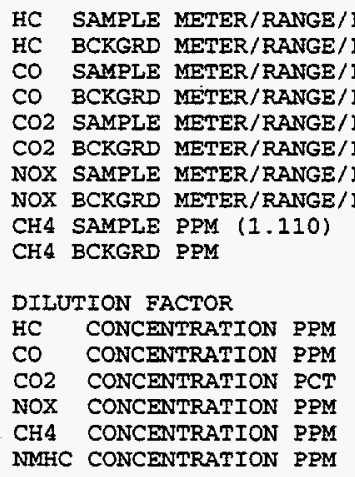

THC MASS GRAMS

CO MASS GRAMS

$\mathrm{CO} 2$ MASS GRAMS

NOX MASS GRAMS

CH4 MASS GRAMS

NMFC MASS GRAMS (EID)

FUEL MASS KG

FUEL ECONOMY MPG (L/100KM)

(BAG) PT $\begin{array}{lll}71.2 / & 14 / & .5166 \\ 13.3 / & 14 / & .0450\end{array}$ (BAG) (D) $\begin{array}{ccc}13.3 / & 14 / & .0450\end{array}$

$\begin{array}{rrr}30.6 / & 1 / & 7.72 \\ 1.5 / & 1 / & .39\end{array}$ $1 /$
495.81
3.46

$$
\begin{gathered}
1 \\
\text { COLD TRANSIENT } \\
(0-505 \text { SEC. }) \\
505.7 \\
.984 / .994 \\
3.62(5.83) \\
573.7(16.25) \\
.28(.01) \\
4837 .(137.0)
\end{gathered}
$$

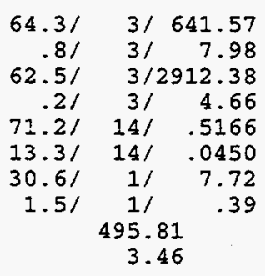

11.24

11.24
634.30

2841.53

.4756

.475
7.37

492.66

87.44

52.543

453.180

1192.92

1.575

44.993

6.907

$12.37(19.02)$

3-BAG COMPOSITE RESULTS

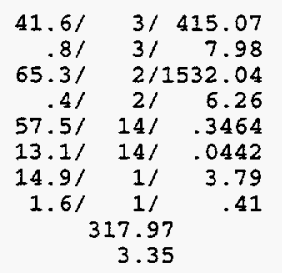

18.07

18.07
407.53

1498.78

.3046

3.40

314.80

58.10

58.203

412.317

1317.87

1.255

49.593

4.917
.767

.767

$12.76(18.43)$

CH4 $\quad \mathrm{G} / \mathrm{MI}$

NMHC G/MI

CARBONYL G/MI

ALCOHOL G/MI
PROJECT NO. 08-6068-001
CNG

FUEL DENSITY $5.573 \mathrm{LB} / \mathrm{GAI}$

H $.236 \quad \mathrm{C} .725 \quad 0.000 \quad \mathrm{X} .040$

NOX HUMIDITY C.F. $\quad .816$

$$
3
$$

HOT TRANSIENT

( 0- 505 SEC.)

505.4

$.985 / .994$

$3.63(5.83)$

$571.6(16.19)$

$.28(.01)$

4817. (136.4)

$\begin{array}{rrr}29.8 / & 3 / & 297.34 \\ .8 / & 3 / & 7.98 \\ 77.8 / & 2 / 2006.86 \\ .5 / & 2 / & 7.83 \\ 67.2 / & 14 / & .4613 \\ 12.9 / & 14 / & .0434 \\ 31.0 / & 1 / & 7.82 \\ 1.2 / & 1 / & .31 \\ 225.07 & \\ 3.64 & \end{array}$

14.11

289.92
1956.98

.4210

7.53

221.69

43.84

\begin{tabular}{|c|c|c|c|c|c|}
\hline THC & G/MI & 12.596 & $\mathrm{CH} 4$ & G/MI & 10.728 \\
\hline $\mathrm{CO}$ & G/MI & 104.618 & NMHC & $\mathrm{G} / \mathrm{MI}$ & 1.714 \\
\hline Nox & $\mathrm{G} / \mathrm{MI}$ & .380 & $\begin{array}{l}\text { CARBONYL } \\
\text { ALCOHOL }\end{array}$ & $\begin{array}{l}\mathrm{G} / \mathrm{MI} \\
\mathrm{G} / \mathrm{MI}\end{array}$ & $\begin{array}{l}.151 \\
.001\end{array}$ \\
\hline
\end{tabular}

23.921

310.825

1051.54

1. 604

20.163

3. 449

$15.80(14.89)$ 
SOUTHWEST RESEARCH INSTITUTE - DEPARTMENT OF EMISSIONS RESEARCH

3-BAG CARB FTP VELICLE EMISSION RESULTS

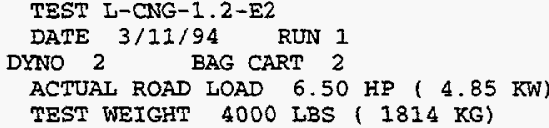

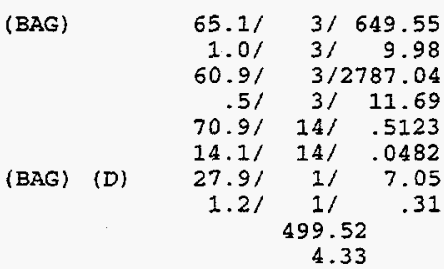

52.905

430.986

1172.54

1.489

45.182

7.126

$12.71 ; 18.51$ $71.0^{\circ} \mathrm{F}\left(21.7^{\circ} \mathrm{C}\right)$

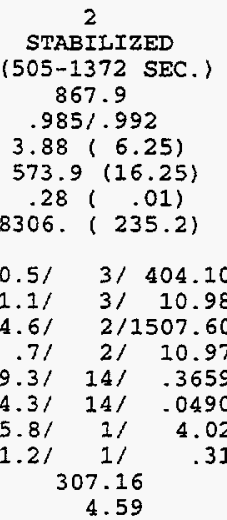

17.56

393.75

1466.24

.3197

3.73

302.83

57.61

56.311

401.532

1376.67

1.411

47.490

7.814

.781

$12.57(18.71)$

$\mathrm{CH} 4$

NMHC

G/MI

$G / M I$

COHOI G/MI
PROJECT NO. 08-6068-001

CNG

FUEL DENSITY $5.573 \mathrm{LB} / \mathrm{GAI}$

$\mathrm{H} .236 \mathrm{C} .7250 .000 \mathrm{X} .040$

NOX HUMIDITY C.F. $\quad .842$

\section{3}

HOT TRANSIENT

( $0-505$ SEC.)

505.3

$983 / .992$

$3.59(5.77)$

$570.8(16.17)$

$27(.01)$

4810 . ( 136.2$)$

$\begin{array}{rrr}28.0 / & 3 / 279.38 \\ 1.0 / & 3 / 2 & 9.98\end{array}$

$\begin{array}{rrr}1.0 / & 3 / 29.98 \\ 77.6 / & 2 / 1998.68\end{array}$

$8 / 2 / 2.55$

$67.2 / \quad 14 / \quad .4613$

$14.4 / 14 / \quad .0494$

$32.3 /$ if 8.14

$1.4 / \quad 1 / \quad 36$

208.32

4.13

14.17

270.10

1940.27

.4154

7.81

204.48

43.13

22.292

307.669

1035.88

1.711

18.568

3.387

.571

$15.87(14.82)$

$\begin{array}{llr}\text { THC } & \text { G/MI } & 12.256 \\ \text { CO } & \text { G/MI } & 101.816 \\ \text { NOX } & \text { G/MI } & .404\end{array}$

FUEL ECONOMY MPG (L/100KM) 13.40 (17.56)

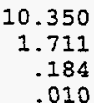


SOUTHWEST RESEARCH INSTITUTE - DEPARTMENT OF EMISSIONS RESEARCH

3-BAG CARB FTP VEHICLE EMISSION RESULTS

PROJECT NO. 08-6068-001

VEHICLE NUMBER $53 \mathrm{M}$

VEHICLE MODEL 93 CHEVY LUMINA

ENGINE

3.1 I (189 CID) $-\mathrm{V}-6$

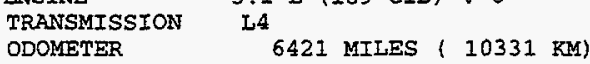

RELATIVE HUMIDITY 65.8 PCT.

BAG NUMBER

BAG DESCRIPTION

RUN TIME SECONDS

MEASURED DISTANCE MILES (KM)

BLOWER FLOW RATE SCFM (SCMM)

GAS METER FLOW RATE SCFM (SCMM)

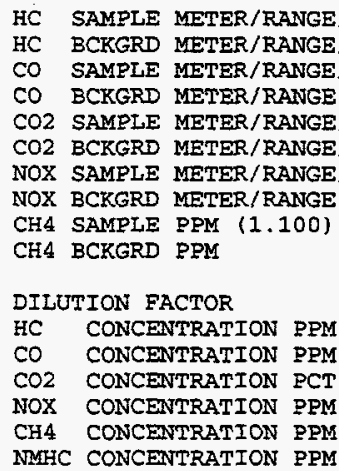

THC MASS GRAMS

CO MASS GRAMS

$\mathrm{CO} 2$ MASS GRAMS

NOX MASS GRAMS

$\mathrm{CH} 4$ MASS GRAMS

NMHC MASS GRAMS (FID)

FUEI MASS KG

FUEL ECONOMY MPG (L/100kM)

\section{(BAG)} . (BAG) (D)
BAROMETER 29.19 IN HG (741.4 MM HG)

DRY/WET CORRECTION FACTOR, SAMP/BACK

TOTAL ELOW SCF (SCM)

TEST WEIGHT 4000 LBS $(1814 \mathrm{KG})$
DRY BULB TEMPERATURE $74.0^{\circ} \mathrm{F}\left(23.3^{\circ} \mathrm{C}\right)$

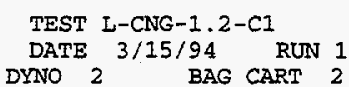

3-BAG COMPOSITE RESULTS

\begin{tabular}{|c|c|c|}
\hline $\begin{array}{l}\mathrm{THC} \\
\mathrm{CO}\end{array}$ & $\begin{array}{l}G / M I \\
G / M I\end{array}$ & $\begin{array}{r}9.129 \\
39.053\end{array}$ \\
\hline NOX & G/MI & .016 \\
\hline
\end{tabular}

CH4

G/MI

NMFC G/MI

CARBONYL G/MI ALCOHOL G/MI 
SOUTHWEST RESEARCH INSTITUTE - DEPARTMENT OF EMISSIONS RESEARCH

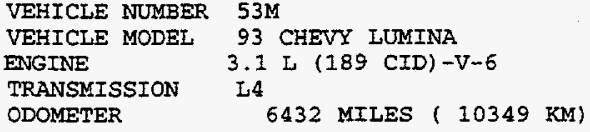

PAROMETER 29.22 IN HG (742.2 MM HG) RELATIVE HUMIDITY 72.8 PCT.

BAG NUMBER

BAG DESCRIPTION

RUN TIME SECONDS

DRY/WET CORRECTION FACTOR, SAMP/BACR

MEASURED DISTANCE MILES (RM)

BLOWER FLOW RATE SCFM (SCMM)

GAS METER FLOW RATE SCFM (SCMM)

TOTAL FLOW SCE (SCM)

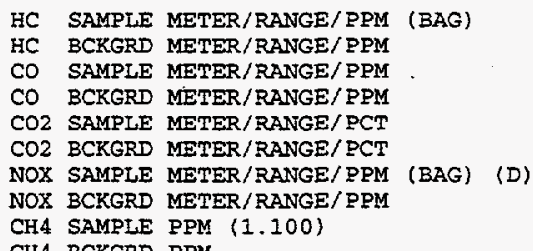

CH4 SAMPLE PPM $(1.100)$

CH4 BCKGRD PPM

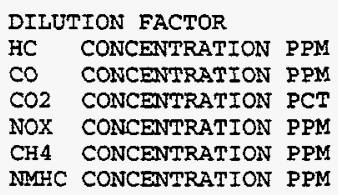

$\begin{array}{ll}\text { THC } & \text { MASS GRAMS } \\ \text { CO } & \text { MASS GRAMS } \\ \text { CO2 } & \text { MASS GRAMS } \\ \text { NOX } & \text { MASS GRAMS } \\ \text { CH4 } & \text { MASS GRAMS } \\ \text { NMHC } & \text { MASS GRAMS (FID) } \\ \text { FUEL } & \text { MASS KG }\end{array}$

FUEL ECONOMY MPG (L/100KM)

3-BAG COMPOSITE RESULTS
3-BAG CARB FTP VEHICLE EMISSION RESULTS

$$
\begin{aligned}
& \text { TEST L-CNG- } 1.2-C 2 \\
& \text { DATE } 3 / 16 / 94 \text { RUN } 1 \\
& \text { DYNO } 2 \text { BAG CART } 2 \\
& \text { ACIUAL ROAD LOAD } 6.50 \mathrm{HP}(4.85 \mathrm{KW}) \\
& \text { TEST WEIGET 4000 LBS ( } 1814 \mathrm{KG})
\end{aligned}
$$

DRY BULB TEMPERATURE $71.0^{\circ} \mathrm{F}\left(21.7^{\circ} \mathrm{C}\right)$

$$
\begin{aligned}
& \text { COLD TRANSIENT } \\
& \text { (0-505 SEC.) } \\
& 506.1 \\
& .967 / .981 \\
& 3.63(5.84) \\
& 564.8(16.00) \\
& 27(.01) \\
& \text { 4766. ( } 135.0)
\end{aligned}
$$

46.937

255.622

1617.21

.088

44.396

2.497

$11.83(19.89)$

$$
\begin{aligned}
& 2 \\
& \text { STABILIZED } \\
& \text { (505-1372 SEC.) } \\
& 867.2 \\
& 971.981 \\
& 3.90(6.28) \\
& 564.7(15.99) \\
& .28(.01) \\
& 8166 \text {. (231.3) } \\
& 30.5 / 3 / 304.32 \\
& 1.3 / 3 / 12.97 \\
& 54.4 / \quad 1 / 498.28 \\
& .4 / 1 / 3.35 \\
& 69.4 / 14 / .4911 \\
& 13.0 / 14 / .0438 \\
& \begin{array}{rrr}
1.1 / & 1 / & .28 \\
.7 / & 1 / & .18
\end{array} \\
& 259.92 \\
& 3.73 \\
& 17.05 \\
& 292.11 \\
& 476.29 \\
& .4499 \\
& \begin{array}{r}
.11 \\
256.41
\end{array} \\
& 10.06
\end{aligned}
$$

PROJECT NO. 08-6068-001

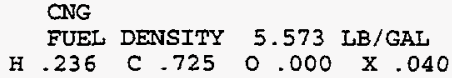

NOX HUMIDITY C.F. 1.049

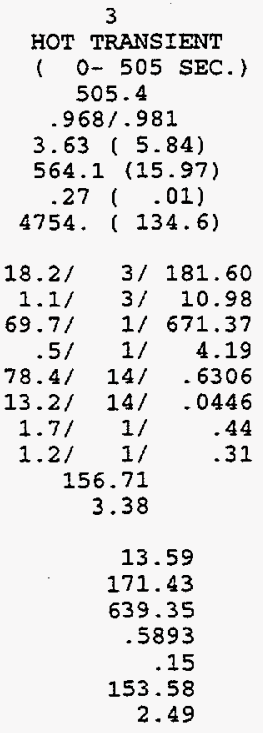

13.984

100.203

1452.47

.041

13.784

.193

.596

$15.38(15.29)$

$\begin{array}{llr}\text { CH4 } & \text { G/MI } & 8.829 \\ \text { NMHC } & \text { G/MI } & .335 \\ \text { CARBONYL } & \text { G/MI } & .004 \\ \text { ALCOHOL } & \text { G/MI } & .000\end{array}$




\section{APPENDIX E}

\section{COMPUTER PRINTOUTS OF EMISSIONS DATA WITH REFORMULATED GASOLINE}

\begin{tabular}{|c|l|l|l||}
\hline Page E- & \multicolumn{1}{|c|}{ Test Number } & $\begin{array}{l}\text { Operating } \\
\text { Condition }\end{array}$ & \multicolumn{1}{|c|}{$\begin{array}{c}\text { Catalyst } \\
\text { Installation }\end{array}$} \\
\hline \hline 1 & L-PH2-0.8-E1 & Lean & Without Catalyst \\
\hline 2 & L-PH2-0.8-E2 & Lean & Without Catalyst \\
\hline 3 & L-PH2-0.8-C1 & Lean & With Catalyst \\
\hline 4 & L-PH2-0.8-C2 & Lean & With Catalyst \\
\hline \hline 5 & L-PH2-1.0-E1 & Stoich & Without Catalyst \\
\hline 6 & L-PH2-1.0-E2 & Stoich & Without Catalyst \\
\hline 7 & L-PH2-1.0-C1 & Stoich & With Catalyst \\
\hline 8 & L-PH2-1.0-C2 & Stoich & With Catalyst \\
\hline \hline 9 & L-PH2-1.2-E1 & Rich & Without Catalyst \\
\hline 10 & L-PH2-1.2-E2 & Rich & Without Catalyst \\
\hline 11 & L-PH2-1.2-C1 & Rich & With Catalyst \\
\hline 12 & L-PH2-1.2-C2 & Rich & With Catalyst \\
\hline
\end{tabular}


SOUTHWEST RESEARCH INSTITUTE - DEPARTMENT OF EMISSIONS RESEARCH

COMPUTER PROGRAM LDT $1.5-R$
3-BAG CARB FTP VEHICLE EMISSION RESULTS

$$
\begin{aligned}
& \text { TEST L-PH2-0.8-EI } \\
& \text { DATE } 4 / 22 / 94 \text { RUN } 1 \\
& \text { DYNO } 2 \quad \text { BAG CART } 2 \\
& \text { ACTUAL ROAD LOAD } 6.50 \text { HP }(4.85 \mathrm{KW}) \\
& \text { TEST WEIGHT } 4000 \text { LBS }(1814 \mathrm{kG})
\end{aligned}
$$

DRY BULB TEMPERATURE $76.0^{\circ} \mathrm{F}\left(24.4^{\circ} \mathrm{C}\right\}$

$$
\begin{aligned}
& \text { I } \\
& \text { COLD TRANSIENT } \\
& (0-505 \text { SEC. } \\
& 506.0 \\
& .974 / .981 \\
& 3.60(5.80) \\
& 559.3(15.84) \\
& .27(51) \\
& 4719 .(133.6)
\end{aligned}
$$

GAS METER FLOW RATE SCFM (SCMM)

TOTAL FLOW SCF (SCM)

HC SAMPLE METER/RANGE/PPM (BAG)

HC BCKGRD METER/RANGE/PRM

CO SAMPLE METER/RANGE/PPM

CO BCRGRD METER/RANGE/PPM

CO2 SAMPLE METER/RANGE/PCT

CO2 BCKGRD METER/RANGE/PCT

NOX SAMPLE METER/RANGE/PPM (BAG) (D)

NOX BCKGRD METER/RANGE/PPM

CEL4 SAMPLE PPM $(1.120)$

CE4 SCKGRD PPM

DILUTION FACTOR

HC CONCENTRATION PPM

CO CONCENTRATION PPM

$\mathrm{CO} 2$ CONCENTRATION PCT

NOX CONCENTRATION PPM

CH4 CONCENTRATION PFM

NMHC CONCENTRATION PPM

THC MASS GRAMS

CO MASS GRAMS

$\mathrm{CO} 2$ MASS GRAMS

NOX MASS GRAMS

CH4 MASS GRAMS

NMHC MASS GRAMS (FID)

FUEL MASS KG

FUEL ECONOMY MPG (L/100kM)

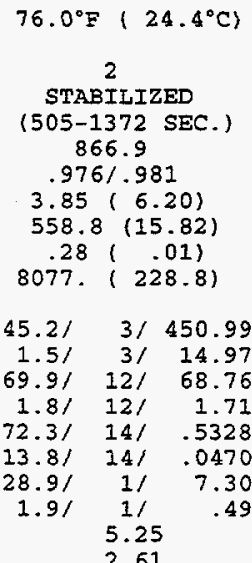

2.61

22.62

436.69

65.12

.4878

6.83

2.76

433.60

61.993

17.342

2043.17

3.084

.421

57.194

734

14.72 ( 15.98$)$
28.829

$15.89 ;(14.81)$

PROJECT NO. 08-6068-001

GASOLINE PHASE II EM-1701-F

FUEI DENSITY 6.186 LB/GAI.

H .139 C . $841 \quad 0.020 \times .000$

NOX HUMIDITY C.F. 1.032

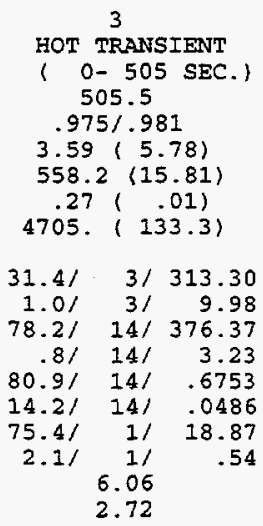

17.79

303.88

361.14

.6295

18.36

3.49

299.97

25.493

56.026

1535.69

4.830

.310

23.049

553

18.24 ( 12.90$)$

3-BAG COMPOSITE RESULTS

$\begin{array}{llr}\text { THC } & \text { G/MI } & 12.104 \\ \text { CO } & \text { G/MI } & 9.030 \\ \text { NOX } & \text { G/MI } & 1.185\end{array}$

FUEL ECONOMY MPG (I/ $/ 100 \mathrm{KM}$ )

$15.82(14.87)$

$\begin{array}{llr}\text { CH4 } & \text { G/MI } & .101 \\ \text { NMHC } & \text { G/MI } & 11.106 \\ \text { CARBONII } & \text { G/MI } & .760 \\ \text { ALCOHOI } & \text { G/MI } & .136\end{array}$


SOUTHWEST RESEARCH INSTITUTE - DEPARTMENT OF EMISSIONS RESEARCH

3-BAG CARB FTP VEHICLE EMISSION RESULTS

PROJECT NO. 08-6068-001

VEHICLE NUMBER $53 \mathrm{M}$

VEHICLE MODEL 93 CHEVY LUMINA

ENGINE

3.1 I (189 CID) $-V-6$

TRANSMISSION L4

ODOMETER

6824 MILES ( $10979 \mathrm{kM}$ )

BAROMETER 28.98 IN HG (736.1 MM HG) RET_ATIVE HUMIDITY 67.5 PCT.

BAG NUMBER

BAG DESCRIPTION

RUN TIME SECONDS

DRY/WET CORRECTION FACTOR, SAMP/BACK

MEASURED DISTANCE MILES (KM)

BLOWER FIOW RATE SCFM (SCMM)

GAS METER FLOW RATE SCFM (SCMM)

TOTAL FLOW SCF (SCM)

HC SAMPLE METER/RANGE/PPM (BAG)

HC BCKGRD METER/RANGE/PPM

CO SAMPIL METER/RANGE/PPM

CO BCKGRD METER/RANGE/PPM

CO2 SAMPLE METER/RANGE/PCT

CO2 BCKGRD METER/RANGE/PCT

NOX SAMPLE METER/RANGE/PPM (BAG) (D)

NOX BCRGRD METER/RANGE/PPM

CH4 SAMPLE PPM $(1.120)$

$\mathrm{CH} 4$ BCKGRD PPM

DILUTION FACTOR

HC CONCENTRATION PPM

CO CONCENTRATION PPM

$\mathrm{CO} 2$ CONCENTRATION PCT

NOX CONCENTRATION PPM

CH4 CONCENTRATION PPM

NMIC CONCENTRATION PPM

$\begin{array}{ll}\text { THC } & \text { MASS GRAMS } \\ \text { CO } & \text { MASS GRAMS } \\ \text { CO2 } & \text { MASS GRAMS } \\ \text { NOX } & \text { MASS GRAMS } \\ \text { CH4 } & \text { MASS GRAMS } \\ \text { NMHC } \\ \text { FUEL MASS GRAMS (FID) }\end{array}$

FUEL ECONOMY MPG (L/100kM)

3-BAG COMPOSITE RESULTS

$\begin{array}{llr}\text { THC } & \text { G/MI } & 11.443 \\ \text { CO } & \text { G/MI } & 9.510 \\ \text { NOX } & \text { G/MI } & 1.492\end{array}$

FUEL ECONOMY MPG $(\mathrm{L} / 100 \mathrm{KM})$

$15.97(14.73)$
1

COLD TRANSIENT

( 0-505 SEC.)

505.0

$.969 / .977$

$3.62(5.82)$

$554.5(15.71)$

$.27(.01)$

4670. (132.3)

$39.3 / 3 / 392.13$

$.8 / 3 / 7.98$

$68.2 / 14 / 319.35$

$.2 / 14 / \quad .81$

$86.8 / 14 / \quad .7938$

$13.2 / \quad 14 / \quad .0446$

$30.4 / 2 / \quad 30.41$

$\begin{array}{rrr}3 / & 21 & .30\end{array}$

6.73

2.39

15.31

384.67

306.61

.7521

30.13

4.50

379.63

31.924

47.209

1821.13

8.669

.397

28.951

$15.68(15.00)$

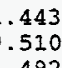

TEST I-PH2-0.8-E2

DATE $4 / 25 / 94$ RUN I

DYNO 2 BAG CART 2

ACTUAI ROAD LOAD $6.50 \mathrm{HP}(4.85 \mathrm{~kW}$ )

TEST WEIGHT 4000 LES ( $1814 \mathrm{KG}$ )

DRY BULB TEMPERATURE $78.0^{\circ} \mathrm{F}\left(25.6^{\circ} \mathrm{C}\right)$

STABILIZED

(505-1372 SEC.)

866.9

$.972 / .977$

$3.90(6.27)$

$554.4(15.70)$

$27(.01)$

8013 . (226.9)

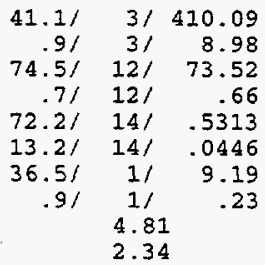

22.82

401.50

70.52

.4886

8.97

2.57

398.62

57.184

18.633

2030.23

4.426

.390

52.162
.726

$15.07(15.61)$

GASOLINE PHASE II EM-1701-F

FUEL DENSITY $6.186 \mathrm{LB} / \mathrm{GAL}$

H $.139 \quad \mathrm{C} .841 \quad 0.020 \quad \mathrm{X} .000$

NOX HUMIDITY C.F. 1.137

3

HOT TRANSIENT

( $0-505$ SEC.

505.3

9701.977

$3.61(5.81)$

$553.8(15.68)$

$.27(.01)$

4666. (132.1)

$32.8 / 3 / 327.27$

$1.1 / 3 / 10.98$

$79.8 / \quad 14 / 385.65$

$.4 / 14 / \quad 1.62$

$81.2 / 14 / .6809$

$13.5 / \quad 14 / \quad .0458$

$75.8 / \quad 1 / 18.97$

$\begin{array}{lll}1.3 / & 1 / & -34\end{array}$

5.91
2.35

17.61

316.92

370.54

.6377

18.65

3.70

312.78

26.388

57.003

1542.78

5.360

.326
23.832

.832
.556

$18.22(12.91)$

$\begin{array}{llr}\text { CH4 } & \text { G/MI } & .099 \\ \text { NMHC } & \text { G/MI } & 10.411 \\ \text { CARBONYL } & \text { G/MI } & .804 \\ \text { ALCOHOI } & \text { G/MI } & .129\end{array}$ 
COMPUTER PROGRAM LDT $1.5-R$

VEHICLE NUMBER 53M

VEHICLE MODEL 93 CHEVY IUMINA

ENGINE

3.1 I (189 CID) $-\mathrm{V}-6$ L4

TRANSMISSION

6776 MILES ( $10902 \mathrm{KM}$ )

BAROMETER 29.31 IN HG (744.5 MM HG)

REIATTVE HUMIDITY 65.3 PCT.

BAG NUMBER

BAG DESCRIPTION

RUN TIME SECONDS

DRY/WET CORRECTION FACTOR, SAMP/BACK

MEASURED DISTANCE MILES (KM)

BLOWER FLOW RATE SCFM (SCMM)

GAS METER FLOW RATE SCEM (SCMM)

TOTAL FLOW SCF (SCM)

\section{HC SAMPLE METER/RANGE/PPM (BAG) \\ HC BCKGRD METER/RANGE/PPM \\ CO SAMPLE METER/RANGE/PPM \\ CO BCKGRD METER/RANGE/PPM \\ CO2 SAMPLE METER/RANGE/PCT \\ $\mathrm{CO} 2$ BCKGRD METER/RANGE/PCT \\ NOX SAMPLE METER/RANGE/PPM (BAG) (D) \\ NOX BCKGRD METER/RANGE/PPM}

CH4 SAMPLE PPM $(1.120)$

CH4 BCKGRD PPM

DIIUTION FACTOR

HC CONCENTRATION FPM

CO CONCENTRATION PPM

$\mathrm{CO} 2$ CONCENTRATION PCT

NOX CONCENTRATION PPM

CHA CONCENTRATION PPM

NMHC CONCENTRATION PPN

$\begin{array}{lll}\text { THC } & \text { MASS GRAMS } \\ \text { CO } & \text { MASS GRAMS } \\ \text { CO2 } & \text { MASS GRAMS } \\ \text { NOX } & \text { MASS GRAMS } \\ \text { CH4 } & \text { MASS GRAMS } & \\ \text { NMHC } & \text { MASS GRAMS (FID) } \\ \text { FUEL } & \text { MASS KG } & \end{array}$

FUEI MASS KG

FUEL ECONOMY MPG (L/100KM)

3-BAG COMPOSITE RESULTS

$\begin{array}{lll}\text { THC } & \text { G/MI } & .248 \\ \text { CO } & \text { G/MI } & 4.713 \\ \text { NOX } & \text { G/MI } & 1.067\end{array}$

FUEL ECONOMY MPG (L/100KM)

\section{3-BAG COMPOSITE RESULTS}

3-BAG CARB FTP VEHICLE EMISSION RESULTS

$$
\begin{aligned}
& \text { TEST L-PH2-0.8-C1 } \\
& \text { DATE 4/20/94 RUN } 1 \\
& \text { DYNO 2 } \text { BAG CART } 2 \\
& \text { ACTUAL ROAD LOAD } 6.50 \mathrm{HP}(4.85 \mathrm{KW}) \\
& \text { TEST WEIGHT } 4000 \mathrm{LBS}(1814 \mathrm{KG})
\end{aligned}
$$

DRY BULB TEMPERATURE $73.0^{\circ} \mathrm{F}\left(22.8^{\circ} \mathrm{C}\right)$

$$
\begin{gathered}
1 \\
\text { COLD TRANSIENT } \\
(0-505 \text { SEC. }) \\
505.3 \\
.974 / .982 \\
3.61(5.81) \\
559.8(15.85) \\
.28\left(\begin{array}{c}
.01) \\
4717 .(133.6)
\end{array}\right.
\end{gathered}
$$

$47.1 / 2 / \quad 47.07$

$\begin{array}{lll}7.31 & 21 & 7.30\end{array}$

$69.2 / 14 / 324.95$

$.3 / 14 / 1.21$

$86.7 / \quad 14 / \quad .7916$

$13.4 / 14 / 0454$

$25.0 / 2 / 25.01$

$\begin{array}{lll}.3 / & 21 & .30\end{array}$

6.84

15.98

40.23

311.88

.7491

24.73

3.68

36.12

3.175

48.501

1831.92

6.508

.327

2.782

.623

$16.26(14.46)$

$$
\begin{aligned}
& 2 \\
& \text { STABILIZED } \\
& \text { (505-1372 SEC.) } \\
& 867.4 \\
& .976 / .982 \\
& 3.86(6.22) \\
& 559.7(15.85) \\
& .28(.01) \\
& 8096 .(229.3) \\
& 8.4 / 2 / \quad 8.40 \\
& 7.0121 \quad 7.00 \\
& 5.7 / 12 / 5.44 \\
& 75.8 / 14 / \quad 5871 \\
& 13.8 / 14 / .0470 \\
& \begin{array}{rrr}
13.8 / & 14 / & .0430
\end{array} \\
& .8 / 1 / \quad .21 \\
& 4.58 \\
& 22.47 \\
& 1.71 \\
& 4.82 \\
& 5422 \\
& 6.92 \\
& 1.46 \\
& .07 \\
& .236 \\
& \text { 1. } 286 \\
& \begin{array}{r}
1.286 \\
2275.95
\end{array} \\
& 3.128 \\
& .223 \\
& .010 \\
& 14.66 ; 16.05)
\end{aligned}
$$

RESEARCH

PROJECT NO. 08-6068-001

$$
\text { GASOLINE PHASE II EM-1701-F }
$$

FUEL DENSITY 6.186 LB/GAI

H .139 C .8410 .020 X .000

NOX HUMIDITY C.F. 1.030

3
HOT TRANSIENT
$0-505$ SEC
505.0

$.974 / .982$

$3.62(5.82)$

$559.8(15.86)$

$.27(.01)$

4714. (133.5)

$11.5 / 2 / 11.49$

$6.3 / 2 / \quad 6.30$

$65.5 / 13 / 154.52$

$.6 / 13 / 1.31$

$83.7 / \quad 14 / \quad .7291$

$14.0 \% 14 / 0478$

$55.7 /$ if 13.97

$1.1 /$ I/ .28

5.30

3.09

17.75

5.55

147.82

.6840

13.70

2.38

2.88

.439

22.975

1671.95

3.604

.212

.222

$18.30(12.85)$

$\begin{array}{ll}\text { CH4 } & \text { G/MI } \\ \text { NMHC } & \text { G/MI } \\ \text { CARBONIL } & \text { G/MI } \\ \text { ALCOHOL } & \text { G/MI }\end{array}$

.065
.178
.005
.000 
COMPUTER PROGRAM LDT $1.5-\mathrm{R}$

$\begin{array}{lcl}\text { VEHICLE NOMBER } & 53 \mathrm{M} \\ \text { VEHICLE MODEL } & 93 \text { CHEVY LUMINA } \\ \text { ENGINE } & 3.1 \mathrm{I}(189 \text { CID) }-\mathrm{V}-6 \\ \text { TRANSMISSION } & \text { L4 } \\ \text { ODOMETER } & 6787 \text { MILES ( } 10920 \mathrm{kM})\end{array}$

BAROMETER 29.23 IN HG $(742.4 \mathrm{MM} \mathrm{HG})$ RELATIVE HUMIDITY 51.9 PCT.

BAG NUMBER

BAG DESCRIPTION

RUN TIME SECONDS

DRY/WET CORRECTION FACTOR, SAMP/BACK

MEASURED DISTANCE MILES (KM)

BLOWER FLOW RATE SCFM (SCMM)

GAS METER FLOW RATE SCFM (SCMM)

TOTAL FLOW SCF (SCM)

HC SAMPLE METER/RANGE/PPM (BAG)

HC BCKGRD METER/RANGE/PPM

CO SAMPLE METER/RANGE/PPM

CO BCKGRD METER/RANGE/PPM

CO2 SAMPLE METER/RANGE/PCT

$\mathrm{CO} 2$ BCKGRD METER/RANGE/PCT

NOX SAMPLE METER/RANGE/PPM

NOX BCKGRD METER/RANGE/PPM

CH4 SAMPLE PPM $(1.120)$

CH4 BCKGRD PPM

\section{DILUTION FACTOR}

HC CONCENTRATION PPM

CO CONCENTRATION PPM

$\begin{array}{ll}\mathrm{CO} & \text { CONCENTRATION PPM } \\ \mathrm{CO} 2 & \text { CONCENTRATION PCT }\end{array}$

NOX CONCENTRATION PPM

CH4 CONCENTRATION PPM

NMHC CONCENTRATION PPM

$\begin{array}{ll}\text { THC } & \text { MASS GRAMS } \\ \text { CO } & \text { MASS GRAMS } \\ \text { CO2 } & \text { MASS GRAMS } \\ \text { NOX } & \text { MASS GRAMS } \\ \text { CH4 } & \text { MASS GRAMS } \\ \text { NMHC } & \text { MASS GRAMS (FID) } \\ \text { FUEL MASS KG } & \\ \text { FUEL ECONOMY MPG (L/10OKM) }\end{array}$

$\begin{array}{rrrr} & 89.3 / & 14 / & .8504 \\ 13.5 / & 14 / & .0458 \\ \text { (BAG) (D) } 96.7 / & 1 / & 24.08\end{array}$

$\begin{array}{lll}.71 & 1 / \quad .18\end{array}$

5.59

2.78

15.25

35.71

126.80

.8076

23.91

2.99

32.36

2.865

19.639

1967.22

5.983

.265

2. 482

651

$15.51(15.17\}$

3-BAG COMPOSITE RESULTS

$\begin{array}{llr}\text { THC } & \text { G/MI } & .245 \\ \text { CO } & \text { G/MI } & 3.773 \\ \text { NOX } & \text { G/MI } & .996\end{array}$

FUEL ECONOMY MPG (L/100KM)
RESEARCH

PROJECT NO. 08-6068-001

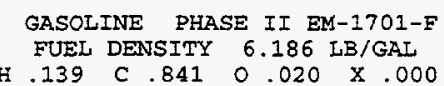

H $.139 \mathrm{C} .8410 .020 \mathrm{X} .000$

NOX HUMIDITY C.F. $\quad .984$

3

HOT TRANSIENT

( $0-505$ SEC.

505.4

$.977 / .984$

$3.61(5.81)$

$557.7(15.80)$

$.27(.01)$

4700. (133.1)

$$
.28(.01)
$$

$558.4(15.81)$

$8076 .(228.7)$

$81.9 / \quad 1 / \quad 8.22$

$67.3 / 1 / 6.75$

$4.6 / 12 / 4.38$

$1.1 / 12 / 1.04$

$75.7 / \quad 14 / .5855$

$13.3 / 14 / .0450$

$27.1 / 1 / 6.85$

$.9 / \quad 1 / \quad .23$

4.27

2.65

$\begin{array}{rrr}12.9 / & 2 / & 12.89 \\ 6.2 / & 2 / & 6.20 \\ 90.2 / & 13 / & 221.00 \\ .5 / & 13 / & 1.09 \\ 83.0 / & 14 / & .7153 \\ 13.5 / & 14 / & .0458 \\ 56.8 / & 1 / & 14.24 \\ .8 / & 1 / & .21 \\ & 5.06 & \\ & 2.58 & \end{array}$

22.54

1.76

3.28

.5425

6.62

1. 74

$-.19$

17.92

7.04

213.15

.6720

14.05

2.62

4.10

$$
\begin{array}{r}
.280 \\
.873 \\
2271.64 \\
2.850 \\
.266 \\
.000
\end{array}
$$

$14.64(16.07)$

$$
\begin{array}{r}
.555 \\
33.032 \\
1637.84 \\
3.518 \\
.233 \\
.315 \\
.549
\end{array}
$$

$18.46(12.74)$

$\begin{array}{lll}\text { CH4 } & \text { G/MI } & .069 \\ \text { NMIC } & \text { G/MI } & .167 \\ \text { CARBONYL } & \text { G/MI } & .006 \\ \text { ALCOHOL } & \text { G/MI } & .003\end{array}$


COMPUTER PROGRAM LDT $1.5-\mathrm{R}$

SOUTHWEST RESEARCH INSTITUTE - DEPARTMENT OE EMISSIONS RESEARCH 3-BAG CARB FTP VEHICLE EMISSION RESULTS

VEAICLE NUMBER 53

VEHICLE MODEL 93 CHEVY LUMINA

ENGINE

6630 MILES ( $10667 \mathrm{KM}$ )

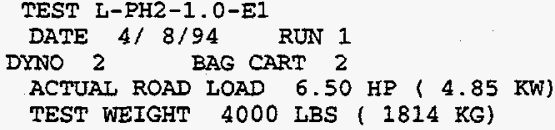

3.1 I $(189$ CID $)-V-6$

BAROMETER 29.27 IN HG $(743.5 \mathrm{MM} \mathrm{HG})$

RELATIVE HUMIDITY 45.7 PCT.

BAG NUMBER

BAG DESCRIPTION

RUN TIME SECONDS

DRY/WET CORRECTION FACTOR, SAMP/BACK

MEASURED DISTANCE MILES (KM)

BLOWER FLOW RATE SCFM (SCMM)

GAS METER FLOW RATE SCFM (SCMM)

TOTAL FLOW SCF (SCM)

HC SAMPLE METER/RANGE/PPM (BAG)
HC BCKGRD METER/RANGE/PPM
CO SAMPLE METER/RANGE/PPM
CO BCKGRD METER/RANGE/PPM
CO2 SAMPLE METER/RANGE/PCT
CO2 BCKGRD METER/RANGE/PCT
NOX SAMPLE METER/RANGE/PPM (BAG) (D)
NOX BCKGRD METER/RANGE/PPM
CH4 SAMPLE PRM (1.100)
CH4 BCKGRD PPM
DILUTION FACTOR
HC CONCENTRATION PPM
CO CONCENTRATION PPM
CO2 CONCENTRATION PCT.
NOX CONCENTRATION PPM
CH4 CONCENTRATION PPM
NMHC CONCENTRATION PPM
THC MASS GRAMS
CO MOS GRAMS
CO2 M MASS GRAMS
NOX MASS GRAMS
CH4 MASS GRAMS
NMHC MASS GRAMS (FID)
FUEI MASS KG
FUEI ECONOMY MPG (L/10OKM)

DRY BULB TEMPERATURE $77.0^{\circ} \mathrm{F}\left(25.0^{\circ} \mathrm{C}\right)$

$$
\begin{gathered}
1 \\
\text { COID TRANSIENT } \\
(0-505 \text { SEC. }) \\
506.0 \\
.9781 .985 \\
3.62(5.83) \\
557.9(15.80) \\
.28(1.01) \\
4707 .(133.3)
\end{gathered}
$$$$
\begin{array}{rrr}
12.4 / & 3 / & 123.72 \\
.7 / & 3 / & 6.98 \\
53.3 / & 1 / & 486.56 \\
.0 / & 1 / & .00 \\
81.9 / & 14 / & .6941 \\
12.9 / & 14 / & .0434 \\
43.2 / & 2 / & 43.22 \\
.3 / & 2 / & .30 \\
& 7.10 & \\
& 3.13 &
\end{array}
$$

17.54

117.14

472.67

.6531

42.94

4.15

112.57

9.727

73.359

1594.11

10.445

.369

8.653

.565

$18.01 ;(13.06)$

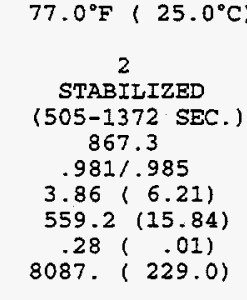

$81.5 / 2 / \quad 81.45$

$\begin{array}{rrr}6.8 / 21 & 21 & 6.80\end{array}$

$54.2 / 14 / 243.66$

$.5 / 14 / 2.02$

$68.6 / 14 / .4801$

$13.4 / 14 / .0454$

$75.5 / 1 / 18.89$

$\begin{array}{lll}.8 / & 11 & .21\end{array}$

5.37

3.20

25.83

74.92

235.83

.4365

18.69

2.30
72.40

10.754
62.877

62.877
1830.04

7.812

.350

9.560

.637

$16.99 ;(13.84)$

$\mathrm{CH} 4$

NMHC

G/MI

CARBONYL G/MI

ALCOHOL G/MI
PROJECT NO. 08-6068-001

GASOLINE PHASE II EM 1701-F FUEI DENSITY $6.186 \mathrm{LB} / \mathrm{GAI}$ $\begin{array}{lllll}\text { H. } & .139 & C .841 & 0.020 & \text { X } .000\end{array}$

NOX HUMIDITY C.F. .954

HOT TRANSIENT

( $0-505$ SEC.)

504.9

$.979 / .985$

$3.62(5.83)$

$562.6(15.93)$

$.28(.01)$

4736. ( 134.1$)$

$9.7 / 3 / \quad 96.78$

$.8 / 3 / 7.98$

$82.0 / 14 / 398.47$

$.7 / 24 / 2.83$

$78.3 / 14 / \quad .6289$

$14.0 / 14 / \quad .0478$

$\begin{array}{lll}36.7 / 2 / 2 & 36.72\end{array}$

.3/ 21

5.90

3.25

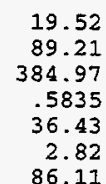

7. 471

60.117

1433.06

8. 918

.252

6.660

503

$20.19 ;(11.65)$

$\begin{array}{llr}\text { THC } & \text { G/MI } & 2.566 \\ \text { CO } & \text { G/MI } & 17.205 \\ \text { NOX } & \text { G/MI } & 2.325\end{array}$

FUEL ECONOMY MPG (L/100KM)
$18.00(13.07)$ 
COMPUTER PROGRAM IDT $1.5-R$

VEHICLE NUMBER $53 \mathrm{M}$

VEHICLE MODEL 93 CHEVY LUMINA

ENGINE

TRANSMISSION

ODOMETER

3.1 L (189 CID) $-V-6$

L4

6649 MILES ( $10698 \mathrm{KM}$ )

BAROMETER 29.03 IN HG $(737.4 \mathrm{MM} \mathrm{HG})$ RELATIVE HUMIDITY 65.9 PCT.

BAG NUMBER

BAG DESCRIPTION

RUN TIME SECONDS

DRY/WET CORRECTION FACTOR, SAMP/BACK

MEASURED DISTANCE MILES (KM)

BLOWER FLOW RATE SCFM (SCMM)

GAS METER FLOW RATE SCFM (SCMM)

TOTAL FLOW SCF (SCM)

HC SAMPLE METER/RANGE/PPM (BAG)

HC BCKGRD METER/RANGE/PPM

CO SAMPIE METER/RANGE/PPM

CO BCKGRD METER/RANGE/PPM

CO2 SAMPLE METER/RANGE/PCT

CO2 BCKGRD METER/RANGE/PCT

NOX SAMPLE METER/RANGE/PPM

NOX BCKGRD METER/RANGE/PPM

CH4 SAMPLE PPM $(1.100)$

CH4 BCKGRD PPM

(BAG) (D)

DILUTION FACTOR

HC CONCENTRATION PPM

CO CONCENTRATION PPM

$\mathrm{CO} 2$ CONCENTRATION PCT

NOX CONCENTRATION PPN

CH4 CONCENTRATION PPM

INMHC CONCENTRATION PPM

$\begin{array}{lll}\text { THC } & \text { MASS GRAMS } \\ \text { CO } & \text { MASS GRAMS } \\ \text { CO2 } & \text { MASS GRAMS } \\ \text { NOX } & \text { MASS GRAMS } & \\ \text { CH4 } & \text { MASS GRAMS } & \\ \text { NMHC } & \text { MASS GRAMS } & \text { (FID) } \\ \text { FUEI } & \text { MASS KG } & \end{array}$

FUEI, MASS KG

FUEL ECONOMY MPG (L/100KM)

\begin{abstract}
3-BAG COMPOSITE RESULTS
\end{abstract}

$\begin{array}{llr}\text { THC } & \text { G/MI } & 2.701 \\ \text { CO } & \text { G/MI } & 17.390 \\ \text { NOX } & \text { G/MI } & 2.259\end{array}$

FUEL ECONOMY MPG (L/10OKM) 17.93 (13.12)
3-BAG CARB FTP VEHICLE EMISSION RESULTS

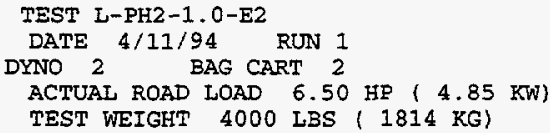

DRY BULB TEMPERATURE $74.0^{\circ} \mathrm{F}\left(23.3^{\circ} \mathrm{C}\right)$

$$
1
$$

COLD TRANSIENT

( 0 -505 SEC.

506.0

$.974 / .981$

$3.62(5.82)$

$552.6(15.65)$

$27(.01)$

4662 . ( 132.0$)$

$13.7 / 3 / 136.70$

$.8 / 3 / 7.98$

$56.1 / 1 / 516.59$

$.2 / 1 / 1.68$

$82.7 / \quad 14 / \quad .7094$

$13.1 / 14 / .0442$

$39.9 / 2 / \quad 39.92$

$\begin{array}{lll}.31 & 21 & .30\end{array}$

7.49

3.23

17.11

129.18

496.78

.6678

39.64

4.44

124.29

10.520

76.361

1614.30

10.533

.391

9.463
.573

$17.70(13.29)$

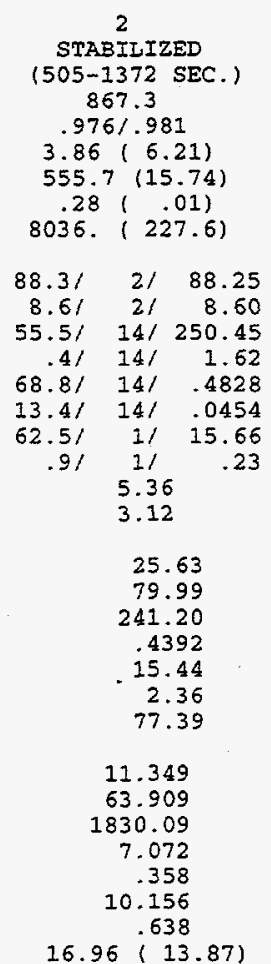

$\mathrm{CH} 4$

NMIC

G/MI

$G / M I$

ARBONYL $G / M$

ALCOHOL G/MI
RESEARCH

PROJECT NO. 08-6068-001

GASOLINE PHASE II EM-1701-F FUEL DENSITY $6.186 \mathrm{IB} / \mathrm{GAL}$ H .139 C .8410 .020 X .000

NOX HUMIDITY C.F. 1.052

HOT TRANSIENT

( $0-505$ SEC.

505.8

$974 / .981$

$3.62(5.82)$

$555.6 \quad(15.74)$

$27(.01)$

4686. ( 132.7$)$

$10.1 / 3 / 100.78$

$1.013 / \quad 9.98$

$81.0 / 14 / 392.64$

$.5 / 14 / 2.02$

$78.6 / 14 / .6341$

$13.9 / 14 / .0474$

$34.9 / 2 / 34.92$

$\begin{array}{lll}21 & 21 & .20\end{array}$

5.93

2.99

19.39

91.31

377.47

.5891

34.73

3.10

87.91

7.571

58.322

1431.51

9.276

.274

6.727

.502

$20.22 ;(11.63)$ 
COMPUTER PROGRAM LDT $1.5-\mathrm{R}$

VBHICLE NUMBER $53 \mathrm{M}$

VEHICLE MODEL 93 CHEVY LUMINA

ENGINE

3.1 L (189 CID) -V-

14

6601 MILES ( $10621 \mathrm{KM}$ )

ODOMETER

BAROMETER 29.32 IN HG (744.7 MM HG)

RELATIVE HUMIDITY $26.4 \mathrm{PCT}$.

BAG NUMBER

BAG DESCRIPTION

RUN TIME SECONDS

DRY/WET CORRECTION FACTOR, SAMP/BACK

MEASURED DISTANCE MILES (KM)

BLOWER FLOW RATE SCFM (SCMM)

GAS METER FLOW RATE SCFM (SCMM)

TOTAL FLOW SCF (SCM)

HC SAMPLE METER/RANGE/EPM (BAG)

HC BCKGRD METER/RANGE/PPM

CO SAMPLE METER/RANGE/PPM

CO BCKGRD METER/RANGE/PPM

CO2 SAMPLE METER/RANGE/PCT

CO2 BCKGRD METER/RANGE/PCT

NOX SAMPLE METER/RANGE/PPM (BAG) (D)

NOX BCKGRD METER/RANGE/PPM

CH4 SAMPLE PPM $(1.100)$

CH4 BCKGRD PPM

DIIUTION FACTOR

HC CONCENTRATION PPM

CO CONCENTRATION PPM

$\mathrm{CO} 2$ CONCENTRATION PCT

NOX CONCENTRATION PPM

CH4 CONCENTRATION PPM

NMHC CONCENTRATION PPM

THC MASS GRAMS

CO MASS GRAMS

$\mathrm{CO} 2$ MASS GRAMS

NOX MASS GRAMS

CH4 MASS GRAMS

MMILC MASS GRAMS (FID)

FUEL MASS KG

FUEI ECONOMY MPG (L/100KM)

-BAG COMPOSITE RESULTS

$\begin{array}{llr}\text { THC } & \text { G/MI } & .242 \\ \text { CO } & \text { G/MI } & 4.100 \\ \text { NOX } & \text { G/MI } & .360\end{array}$

FUEL ECONOMY MPG (L/100KM) 18.24 (12.90)
3-BAG CARB FTP VEHICLE EMISSION RESULTS

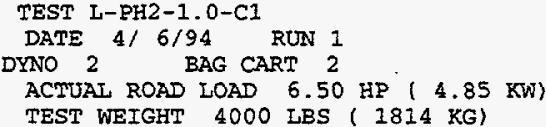

DRY BULB TEMPERATURE $70.0^{\circ} \mathrm{F}\left(21.1^{\circ} \mathrm{C}\right)$

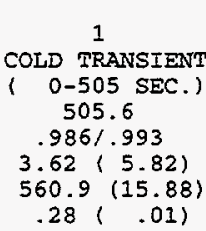

4729. ( 133.9$)$

$44.41 \quad 2 / \quad 44.37$

$\begin{array}{lll}6.31 & 21 & 6.30\end{array}$

$62.5 / 14 / 287.87$

$.2 / 14 / \quad .81$

$83.6 / 14 / \quad .7271$

$12.6 / 14 / \quad .0422$

$57.5 / \quad 1 / \quad 14.42$

$.61 .11 \quad .15$

5.41

2.53

17.41
38.44

280.51

.6873

14.27

3.03
35.10

3.103

43.734

1685.18

3.009

.271

2.711

$17.73(13.27)$

STABILIZED

(505-1372 SEC. 867.6

$988 / .993$

$3.90(6.27)$

$563.3(15.95)$

$28\{.01\}$

$8149 .(230.8)$

$\begin{array}{lll}70.6 / & 1 / & 7.08\end{array}$

$63.7 / \quad 1 / 6.39$

$9.4 / 12 / 9.00$

$.3 / 12 / \quad 28$

$70.1 / 14 / .5009$

$12.9 / 14 / \quad .0434$

$10.8 /$ 1/ 2.76

$.6 / 1 / 2.15$

2.97

26.32

.94

8.56

4591

2. 61

.51

.130

2.300

1939.87

.948

.079

.050

.631

$17.32 ; 13.58\}$

$\mathrm{CH} 4$

NMHC

G/MI

$G / M I$

CARBONYI G/MI

ALCOHOL G/MI
RESEARCH

PROJECT NO. 08-6068-001

GASOLINE PHASE II EM-1701-F

FUEI DENSITY 6.186 LB/GAI

H .139 C .841 $0.020 \quad$ X .000

NOX HUMIDITY C.F. $\quad .823$

HOT TRANSIENT

( $0-505$ SEC.)

505.7

$.987 / .993$

$3.64(5.85)$

$562.7(15.93)$

$.28(.01)$

4745. (134.4)

$13.6 / 2 / 13.59$

6.0/. $2 / 6.00$

$48.6 / 13 / 111.89$

$.2 / \quad 13 / \quad .44$

$79.4 / \quad 14 / \quad .6481$

$12.7 / 14 / .0426$

$15.4 / 3 / 3.92$

.2) $1 /$

4.05

2.55

20.02

7.90

109.08

.6076

3.87

1.62

6.11

.621

17.064

1494.85

.818

.145

.473

$20.63 \div 11.40)$ 
COMPUTER PROGRAM LDT $1.5-R$

VEHICLE NUMBER 53M

VEHICLE MODEL 93 CHEVY LUMINA

ENGINE $\quad 3.1$ I (189 CID) $-V-5$

TRANSMISSION IA

ODOMETER 6612 MILES ( $10638 \mathrm{kM})$

BAROMETER 29.44 IN HG $(747.8 \mathrm{MM} \mathrm{HG)}$ REIATIVE HUMIDITY $27.0 \mathrm{PCT}$.

BAG NUMBER

BAG DESCRIPTION

RUN TIME SECONDS

DRY/WET CORRECTION FACTOR, SAMP/BACK

MEASURED DISTANCE MILES (KM)

BLOWAR FLOW RATE SCFM (SCMM)

GAS METER FLOW RATE SCFM (SCMM)

TOTAL FLOW SCF (SCM)

HC SAMPLE METER/RANGE/PPM (BAG)

HC BCKGRD METER/RANGE/PPM

CO SAMPLE METER/RANGE/PPM

CO BCKGRD METER/RANGE/PRM

$\mathrm{CO} 2$ SAMPLE METER/RANGE/PCT

CO2 BCKGRD METER/RANGE/PCT

NOX SAMPLE METER/RANGE/PPM (BAG) (D)

NOX BCKGRD METER/RANGE/PPM

CH4 SAMPLE PPM $(1.100)$

CH4 BCKGRD PRM

DILUTION FACTOR

HC CONCENTRATION PPM

CO CONCENTRATION PPM

$\mathrm{CO} 2$ CONCENTRATION PCT

NOX CONCENTRATION PPM

CH4 CONCENTRATION PPM

NMIC CONCENTRATION PPM

$\begin{array}{lll}\text { THC } & \text { MASS GRAMS } \\ \text { CO } & \text { MASS GRAMS } \\ \text { CO2 } & \text { MASS GRAMS } \\ \text { NOX } & \text { MASS GRAMS } \\ \text { CH4 } & \text { MASS GRAMS } \\ \text { NMHC } & \text { MASS GRAMS (FID) } \\ \text { FUEL } & \text { MASS KG }\end{array}$

FUEL MASS KG

FUEL ECONOMY MPG (L/100KM)

3-BAG COMPOSITE RESULTS

$\begin{array}{llr}\text { THC } & \text { G/MI } & .253 \\ \text { CO } & \text { G/MI } & 4.308 \\ \text { NOX } & \text { G/MI } & .311\end{array}$

FUEL ECONOMY MPG (L/100KM)
3-BAG CARB FTP VEHICLE EMISSION RESULTS

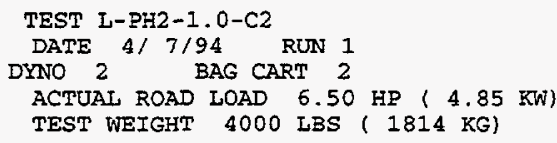

DRY BULB TEMPERATURE $74.0^{\circ} \mathrm{F}\left(23.3^{\circ} \mathrm{C}\right)$
1

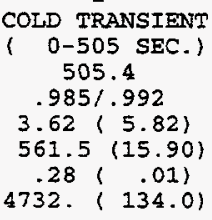

$42.6 / 2 / \quad 42.58$

$5.6 / 2 / 5.60$

$91.3 / 13 / 224.08$

$.8 / 13 / 21.74$

$84.1 / 14 / .7371$

$13.4 / \quad 14 / \quad .0454$

$66.3 / 1 / 16.61$

$1.5 / 1 / \quad .39$

5.18

2.76

17.33
37.30

217.22

.6944

16.24

2.58

34.47

3.037

33.892

1703.72

3.493

.230
2.653

.573

$17.70\langle 13.29\}$

53

311

$18.05(13.03)$
STABILIZED

(505-1372 SEC.)

867.3

$.987 / .992$

$3.87(6.22)$

$562.9(15.94)$

$28(.01)$

8142 . (230.6)

$63.9 / 61 / 6.41$

$54.2 / \quad 1 / 5.44$

$14.8 / 12 / 14.23$

$1.9 / 12 / 1.81$

$70.3 / 14 / \quad .5037$

$13.5 / 14 / \quad .0458$

$6.1 / 1 / 1.56$

$1.3 / 1 / \quad .34$

3.29

2.74

26.15

1.18

12.24

.4597

1.24

.65

.162
3.286

1940.48

.459

.100

.062

.632

$17.18(13.69)$

RESEARCH

RROJECT NO. 08-6068-001

GASOLINE PHASE II EM-1701-F

FUEL DENSITY $6.186 \mathrm{LB} / \mathrm{GAL}$

$\begin{array}{lllllll}\text { H } & .139 & \mathrm{C} & .841 & 0 & .020 & \mathrm{X} \\ .000\end{array}$

NOX HUMIDITY C.F. $\quad .839$

$$
3
$$

HOT TRANSIENT

( 0- 505 SEC.)

505.6

$.986 / .992$

$3.64(5.85)$

$564.1(15.98)$

$27(.01)$

4756. (134.7)

$14.9 / 2 / \quad 14.89$

$5.6 / 2 / 5.60$

$70.2 / 13 / 166.78$

$.8 / 13 / 1.74$

$80.0 / 14 / .6589$

$13.9 / 14 / \quad .0474$

$12.4 / \quad 1 / 3.16$

.81 1/ .21

4.38

2.74

$$
\begin{array}{r}
19.54 \\
9.58 \\
161.51 \\
.6139 \\
2.97 \\
1.78 \\
7.62 \\
.754 \\
25.324 \\
1513.85 \\
.641 \\
.160 \\
.592
\end{array}
$$

$20.21(11.64)$ 
SOUTHWEST RESEARCH INSTITUTE - DEPARTMENT OF EMISSIONS RESEARCH 3-BAG CARB FTP VEHICLE EMISSION RESULTS

VEHICLE NUMBER $53 \mathrm{M}$

VEHICLE MODEL 93 CHEVY LUMINA

ENGINE

TRANSMISSION

I4

6707 MILES ( $10791 \mathrm{kM})$

BAROMETER 29.08 IN HG $(738.6 \mathrm{MM} \mathrm{HG})$

RELATIVE HUMIDITY 62.4 PCT.

BAG NTMBER

BAG DESCRIPTION

RUN TIME SECONDS

DRY/WET CORRECTION FACTOR, SAMP/BACK

MEASURED DISTANCE MIIES (KM)

BLOWER FLOW RATE SCFM (SCMM)

GAS METER FIOW RATE SCFM (SCMM)

TOTAL FLOW SCF (SCM)

\begin{tabular}{|c|c|c|}
\hline $\mathrm{HC}$ & SAMPLE METER/RANGE/PPM & (BAG) \\
\hline HC & BCKGRD METER/RANGE/PPM & \\
\hline $\mathrm{CO}$ & SAMPLE METER/RANGE/PPM & \\
\hline $\mathrm{CO}$ & BCKGRD METER/RANGE/PPM & \\
\hline $\mathrm{CO} 2 s$ & SAMPLE METER/RANGE/PCT & \\
\hline $\mathrm{CO} 2 \mathrm{E}$ & BCKGRD METER/RANGE/PCT & \\
\hline ox $s$ & SAMPLE METER/RANGE/PPM & (BAG) (D) \\
\hline $\mathrm{OX}$ & BCKGRD METER/RANGE/PPM & \\
\hline H4 s & SAMPLE PPM $(1.120)$ & \\
\hline & BCKGRD PPM & \\
\hline \multicolumn{3}{|c|}{ DILUTION FACTOR } \\
\hline $\mathrm{C}$ & CONCENTRATION PPM & \\
\hline 0 & CONCENTRATION PPM & \\
\hline 02 & CONCENTRATION PCT & \\
\hline ox & CONCENTRATION PPM & \\
\hline H4 & CONCENTRATION PPM & \\
\hline MHC & C CONCENTRATION PPM & \\
\hline HC & MASS GRAMS & \\
\hline & MASS GRAMS & \\
\hline 02 & MASS GRAMS & \\
\hline ox & MASS GRAMS & \\
\hline 14 & MASS GRAMS & \\
\hline MHC & MASS GRAMS (FID) & \\
\hline $\begin{array}{l}\text { FUEL } \\
\text { FUEL }\end{array}$ & $\begin{array}{l}\text { MASS KG } \\
\text { ECONOMY MPG }(L / 100 \mathrm{KM})\end{array}$ & \\
\hline
\end{tabular}

DYNO 2 BAG CART

$$
\begin{gathered}
1 \\
\text { COLD TRANSIENT } \\
(0-505 \text { SEC. }) \\
506.0 \\
.975 / .981 \\
3.62(5.82) \\
553.1(15.66) \\
.27\left(\begin{array}{c}
.01) \\
4667 .(132.2)
\end{array}\right.
\end{gathered}
$$

$17.1 / 3 / 170.62$

.9/ 318.98

$83.4 / 2 / 2243.07$

$.3 / 2 / 4.70$

$79.5 / 14 / .6499$

$15.8 / 14 / .0552$

$89.5 / 1 / 22.33$

14.33

.43

14.96

162.24

2164.60

.5984

22.07

11.13

149.77

12.868

333.068

1448.01

5.817

.981

11.414

653

$15.56 \div(15.12)$
PROJECT NO. 08-6068-001

GASOLINE PHASE II EM-1701-F

FUEI DENSTTY 6.186 LB/GAT

H .139 C $8410.020 \times .000$

ACTUAL ROAD IOAD $6.50 \mathrm{HP}(4.85 \mathrm{~kW}$

TEST WEIGHT 4000 LBS ( $1814 \mathrm{KG}$ )

DRY BULB TEMPERATURE $75.0^{\circ} \mathrm{F}\left(23.9^{\circ} \mathrm{C}\right)$

$$
\begin{aligned}
& 2 \\
& \text { STABIIIZED } \\
& \text { (505-1372 SEC. } \\
& 867.2 \\
& .977 / .981 \\
& 3.87(6.22) \\
& 556.0(15.75) \\
& .27(.01) \\
& 8039 \text {. (227.7) }
\end{aligned}
$$

CH4 G/MI

NMHC $G / M I$

CARBONYL G/MI

ALCOHOL G/MI
NOX HUMIDITY C.F. 1.043

$$
\begin{aligned}
& 3 \\
& 505.6 \\
& .976 / .981
\end{aligned}
$$

16.32

149.22

2656.45

.4773

9.20

12.78

134.91

$$
\begin{array}{r}
11.759 \\
409.753 \\
1157.74 \\
2.431 \\
1.129 \\
10.307 \\
.597
\end{array}
$$

$17.01 ; 13.83\}$
.377

3.443

.089

3-BAG COMPOSITE RESULTS

$\begin{array}{llr}\text { THC } & \text { G/MI } & 3.938 \\ \text { CO } & \text { G/MI } & 119.916 \\ \text { NOX } & \text { G/MI } & .849\end{array}$

FUEL ECONOMY MPG (I/100KM) 
COMPUTER PROGRAM LDT 1.5-R

$\begin{array}{lc}\text { VEHICLE NUMBER } & 53 \mathrm{M} \\ \text { VEHICLE MODEL } & 93 \text { CHEVY LUMINA } \\ \text { ENGINE } & 3.1 \mathrm{~L} \text { (189 CID) -V-6 } \\ \text { TRANSMISSION } & \text { L4 } \\ \text { ODOMETER } & 6718 \text { MILES ( } 10809 \mathrm{KM})\end{array}$

BAROMETER 29.04 IN HG $(737.6 \mathrm{mM} \mathrm{HG})$ REIATIVE HUMIDITY 62.5 PCT.

BAG NUMBER

BAG DESCRIPTION

RUN TIME SECONDS

DRY/WET CORRECTION FACTOR, SAMP/BACK

MEASURED DISTANCE MILES (KM)

BLOWER FLOW RATE SCFM (SCMM)

GAS METER FLOW RATE SCFM (SCMM)

TOTAL FLOW SCF (SCM)

HC SAMPLE METER/RANGE/PPM (BAG)

HC BCKGRD METER/RANGE/PPM

CO SAMPLE METER/RANGE/PRM

CO BCKGRD METER/RANGE/PPM

$\mathrm{CO} 2$ SAMPLE METER/RANGE/PCT

$\mathrm{CO} 2$ BCKGRD METER/RANGE/PCT

NOX SAMPLE METER/RANGE/PPM (BAG) (D)

NOX BCKGRD METER/RANGE/PPM

CH4 SAMPLE PPM $(1.120)$

CHA BCKGRD PPM

\section{DILUTION FACTOR}

HC CONCENTRATION PPM

CO CONCENTRATION PPM

$\mathrm{CO} 2$ CONCENTRATION PCT

NOX CONCENTRATION PPM

CH4 CONCENTRATION PPM

NMHC CONCENTRATION PPM

$\begin{array}{ll}\text { THC } & \text { MASS GRAMS } \\ \text { CO } & \text { MASS GRAMS } \\ \text { CO2 } & \text { MASS GRAMS } \\ \text { NOX } & \text { MASS GRAMS } \\ \text { CH4 } & \text { MASS GRAMS } \\ \text { NMHC } \\ \text { MASS GRAMS (FID) } \\ \text { FUEL MASS KG } \\ \text { FUEL ECONOMY MPG (L/100KM) }\end{array}$

SOUTHWEST RESEARCH INSTITUTE - DEPARTMENT OF EMISSIONS RESEARCH

3-BAG CARB FTP VEAICLE EMISSION RESULTS

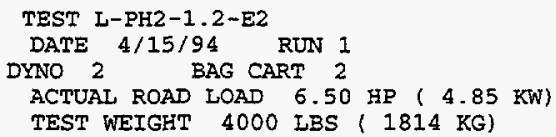

DRY BULB TEMPERATURE $75.0^{\circ} \mathrm{F}\left(23.9^{\circ} \mathrm{C}\right)$

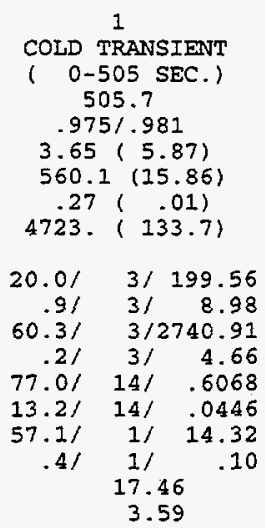

14.83

191.18

2648.35

.5652

14.22

14.11

175.38

15.202

412.367

1384.07

3.796

1. 258

13.525

.675

$15.17(15.51)$

$$
.28(.01)
$$

$560.3(15.87)$

8101 . (229.4)

$\begin{array}{rrr}15.1 / & 3 / & 150.65 \\ 1.0 / & 3 / & 9.98 \\ 80.9 / & 2 / 2135.88 \\ .5 / & 2 / & 7.83 \\ 61.8 / & 14 / & .3943 \\ 13.5 / & 14 / & .0458 \\ 21.2 / & 1 / & 5.37 \\ .7 / & 1 / & .18 \\ & 16.40 & \\ & 3.64 & \end{array}$

21.44

141.15

2068.76

.3506

5.20

12.93

126.57

19.202

552.536

1472.74

2.381

1.977

16.757

.779

$14.04(16.75)$
PROJECT NO. 08-6068-001

GASOLINE PHASE II EM-1701-F FUEL DENSITY 6.186 LB/GAL. H .139 C .841 0.020 X .000

NOX HUMIDITY C.F. 1.044

HOT TRANSIENT

$(0-505$ SEC. $)$

506.4

$.976 / .981$

$3.65(5.88)$

559.3 (15.84)

$.27(.01)$

4723 . ( 133.8 )

$16.8 / 3 / 167.63$

$1.1 / 3 / \quad 10.98$

$61.6 / 3 / 2841.46$

$.4 / 3 / 9.34$

$\begin{array}{rrr}71.3 / & 14 / & .5181\end{array}$

$13.3 / 14 / .0450$

$38.2 / \quad 1 / 9.61$

$1.3 / \quad 1 / \quad .34$

17.16
2.95

16.32

157.32

2746.33

.4758

9.30

14.39

141.20

12.515

427.664

1165.28

2.482

1.283

10.891

.609

$16.83 ; 13.97)$

3-BAG COMPOSITE RESULTS

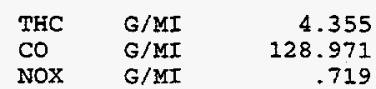

FUEL ECONOMY MPG (L/100KM)

$14.96(15.72)$

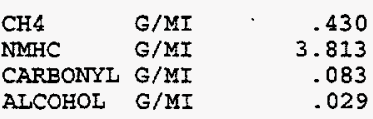


COMPUTER PROGRAM LDT 1.5-R

VEHICLE NUMBER 53M

VEHICLE MODEL 93 CHEVY IUMTNA

ENGTNE

3.1 L (189 CID) $-V-6$

3.1
$\mathbf{4} 4$

6736 MILES ( $10838 \mathrm{kM})$

ODOMETER

REIATIVE HUMIDITY 42.3 PCT.

BAG NUMBER

BAG DESCRIPTION

RUN TIME SECONDS

DRY/WET CORRECTION FACTOR, SAMP/BACK

MEASURED DISTANCE MILES (IM)

BLOWER FLOW RATE SCFM (SCMM)

GAS METER FLOW RATE SCFM (SCMM)

TOTAE FLOW SCF (SCM)

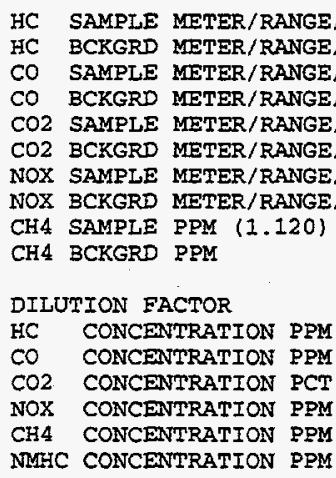

$\begin{array}{ll}\text { THC } & \text { MASS GRAMS } \\ \text { CO } & \text { MASS GRAMS } \\ \text { CO2 } & \text { MASS GRAMS } \\ \text { NOX } & \text { MASS GRAMS } \\ \text { CH4 } & \text { MASS GRAMS } \\ \text { NMHC } & \text { MASS GRAMS (FID) } \\ \text { FUEL } & \text { MASS KG }\end{array}$

FUEL ECONOMY MPG (L/10OKM)

(BAG)

3-BAG CARB FTR VEHICLE EMISSION RESULTS

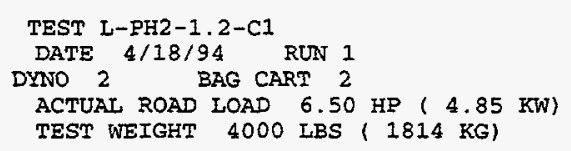

DRY BULB TEMPERATURE $77.0^{\circ} \mathrm{F}\left(25.0^{\circ} \mathrm{C}\right)$

$$
\begin{gathered}
1 \\
\text { COLD TRANSIENT } \\
(0-505 \text { SEC. }) \\
505.5 \\
.980 / .986 \\
3.53(5.84) \\
560.2(15.86) \\
.27\left(\begin{array}{c}
.01) \\
4722 .(133.7)
\end{array}\right.
\end{gathered}
$$$$
\begin{array}{rcr}
18.0 / & 3 / & 179.60 \\
.7 / & 3 / & 6.98 \\
91.1 / & 2 / 2589.53 \\
.5 / & 2 / & 7.83 \\
76.9 / & 14 / & .6051 \\
13.7 / & 14 / & .0466 \\
51.3 / & 1 / & 12.87 \\
.7 / & 1 / & .18 \\
& 16.21 & \\
& 3.48 &
\end{array}
$$

15.11

173.08

2515.80

.5616

12.70

12.96

158.56

\subsection{9}

391.639

1374.99

3.032

1.155

12.226

$15.42(15.25)$

$$
\begin{aligned}
& \stackrel{2}{\text { STABILIZED }} \\
& \text { (505-1372 SEC.) } \\
& \begin{array}{l}
866.8 \\
982 / .986
\end{array} \\
& 3.89(6.25) \\
& 562.3(15.92) \\
& .28(.01) \\
& 8127 \text {. ( 230.2) } \\
& 13.8 / 3 / 137.69 \\
& \begin{array}{rrr}
.8 / & 3 / 7.98
\end{array} \\
& 79.3 / 2 / 2068.74 \\
& \begin{array}{rrr}
.6 / & 27 & 9.40 \\
1.8 / & 14 / & .3943
\end{array} \\
& \begin{array}{lll}
61.8 / & 14 / & .3943 \\
14.0 / & 14 / & .0478
\end{array} \\
& \begin{array}{rrr}
14.0 / & 14 / & .0478 \\
8.1 / & 1 / & 2.07
\end{array} \\
& 1.01 .1 / 26 \\
& 15.57 \\
& 3.34 \\
& 21.66 \\
& 130.08 \\
& 2015.41 \\
& .3487 \\
& 1.83 \\
& 12.38 \\
& 116.21
\end{aligned}
$$

RESEARCH

PROJECT NO. 08-6068-001

GASOLINE PHASE II EM-1701-F

FUEL DENSITY $6.186 \mathrm{LB} / \mathrm{GAT}$

H .139 C . $8410.020 \times .000$

NOX HUMIDITY C.F. .933

$$
3
$$

HOT TRANSIENT

( $0-505$ SEC.

505.8

$981 / .986$

$3.63(5.85)$

$560.9(15.89)$

$.27(.01)$

4731. (134.0)

$\begin{array}{rrr}15.0 / & 3 / & 149.67 \\ .8 / & 3 / & 7.98 \\ 95.0 / & 2 / 2773.18 \\ .9 / & 2 / & 14.12 \\ 71.1 / & 14 / & .5152 \\ 14.3 / & 14 / & .0490 \\ 21.9 / & 1 / & 5.55 \\ 1.4 / & 1 / & .36 \\ 16.05 & \\ 3.20 & \end{array}$

16.51
142.17
2693.81
.4691
5.21
13.04
127.57

11.458

420.193
1150.80

1.246

1.164

9.856

.599

$17.01(13.83)$

3-EAG COMPOSITE RESULTS

$\begin{array}{llr}\text { THC } & \text { G/MI } & 4.035 \\ \text { CO } & \text { G/MI } & 126.102 \\ \text { NOX } & \text { G/MI } & .368\end{array}$

FUEL ECONOMY MPG (L/100KM)

$\begin{array}{llr}\text { CH4 } & \text { G/MI } & .407 \\ \text { NMHC } & \text { G/MI } & 3.498 \\ \text { CARBONYI G/MI } & .058 \\ \text { ALCOHOI } & \text { G/MI } & .071\end{array}$


SOUTHWEST RESEARCH INSTITUTE - DEPARTMENT OF EMISSIONS RESEARCH

3-BAG CARS FTP VEHICLE EMISSION RESULTS

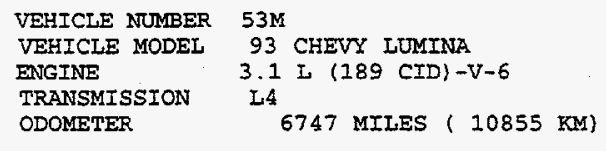

BAROMETER 29.28 IN HG (743.7 MM HG) RELATIVE HUMIDITY 60.9 PCT.

BAG NUMBER

BAG DESCRIPTION

RUN TIME SECONDS

DRY/WET CORRECTION EACTOR, SAMP/BACK

MEASURED DISTANCE MILES (KM)

BLOWER FLOW RATE SCFM (SCMM)

GAS METER FLOW RATE SCFM (SCMM)

TOTAL FLOW SCF (SCM)

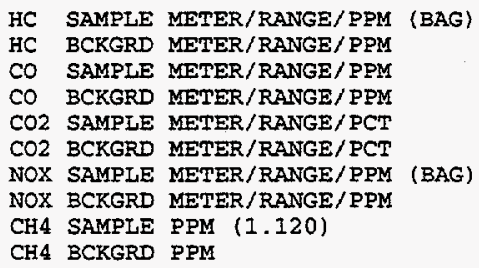

CH4 BCKGRD PPM

\section{DILUTION FACTOR}

HC CONCENTRATION PPM

CO CONCENTRATION PPM

$\mathrm{CO} 2$ CONCENTRATION PCT

NOX CONCENTRATION PPM

CH4 CONCENTRATION PPM

NMHC CONCENTRATION PPM

$\begin{array}{lll}\text { THC } & \text { MASS GRAMS } \\ \text { CO } & \text { MASS GRAMS } \\ \text { CO2 } & \text { MASS GRAMS } \\ \text { NOX } & \text { MASS GRAMS } \\ \text { CH4 } & \text { MASS GRAMS } & \\ \text { NMIC } & \text { MASS GRAMS } & \text { (FID) } \\ \text { FUEL } & \text { MASS KG } & \end{array}$

FUEL ECONOMY MPG (I/10OKM)

$$
\begin{array}{r}
21.99 \\
130.07 \\
1948.41 \\
.3479 \\
1.90 \\
12.31 \\
116.29
\end{array}
$$

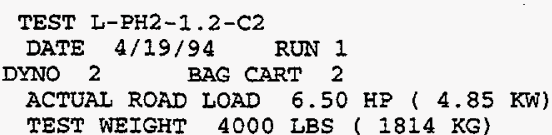

DRY BULB TEMPERATURE $72.0^{\circ} \mathrm{F}\left(22.2^{\circ} \mathrm{C}\right)$
COLD TRANSIEINT

(0-505 SEC.)

505.6

$.977 / .983$

$3.62(5.82)$

$558.2(15.81)$

$.27(.01)$

4706 . (133.3)

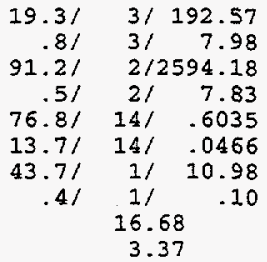

15.13

185.12

185.12
2504.90

.5600

10.88

13.53

169.96

14.753
388.639

388.639

1366.32

2.750

1.202

13.061
.657

$15.47(15.21)$
PROJECT NO. 08-6068-001

GASOLINE PHASE II EM-1701-E

FUEL DENSITY 6.186 IB/GAI

H $.139 \mathrm{C} .8410 .020 \mathrm{X} .000$

NOX HUMIDITY C.F. .991

HOT TRANSIENT

$(0-505$ SEC.)

505.5

$978, .983$

$3.62(5.83)$

$561.0(15.89)$

$.28(.01)$

4728 . (133.9)

$.28(.01)$

8092 . (229.2)

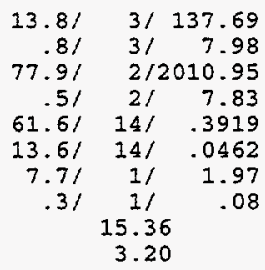

$15.4 / 3 / 153.66$

$8 / 3 / 7.98$

$94.7 / 2 / 2758.89$

$\begin{array}{lll}.41 & 21 & 6.26\end{array}$

$71.2 / 14 / \quad .5166$

$13.7 / 14 / 0466$

$19.4 / \quad 1 / 4.92$

$\begin{array}{lll}.5 / 1 / & 1 / & 4.92 \\ & 16\end{array}$

16.16

3.05

17.827

519.831

1459.54

.824

1. 881

15.367

.757

$14.43\{16.30\}$

16.54

146.16

2670.56

.4728

4.80

13.30

131.27

$$
\begin{array}{r}
11.767 \\
416.323 \\
1159.24 \\
1.219 \\
1.187 \\
10.136 \\
.500
\end{array}
$$

$16.92(13.90)$

3-BAG COMPOSITE RESULTS

$\begin{array}{llrrlrr}\text { THC } & \text { G/MI } & 4.110 & & \text { CH4 } & \text { G/MI } & .409 \\ \text { CO } & \text { G/MI } & 123.031 & & \text { NMHC } & \text { G/MI } & 3.562 \\ \text { NOX } & \text { G/MI } & .360 & & & \text { CARBONYL G/MI } & .067 \\ \text { FUEI ECONOMY MPG } & (\mathrm{L} / 100 \mathrm{KM}) & 15.27(15.41) & \text { AICOHOL G/MI } & .072\end{array}$




\section{APPENDIX F}

\section{COMPUTER PRINTOUTS OF EMISSIONS DATA WITH ETHANOL}

\begin{tabular}{|c|l|l|l||}
\hline Page F- & \multicolumn{1}{|c|}{ Test Number } & $\begin{array}{l}\text { Operating } \\
\text { Condition }\end{array}$ & \multicolumn{1}{|c|}{$\begin{array}{c}\text { Catalyst } \\
\text { Installation }\end{array}$} \\
\hline \hline 1 & L-ETH-0.8-E1 & Lean & Without Catalyst \\
\hline 2 & L-ETH-0.8-E2 & Lean & Without Catalyst \\
\hline 3 & L-ETH-0.8-C1 & Lean & With Catalyst \\
\hline 4 & L-ETH-0.8-C2 & Lean & With Catalyst \\
\hline \hline 5 & L-ETH-1.0-E1 & Stoich & Without Catalyst \\
\hline 6 & L-ETH-1.0-E2 & Stoich & Without Catalyst \\
\hline 7 & L-ETH-1.0-C2 & Stoich & With Catalyst \\
\hline 8 & L-ETH-1.0-C3 & Stoich & With Catalyst \\
\hline \hline 9 & L-ETH-1.2-E1 & Rich & Without Catalyst \\
\hline 10 & L-ETH-1.2-E2 & Rich & Without Catalyst \\
\hline 11 & L-ETH-1.2-C1 & Rich & With Catalyst \\
\hline 12 & L-ETH-1.2-C2 & Rich & With Catalyst \\
\hline
\end{tabular}


COMPUTER PROGRAM LDT $1.5-\mathrm{R}$

VEHICLE NUMBER 601

VEHICLE MODEL 88 CHEVY CORSICA

ENGINE

2.8 I ( 171 CID $)-V-6$

$\begin{array}{lc}\text { TRANSMISSION } & \text { MS } \\ \text { ODOMETER } & 14994 \text { MILES ( } 24125 \mathrm{KM})\end{array}$

BAROMETER 29.17 IN HG (340.9 MM HG)

RELATIVE HUMIDITY 54.4 PCT.

BAG NUMBER

BAG DESCRIPTION

RUN TIME SECONDS

DRY/WET CORRECTION FACTOR, SAMP/BACK

MEASURED DISTANCE MILES (KM)

BLOWER FLOW RATE SCFM (SCMM)

GAS METER FLOW RATE SCFM (SCMM)

TOTAI FLOW SCF (SCM)

HC SAMPLE METER/RANGE/PPM (BAG)

HC BCKGRD METER/RANGE/PPM

CO SAMPLE METER/RANGE/PPM

CO BCKGRD METER/RANGE/PPM

CO2 SAMPLE METER/RANGE/PCT

CO2 BCRGRD METER/RANGE/PCT

NOX SAMPLE METER/RANGE/PPM (BAG) (D)

NOX BCKGRD METER/RANGE/PPM

CH4 SAMPLE PPM $\{1.140)$

CH4 BCKGRD PPM

\section{DILUTION FACTOR}

HC CONCENTRATION PPM

CO CONCENTRATION PPM

$\mathrm{CO} 2$ CONCENTRATION PCT

NOX CONCENTRATION PPM

CH4 CONCENTRATION PPM

NMHC CONCENTRATION PPM

$\begin{array}{ll}\text { THE } & \text { MASS GRAMS } \\ \text { CO } & \text { MASS GRAMS } \\ \text { CO2 } & \text { MASS GRAMS } \\ \text { NOX } & \text { MASS GRAMS } \\ \text { CH4 } & \text { MASS GRAMS } \\ \text { NMHC } & \text { MASS GRAMS (FID) } \\ \text { FUEL } & \text { MASS KG }\end{array}$

FUEL ECONOMY MPG (L/100KM)

(BAG)

3-BAG COMPOSITE RESULTS

$\begin{array}{llr}\text { THC } & \text { G/MI } & 12.068 \\ \text { CO } & \text { G/MI } & 8.999 \\ \text { NOX } & \text { G/MI } & .608\end{array}$

FUEL ECONOMY MPG (I/100KM

$11.51(20.44)$

$$
\begin{aligned}
& \text { TEST C-ETH-0.8-E1 } \\
& \text { DATE } 6 / 30 / 94 \text { RUN }
\end{aligned}
$$
DYNO 2 BAG CART 2

ACTUAL ROAD LOAD $4.50 \mathrm{HP}(3.36 \mathrm{~kW})$ TEST WEIGET 3500 LBS (1587 KG)

DRY BULE TEMPERATURE $74.0^{\circ} \mathrm{F}\left(23.3^{\circ} \mathrm{C}\right)$

$$
\begin{gathered}
1 \\
\text { COID TRANSIENT } \\
(0-505 \text { SEC. }) \\
505.3 \\
.974(.984 \\
3.62(5.82) \\
556.6(15.76) \\
.27(1.01) \\
4690 .(132.8)
\end{gathered}
$$

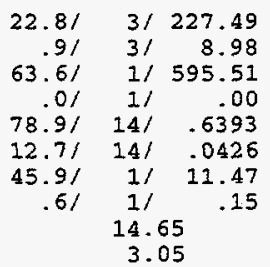

17.24

219.03

575.54

.5992

11.33

11.78

31.63

35.189

88.988

1456.91

2.813

1.043

2.422

$12.28(19.16)$

$$
\begin{aligned}
& 2 \\
& \text { STABILIZED } \\
& \text { (505-1372 SEC.) } \\
& 867.3 \\
& 976 / .984 \\
& 3.86(6.21) \\
& 557.2(15.78) \\
& .28(.01) \\
& 8058 .(228.2) \\
& 22.3 / 3 / 222.50 \\
& \begin{array}{rrr}
22.3 / & 3 / & 222.50 \\
.8 / & 3 / & 7.98
\end{array} \\
& \begin{array}{rrr}
79.8 / & 3 / & 7.98 \\
12 / & 78.37
\end{array} \\
& 1.0 / 12 / .95 \\
& 71.1 / 14 / 5152 \\
& 13.1 / 14 / \quad .0442 \\
& 14.4 / 1 / 3.60 \\
& \begin{array}{rrr}
4.4 / & 1 / & 3.60 \\
.2 / & 1 / & .05
\end{array} \\
& \begin{array}{l}
6.94 \\
3.07
\end{array} \\
& 22.85 \\
& 214.87 \\
& 75.09 \\
& .4729 \\
& 3.55 \\
& 4.01 \\
& 24.61 \\
& 1.113 \\
& 10.24(22.97)
\end{aligned}
$$

$\mathrm{CH} 4$

NMHC

G/MI

G/MI

CARBONYL G/MI ALCOHOL G/MI
RESEARCH

PROJECT NO. 08-6068-001

$$
\text { ETHANOL E100 EM-1803-F }
$$$$
\text { FUEI DENSITY } 6.514 \mathrm{LB} / \mathrm{GAI}
$$

$\begin{array}{llllllllll}H & \text { H } & 131 & \mathrm{C} & .521 & 0 & .347 & \mathrm{X} & .000\end{array}$

TEMP. FUEL FRACTIONS

NOX HUMIDITY C.F. $\quad .978$

3

HOT TRANSIENT

( 0 - 505 SEC.)

505.5

$.975 / .984$

$3.62(5.82)$

$556.1(15.75)$

$.27(.01)$

4688 . ( 132.8$)$

$\begin{array}{rrr}13.0 / & 3 / & 129.71 \\ .9 / & 3 / & 8.98 \\ 46.8 / & 13 / & 107.48 \\ .5 / & 13 / & 1.09 \\ 77.3 / & 14 / & .6118 \\ 13.3 / & 14 / & .0450 \\ 52.1 / & 1 / & 13.02 \\ .6 / & 1 / & .15 \\ & 6.47 & \\ 3.07 & \end{array}$

$$
\begin{array}{r}
19.49 \\
121.19 \\
102.93 \\
15692 \\
12.88 \\
3.56 \\
1.41
\end{array}
$$

22.577

15.908

1383.36

3.197

.315

.108

.760

$14.06(16.73)$ 
SOUTHWEST RESEARCH INSTITUTE - DEPARTMENT OF EMISSIONS RESEARCH

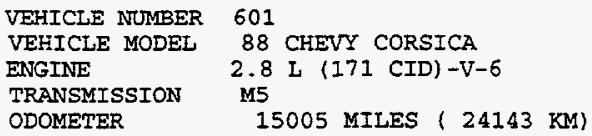

ODOMETER

BAROMETER 29.16 IN HG $(740.7 \mathrm{MM}$ HG) RELATIVE HUMIDITY 50.8 PCT.

BAG NUMBER

BAG DESCRIPTION

RUN TIME SECONDS

DRY/WET CORRECTION FACTOR, SAMP/BACK

MEASURED DISTANCE MIIES (KM)

BLOWER FLOW RATE SCFM (SCMM)

GAS METER FLOW RATE SCFM (SCMM)

TOTAL FLOW SCF (SCM)

HC SAMPLE METER/RANGE/PPM (BAG)

HC BCKGRD METER/RANGE/PPM

CO SAMPLE METER/RANGE/PPM

CO BCKGRD METER/RANGE/PPM

CO2 SAMPLE METER/RANGE/PCT

CO2 BCKGRD METER/RANGE/PCT

NOX SAMPLE METER/RANGE/PPM

NOX BCRGRD METER/RANGE/PPM

CH4 SAMPIE PPM $(1.140)$

CH4 BCKGRD PPM

DILUTION FACTOR

HC CONCENTRATION PPM

CO CONCENTRATION PPM

$\mathrm{CO} 2$ CONCENTRATION PCT

NOX CONCENTRATION PPM

CH4 CONCENTRATION PPM

NMHC CONCENTRATION PPM

$\begin{array}{ll}\text { THC } & \text { MASS GRAMS } \\ \text { CO } & \text { MASS GRAMS } \\ \text { CO2 } & \text { MASS GRAMS } \\ \text { NOX } & \text { MASS GRAMS } \\ \text { CH4 } & \text { MASS GRAMS } \\ \text { NMHC MASS GRAMS (FID) } & \\ \text { FUEL MASS KG }\end{array}$

FUEL ECONOMY MPG (L/100kM)

3-BAG COMPOSITE RESULTS

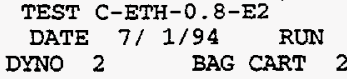

TEST C-ETH-0.8-E2

DATE $7 / 1 / 94$ RUN

DYNO 2 BAG CART

ACTUAI ROAD LOAD $4.50 \mathrm{HP}(3.36 \mathrm{~kW})$

TEST WEIGHT 3500 LBS ( $1587 \mathrm{KG})$

DRY BULB TEMPERATURE $74.0^{\circ} \mathrm{F}\left(23.3^{\circ} \mathrm{C}\right)$
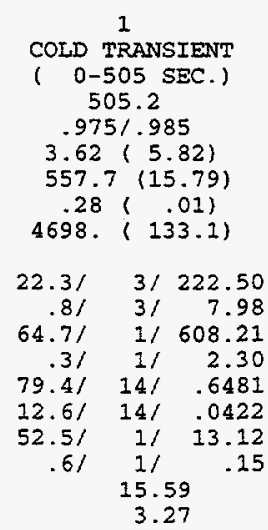

17.04

214.99

586.26

.6084

12.98

12.52

.22

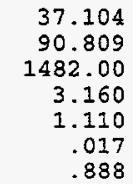

45.488

19.129

1950.83

1.467

.558

3.167

$10.57(22.26)$

$\mathrm{CH} 4$

NMHC

$G / M I$

ALCOHOL G/MI
$12.04 i(19.54)$

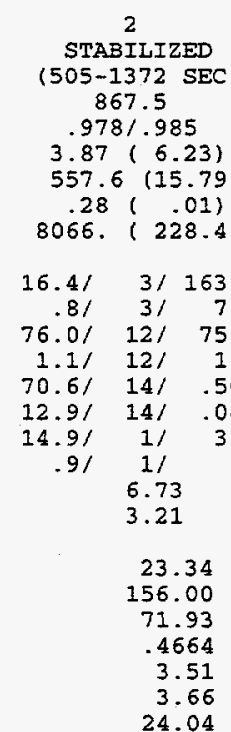

CARBONYL G/MI

PROJECT NO. 08-6068-001

ETHANOL E100 EM-1803-E FUET DENSTTY 6.514 TB/GAI

$\begin{array}{llllllll}\mathrm{H} & .131 & \mathrm{C} & .521 & 0 & .347 & \mathrm{X} & .000\end{array}$ TEMP. FUEI FRACTIONS

NOX HUMIDITY C.F. .957

$$
3
$$

HOT TRANSIENT

( $0-505$ SEC.)

505.4

$.976 / .985$

$3.62(5.82)$

$556.4(25.76)$

$.27(.01)$

$4689 .(132.8)$

$13.0 / 3 / 129.71$

$.8 / 3 / 7.98$

$49.2 / 13 / 113.36$

$.4 / 13 / \quad .87$

$77.3 / 14 / .6118$

$13.3 / 14 / \quad .0450$

$48.0 / 1 / 12.00$

$.8 / \quad 1 / \quad .20$

6.23

2.96

$$
\begin{array}{r}
19.46 \\
122.14 \\
108.96 \\
5692 \\
11.81 \\
3.43 \\
9.10
\end{array}
$$

21.605

16.845

1383.76

2.869

.303

.697

14.06 ( 16.73$)$

FUEL ECONOMY MPG (L/100KM) 11.67 (20.15)
.161
.477
1.388
7.825 
COMPUTER PROGRAM LDT $1.5-\mathrm{R}$

VEHICLE NUMBER 601

VEHICLE MODEI 88 CHEVY CORSICA

ENGINE

2.8 I ( 171 CID $)-V-6$

TRANSMISSION M5

ODOMETER 15023 MILES ( $24172 \mathrm{KM}$ )

BAROMETER 29.22 IN HG (742.2 MM HG)

RELATIVE HUMIDITY 60.4 PCT

BAG NUMBER

BAG DESCRIPTION

RUN TIME SECONDS

DRY/WET CORRECTION FACTOR, SAMP/BACK

MEASURED DISTANCE MILES (KM)

BLOWER FLOW RATE SCFM (SCMM)

GAS METER FLOW RATE SCFM (SCMM)

TOTAL FLOW SCF (SCM)

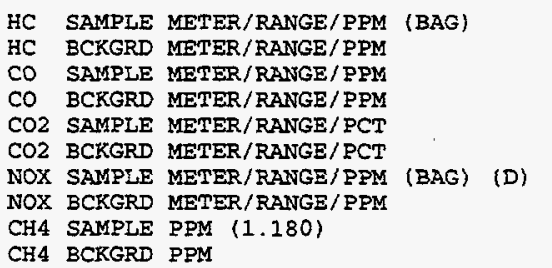

CH4 BCKGRD PPN

DILUTION FACTOR

HC CONCENTRATION PPM

CO CONCENTRATION PPM

$\mathrm{CO} 2$ CONCENTRATION PCT

NOX CONCENTRATION PPM

CH4 CONCENTRATION PPM

NMHC CONCENTRATION PPM

THC MASS GRAMS

CO MASS GRAMS

$\mathrm{CO} 2$ MASS GRAMS

NOX MASS GRAMS

CH4 MASS GRAMS

NMHC MASS GRAMS (FID)

FUEL MASS KG

FUEL ECONOMY MPG (L/100KM)

MA)

$$
\begin{gathered}
1 \\
\text { COLD TRANSIENT } \\
(0-505 \text { SEC. }) \\
505.7 \\
.973 / .984 \\
3.63(5.85) \\
558.3(15.81) \\
.28(1.01) \\
4708 .(133.3)
\end{gathered}
$$

DRY BULE TEMPERATURE $71.0^{\circ} \mathrm{F}\left(21.7^{\circ} \mathrm{C}\right)$

$$
\begin{aligned}
& \text { TEST C-ETH-0.8-C1 } \\
& \text { DATE } 7 / 5 / 94 \text { RUN }
\end{aligned}
$$$$
\text { DYNO } 2 \text { BAG CART } 2
$$

ACTUAL ROAD LOAD $4.50 \mathrm{HP}\{3.36 \mathrm{KW}\}$

TEST WEIGHT 3500 LBS ( $2587 \mathrm{KG}$ )

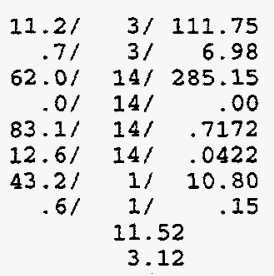

16.34

16.34
105.19

274.48

.6776

10.66

8.59

6.81

16.417

42.601

1653.85

2.657

.764

.523

$11.70(20.10)$

3-BAG COMPOSITE RESULTS

$\begin{array}{llr}\text { THC } & \text { G/MI } & 1.015 \\ \text { CO } & \text { G/MI } & 2.475 \\ \text { NOX } & \text { G/MI } & .548\end{array}$

FUEL ECONOMY MPG

$$
\begin{array}{r}
1.015 \\
2.475 \\
.548
\end{array}
$$

(L/100KM)

$11.12(21.15)$

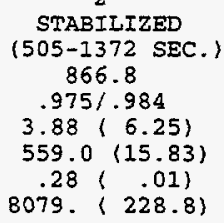

STABILIZED

(505-1372 SEC.) 866.8

$.975 / .984$

$3.88(6.25)$

$559.0(15.83)$

$.28(.01)$

B079. (228.8\}

$6.7 / 21 \quad 6.70$

$6.6121 \quad 6.60$

$1.1 / 12 / \cdot 1.04$

$.41 \quad 121 \quad .38$

$74.7 / \quad 14 / \quad .5695$

$13.1 / 14 / .0442$

$15.1 / 31 \quad 3.77$

$\begin{array}{lll}.8 / & 11 & .20 \\ & 3.19 & \end{array}$

3.09

21.56

.41
.65

$\begin{array}{r}.65 \\ .5273 \\ \hline\end{array}$

3.58

.24

$-1.32$

.439

2209.12

1.534

.036

.000
1.157

$9.92(23.71)$

$\begin{array}{lll}\text { CH4 } & \text { G/MI } & .059 \\ \text { NMHC } & \text { G/MI } & .030 \\ \text { CARBONYL } & \text { G/MI } & .061 \\ \text { ALCOHOL } & \text { G/MI } & .865\end{array}$

3
PROJECT NO. 08-6068-001

ETHANOL E100 EM-1803-F

FUEL DENSITY 6.514 LB/GAL

$\begin{array}{lllllllll}\text { H } & .131 & \mathrm{C} & .521 & 0 & .347 & \mathrm{X} & .000\end{array}$

TEMP. FUEL FRACTIONS

NOX HUMIDITY C.F. $\quad .978$

HOT TRANSIENT

$0-505$ SEC.

505.5

$.974 / .984$

$3.65(5.87)$

$557.9(15.80)$

$.27(.01)$

4703. (133.2)

$\begin{array}{rrr}7.4 / & 2 / & 7.40 \\ 5.9 / & 2 / & 5.90 \\ 1.8 / & 12 / & 1.71 \\ .4 / & 12 / & .38 \\ 80.1 / & 14 / & .6607 \\ 13.3 / & 14 / & .0450 \\ 41.1 / & 1 / & 10.27 \\ .4 / & 1 / & .10 \\ & 4.24 & \\ 2.89 & \end{array}$

18.58

1.82

1.30

6181

10.18

1.50

$-.54$

.230

1507.16

2.535

.134

.000

.789

$13.66(17.23)$ 
COMPUTER PROGRAM LDT $1.5-\mathrm{R}$

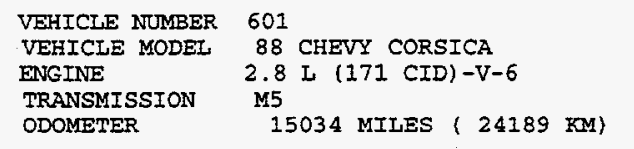

BAROMETER 29.18 IN HG (741.2 MM HG) RELATIVE HUMIDITY 60.4 PCT.

$$
\begin{aligned}
& \text { BAG NUMBER } \\
& \text { BAG DESCRIPTION }
\end{aligned}
$$

RUN TIME SECONDS

DRY/WET CORRECTION FACTOR, SAMP/BACK

MEASURED DISTANCE MILES (KM)

BLOWER FLOW RATE SCFM (SCMM)

GAS METER FLOW RATE SCFM (SCMM)

TOTAL FLOW SCF (SCM)

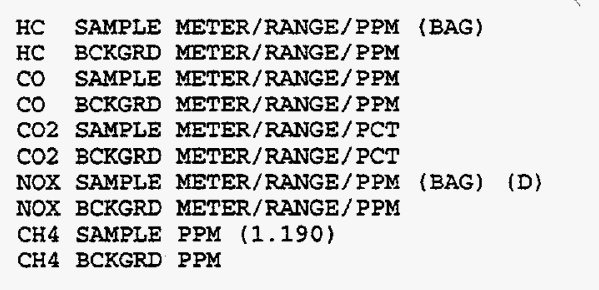

\section{DILUTION FACTOR}

HC CONCENTRATION PPM

CO CONCENTRATION PPM

$\mathrm{CO} 2$ CONCENTRATION PCT

NOX CONCENTRATION PPM

CH4 CONCENTRATION PPM

MAHC CONCENTRATION PPM

$\begin{array}{ll}\text { THC } & \text { MASS GRAMS } \\ \text { CO } & \text { MASS GRAMS } \\ \text { CO2 } & \text { MASS GRAMS } \\ \text { NOX } & \text { MASS GRAMS } \\ \text { CH4 } & \text { MASS GRAMS } \\ \text { MMHC } & \text { MASS GRAMS (FID) } \\ \text { FUEL } & \text { MASS KG }\end{array}$

FUEL ECONOMY MPG (L/100kM)

\section{3-BAG COMPOSITE RESULTS}

$\begin{array}{llr}\text { THC } & \text { G/MI } & .976 \\ \text { CO } & \text { G/MI } & 2.869 \\ \text { NOX } & \text { G/MI } & .600\end{array}$

FUEL ECONOMY MPG (I/100kM)

$11.40 \quad(20.64)$

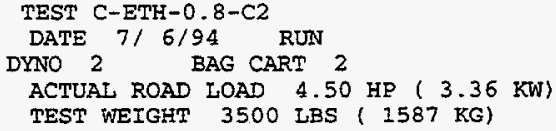

DRY BULB TEMPERATURE $71.0^{\circ} \mathrm{F}\left(21.7^{\circ} \mathrm{C}\right)$

$$
\begin{gathered}
1 \\
\text { COLD TRANSIENT } \\
(0-505 \text { SEC. }) \\
505.4 \\
.974 / .984 \\
3.63(5.84) \\
557.5(15.79) \\
.28(\quad .01) \\
4698 .(133.1)
\end{gathered}
$$

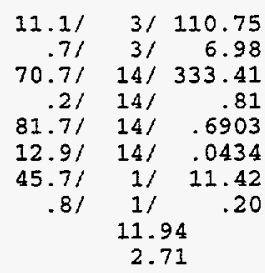

16.85

104.18

320.41

.6494

11.23

9.39
-.38

16.726

49.631

1582.05

2.798

.833

.000

$12.12(19.41)$

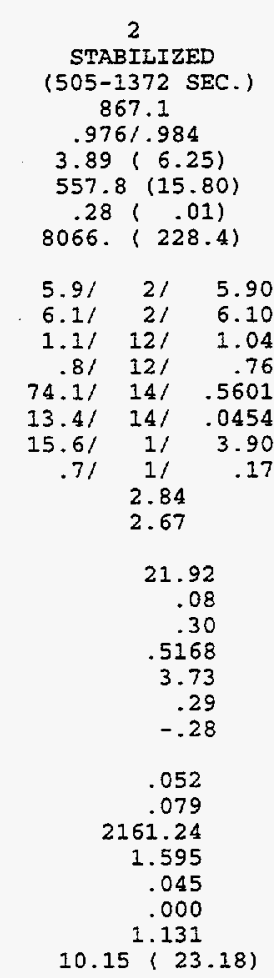

RESEARCH

PROJECT NO. 08-6068-001

ETHANOI EI00 EM-1803-F FUEI, DENSITY 6.514 IB/GAL

$\begin{array}{llllll}\mathrm{H} & .131 & \mathrm{C} & .521 & 0.347 & \mathrm{X} .000\end{array}$ TEMP. FUEL FRACTIONS

NOX HUMIDITY C.F. $\quad .979$

3

HOT TRANSIENT

( $0-505$ SEC.)

505.3

$.974 / .984$

$3.63(5.84)$

$557.6(15.79)$

$.27(.01)$

4699. (133.1)

$6.9 / 2 / 6.90$

$5.3 / 21 \quad 5.30$

$2.5 / 22 / 2.38$

$.7 / \quad 12 / \quad .66$

$\begin{array}{lll}79.3 / & 14 / & .6464\end{array}$

$13.5 / 14 / \quad 0458$

$49.1 / \quad 1 / \quad 12.27$

$\begin{array}{rrr}.91 & 11 & 12.27\end{array}$

3.84

19.00

1.88

1.68

.6030

12.06

1.47
-.09

.67

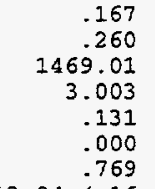

$13.94(16.87)$

$\begin{array}{lll}\text { CH4 } & \text { G/MI } & .063 \\ \text { NMAC } & \text { G/MI } & .000 \\ \text { CARBONYL } & \text { G/MI } & .059 \\ \text { AICOHOL } & \text { G/MI } & .854\end{array}$


COMPUTER PROGRAM LDT $1.5-R$

VEHICIE NUMBER 601

VEHICLE MODEL 88 CHEVY CORSICA

ENGINE

2.8 I (171 CID $)-V-6$

M5

14940 MILES ( $24038 \mathrm{kM}$ )

ODOMETER.

BAROMETER 29.18 IN HG (741.2 MM HG) RELATIVE HUMIDITY 57.0 PCT.

BAG NUMBER

BAG DESCRIPTION

RUN TIME SECONDS

DRY/WET CORRECTION FACTOR, SAMP/BACK

MEASURED DISTANCE MILES (KM)

BLOWER FLOW RATE SCFM (SCMM)

GAS METER FLOW RATE SCFM (SCMM)

TOTAL FLOW SCF (SCM)

HC SAMPLE METER/RANGE/PPM (BAG)

HC BCKGRD METER/RANGE/PPM

CO SAMPLE METER/RANGE/PPM

CO BCKGRD METER/RANGE/PPM

CO2 SAMPLE METER/RANGE/PCT

$\mathrm{CO} 2$ BCKGRD METER/RANGE/PCT

NOX SAMPLE METER/RANGE/PPM (BAG) (D)

NOX BCKGRD METER/RANGE/PPM

CH4 SAMPLE PDM (1.140)

CH4 BCKGRD PPM

\section{DILUTION FACTOR}

HC CONCENTRATION PPM

CO CONCENTRATION PPM

$\mathrm{CO} 2$ CONCENTRATION PCT

NOX CONCENTRATION PRM

CH4 CONCENTRATION PPM

MMHC CONCENTRATION PPM

THC MASS GRAMS

CO MASS GRAMS

$\mathrm{CO} 2$ MASS GRAMS

NOX MASS GRAMS

CH4 MASS GRAMS

NMHC MASS GRAMS (FID)

FUEL MASS KG

FUEL ECONOMY MPG (L/10OKM)

(BAG)

3-BAG CARB FTP VEHICLE EMISSION RESULTS

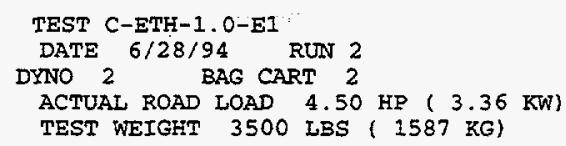

DRY BULB TEMPERATURE $72.0^{\circ} \mathrm{F}\left(22.2^{\circ} \mathrm{C}\right)$

$$
\begin{gathered}
1 \\
\text { COLD TRANSIENT } \\
(0-505 \text { SEC. } \\
505.7 \\
.975(.984 \\
3.61(5.81) \\
556.7(15.77) \\
.27(.01) \\
4694 .(132.9)
\end{gathered}
$$

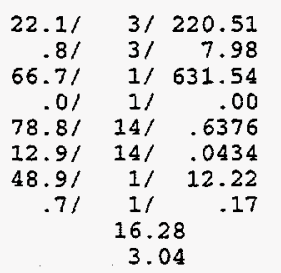

17.24

212.99

609.84

.5967

12.06

13.41

35.182

94.384

1452.27

2.977

1. 189

.356

$12.21(19.26)$

3-BAG COMPOSITE RESULTS
STABILIZED

(505-1372 SEC.) 867.4

$.977 / .984$

$3.91(6.29)$

$558.7(15.82)$

$.28(.01)$

8082 . (228.9)

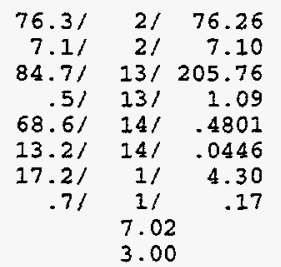

24.31

24.31
69.45

69.45
198.48

.4373

4.13

4.14

13.31

19.184

52.884

1832.53

1.756

.632

1.756

1. 022

$11.30(20.81)$

RESEARCH

PROJECT NO. 08-6068-001

ETHANOL EIOO EM-1803-F FUEL DENSITY $6.514 \mathrm{LB} / \mathrm{GAL}$

$\begin{array}{lllllll}\mathrm{H} & .131 & \mathrm{C} & .521 & 0.347 & \mathrm{X} & .000\end{array}$

TEMP. FUEL FRACTIONS

NOX HUMIDITY C.F. .971

$$
3
$$

HOT TRANSIENT

( $0-505$ SEC.

505.7

$.976 / .984$

$3.61(5.82)$

$558.1(15.81)$

$.27(.01)$

4706 . (133.3)

$\begin{array}{rrr}88.0 / & 2 / & 87.95 \\ 7.1 / & 2 / & 7.10 \\ 57.3 / & 14 / & 259.94 \\ .3 / & 14 / & 1.21 \\ 75.3 / & 14 / & .5790 \\ 13.4 / & 14 / & .0454 \\ 55.8 / & 1 / & 13.95 \\ .5 / & 1 / & .12 \\ & 7.36 & \\ 2.94 & \end{array}$

20.14

81.21

250.26

.5359

13.83

4.56

10.72

13.392

38.831

1307.65

3.423

.405

.824

$14.63(16.08)$

$\begin{array}{lrrrlll}\text { THC } & \text { G/MI } & 5.577 & & \text { CH4 } & \text { G/MI } & .183 \\ \text { CO } & \text { G/MI } & 15.370 & & \text { NMHC } & \text { G/MI } & .316 \\ \text { NOX } & \text { G/MI } & .663 & & & \text { CARBONYL G/MI } & .657 \\ & & & & \text { ALCOHOL G/MI } & 4.421 \\ \text { EUEL ECONOMY MPG } & (\mathrm{L} / 100 \mathrm{KM}) & 12.28(19.16) & & \end{array}$


SOUTHWEST RESEARCH INSTITUTE - DEPARTMENT OF EMISSIONS RESEARCH 3-BAG CARB FTP VEHICLE EMISSION RESULTS

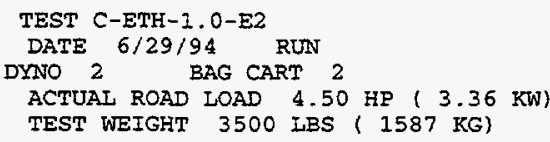

(BAG)
(BAG) (D)

$$
\begin{gathered}
1 \\
\text { COLD TRANSIENT } \\
(0-505 \text { SEC. } \\
505.0 \\
.974 / .984 \\
3.61(5.80) \\
558.9(15.83) \\
.28(1.01) \\
4706 .(133.3)
\end{gathered}
$$

31.309

90.167

1558.36

4.253

1.063

.807

.921

$11.57 ; 20.34)$
PROJECT NO. 08-6068-001

ETHANOL E100 EM-1803-F FUEI DENSITY $6.514 \mathrm{LB} / \mathrm{GAI}$

$\begin{array}{lllllll}\text { H } & .131 & \mathrm{C} .521 & 0.347 & \mathrm{X} .000\end{array}$ TEMP. FUEL FRACTIONS

NOX HUMIDITY C.F. .984

$$
\begin{aligned}
& 3 \\
& \text { HOT TRANSIENT } \\
& \text { ( 0- } 505 \text { SEC.) } \\
& 505.2 \\
& .975 / .984 \\
& 3.61(5.81) \\
& 559.2(15.84) \\
& .27(.01) \\
& \text { 4711. (133.4) } \\
& 75.3 / 2 / \quad 75.26 \\
& 9.1 / 2 / \quad 9.09 \\
& 58.4 / 14 / 265.78 \\
& .4 / 14 / \quad 1.62 \\
& 75.9 / 14 / \quad 5887 \\
& 13.3 / 14 / .0450 \\
& 62.3 / 1 / 15.57 \\
& 2.2 / \quad 1 / \quad .55 \\
& 7.27 \\
& 3.25 \\
& 66.62 \\
& 255.42 \\
& .5460 \\
& 15.05 \\
& 4.19 \\
& 2.40
\end{aligned}
$$

$\begin{array}{llr}\text { CH4 } & \text { G/MI } & .170 \\ \text { NMHC } & \text { G/MI } & .297 \\ \text { CARBONYL } & \text { G/MI } & .646 \\ \text { ALCOHOL } & \text { G/MI } & 3.801\end{array}$

$\begin{array}{llrlll}\text { THC } & \text { G/MI } & 4.914 & \text { CH4 } & \text { G/MI } & .170 \\ \text { CO } & \text { G/MI } & 15.960 & \text { NMHC } & \text { G/MI } & .297 \\ \text { NOX } & \text { G/MI } & .828 & \text { CARBONYL G/MI } & .646 \\ & & & \text { ALCOHOL G/MI } & 3.801\end{array}$


COMPUTER PROGRAM LDT 1.2-R

VEHICLE NUMBER 601

VEHICIE MODEL 88 CHEVY CORSICA

ENGINE $\quad 2.8$ I (171 CID) $-\mathrm{V}-6$

TRANSMISSION MS

14903 MILES ( $23978 \mathrm{kM}$ )

BAROMETER 29.06 IN HG (738.1 MM HG) RELATIVE HUMIDITY 56.5 PCT.

BAG NUMBER

BAG DESCRIPTION

RUN TIME SECONDS

DRY/WET CORRECTION FACTOR, SAMP/BACK

MEASURED DISTANCE MILES (KM)

BLOWER FLOW RATE SCFM (SCMM)

GAS METER FLOW RATE SCFM (SCMM)

TOTAL FLOW SCF (SCM)

HC SAMPLE METER/RANGE/PPM (BAG)

HC BCKGRD METER/RANGE/PPM

CO SAMPLE METER/RANGE/PPM

CO BCKGRD METER/RANGE/PPM

CO2 SAMPLE METER/RANGE/PCT

CO2 BCKGRD METER/RANGE/PCT

NOX SAMPLE METER/RANGE/PPM (BAG) (D)

NOX BCKGRD METER/RANGE/PPM

CH4 SAMPLE PPM (1.140)

CH4 BCKGRD PPM

DILUTION FACTOR

HC CONCENTRATION PPM

CO CONCENTRATION PPM

$\mathrm{CO} 2$ CONCENTRATION PCT

NOX CONCENTRATION PPM

CH4 CONCENTRATION PRM

NMIC CONCENTRATION PPM

THC MASS GRAMS

CO MASS GRAMS

$\mathrm{CO} 2$ MASS GRAMS

NOX MASS GRAMS

CH4 MASS GRAMS

NMTIC MASS GRAMS (FID)

FUEL MASS KG

FUEL ECONOMY MPG (L/100KM)

3-BAG COMPOSITE RESULTS

$\begin{array}{llr}\text { THC } & \text { G/MI } & .850 \\ \text { CO } & \text { G/MI } & 3.286 \\ \text { NOX } & \text { G/MI } & .557\end{array}$

FUEL ECONOMY MPG (L/100KM) $11.22 \quad$ (20.97)
3-BAG CARB INPTITUTE - DEPARTMENT OF EM

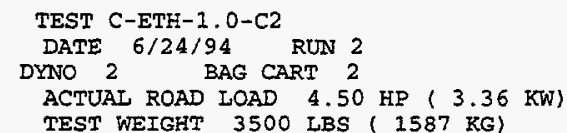

DRY BULB TEMPERATURE $71.0^{\circ} \mathrm{F}\left(21.7^{\circ} \mathrm{C}\right)$

COLD TRANSIEANT

$(0-505$ SEC. $)$

504.3

$.974 / .985$

$3.62(5.82)$

$557.8(15.80)$

$28(.01)$

4691 . (132.8)

$9.9 / 3 / 98.78$

$1.1 / 3 / 30.98$

$74.7 / 14 / 356.19$

$.6 / 214 / 2.43$

$83.7 / \quad 14 / \quad .7291$

$14.4 / \quad 14 / .0494$

$52.6 / 1 / 13.15$

$\begin{array}{llr}.7 / & 1 / & .17\end{array}$

13.59

4.13

$$
15.96
$$

88.49

340.97

.6828

12.98

9.72

14.217

52.730

1660.55

3.165

.861

.927

$11.54(20.39)$

STABILIZED

(505-1372 SEC.)

867.3

$.976 / .985$

$3.89 \cdot(6.26)$

$556.1(15.75\}$

$.28(.01)$

8042 . ( 227.8$)$

$\begin{array}{rrr}8.5 / & 2 / & 8.50 \\ 8.0 / & 2 / & 8.00 \\ 2.4 / & 12 / & 2.28 \\ 2.4 / & 12 / & 2.28 \\ 74.8 / & 14 / & .5711 \\ 14.8 / & 14 / & .0510 \\ 14.1 / & 1 / & 3.52 \\ .6 / & 1 / & .15 \\ & 4.27 & \\ & 4.13 & \end{array}$

21.49

.87

.07
.5224

5224
3.38

.33
.50

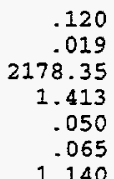

$10.08(23.35)$

$\begin{array}{lll}\text { CHA } & \text { G/MI } & .074 \\ \text { NMHC } & \text { G/MI } & .011 \\ \text { CARBONYL G/MI } & .062 \\ \text { ALCOHOL } & \text { G/MI } & .703\end{array}$

PROJECT NO. 08-6068-001

ETHANOL EI00 EM-1803-F FUEL DENSITY 6.514 LB/GAL

$\begin{array}{lllllllll}H & .131 & C & .521 & 0 & .347 & X & .000\end{array}$

TEMP. FUEL FRACTIONS

NOX HUMIDITY C.F. .960

HOT TRANSIENT

( $0-505$ SEC.

505.6

$.975 / .985$

$3.61(5.81)$

$554.0(15.69)$

$.27(.01)$

4671 . $(132.3)$

$10.1 / 2 / \quad 10.09$

$7.2121 \quad 7.20$

$26.0 / 12 / 25.15$

$2.01 \quad 12 / \quad 1.90$

$79.9 / 14 / \quad .6571$

$15.3 / 14 / .0531$

$42.0 / 1 / 10.50$

$\begin{array}{lll}1.3 / & 11 & .32\end{array}$

6.44
3.94

18.61

18.61
3.28

22.51

.6068

10.19

2.70

.20

.256

69.66

2.473

.238

.016
.772

$13.82(17.02)$ 
SOUTHWEST RESEARCH INSTITUTE - DEPARTMENT OF EMISSIONS RESEARCH

COMPUTER PROGRAM LDT I.5-R 3-BAG CARB FTP VEHICLE EMISSION RESULTS

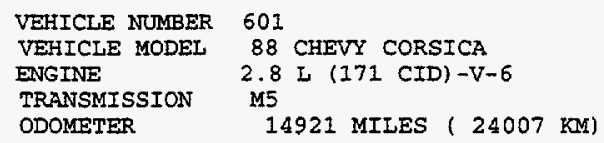

VEHICLE NUMBER 601

VEHICLE MODEL 88 CHEVY CORSICA

ENGINE

8 I (171 CID) $-\mathrm{V}-6$

14921 MIIES ( $24007 \mathrm{kN})$

BAROMETER 29.18 IN HG (741.2 MM HG) RELATIVE HUMIDITY 57.0 PCT.

BAG NUMBER

BAG DESCRIPTION

RUN TIME SECONDS

DRY/WET CORRECTION FACTOR, SAMP/BACK

MEASURED DISTANCE MILES (KM)

BLOWER FLOW RATE SCEM (SCMM)

GAS METER FLOW RATE SCFM (SCMM)

TOTAL FLOW SCF (SCM)

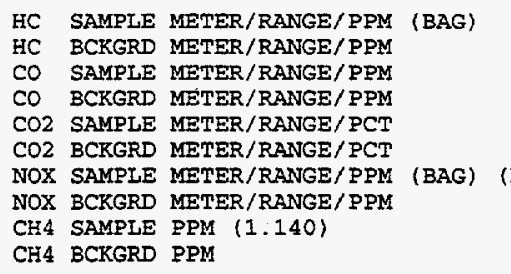

CH4 BCKGRD PPM

DILUTION FACTOR

HC CONCENTRATION PPM

CO CONCENTRATION PPM

$\mathrm{CO} 2$ CONCENTRATION PCT

NOX CONCENTRATION PPM

CH4 CONCENTRATION PPM

NMHC CONCENTRATION PPM

$\begin{array}{ll}\text { THC } & \text { MASS GRAMS } \\ \text { CO } & \text { MASS GRAMS } \\ \text { CO2 } & \text { MASS GRAMS } \\ \text { NOX } & \text { MASS GRAMS } \\ \text { CH4 } & \text { MASS GRAMS } \\ \text { NMHC } & \text { MASS GRAMS (FID) } \\ \text { FUEI } & \text { MASS KG }\end{array}$

FUEI, MASS KG

FUEI ECONOMY MPG (L/10OKM)

3-BAG COMPOSITE RESULTS

$$
\begin{aligned}
& \text { TEST C-ETH- } 1.0-C 3 \\
& \text { DATE } 6 / 27 / 94 \text { RUN } 2
\end{aligned}
$$$$
\text { DYNO } 2 \text { BAG CART } 2
$$

ACTUAL ROAD LOAD $4.50 \mathrm{HP}(3.36 \mathrm{KW}$ )

TEST WEIGHT 3500 LBS (1587 KG)

DRY BULB TEMPERATURE $72.0^{\circ} \mathrm{F}\left(22.2^{\circ} \mathrm{C}\right)$

$$
\begin{gathered}
1 \\
\text { COLD TRANSIENT } \\
(0-505 \text { SEC. }) \\
506.0 \\
.974 / .984 \\
3.62(5.82) \\
557.7(15.79) \\
.27(1.01) \\
4705 .(133.3)
\end{gathered}
$$

$12.2 / 3 / 121.73$

$\begin{array}{rrr}12.2 / & 3 / & 121.73 \\ .9 / & 3 / & 8.98\end{array}$

$86.01 \quad 14 / 421.87$

$.4 / 14 / 1.62$

$82.01 \quad 14 / .6960$

$13.7 / 14 / .0466$

$43.21 \quad 1 / \quad 10.80$

$\begin{array}{lll}.8 / & 1 / & .20\end{array}$

3.01

16.50

113.29

405.27

.6522

10.61

13.72

.72
.02

18.265

62.871

1591.16

2. 626

1.219

.002

$11.84(19.87)$

$$
\begin{aligned}
& \stackrel{2}{\text { STABILIZED }} \\
& \text { (505-1372 SEC.) } \\
& 867.1 \\
& .976 / .984 \\
& 3.87(6.23) \\
& 558.5(15.82) \\
& .28(.01) \\
& \text { 8076. (228.7) }
\end{aligned}
$$

$10.61(22.17)$

$\begin{array}{lll}\text { CH4 } & \text { G/MI } & .089 \\ \text { NMIC } & \text { G/MI } & .001 \\ \text { CARBONYL } & \text { G/MI } & .087 \\ \text { ALCOHOL } & \text { G/MI } & .894\end{array}$
PROJECT NO. 08-6068-001

ETHANOI E100 EM-1803-F FUEL DENSITY 6.514 LB/GAL

$\begin{array}{llllllll}\mathrm{H} & .131 & \mathrm{C} & .521 & 0.347 & \mathrm{X} & .000\end{array}$ TEMP. FUEL FRACTIONS

NOX HUMIDITY C.F. $\quad .971$

3

HOT TRANSIENT

( 0 - 505 SEC.)

506.1

$.975 / .984$

$3.63(5.84)$

558.2 (15.81)

$.27(.01)$

4711. (133.4)

$74.3 / \quad 1 / 7.45$

$55.9 / 1 / \quad 5.61$

$\begin{array}{lll}10.3 / & 121 & 9.87\end{array}$

$\begin{array}{lll}.81 / & 12 / & .625\end{array}$

$14.0 / 14 / .0478$

$45.2 / 1 / 11.30$

$.9 / 1 / \quad .22$

4.45

2.85

19.60

2.13

8.83

.5801

11.08

1.74
.17

.176

1.371

1416.85

2.746

.155

.013

.743

$14.43(16.30)$

$\begin{array}{llr}\text { THC } & \text { G/MI } & 1.071 \\ \text { CO } & \text { G/MI } & 3.720 \\ \text { NOX } & \text { G/MI } & .542\end{array}$

FUEL ECONOMY MFG (L/100KM)

$11.74(20.04)$ 
COMPUTER PROGRAM IDT 1.5-R

VEHICLE NUMBER 601

VEHICLE MODEL 88 CHEVY CORSICA

ENGINE

TRANSMISSION

ODOMETER

2.8 I $(171$ CID $)-V-6$

M5

15441 MILES ( $24844 \mathrm{KM}$ )

BAROMETER 29.16 IN HG (740.7 MM HG) RELATIVE HUMIDITY 61.0 PCT.

BAG NUMBER

BAG DESCRIPTION

RUN TIME SECONDS

DRY/WET CORRECTION FACTOR, SAMP/BACK

MEASURED DISTANCE MILES (KM)

BLOWER FLOW RATE SCFM (SCMM)

GAS METER FLOW RATE SCFM (SCMM)

TOTAI FLOW SCF (SCM)

HC SAMPIE METER/RANGE/PPM (BAG)

HC BCKGRD METER/RANGE/PPM

CO SAMPLE METER/RANGE/PPM

CO BCKGRD METER/RANGE/PPM

CO2 SAMPLE METER/RANGE/PCT

CO2 BCKGRD METER/RANGE/PCT

NOX SAMPLE METER/RANGE/PPM (BAG)

NOX BCRGRD METER/RANGE/PPM

CH4 SAMPLE PPM $(1.150)$

CH4 BCKGRD PPM

DILUTION FACTOR

HC CONCENTRATION PPM

CO CONCENTRATION PPM

$\mathrm{CO} 2$ CONCENTRATION PCT

NOX CONCENTRATION PPM

CH4 CONCENTRATION PPM

NMHC CONCENTRATION PRM

THC MASS GRAMS

CO MASS GRAMS

$\mathrm{CO} 2$ MASS GRAMS

NOX MASS GRAMS

CH4 MASS GRAMS

NMHC MASS GRAMS (FID)

FUEL MASS KG

FUEL ECONOMY MPG (L/100KM)

3-BAG COMPOSITE RESULTS

$\begin{array}{llr}\text { THC } & \text { G/MI } & 6.130 \\ \text { CO } & \text { G/MI } & 22.636 \\ \text { NOX } & \text { G/MI } & .930\end{array}$

FUEL ECONOMY MPG (L/100KM) 11.10 (21.19)

$$
\begin{gathered}
1 \\
\text { COLD TRANSIENT } \\
(0-505 \text { SEC. }) \\
505.1 \\
.973 / .983 \\
3.60(5.80) \\
554.6(15.71) \\
.28(.01) \\
4671 .(132.3)
\end{gathered}
$$

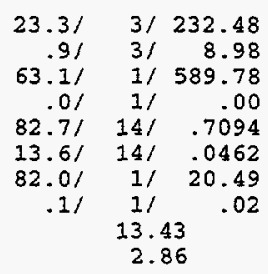

15.73

224.07

567.71

.6662

20.47

10.76

1.96

37.219

87.429

1613.39

5.143

.949

.150

.954

$11.16(21.08)$
3-BAG CARB FTP VEHICLE EMISSION RESULTS

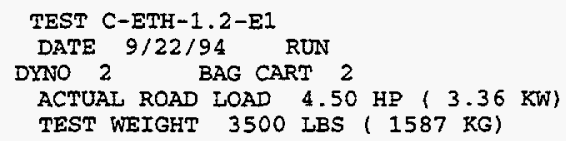

DRY BULB TEMPERATURE $72.0^{\circ} \mathrm{F}\left(22.2^{\circ} \mathrm{C}\right)$
2
STABILIZED
(505-1372 SEC.) 867.4
$975 / .983$
$3.86(6.21)$
$557.6(15.79)$
$28(.01)$
8065 . (228.4)

$78.8 / 2 / \quad 78.75$

$6.4 / 21 \quad 6.40$

$79.9 / \quad 14 / 386.24$

$.5 / 14 / \quad 2.02$

$71.5 / 14 / .5210$

$13.6 / 14 / .0462$

$28.7 / 1 / 7.17$

$.011 / .00$

7.90

2.75

$$
\begin{array}{r}
21.80 \\
72.65 \\
371.71 \\
.4769 \\
7.17 \\
5.28 \\
10.23
\end{array}
$$

19.942

98.845

1994.37

3.112

.803

1.347

1.145

$9.96(23.63)$

RESEARCH

PROJECT NO. 08-6068-001

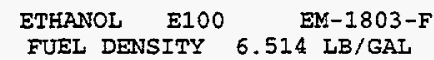

H .131 C . $5210.347 \mathrm{X} .000$

NOX HUMIDITY C.F. .993

3

HOT TRANSIENT

( $0-505$ SEC.)

504.4

$.974 / .983$

$3.61(5.82)$

$556.3(15.76)$

$.28(.01)$

4679. ( 132.5$)$

$11.6 / 3 / 115.74$

$8 / 3 / 7.98$

$79.8 / \quad 14 / 385.65$

$.4 / 14 / \quad 1.62$

$76.3 / 14 / .5952$

$13.3 / 14 / .0450$

$45.2 / \mathrm{I} / 11.30$

$.01 \quad 1 / \quad .00$

9.48

2.52

19.19

108.18

370.82

.5526

11.30

7.09

15.56

17.243

57.207

1340.63

2.843

.627

1. 189

.766

$13.94(16.87)$ $\begin{array}{ll}\text { CH4 } & \text { G/MI } \\ \text { MMIC } & \text { G/MI } \\ \text { CARBONIL } & \text { G/MI } \\ \text { ALCOHOL } & \text { G/MI }\end{array}$
.210
.280
.639
5.001 
SOUTHWEST RESEARCH INSTITUTE - DEPARTMENT OF EMISSIONS RESEARCH 3-BAG CARB FTP VEHICLE EMISSION RESULTS

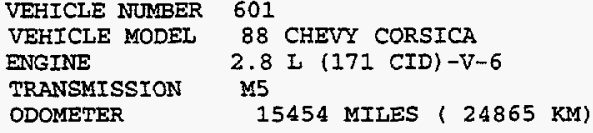

BAROMETER 29.24 IN HG $(742.7 \mathrm{MM} \mathrm{HG})$ RELATIVE HUNIDITY 42.7 PCT.

BAG NUMBER

BAG DESCRIPTION

RUN TIME SECONDS

DRY/WET CORRECTION FACTOR, SAMP/BACK

MEASURED DISTANCE MILES (KM)

BLOWER FLOW RATE SCFM (SCMM)

GAS METER FLOW RATE SCFM (SCMM)

TOTAL FLOW SCF (SCM)

HC SAMPLE METER/RANGE/PPM (BAG)

HC BCKGRD METER/RANGE/PRM

CO SAMPLE METER/RANGE/PPM

CO BCKGRD METER/RANGE/PEM

CO2 SAMPLE METER/RANGE/PCT

CO2 BCKGRD METER/RANGE/PCT

NOX SAMPLE METER/RANGE/PPM

NOX BCKGRD METER/RANGE/PRM

CH4 SAMPLE PPM (1.150)

CH4 BCKGRD PPM

DILUTION FACTOR

HC CONCENTRRATION PPM

CO CONCENTRATION PEM

$\mathrm{CO} 2$ CONCENTRATION PCT

NOX CONCENTRATION PPM

CH4 CONCENTRATION PPM

NMAC CONCENTRATION PPM

$\begin{array}{ll}\text { THC } & \text { MASS GRAMS } \\ \text { CO } & \text { MASS GRAMS } \\ \text { CO2 } & \text { MASS GRAMS } \\ \text { NOX } & \text { MASS GRAMS } \\ \text { CH4 } & \text { MASS GRAMS } \\ \text { NMHC } & \text { MASS GRAMS (FID) } \\ \text { FUEL } & \text { MASS KG }\end{array}$

FUEI (I/ $/ 100 \mathrm{KM}$ )

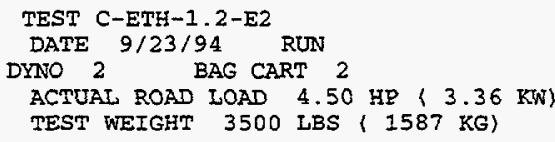

DRY BULE TEMPERATURE $68.0^{\circ} \mathrm{F}\left(20.0^{\circ} \mathrm{C}\right)$

$$
\begin{gathered}
1 \\
\text { COLD TRANSIENT } \\
(0-505 \text { SEC. }) \\
505.4 \\
.979 / .990 \\
3.60(5.79) \\
557.5(15.79) \\
.28(.01) \\
4698 .(133.0)
\end{gathered}
$$

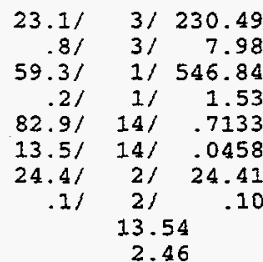

15.73

223.01

528.13

.6704

24.32

11.23

12.10

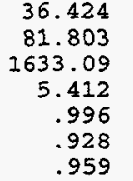

$11.09 ; 21.21)$

$$
\begin{aligned}
& 2 \\
& \text { STABILIZE } \\
& \text { (505-1372 SEC.) } \\
& 867.0 \\
& .9821 .990 \\
& 3.88(6.24) \\
& 559.5(15.85) \\
& .28 \text { (. .01) } \\
& 8089 .(229.1) \\
& \begin{array}{rrr}
71.9 / & 2 / & 71.86 \\
6.4 / & 2 / & 6.40
\end{array} \\
& \begin{array}{rrr}
6.4 / & 2 / & 6.40
\end{array} \\
& 71.6 / 14 / 338.51 \\
& .8 / 14 / 3.23 \\
& 72.0 / \quad 14 / \quad .5283 \\
& 13.6 / 14 / \quad .0462 \\
& 40.9 / 1 / 10.22 \\
& .811 \\
& 6.81 \\
& 2.59 \\
& 21.70 \\
& 65.76 \\
& 326.33 \\
& .4843 \\
& 10.03 \\
& 4.34 \\
& 13.97 \\
& 17.689 \\
& 87.028 \\
& 2031.00 \\
& 3.844 \\
& .662 \\
& \text { 1. } 845 \\
& 1.152 \\
& 9.94(23.66)
\end{aligned}
$$

PROJECT NO. 08-6068-001

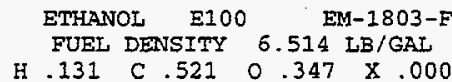

NOX HUMIDITY C.F. .875 HOT TRANSIENT

$(0-505$ SEC. $)$

$$
505.2
$$$$
.981 / .990
$$

$3.61(5.81)$

$558.5(15.82)$

$.27(.01)$

$4705 .(133.2)$

$9.8 / 3 / 97.78$

$\begin{array}{rrr}.7 / & 3 / & 6.98\end{array}$

$74.3 / 14 / 353.90$

$.6 / 24 / 2.43$

$\begin{array}{llll}75.7 / & 14 / \quad .5855\end{array}$

$12.7 / 14 / \quad .0426$

$51.8 / 1 / 12.95$

$.9 / \begin{array}{rr}1 / & .22\end{array}$

2.22

19.61
91.15
341.57
.5450
12.73
5.54
14.17

14.816

52.981

1329.44

2.838

492

1.089
.754

$14.15\langle 16.63)$

3-BAG COMPOSITE RESULTS

$\begin{array}{llr}\text { THC } & \text { G/MI } & 5.586 \\ \text { CO } & \text { G/MI } & 20.365 \\ \text { NOX } & \text { G/MI } & 1.041\end{array}$

$\begin{array}{ll}\text { CH4 } & \text { G/MI } \\ \text { NMHC } & \text { G/MI } \\ \text { CARBONYL } & \text { G/MI }\end{array}$

.183

.633

4.387 
COMPUTER PROGRAM LDT 1.5-R

VEHICLE NUMBER 601

VEFICLE MODEL 88 CHEVY CORSICA

ENGINE

2.8 I (171 CID) $-\mathrm{V}-6$

15473 MILES ( $24896 \mathrm{KM}$ )

BAROMETER 29.22 IN HG $(742.2 \mathrm{MM} \mathrm{HG})$ RELATIVE HUMIDITY 45.0 PCT

BAG NUMBER

BAG DESCRIPTION

RUN TIME SECONDS

DRY/WET CORRECTION FACTOR, SAMP/BACK

MEASURED DISTANCE MILES (KM)

BLOWER FLOW RATE SCFM (SCMM)

GAS METER FLOW RATE SCFM (SCMM)

TOTAL FLOW SCF (SCM)

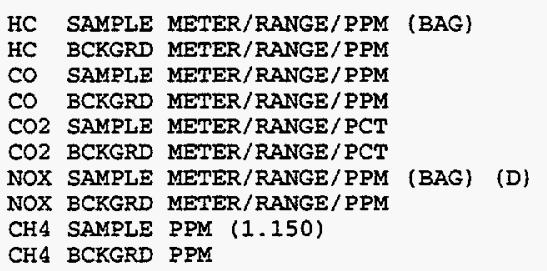

CHA BCKGRD PPM

DILUTION FACTOR

HC CONCENTRATION PPM

CO CONCENTRATION PPM

$\mathrm{CO} 2$ CONCENTRATION PCT

NOX CONCENTRATION PPM

CH4 CONCENTRATION PPM

NMIIC CONCENTRATION PPM

$\begin{array}{lll}\text { THC } & \text { MASS GRAMS } \\ \text { CO } & \text { MASS GRAMS } \\ \text { CO2 } & \text { MASS GRAMS } \\ \text { NOX } & \text { MASS GRAMS } \\ \text { CH4 } & \text { MASS GRAMS } \\ \text { NMHC } & \text { MASS GRAMS (FID) } \\ \text { FUEL } & \text { MASS KG } & \end{array}$

FUEL ECONOMY MPG (L/100kM

3-BAG COMPOSITE RESULTS

$\begin{array}{llr}\text { THC } & \text { G/MI } & 1.198 \\ \text { CO } & \text { G/MI } & 3.582 \\ \text { NOX } & \text { G/MI } & .417\end{array}$

FUEL ECONOMY MPG (I/100KM)

$10.37 \quad(22.69)$

$$
\begin{gathered}
1 \\
\text { COLD TRANSIENT } \\
(0-505 \text { SEC. }) \\
505.8 \\
.976 / .988 \\
3.59(5.78) \\
562.2(15.92) \\
.28(.01) \\
4742 .(134.3)
\end{gathered}
$$

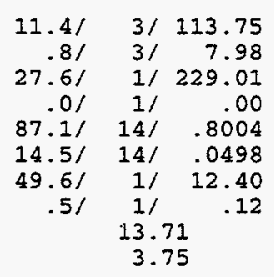

14.81

14.81
106.30

206.30
221.10

.7539
1.12

12.28

10.22

1.98
1.98

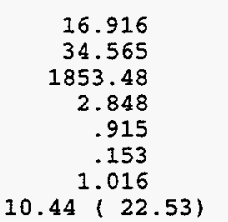

$10.44(22.53)$
TEST C-ETH-1 2-C1

DATE $9 / 26 / 94$ RUN

DYNO 2 BAG CART 2

ACTUAL ROAD LOAD $4.50 \mathrm{HP}$ ( $3.36 \mathrm{KW})$

TEST WEIGHT 3500 LBS ( $1587 \mathrm{KG}$ )

DRY BULB TEMPERATURE $71.0^{\circ} \mathrm{F}\left(21.7^{\circ} \mathrm{C}\right)$

$$
\begin{aligned}
& \text { STABILIZED } \\
& \text { (505-1372 SEC.) } \\
& 867.2 \\
& .979 / .988 \\
& 3.88(6.24) \\
& 562.5(15.93) \\
& .28(.01) \\
& 8133 .(230.3) \\
& \begin{array}{lll}
7.8 / 21 & 7.80
\end{array} \\
& \begin{array}{lll}
7.87 & 27 & 7.80 \\
6.81 & 21 & 6.80
\end{array} \\
& \begin{array}{rrr}
6.8 / & 2 / & 6.80 \\
11.5 / & 12 / & 11.32
\end{array} \\
& 2.2 / 12 / \quad 2.18 \\
& 76.4 / 14 / \quad .5969 \\
& 14.7 / 14 / .0506 \\
& 11.1 / 1 / 2.77 \\
& \begin{array}{lll}
.31 & 11 & .07
\end{array} \\
& \text { 3. } 53 \\
& 20.53 \\
& \begin{array}{r}
20.53 \\
1.33
\end{array} \\
& 8.93 \\
& .5487 \\
& 2.70 \\
& .99 \\
& .189 \\
& 2.396 \\
& 2313.95 \\
& 1.075 \\
& .152 \\
& .035 \\
& \text { 1. } 213 \\
& 9.45(24.89)
\end{aligned}
$$

RESEARCH

PROJECT NO. 08-6068-001

$$
\begin{aligned}
& \text { ETHANOL E100 } \\
& \text { FUEL DENSITY } 6.514 \mathrm{LB} / \mathrm{GAL}
\end{aligned}
$$$$
\begin{array}{llllllll}
\text { H. } & .131 & \mathrm{C} & .521 & 0 & 0.347 & \mathrm{X} & .000
\end{array}
$$

NOX HUMIDITY C.F. .903

$$
3
$$

HOT TRANSIENT

( $0-505$ SEC.)

505.0

$.978 / .988$

$3.56(5.74)$

$561.5(15.90)$

$.27(.01)$

4728. ( 133.9$)$

$27.4 / 2 / 27.38$

$6.6 / 2 / \quad 6.60$

$48.9 / 13 / 113.50$

$1.6 / 13 / 3.68$

$81.5 / 14 / .6865$

$14.2 / 14 / .0486$

$24.9 /$ if 6.22

.21 1/ $\quad .05$

6.80

2.95

17.56

17.56
21.16

21.16
106.49

.6407

6.18

4.02
12.24

2.601

16.601

1570.66

1. 428

.359

.945
.838

$12.56(18.73)$

$\begin{array}{lll}\text { CH4 } & \text { G/MI } & .101 \\ \text { NMHC } & \text { G/MI } & .086 \\ \text { CARBONYL } & \text { G/MI } & .102 \\ \text { ALCOHOL } & \text { G/MI } & .910\end{array}$ 
SOUTHWEST RESEARCH INSTITUTE - DEPARTMENT OF EMISSIONS RESEARCH 3-BAG CARB ETP VEHICLE EMISSION RESULTS

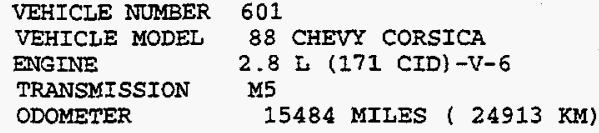

BAROMETER 29.16 IN HG $(740.7 \mathrm{MG}$ ) RELATIVE HUMIDITY 46.5 PCT.

BAG NUMBER

BAG DESCRIPTION

RUN TIME SECONDS

DRY/WET CORRECTION FACTOR, SAMP/BACK

MEASURED DISTANCE MILES (KM)

BLOWER FLOW RATE SCFM (SCMM)

GAS METER FLOW RATE SCFM (SCMM)

TOTAL FLOW SCF (SCM)

HC SAMPLE METER/RANGE/PPM (BAG)

HC BCKGRD METER/RANGE/PPM

CO SAMPLE METER/RANGE/PPM

CO BCKGRD METER/RANGE/PPM

$\mathrm{CO} 2$ SAMPLE METER/RANGE/PCT

CO2 BCKGRD METER/RANGE/PCT

NOX SAMPLE METER/RANGE/PPM

NOX BCKGRD METER/RANGE/PPM

CH4 SAMPLE PPM $(1.150)$

CH4 BCKGRD PQM

\section{DILUTION FACTOR}

HC CONCENTRATION PRM

CO CONCENTRATION PEM

$\mathrm{CO} 2$ CONCENTRATION PCT

NOX CONCENTRATION PPK

CH4 CONCENTRATION PPM

NMHC CONCENTRATION PPM

$\begin{array}{lll}\text { THC } & \text { MASS GRAMS } \\ \text { CO } & \text { MASS GRAMS } \\ \text { CO2 } & \text { MASS GRAMS } \\ \text { NOX } & \text { MASS GRAMS } \\ \text { CH4 } & \text { MASS GRAMS } \\ \text { NMHC } & \text { MASS GRAMS } & \text { (FID) } \\ \text { FUEI } & \text { MASS KG } & \end{array}$

FUEL ECONOMY MPG (L/10OKM)

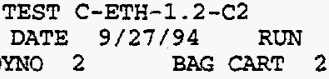

$$
\begin{gathered}
1 \\
\text { COID TRANSIENT } \\
(0-505 \text { SEC. }) \\
505.3 \\
.976(.987 \\
3.59(5.78) \\
562.8(15.94) \\
.28(.01) \\
4742 .(134.3)
\end{gathered}
$$

$15.9 /$

$1.0 \%$

3/ 158.65

$3.013 / 9.98$

$2 /$ I/ 1.53

$8.5 / 14 / .7452$

$\begin{array}{lll}84.5 / & 14 / & .7452 \\ 13.6 / & 14 / & .0452\end{array}$

$\begin{array}{rrr}13.6 / & 14 / & .0452 \\ 34.9 / & 1 / & 8.72\end{array}$

(BAG) (D)

.61

23.09

3.83

15.32

149.32

460.62

.7021

8.58

19.51

11.49

22.312

72.016

1726.22

2.040

1.747

.890

985

$10.77 ; 21.85)$

8.72
.15

2

$$
\begin{gathered}
2 \\
\text { STABIIIZED } \\
(505-1372 \text { SEC. }) \\
867.3 \\
.978 / .987 \\
3.88(6.24) \\
566.2(16.03) \\
.28\left(\begin{array}{c}
.01) \\
8188 .(231.9)
\end{array}\right.
\end{gathered}
$$

4.19

2.76

21.01

1.82

19.02

.5409

2.10

1.56
.03

.251
5.134

2296.35

.863

.241

.004
ACTUAL ROAD IOAD $4.50 \mathrm{HP}(3.36 \mathrm{~kW})$

TEST WEIGHT 3500 LBS ( $1587 \mathrm{KG}$ )

DRY BULB TEMPERATURE $73.0^{\circ} \mathrm{F}\left(22.8^{\circ} \mathrm{C}\right)$

$\begin{array}{lll}8.21 & 21 & 8.20 \\ 6.71 & 21 & 6.70\end{array}$

$21.5 / 12 / 21.04$

$1.5 / 12 / 1.49$

$75.5 / 14 / .5822$

$12.9 / 14 / \quad .0434$

$8.8 / 1 / 2.20$

$.41 \quad 1 / \quad .10$

$9.50(24.77)$

PROJECT NO. 08-6068-001

ETHANOL E100 EM-1803-F

FUEL DENSTTY 6.514 IB/GAI

H $.131 \subset .5210 .347 \quad \times .000$

NOX HUMIDITY C.F. .925

3

HOT TRANSIENT

( 0 - 505 SEC.

505.2

$.977 / .987$

$3.63(5.83)$

$563.1(15.95)$

$27(.01)$

4744. (134.3)

$\begin{array}{rrr}19.4 / & 2 / & 19.39 \\ 6.2 / & 2 / & 6.20 \\ 95.5 / & 12 / & 95.82 \\ 1.7 / & 12 / & 1.69 \\ 80.8 / & 14 / & .6735 \\ 13.1 / & 14 / & .0442 \\ 32.8 / & 1 / & 8.20 \\ .5 / & 1 / & .12 \\ 11.47 & \\ 2.53 & \end{array}$

17.96

13.54

91.20

.6317

8.08

9.08

2.59

1.126

14.262

1553.77

1. 921

813

201

$12.97\left(\begin{array}{l}.826 \\ (14)\end{array}\right.$
.195

.067

.067

1.075

FUEL ECONOMY MPG (L/100KM) 10.55 (22.30) 


\section{APPENDIX G}

\section{COMPUTER PRINTOUTS OF EMISSIONS DATA WITH METHANOL}

\begin{tabular}{|c|l|l|l||}
\hline Page G- & \multicolumn{1}{|c|}{ Test Number } & $\begin{array}{c}\text { Operating } \\
\text { Condition }\end{array}$ & \multicolumn{1}{|c|}{$\begin{array}{c}\text { Catalyst } \\
\text { Installation }\end{array}$} \\
\hline \hline 1 & L-MTH-0.8-E1 & Lean & Without Catalyst \\
\hline 2 & L-MTH-0.8-E3 & Lean & Without Catalyst \\
\hline 3 & L-MTH-0.8-C1 & Lean & With Catalyst \\
\hline 4 & L-MTH-0.8-C2 & Lean & With Catalyst \\
\hline \hline 5 & L-MTH-1.0-E1 & Stoich & Without Catalyst \\
\hline 6 & L-MTH-1.0-E2 & Stoich & Without Catalyst \\
\hline 7 & L-MTH-1.0-C2 & Stoich & With Catalyst \\
\hline 8 & L-MTH-1.0-C2 & Stoich & With Catalyst \\
\hline \hline 9 & L-MTH-1.2-E1 & Rich & Without Catalyst \\
\hline 10 & L-MTH-1.2-E2 & Rich & Without Catalyst \\
\hline 11 & L-MTH-1.2-C2 & Rich & With Catalyst \\
\hline 12 & L-MTH-1.2-C3 & Rich & With Catalyst \\
\hline
\end{tabular}


SOUTHWEST RESEARCH INSTITUTE - DERARTMENT OF EMISSIONS RESEARCH

VEHICLE MODEI 88 CHEVY CORSICA

ENGINE

2.8 I (171 CID) $-\mathrm{V}-6$
COMPUTER PROGRAM LDT $1.5-R$

VEHTCIE NUMBER 601

15233 MILES ( $24509 \mathrm{KM})$

3-BAG CARB FTP VEHICLE EMISSION RESULTS

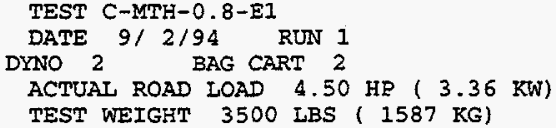

RAROMETER 29.35 IN HG (745.5 MM HG) RELATIVE HUMIDITY 58.6 PCT.

BAG NUMBER

BAG DESCRIPTION

RUN TIME SECONDS

DRY/WET CORRECTION FACTOR, SAMP/BACK

MEASURED DISTANCE MILES (IOM)

BLOWER FLOW RATE SCFM (SCMM)

GAS METER FLOW RATE SCFM (SCMM)

TOTAL FLOW SCF (SCM)

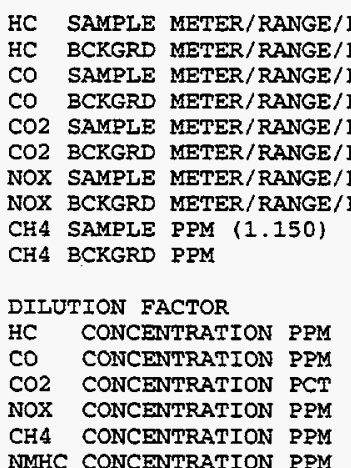

(BAG)

$\begin{array}{rrr}12.1 / & 3 / & 120.73 \\ .8 / & 3 / & 7.98\end{array}$

$74.4 / 14 / .354 .47$

$.4 / 14 / 1.62$

$77.5 / 14 / .6152$

$13.2 / \quad 14 / \quad .0446$

$40.6 / 1 / 10.15$

$1.0 / 1 / \quad .25$

4.66

3.65

17.66

113.20

339.83

.5731

9.91

1.22
-80.01

45.540

53.182

1410.59

2.581

.109

.000

1.133

$9.57(24.57)$

$75.0^{\circ} \mathrm{F}\left(23.9^{\circ} \mathrm{C}\right)$

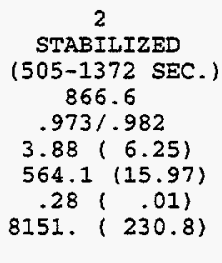

$\begin{array}{rrr}11.2 / & 3 / & 111.75 \\ .9 / & 3 / & 8.98 \\ 58.1 / & 13 / & 136.45 \\ .7 / & 13 / & 1.61 \\ 67.2 / & 14 / & .4613 \\ 13.5 / & 14 / & .0458 \\ 14.3 / & 1 / & 3.57 \\ .5 / & 1 / & .12 \\ & 4.00 & \\ & 3.72 & \end{array}$

24.13

103.14

130.49

.4174

. 3.45

22.55

37.723
35.067
1764.15
1.545
.067
3.002
1.362
$.54(27.53)$

RESEARCH
PROJECT NO. 08-6068-001

METHANOL EM-1791-F

FUEL DENSITY 6.610 I.B/GAL

H .123 C . $.3750 .502 \quad \mathrm{X} .000$

NOX HUMIDITY C.F. 1.013

3

HOT TRANSIENT

( 0 - 505 SEC.)

504.6

$.972 / .982$

$3.58(5.76)$

$562.3(15.93)$

$.28(.01)$

4732. (134.0)

$9.9 / 3 / 98.78$

$.9 / 3 / 8.98$

$67.2 / 13 / 159.81$

.7/ $13 / 1.61$

$73.2 / \quad 14 / \quad .5463$

$\begin{array}{lll}14.0 / 14 / & .0478\end{array}$

$38.4 / 1 / 9.60$

$.6 / 1 / \quad .15$

3.94

3.67

$$
\begin{array}{r}
20.50 \\
90.24 \\
152.71 \\
.5008 \\
9.45 \\
.45 \\
-7.27
\end{array}
$$

23.717

23.822

1228.66

2.454

.040

.000

$11.36(20.72)$

$\begin{array}{llr}\text { CH4 } & \text { G/MI } & .018 \\ \text { NMIC } & \text { G/MI } & .401 \\ \text { CARBONYL G/MI } & .813 \\ \text { ALCOHOL } & \text { G/MI } & 8.234\end{array}$

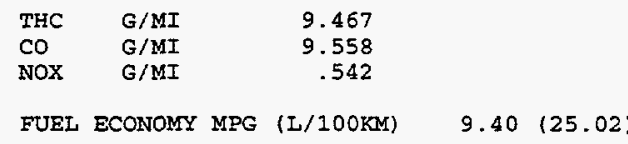


COMPUTER PROGRAM LDT $1.5-\mathrm{R}$

VEFICLE NUMBER 601

VEHICLE MODEL 88 CHEVY CORSICA

ENGTNE

2.8 L (171 CID) $-V-6$

2.8

15288 MILES ( $24598 \mathrm{KM})$

ODOMETER

IN HG $(743.7 \mathrm{MM} \mathrm{HG})$

BAROMETER 29.28 IN HG 1743.7

RELATIVE HUM

BAG DESCRIPTION

RUN TIME SECONDS

DRY/WET CORRECTION FACTOR, SAMP/BACK

MEASURED DISTANCE MILES (KM)

BLOWER FLOW RATE SCFM (SCMM)

GAS METER FLOW RATE SCFM (SCMM)

TOTAL FLOW SCF (SCM)

HC SAMPLE METER/RANGE/PPM (BAG)

HC BCKGRD METER/RANGE/PPM

CO SAMPLE METER/RANGE/PPM

CO BCKGRD METER/RANGE/PPM

$\mathrm{CO} 2$ SAMPLE METER/RANGE/PCT

CO2 BCKGRD METER/RANGE/PCT

NOX SAMPLE METER/RANGE/PPM (BAG) (D)

NOX BCKGRD METER/RANGE/PPM

CH4 SAMPLE PPM $(1.150)$

CH4 BCKGRD PPM

DILUTION FACTOR

HC CONCENTRATION PPM

CO CONCENTRATION PPM

$\mathrm{CO} 2$ CONCENTRATION PCT

NOX CONCENTRATION PPM

CH4 CONCENTRATION PPM

NMHC CONCENTRATION PPM

$\begin{array}{lll}\text { THC } & \text { MASS GRAMS } \\ \text { CO } & \text { MASS GRAMS } \\ \text { CO2 } & \text { MASS GRAMS } \\ \text { NOX } & \text { MASS GRAMS } \\ \text { CH4 } & \text { MASS GRAMS } \\ \text { NMHC } & \text { MASS GRAMS } & \text { (FID) } \\ \text { FUEL } & \text { MASS KG }\end{array}$

FUEI, MASS KG

FUEL ECONOMY MPG (I/100KM)

(BAG)
(BAG) (D)

EST RESEARCH INSTITUTE - DEPARTMENT OF EMIS
3-BAG CARB FTP VEHICLE EMISSION RESULTS

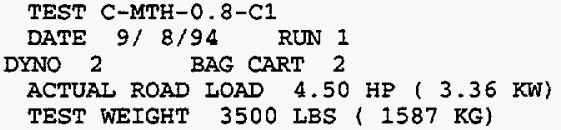

DRY BULB TEMPERATURE $72.0^{\circ} \mathrm{F}\left(22.2^{\circ} \mathrm{C}\right)$

$$
\begin{gathered}
1 \\
\text { COLD TRANSIENT } \\
(0-505 \text { SEC. }) \\
505.8 \\
.971 / .983 \\
3.63(5.84) \\
564.3(15.98) \\
.28\left(\begin{array}{c}
.01) \\
4759 .(134.8)
\end{array}\right.
\end{gathered}
$$

$48.7 / 2 / \quad 48.67$

8.01218 .00

$77.8 / 13 / 187.74$

.9/ $13 / 2.07$

$79.1 / 14 / .6428$

$\begin{array}{lll}79.1 / & 14 / & .6428 \\ 13.8 / & 14 / & .0470\end{array}$

$\begin{array}{rrr}13.8 / & 14 / & .0470 \\ 26.3 / & 1 / & 6.57\end{array}$

.41 1/ $\quad .10$

5.12

17.64

41.13

178.57

.5985

6.48

1.00

$-8.09$

11.247

28.020

1476.94

1.656

.090

.000

1.119

$9.73(24.18)$

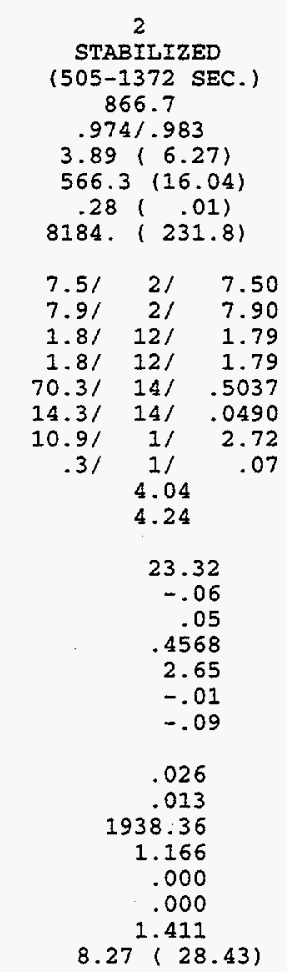

RESEARCH

PROJECT NO. 08-6068-001

METHANOL EM-1791-F

FUEL DENSITY $6.610 \mathrm{LB} / \mathrm{GAL}$

$\begin{array}{llllll}\mathrm{H} .123 & \mathrm{C} .375 & 0.502 & \mathrm{X} .000\end{array}$

NOX HUMIDITY C.F. .991

3
HOT TRANSTENT
$0-505$ SEC

$10-505$

$972 / .983$

$3.61(5.81)$

$563.5(15.96)$

$.28(.01)$

$4749 .(134.5)$

$71.7 / \quad 1 / 7.19$

$72.4 / \quad 1 / 7.26$

$12.4 / 12 / 12.19$

$2.0 / 12 / 1.99$

$75.5 / 14 / .5822$

$14.4 / \quad 14 / \quad .0494$

$28.8 / 1 / 7.20$

$.3 / \quad 1 / \quad .07$

4.15

4.13

20.15

.29

9.89

.5353

7.13

.22

025

1.549

1318.15

1. 817

.020

.003

.961

$11.27 ; 20.88\}$

3-BAG COMPOSITE RESULTS

$\begin{array}{llr}\text { THC } & \text { G/MI } & .648 \\ \text { CO } & \text { G/MI } & 1.721 \\ \text { NOX } & \text { G/MI } & .388\end{array}$

$\begin{array}{ll}\text { CH4 } & \text { G/MI } \\ \text { NMIC } & \text { G/MI } \\ \text { CARBONYL } & \text { G/MI } \\ \text { ALCOHOI } & \text { G/MI }\end{array}$

FUEL ECONOMY MPG (I/100KM)

$9.24(25.46)$

.007
.000
.022
.619 
COMPUTER PROGRAM LDT $1.5-\mathrm{R}$

VEHICLE NUMBER 601

VEHICLE MODEL 88 CHEVY CORSICA

ENGINE

2.8 L (17I CID) $-V-6$

M5

ODOMETER $\quad 15299$ MILES ( $24616 \mathrm{KM}$ )

BAROMETER 29.27 IN HG (743.5 MM HG) RELATIVE HUMIDITY 64.4 PCT.

BAG NUMBER

BAG DESCRIPTION

RUN TIME SECONDS

DRY/WET CORRECTION FACTOR, SAMP/BACK

MEASURED DISTANCE MILES (KM)

BLOWER FLOW RATE SCFM (SCMM)

GAS METER FLOW RATE SCFM (SCMM)

TOTAL FLOW SCF (SCM)

HC SAMPLE METER/RANGE/PPM (BAG)

HC BCKGRD METER/RANGE/PPM

CO SAMPLE METER/RANGE/PPM

CO BCKGRD METER/RANGE/PPM

$\mathrm{CO} 2$ SAMPLE METER/RANGE/PCT

CO2 BCKGRD METER/RANGE/PCT

NOX SAMPLE METER/RANGE/PPM

NOX BCKGRD METER/RANGE/PPM

CH4 SAMPIE PPM (1.150)

CH4 BCKGRD PQM

DILUTION FACTOR

HC CONCENTRATION PPM

CO CONCENTRATION PPM

$\mathrm{CO} 2$ CONCENTRATION PCT

NOX CONCENTRATION PPM

CH4 CONCENTPATION PPM

NMAC CONCENTRATION PPM

$\begin{array}{lll}\text { THC } & \text { MASS GRAMS } \\ \text { CO } & \text { MASS GRAMS } \\ \text { CO2 } & \text { MASS GRAMS } \\ \text { NOX } & \text { MASS GRAMS } \\ \text { CH4 } & \text { MASS GRAMS } \\ \text { NMHC } & \text { MASS GRAMS (FID) } \\ \text { FUEI } & \text { MASS KG } \\ \text { FUEI, ECONOMY MPG } & (\mathrm{L} / 100 \mathrm{KM})\end{array}$

FUEI, ECONOMY MPG (L/100KM $)$

3-BAG COMPOSITE RESULTS

$\begin{array}{llr}\text { THC } & \text { G/MI } & .661 \\ \text { CO } & \text { G/MI } & 1.994 \\ \text { NOX } & \text { G/MI } & .378\end{array}$

FUEL ECONOMY MPG (L/100KM)

$9.28(25.36)$
3-BAG CARB FTP VEHICIE EMISSION RESULTS

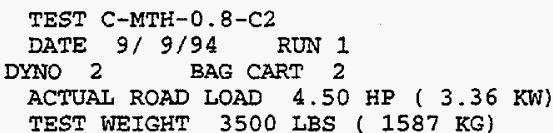

DRY BULE TEMPERATURE $71.0^{\circ} \mathrm{F}\left(21.7^{\circ} \mathrm{C}\right\}$

$$
1
$$

COLD TRANSIENT

$0-505$ SEC.)

505.2

$.970 / .983$

$3.59(5.78)$

$557.8(15.80)$

$.27(.01)$

4699 . (133.1)

$50.5 / 2 / 50.47$

6.81216 .80

$91.8 / 13 / 225.26$

$.9 / 13 / 2.07$

$79.1 / 14 / \quad .6428$

$\begin{array}{llll}13.0 / 14 / & .0438\end{array}$

$26.0 / 1 / 6.50$

$.21 \quad 11 \quad .05$

3.50

17.54

44.06

44.06
214.38

.6015

6.45

.94

$-5.61$

11.293

33.216

1465.70

1. 641

.083
.000

1. 116

$9.64(24.39)$

STABIZIZED

(505-1372 SEC.)

867.5

$.973 / .983$

$3.85(6.20)$

$559.1(15.83)$

$28(.01)$

8088 . (229.1)

$56.3 / 1 / \quad 5.65$

$58.5 / \quad 1 / \quad 5.87$

$1.0112 / \quad 99$

$1.0 \% 12 \%$

$69.91 \quad 141.4981$

$13.3 / 14 / .0450$

$10.6 / 21 / 2.65$

$.1 / 1 / \quad .02$

2.86

2.94

23.59

.03

.4550

2.63

.05

.044

.007

1908.10

1.149

.007

.000

1.389

$8.31(28.30)$

CH4

MNHC

G/MI

M/MI

ALCOHOL G/MI
RESEARCH

PROJECT NO. 08-6068-001

METHANOL EM-179I-F

FUEL DENSITY $6.610 \mathrm{LB} / \mathrm{GAI}$

$\begin{array}{lllllll}\mathrm{F} .123 & \mathrm{C} & .375 & 0 & .502 & \mathrm{X} .000\end{array}$

NOX HUMIDITY C.F. .999

HOT TRANSIENT

( $0-505$ SEC.)

506.0

$.972 / .983$

$3.60(5.79)$

$560.8(15.88)$

$28(.01)$

4732 . (134.0)

$55.6 / \quad 1 / 5.58$

$54.6 / 1 / 5.48$

$7.4 / 12 / 7.31$

$.91 \quad 121 \quad .90$

$\begin{array}{llll}74.9 / & 14 / & .5727\end{array}$

$13.0 / 14 / \quad .0438$

$26.4 / \quad 1 / 6.60$

$.1 / \quad 1 / \quad .02$

2.75

2.62

20.50

.37

6.20

.5310

6.57

.26

.030

.967

1302.79

1.684

023

005

.950

$11.37(20.69)$ 
COMPUTER PROGRAM LDT $1.5-R$

SOUTEWEST RESEARCH INSTITUTE - DEPARTMENT OF EMISSIONS RESEARCH
PROJECT NO. 08-6068-001

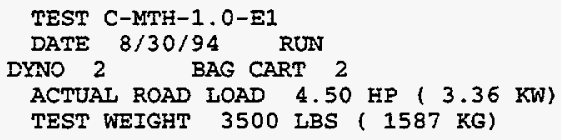

DRY BULB TEMPERATURE $76.0^{\circ} \mathrm{F}\left(24.4^{\circ} \mathrm{C}\right)$

BAROMETER 29.20 IN HG $(741.7 \mathrm{MM}$ HG) RELATIVE HUMIDITY 52.0 PCT

BAG NUMBER

BAG DESCRIPTION

RUN TIME SECONDS

DRY/WET CORRECTION FACTOR, SAMP/BACK

MEASURED DISTANCE MILES (KM)

BLOWER FLOW RATE SCFM (SCMM)

GAS METER FLOW RATE SCFM (SCMM)

TOTAL FLOW SCF (SCM)

\begin{tabular}{|c|c|c|}
\hline HC & SAMPLE METER/RANGE/PPM & (BAG) \\
\hline $\mathrm{HC}$ & BCKGRD METER/RANGE/PPM & \\
\hline $\mathrm{CO}$ & SAMPLE METER/RANGE/PPM & \\
\hline co & BCKGRD METER/RANGE/PPM & \\
\hline $\mathrm{CO} 2$ & SAMPLE METER/RANGE/PCT & \\
\hline $\mathrm{CO} 2$ & BCRGRD METER/RANGE/PCT & \\
\hline NoX & SAMPLE METER/RANGE/PPM & (BAG) (D) \\
\hline Nox & BCKGRD METER/RANGE/PPM & \\
\hline $\mathrm{CF} 4$ & SAMPLE PPM (1.150) & \\
\hline CH4 & BCKGRD PPM & \\
\hline \multicolumn{3}{|c|}{ DILUTION FACTOR } \\
\hline $\mathrm{HC}$ & CONCENTRATION PPM & \\
\hline $\mathrm{CO}$ & CONCENTRATION PPM & \\
\hline $\mathrm{CO} 2$ & CONCENTRATION PCT & \\
\hline NOX & CONCENTRATION PPM & \\
\hline $\mathrm{CH} 4$ & CONCENTRATION PPM & \\
\hline NMEC & C CONCENTRATION PPM & \\
\hline THC & MASS GRAMS & \\
\hline $\mathrm{CO}$ & MASS GRAMS & \\
\hline $\mathrm{CO} 2$ & MASS GRAMS & \\
\hline NOX & MASS GRAMS & \\
\hline CH4 4 & MASS GRAMS & \\
\hline NMHC & MASS GRAMS (FID) & \\
\hline VUEL & MASS KG & \\
\hline
\end{tabular}

$$
\begin{gathered}
1 \\
\text { COLD TRANSIENT } \\
(0-505 \text { SEC. }) \\
505.3 \\
.972 / .984 \\
3.62(5.83) \\
555.2(15.72) \\
.26(.01) \\
4678 .(132.5)
\end{gathered}
$$$$
\begin{array}{rrr}
12.0 / & 3 / & 119.73 \\
.9 / & 3 / & 8.98 \\
96.0 / & 14 / & 480.25 \\
.6 / & 14 / & 2.43 \\
77.4 / & 14 / & .6135 \\
15.2 / & 14 / & .0527 \\
42.8 / & 1 / & 10.70 \\
.3 / & 1 / & .07 \\
& 5.49 & \\
& 3.71
\end{array}
$$$$
17.43
$$$$
111.27
$$$$
461.24
$$$$
\begin{array}{r}
461.24 \\
.5638
\end{array}
$$$$
\begin{array}{r}
.5638 \\
10.63
\end{array}
$$$$
1.99
$$$$
-27.60
$$

32.507

71.142

1367.68

2.649

.176

1.110

$9.78(24.04)$
METHANOL EM-1791-F

FUEI DENSITY 6.610 IBB/GAI

F. $.123 \quad \mathrm{C} .3750 .502 \quad \mathrm{X} .000$

NOX HUMIDITY C.F. .984

$$
\begin{aligned}
& 3 \\
& 505.2 \\
& 973 / 984
\end{aligned}
$$

$\begin{array}{rrr}57.6 / & 2 / & 57.57 \\ 7.9 / & 2 / & 7.90 \\ 93.7 / & 13 / & 230.35 \\ .7 / & 13 / & 1.61 \\ 66.0 / & 14 / & .4457 \\ 14.4 / & 14 / & .0494 \\ 15.6 / & 1 / & 3.90 \\ .2 / & 1 / & .05 \\ & 4.47 & \\ & 3.82 & \end{array}$

$$
\begin{array}{r}
24.78 \\
49.99 \\
221.93 \\
.3983 \\
3.85 \\
.81 \\
5.48
\end{array}
$$

19.092

58.972

1664.37

1.654

.123

.721

$85^{1.298}\{26.59\}$

20.64
70.63
261.82
.4853
11.55
.86
3.00

16.274

40.584

1182.94

2.894

.076

.230

.924

$11.74 \div(20.04)$
3-BAG COMPOSITE RESULTS

$\begin{array}{llr}\text { THC } & \text { G/MI } & 5.684 \\ \text { CO } & \text { G/MI } & 15.127 \\ \text { NOX } & \text { G/MI } & .596\end{array}$

CH4

NMFiC

G/MI

CARBONYL G/MI

ALCOHOL G/MI
.032
.114
.442
.095
FUEL ECONOMY MPG (L/100KM) 9.72 (24.21) 
COMPUTER PROGRAM LDT $1.5-\mathrm{R}$

$\begin{array}{lcl}\text { VEHICLE NUMBER } & 601 \\ \text { VEHICLE MODEL } & 88 \text { CHEVY CORSICA } \\ \text { ENGINE } & 2.8 \text { I (171 CID) -V -6 } \\ \text { TRANSMISSION } & \text { M5 } \\ \text { ODOMETER } & 15184 \text { MILES ( } 24431 \mathrm{KM})\end{array}$

BAROMETER 29.22 IN HG (742.2 MM HG) REIATIVE HUMIDITY 60.9 PCT.

BAG NUMBER

BAG DESCRIPTION

RUN TIME SECONDS

DRY/WET CORRECTION FACTOR, SAMP/BACK

MEASURED DISTANCE MILES (KM)

BLOWER FLOW RATE SCFM (SCMM)

GAS METER FLOW RATE SCFM (SCMM)

TOTAL FLOW SCF (SCM)

HC SAMPLE METER/RANGE/PPM (BAG)

HC BCKGRD METER/RANGE/PPM

CO SAMPLE METER/RANGE/PPM

CO BCKGRD METER/RANGE/ PPM

CO2 SAMPLE METER/RANGE/PCT

CO2 BCKGRD METER/RANGE/PCT

NOX SAMPLE METER/RANGE/PPM (BAG) (D)

NOX BCKGRD METER/RANGE/PPM

CH4 SAMPLE PPM $(1.150)$

CH4 BCKGRD PPM

\section{DILUTION FACTOR}

HC CONCENTRATION PPM

CO CONCENTRATION PPM

$\mathrm{CO} 2$ CONCENTRATION PCT

NOX CONCENTRATION PPM

CE4 CONCENTRATION PPM

NMHC CONCENTRATION PPM

$\begin{array}{lll}\text { THC } & \text { MASS GRAMS } \\ \text { CO } & \text { MASS GRAMS } & \\ \text { CO2 } & \text { MASS GRAMS } & \\ \text { NOX } & \text { MASS GRAMS } & \\ \text { CH4 } & \text { MASS GRAMS } & \\ \text { MMHC } & \text { MASS GRAMS } & \text { (FID) } \\ \text { FUEL } & \text { MASS KG } & \end{array}$

FUEL ECONOMY MPG (L/100KM)

3-BAG COMPOSITE RESULTS

SOUTHWEST RESEARCH INSTITUTE - DEPARTMENT OF EMISSIONS RESEARCH

3-BAG CARB FTP VEHICLE EMISSION RESULTS

PROJECT NO. 08-6068-001

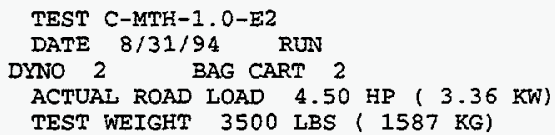

DRY BULB TEMPERATURE $72.0^{\circ} \mathrm{F}\left(22.2^{\circ} \mathrm{C}\right)$

$$
1
$$

$$
\begin{aligned}
& \text { COLD TRANSIENT } \\
& (0-505 \text { SEC. }) \\
& 505.1 \\
& .972) .983 \\
& 3.62(5.82) \\
& 557.0(15.77) \\
& .27\left(\begin{array}{c}
1 \\
4691 .(132.9)
\end{array}\right.
\end{aligned}
$$

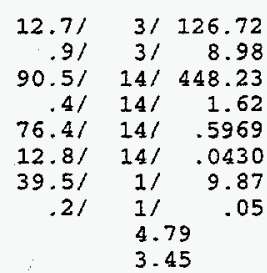

37.94

17.94
138.24

118.24
430.01

.5562

9.82

1.53

29.921

66.511

1353.06

2.477

.136

1. 091

$9.94(23.67)$
STABILIZED

(505-1372 SEC.)

867.5

$.975 / .983$

$3.88(6.24)$

$558.8(15.83)$

$28(.01)$

8083 . (228.9)

$\begin{array}{rrr}55.4 / & 2 / & 55.37 \\ 8.1 / & 2 / & 8.10 \\ 46.2 / & 14 / & 203.02 \\ .3 / & 14 / & 1.21 \\ 65.3 / & 14 / & .4368 \\ 13.1 / & 14 / & .0442 \\ 16.0 / & 1 / & 4.00 \\ .2 / & 1 / & .05 \\ & 4.01 & \\ & 3.46 & \end{array}$

25.42

25.42
47.59

47.59
195.26

.3943

3.95

.68

18.329

52.038

1652.73

1.716

.104

.562

1. 281

$9.07(25.93)$
METHANOL EM-1791-F

FUEL DENSITY 6.610 LB/GAL

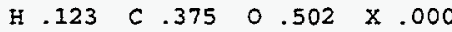

NOX HUMTDITY C.F. $\quad .992$

$$
3
$$

HOT TRANSIENT

( $0-505$ SEC.)

505.1

$.973 / .983$

$3.58(5.76)$

$559.5(15.84)$

$.27(.01)$

4712. (133.4)

$72.4 / 2 / \quad 72.36$

7.41217 .40

$52.9 / 14 / 236.93$

$.3 / 14 / 1.21$

$71.8 / \quad 14 / \quad .5254$

$13.8 / 14 / .0470$

$37.7 / \quad 1 / 9.42$

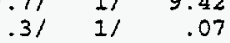

4.06
3.38

$$
\begin{array}{r}
21.13 \\
65.31 \\
227.45 \\
.4806 \\
9.35 \\
.84 \\
43
\end{array}
$$

15.443

35.336

1174.18

2. 368

.075

.033

$11.79(19.95)$

$\begin{array}{llr}\text { THC } & \text { G/MI } & 5.351 \\ \text { CO } & \text { G/MI } & 13.482 \\ \text { NOX } & \text { G/MI } & .553\end{array}$

FUEL ECONOMY MPG (L/100KM)
$9.89(23.79)$ $\begin{array}{llr}\text { CH4 } & \text { G/MI } & .027 \\ \text { NMHC } & \text { G/MI } & .078 \\ \text { CARBONYI } & \text { G/MI } & .397 \\ \text { ALCOHOL } & \text { G/MI } & 4.848\end{array}$ 
COMPUTER PROGRAM LDT $1.5-\mathrm{R}$

VEHICLE NUMBER VEHICLE MODEL ENGINE TRANSMISSION 88 CHEVY CORSICA. 2.8 I (17I CID) $-V-6$ M5

ODOMETER

15152 MILES ( $24379 \mathrm{KM})$

BAROMETER 29.23 IN HG (742.4 MM HG) RELATIVE HUMIDITY 60.4 PCT.

BAG NUMBER

BAG DESCRIPTION

RUN TIME SECONDS

DRY/WET CORRECTION FACTOR, SAMP/BACK

MEASURED DISTANCE MILES (KM)

BLOWER FLOW RATE SCFM (SCMM)

GAS METER FLOW RATE SCFM (SCMM)

TOTAL FLOW SCF (SCM)

HC SAMPLE METER/RANGE/PPM (BAG)

HC BCRGRD METER/RANGE/PPM

CO SAMPLE METER/RANGE/PPM

CO BCKGRD METER/RANGE/PPM

CO2 SAMPLE METER/RANGE/PCT

CO2 BCKGRD METER/RANGE/PCT

NOX SAMPLE METER/RANGE/PPM (BAG) (D)

NOX BCKGRD METER/RANGE/PPM

CH4 SAMPIE PPM (1.150)

CH4 BCKGRD PPM

DILUTION FACTOR

HC CONCENTRATION PPM

CO CONCENTRATION PPM

$\mathrm{CO} 2$ CONCENTRATION PCT

NOX CONCENTRATION PPM

CH4 CONCENTRATION PPM

NMHC CONCENTRATION PPM

$\begin{array}{ll}\text { THC } & \text { MASS GRAMS } \\ \text { CO } & \text { MASS GRAMS } \\ \text { CO2 } & \text { MASS GRAMS } \\ \text { NOX } & \text { MASS GRAMS } \\ \text { CH4 } & \text { MASS GRAMS } \\ \text { NMHC } & \text { MASS GRAMS (FID) } \\ \text { FUEL } & \text { MASS KG }\end{array}$

FUEL ECONOMY MPG $(\mathrm{I} / 100 \mathrm{KM})$

3-BAG COMPOSITE RESULTS

$\begin{array}{llr}\text { THC } & \text { G/MI } & .518 \\ \text { CO } & \text { G/MI } & 2.143 \\ \text { NOX } & \text { G/MI } & .348\end{array}$

FUEI ECONOMY MPG (L/100KM)

$9.68(24.30)$

$$
\begin{aligned}
& \text { COLD TRANSIENT } \\
& (0-505 \text { SEC. }) \\
& 505.1 \\
& .972(.984 \\
& 3.58(5.76) \\
& 557.2(15.78) \\
& .27\left(\begin{array}{c}
.01) \\
4693 .(132.9)
\end{array}\right.
\end{aligned}
$$

$47.5 / 2 / \quad 47.47$

$9.2 / 2 / \quad 9.19$

$50.7 / 14 / 225.64$

$.2 / 14 / \quad .81$

$78.3 / \quad 14 / \quad .6289$

$13.0 / 14 / \quad .0438$

$29.1 / 1 / 7.27$

$\begin{array}{lll}.31 & 1 / & .07\end{array}$

5.88

4.97

$$
17.93
$$

38.79

216.30

.5875

7.20

1.19

62

8.701
33.467

1429.63

1.790

.105

.047

1.088

$9.86(23.85)$

.518

.348

(I/100KM)

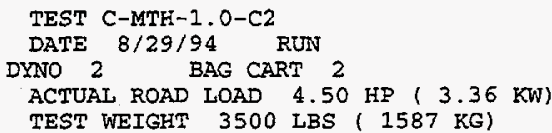

DRY BULB TEMPERATURE $71.0^{\circ} \mathrm{F}\left(21.7^{\circ} \mathrm{C}\right)$

STABIIIZED

(505-1372 SEC.)

867.5

$.975 / .984$

$3.82(6.15)$

$558.9(15.83)$

$28(.01)$

8084 . (229.0)

$8.8 / 21 \quad 8.79$

$8.8 / 2 / \quad 8.79$

$1.6 / 12 / 1.63$

$.8 / 12 / \quad .82$

$67.8 / 14 / .4693$

$13.6 / 14 / \quad .0462$

$8.8 / 1 / 2.20$

$.2 / 1 / \quad .05$

4.89

4.83

25.02

.35

.81
.4249

2.15

.26

.05

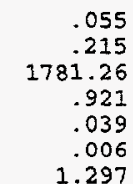

$8.83(26.63)$

CH. 4

NMIHC

G/MI

CARBOTr G/MI

ALCOHOL G/MI
RESEARCH

PROJECT NO. 08-6068-001

METHANOL EM-1791-F

FUEI DENSITY $6.610 \mathrm{IB} / \mathrm{GAI}$

H $.123 \quad \mathrm{C} .3750 .502 \quad \mathrm{X} .000$

NOX HUMIDITY C.F. $\quad .978$

3

HOT TRANSIENT

( $0-505$ SEC.)

505.1

$.973 / .984$

$3.60(5.80)$

$559.5(15.85)$

$.27(.01)$

4712 . ( 133.5$)$

$\begin{array}{lll}7.2 / & 21 & 7.20\end{array}$

$\begin{array}{lll}7.0 / 21 & 21 & 7.00\end{array}$

$15.7 / \quad 12 / 35.68$

$1.0 / 12 / 1.02$

$74.7 / 14 / \quad .5695$

$13.7 / 14 / \quad .0466$

$25.3 / 1 /$

$\begin{array}{lll}.31 & 11 & .07\end{array}$

4.55
4.20

$$
\begin{array}{r}
20.58 \\
.54 \\
14.16 \\
.5252 \\
6.25 \\
.56 \\
-.15
\end{array}
$$

.065

2.200

1283.21

1.561

.050

.000

$11.53(20.41)$ 
SOUTHWEST RESEARCH INSTITUTE - DEPARTMENT OF EMISSIONS RESEARCH

COMPUTER PROGRAM LDT $1.2-\mathrm{R}$ 3-BAG CARB FTP VEHICLE EMISSION RESULTS

PROUECT NO. 08-6068-001

VEFICLE NUMBER 601

VEHICLE MODEL 88 CHEVY CORSICA

ENGINE

TRANSMISSION

2.8 I ( 171 CID) $-\mathrm{V}-6$

M5

15377 MILES ( $24741 \mathrm{KM})$

BAROMETER 29.20 IN HG $(741.7 \mathrm{MM} \mathrm{HG})$ RELATIVE HUMIDITY 60.9 PCT.

BAG NUMBER

BAG DESCRIPTION

RUN TIME SECONDS

DRY/WET CORRECTION FACTOR, SAMP/BACK

MEASURED DISTANCE MILES (KM)

BLOWER FLOW RATE SCFM (SCMM)

GAS METER FLOW RATE SCFM (SCMM)

TOTAI FLOW SCF (SCM)

HC SAMPLE METER/RANGE/PPM (BAG)
HC BCKGRD METER/RANGE/PPM
CO SAMPLE METER/RANGE/PPM
CO BCKGRD METER/RANGE/PPM
CO2 SAMPLE METER/RANGE/PCT
CO2
BCKGRD METER/RANGE/PCT
NOX SAMPLE METER/RANGE/PPM (BAG) (D)
NOX BCKGRD METER/RANGE/PPM
CH4 SAMPLE PPM (1.15O)
CH4 BCKGRD PPM
DILUTION FACTOR
HC CONCENTRATION PPM
CO CONCENTRATION PPM
CO2 CONCENTRATION PCT
NOX CONCENTRATION PPM
CH4 CONCENTRATION PPM
NMHC CONCENTRATION PPM

CONCENTRATION PRM

$\begin{array}{ll}\text { THC } & \text { MASS GRAMS } \\ \text { CO } & \text { MASS GRAMS } \\ \text { CO2 } & \text { MASS GRAMS } \\ \text { NOX } & \text { MASS GRAMS } \\ \text { CH4 } & \text { MASS GRAMS } \\ \text { NMHC } & \text { MASS GRAMS (FID) } \\ \text { FUEL } & \text { MASS KG }\end{array}$

FUEL ECONOMY MPG (L/10OKM)

$$
\begin{aligned}
& \text { TEST C-MTH-1.2-EI } \\
& \text { DATE } 9 / 16 / 94 \quad \text { RUN } \\
& \text { DYNO } 2 \text { BAG CART } 2 \\
& \text { ACTUAL ROAD LOAD } 4.50 \mathrm{HP}(3.36 \mathrm{~kW}) \\
& \text { TEST WEIGHT } 3500 \text { LBS ( } 1587 \mathrm{KG})
\end{aligned}
$$

DRY BULB TEMPERATURE $72.0^{\circ} \mathrm{F}\left(22.2^{\circ} \mathrm{C}\right)$

$$
\begin{gathered}
\text { I } \\
\text { COLD TRANSIENT } \\
(0-505 \text { SEC. }) \\
506.4 \\
.971 / .983 \\
3.61(5.81) \\
557.6(15.79) \\
.28(-.01) \\
4708 .(133.3)
\end{gathered}
$$

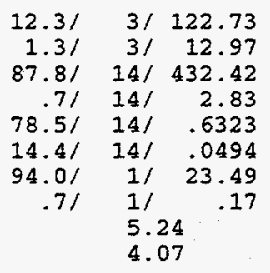

17.04

110.52

413.22

.5858

23.33

1.42

$-61.91$

40.084

64.141

1430.08

5.904

.126

.000

1.155

$9.37(25.09)$

3-BAG COMPOSITE RESULTS

$\begin{array}{llr}\text { THC } & \text { G/MI } & 5.061 \\ \text { CO } & \text { G/MI } & 18.273 \\ \text { NOX } & \text { G/MI } & 1.507\end{array}$

FUEI ECONOMY MPG (L/100KM)
$\mathrm{CH} 4$

NMLHC

G/MI

CARBONYL $G / M I$
AICOHOL G/MI

\section{2}

STABILIZED (505-1372 SEC.)

$$
867.3
$$$$
974 / .983
$$

$3.84(6.17)$

$557.1(15.78)$

$.28(.01)$

$8056 .(228.2)$

$39.7 / 2 / \quad 39.68$

$6.0121 \quad 6.00$

$66.6 / 14 / 310.43$

$.7 / 14 / 2.83$

$66.6 / 14 / .4535$

$12.0 / 14 / .0399$

$46.8 / 1 / 11.70$

$.01 \quad 1 / \quad .00$

3.02

24.10

33.93

297.50

.4152

11.70

.83
-1.00

14.110

14.110
79.020

79.020
1734.38

5.066

.126

.000

1.367

$8.41(27.97)$

METHANOL EM-1791-F

FUEI DENISITY $6.610 \mathrm{LB} / \mathrm{GAI}$

H .123 C $.3750 .502 \quad \mathrm{X} .000$

NOX HUMIDITY C.F. .993

3

HOT TRANSIENT

( $0-505$ SEC.)

505.8

9721.983

$3.62(5.82)$

$556.9(15.77)$

$27(.01)$

4697. (133.0)

$4.5 / 3 / 44.90$

$.8 / 3 / 7.98$

$73.6 / 14 / 349.89$

$.6 / 14 / 2.43$

$74.0 / 14 / .5586$

$14.1 / 14 / \quad .0482$

$25.4 / 2 / 25.41$

$.21 \quad 21 \quad .20$

4.43

3.48

19. 68

37.32

334.98

.5128

25.22

1.13

$-9.58$

11.160

51.870

1248.79

6.368

.100

.000

$11.08(21.23)$
.032

.000

4.671 
COMPUTER PROGRAM LDT $1.5-\mathrm{R}$

SOUTHWEST RESEARCH INSTITUTE - DEPARTMENT OF EMISSIONS RESEARCH

3-BAG CARE FTP VEHICLE EMISSION RESULTS

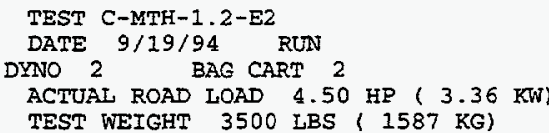

DRY BULB TEMPERATURE $75.0^{\circ} \mathrm{F}\left(23.9^{\circ} \mathrm{C}\right)$$$
1
$$

$$
\begin{aligned}
& \text { COLD TRANSIENT } \\
& (0-505 \text { SEC. }) \\
& 505.3 \\
& .975 / .987 \\
& 3.61(5.80) \\
& 558.4(15.81) \\
& .28(1.01) \\
& 4705 .(133.3)
\end{aligned}
$$

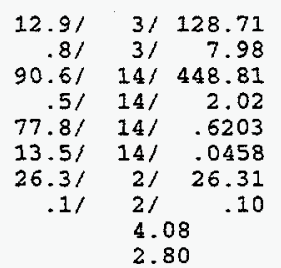

17.30

17.30
121.19

432.29

.5772

26.22

1.44

$-19.45$

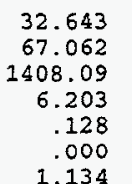

$9.53(24.69)$
2

STABILIZED

(505-1372 SEC.)

867.2

$.978 / .987$

$3.86(6.21)$

$560.4(15.87)$

$.28(.01)$

$8103 .\langle 229.5\}$

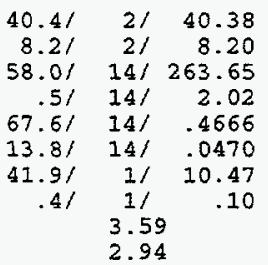

23.67
32.53

254.34

.4216

10.38

.78

$-2.25$

$$
\begin{array}{r}
14.358 \\
67.951 \\
1771.48 \\
4.228 \\
.120 \\
.000 \\
1.382
\end{array}
$$

$8.38(28.08)$
PROJECT NO. 08-6058-001

METHANOL EM-1791-F

FUEL DENSITY $6.610 \mathrm{LB} / \mathrm{GAI}$

H $.123 \quad \mathrm{C} .375 \quad 0.502 \quad \mathrm{X} .000$

NOX HUMIDITY C.F. .928

HOT TRANSIENT

( $0-505$ SEC.)

505.8

$.976 \% .987$

$3.61(5.81)$

$560.2(15.87)$

$.28(.01)$

$4725 .(133.8\}$

$\begin{array}{lll}41.3 / & 2 / & 41.28\end{array}$

$75 / 21750$

$69.51 \quad 141327.20$

$\begin{array}{rrr}69.5 / & 14 / & 327.20 \\ .5 / & 14 / & 2.02\end{array}$

$\begin{array}{lll}74.6 / & 14 / & .5679\end{array}$

$14.1 / 14 / \quad 0482$

$87.7 / 3 / 21 / 92$

$\begin{array}{rrr}.5 / 11 & 11 & .12\end{array}$

3.98

3.15

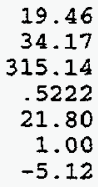

9.476

49.091

1279.29

5.179

.089
.000

.997

$10.86(21.67)$

3-BAG COMPOSITE RESULTS

$\begin{array}{llr}\text { THC } & \text { G/MI } & 4.526 \\ \text { NO } & \text { G/MI } & 16.708 \\ \text { NOX } & \text { G/MI } & 1.319\end{array}$

FUEI ECONOMY MPG
16.708
1.319

(I/100KM)
cHe

NMHC

G/MI

CARBONTT G/MI

ALCOHOL G/MI
.030

1000

.336
4.160 
COMPUTER PROGRAM LDT $1.5-\mathrm{R}$

VEHICLE NUMBER 601

VEHICLE MODEL 88 CHEVY CORSICA

ENGINE

TRANSMISSION

2.8 I (171 CID) $-\mathrm{V}-6$

M5

15348 MILES ( $24694 \mathrm{KM}$ )

BAROMETER 29.28 IN HG $(743.7 \mathrm{MM} \mathrm{HG})$

RELATIVE HUMIDITY 64.9 PCT.

BAG NUMBER

BAG DESCRIPTION

RUN TIME SECONDS

DRY/WET CORRECTION FACTOR, SAMP/BACK

MEASURED DISTANCE MILES (KM)

BLOWER FLOW RATE SCFM (SCMM)

GAS METER FLOW RATE SCFM (SCMM)

TOTAL FLOW SCF (SCM)

HC SAMPLE METER/RANGE/PPM (BAG)

HC BCKGRD METER/RANGE/PPM

CO SAMPLE METER/RANGE/PPM

CO BCKGRD METER/RANGE/PPM

CO2 SAMPLE METER/RANGE/PCT

$\mathrm{CO} 2$ BCKGRD METER/RANGE/PCT

NOX SAMPLE METER/RANGE/PPM (BAG) (D)

NOX BCKGRD METER/RANGE/PPM

CH4 SAMPLE PPM $(1.150)$

CH4 BCKGRD PPM

DILUTION FACTOR

HC CONCENTRATION PQM

CO CONCENTRATION PPM

$\mathrm{CO} 2$ CONCENTRATION PCT

NOX CONCENTRATION PPM

CH4 CONCENTRATION PPM

NMHC CONCENTRATION PPM

THC MASS GRAMS

CO MASS GRAMS

$\mathrm{CO} 2$ MASS GRAMS

NOX MASS GRAMS

CH4 MASS GRAMS

NMHC MASS GRAMS (FID)

FUEI MASS KG

FUEI ECONOMY MPG (I/100KM)
TEST C-MTH-1.2-C2
DATE $9 / 14 / 94 \quad$ RUN

DYNO 2 BAG CART 2

ACTUAL ROAD LOAD $4.50 \mathrm{HP}(3.36 \mathrm{KW})$

TEST WEIGHT 3500 I.BS ( $1587 \mathrm{KG}$ )

DRY BULB TEMPERATURE $72.0^{\circ} \mathrm{F}\left(22.2^{\circ} \mathrm{C}\right)$

$$
\begin{gathered}
1 \\
\text { COLD TRANSIENT } \\
(0-505 \text { SEC. }) \\
505.3 \\
.969 / .982 \\
3.57(5.74) \\
558.1(15.81) \\
.28(0.01) \\
4702 .(133.2)
\end{gathered}
$$

$\begin{array}{lll}69.91 & 21 & 69.86\end{array}$

$8.0121 \quad 8.00$

$29.6 / 14 / 124.70$

$.4 / 14 / 1.62$

$81.7 / 14 / .6903$

$13.7 / 14 / \quad .0466$

$\begin{array}{lll}7.4 / & 21 & 7.40 \\ .01 & 21 & 00\end{array}$

3.8

3.21

16.55

62.35

118.06

.6465

7.40

.81
-6.72

15.665

18.303

1576.26

1.913

.072

.000

1.184

$9.04(26.03)$

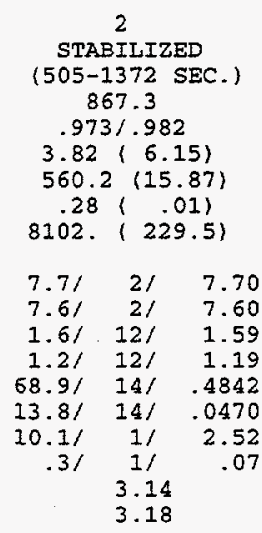

24.26

.41

.4391

.4391
2.45

.09
.29

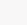

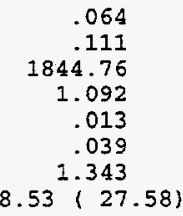

$8.53(27.58)$

CH4 G/MI

NMLHC G/MI

CARBONYL G/MI

ALCOHOL G/MI
RESEARCH

PROJECT NO. 08-6068-001

METHANOL EM-1791-F

FUEL DENSITY $6.610 \mathrm{LB} / \mathrm{GAL}$

H .123 C . $375 \quad 0.502 \quad \times \quad .000$

NOX HUMIDITY C.F. 1.014

HOT TRANSIENT

( $0-505$ SEC.)

505.4

$971 / .982$

$3.59(5.77)$

$557.5(15.79)$

$.27(.01)$

4698. (133.1)

$9.8 / 21 \quad 9.79$

$\begin{array}{lll}7.1 / 2 / 27 & 7.10\end{array}$

$59.9 / 13 / 141.02$

$.7 / 13 / 1.61$

$75.9 / \quad 14 / \quad .5887$

$14.5 / 14 / .0498$

$73.7 / \quad 1 / 18.42$

$.31 \quad 1 / \quad .07$

4.55

3.30

19.51

3.06

134.11

.5414

18.35

1.42
-1.69

.820

20.774

1318.92

4.736

.126

.000

$10.92 \div(21.53)$

3-BAG COMPOSITE RESULTS

$\begin{array}{llr}\text { THC } & \text { G/MI } & .983 \\ \text { CO } & \text { G/MI } & 2.678 \\ \text { NOX } & \text { G/MI } & .623\end{array}$

FUEI ECONOMY MPG (L/100KM)

$9.21(25.54)$ 
COMPUTER PROGRAM IDT $1.5-\mathrm{R}$

$\begin{array}{lc}\text { VEHICLE NUMBER } & 601 \\ \text { VEHICLE MODEL } & 88 \text { CHEVY CORSICA } \\ \text { ENGINE } & 2.8 \text { L (171 CID) }-V-6 \\ \text { TRANSMISSION } & \text { M5 } \\ \text { ODOMETER } & 15359 \text { MILES ( } 24712 \mathrm{KM})\end{array}$

BAROMETER 29.20 IN HG (741.7 MM HG) RELATIVE HUMIDITY 68.2 PCT.

BAG NUMBER

BAG DESCRIPTION

RUN TIME SECONDS

DRY/WET CORRECTION FACTOR, SAMP/BACK

MEASURED DISTANCE MIIES (KM)

BLOWER FLOW RATE SCFM (SCMM)

GAS METER FLOW RATE SCFM (SCMM)

TOTAL FLOW SCF (SCM)

HC SAMPLE METER/RANGE/PPM (BAG)

HC BCKGRD METER/RANGE/PPM

CO SAMPLE METER/RANGE/PPM

CO BCKGRD METER/RANGE/PPM

CO2 SAMPLE METER/RANGE/PCT

CO2 BCKGRD METER/RANGE/PCT

NOX SAMPLE METER/RANGE/PPM (BAG) (D)

NOX BCKGRD METER/RANGE/PPM

CH4 SAMPLE PPM $(1.150)$

CH4 BCKGRD PPM

DILUTION FACTOR

HC CONCENTRATION PPM

CO CONCENTRATION PPM

$\mathrm{CO} 2$ CONCENTRATION PCT

NOX CONCENTRATION PPM

CH4 CONCENTRATION PPM

NMHC CONCENTRATION PPM

THC MASS GRPMS

CO MASS GRAMS

$\mathrm{CO} 2$ MASS GRAMS

NOX MASS GRAMS

CH4 MASS GRAMS

NMHC MASS GRAMS (FID)

FUEL MASS KG

FUEL ECONOMY MPG (L/10OKM)

3-BAG COMPOSITE RESULTS

$\begin{array}{lll}\text { THC } & \text { G/MI } & .795 \\ \text { CO } & \text { G/MI } & 4.027 \\ \text { NOX } & \text { G/MI } & 1.032\end{array}$

FUEL ECONOMY MPG (L/100KM)

$8.97(26.22)$

THC

$$
\text { NOX }
$$

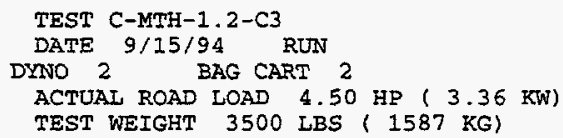

DRY BULB TEMPERATURE $70.0^{\circ} \mathrm{F}\left(21.1^{\circ} \mathrm{C}\right)$

$$
\begin{gathered}
1 \\
\text { COLD TRANSIENT } \\
(0-505 \text { SEC. }) \\
505.2 \\
.970 / .983 \\
3.60(5.78) \\
556.3(15.76) \\
.28(c .01) \\
4687 .(132.7)
\end{gathered}
$$

$\begin{array}{rrr}57.8 / & 2 / & 57.77 \\ 8.3 / & 2 / & 8.30 \\ 46.8 / & 14 / & 206.00 \\ .4 / & 14 / & 1.62 \\ 80.5 / & 14 / & .6680 \\ 13.8 / & 14 / & .0470 \\ 73.6 / & 1 / & 18.39 \\ .5 / & 1 / & .12 \\ & 4.73 & \\ & 3.63\end{array}$

16.94

49.96

195.91

.6238

18.28

1.32

$-2.57$

11.856

30.271

1515.72

4.678

.117

.000
1.150

$9.37(25.09)$
2

STABIIIZED

(505-1372 SEC.)

867.3

$.973 / .983$

$3.83(6.16\}$

$558.4(15.82)$

$.28(.01)$

$8076 .(228.7)$

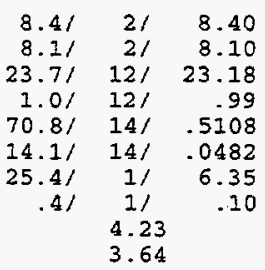

22.90

.65

21.39

.4648

6.25

.75
-.21

.122

5.694

2.758

2.758
.115
.000

.115
.000

1.423

$8.06(29.18)$

$\begin{array}{lll}\text { CH4 } & \text { G/MI } & .032 \\ \text { NMHC } & \text { G/MI } & .000 \\ \text { CARBONYL } & \text { G/MI } & .028 \\ \text { ALCOHOI } & \text { G/MI } & .735\end{array}$
PROJECT NO. 08-6068-001

METHANOL EM-1791-F

FUEL DENSITY $6.610 \mathrm{LB} / \mathrm{GAI}$

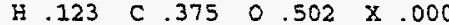

NOX HUMIDITY C.F. 1.008

HOT TRANSIENT

( $0-505$ SEC.)

505.2

$.971 / .983$

$3.55(5.71)$

$557.3(15.78)$

$.27(.01)$

4695. ( 133.0$)$

$11.5 / 2 / \quad 11.49$

$\begin{array}{lll}7.2 / 21 & 7.20\end{array}$

$56.7 / 13 / 132.91$

$.9 / 13 / 2.07$

$75.7 / 14 / .5855$

$14.6 / 14 / \quad .0502$

$78.8 / 1 / 19.69$

.5) $1 / \quad .12$

4.91

3.69

$$
\begin{array}{r}
19.63 \\
4.66 \\
125.76 \\
.5378 \\
19.58 \\
1.40 \\
-1.71
\end{array}
$$

1. 182

19.466

1309.07

5.019

.124

.000

.976

$10.90(21.58)$ 


\section{APPENDIX H}

\section{MAXIMUM INCREMENTAL REACTIVITY}

ADJUSTMENT FACTORS (MIRs) ${ }^{(5)}$ 


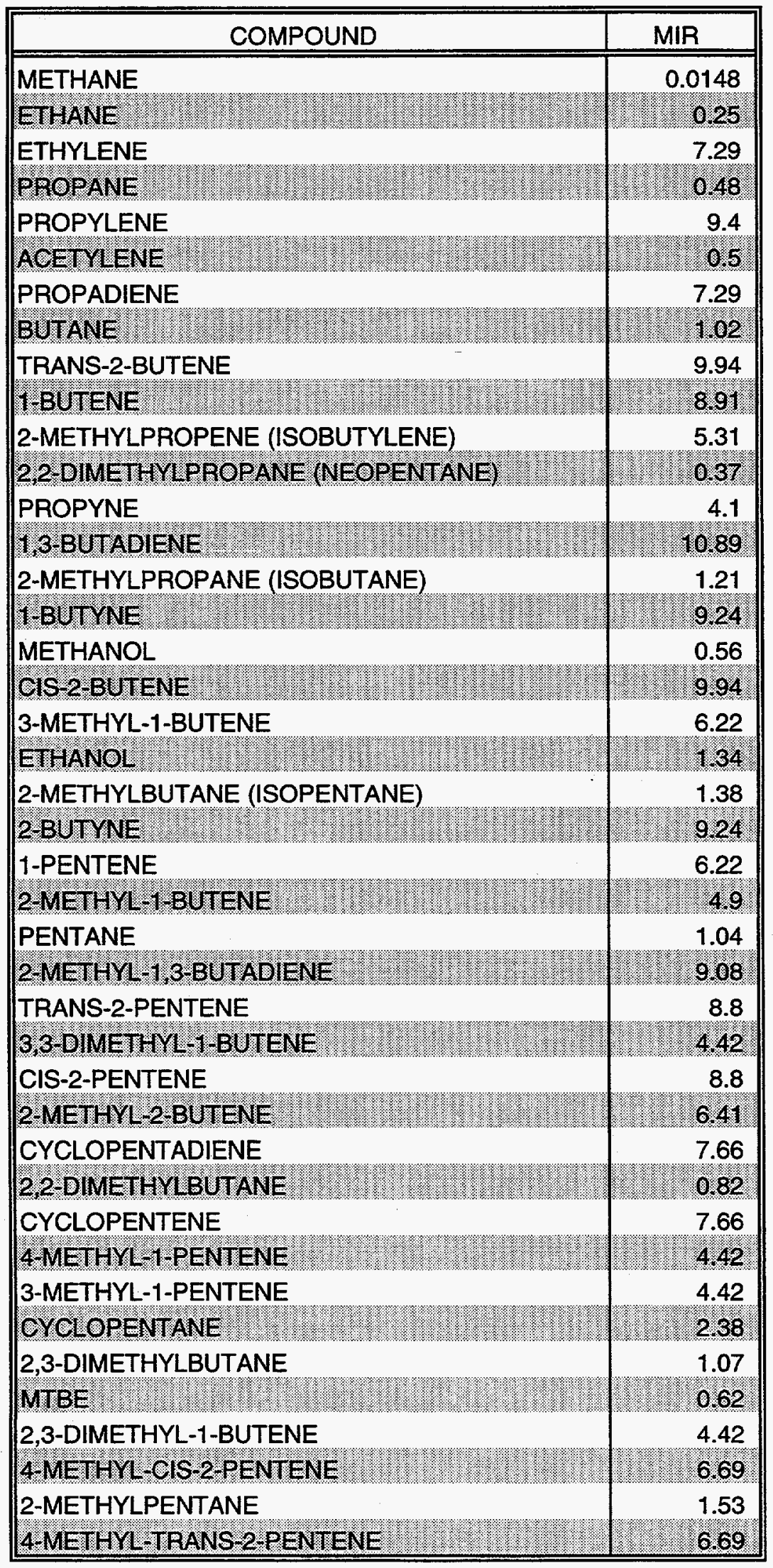




\begin{tabular}{|l|r|}
\hline \multicolumn{1}{|c|}{ COMPOUND } & \multicolumn{1}{|c|}{ MIR } \\
\hline \hline 3-METHYLPENTANE & 1.52 \\
2-METHYL-1-PENTENE & 4.42 \\
1-HEXENE & 4.42 \\
\hline HEXANE & 0.98 \\
TRANS-3-HEXENE & 6.69 \\
CIS-3HEXENE & 6.69 \\
TRANS-2-HEXENE & 6.69 \\
\hline 3-METHYL-TRANS-2-PENTENE & 6.69 \\
2-METHYL-2-PENTENE & 6.69 \\
3-METHYLYCLOPENTENE & 5.67 \\
CIS-2-HEXENE & 6.69 \\
\hline ETBE & 1.98 \\
3-METHYL-CIS-2-PENTENE & 6.69 \\
\hline 2,2-DIMETHYLPENTANE & 1.4 \\
METHYLCYCLOPENTANE & 2.82 \\
2,4-DIMETHYLPENTANE & 1.78 \\
2,3,3-TRIMETHYL-1-BUTENE & 3.48 \\
2,2,3-TRIMETHYLBUTANE & 1.32 \\
3,4-DIMETHYL-1-PENTENE & 3.48 \\
1-METHYLCYCLOPENTENE & 5.67 \\
BENZENE & 0.42 \\
3-METHYL-1-HEXENE & 3.48 \\
3,3-DIMETHYLPENTANE & 0.71 \\
CYCLOHEXANE & 1.28 \\
2-METHYLHEXANE & 1.08 \\
2,3-DIMETHYLENTANE & 1.51 \\
1,1-DIMETHYLYCLOPENTANE & 1.85 \\
CYCLOHEXENE & 5.67 \\
3-METHYLHEXANE & 1.4 \\
CIS-1,3-DIMETHYCYCLOPENTANE & 2.55 \\
3-ETHYLPENTANE & 1.4 \\
TRANS-1,2-DIMETHYCYCLOPENTANE & 1.85 \\
TRANS-1,3-DIMETHYLCYCLOPENTANE & 2.55 \\
1-HEPTENE & 3.48 \\
2,2,4-TRIMETHYLPENTANE & 0.93 \\
2-METHYL-1-HEXENE & 3.48 \\
TRANS-3-HEPTENE & 5.53 \\
HEPTANE & 0.81 \\
2-METHYL-2-HEXENE & 5.53 \\
3-METHYL-TRANS-3-HEXENE & 5.53 \\
TRANS-2-HEPTENE & 6.45 \\
3-ETHHL-CIS-2-PENTENE & \\
\hline
\end{tabular}




\begin{tabular}{|c|c|}
\hline COMPOUND & MIR \\
\hline 4,4-TRIMETHYL-1-PENTENE & 2.69 \\
\hline 2.4-TRIMETHYL-1-PENTENE & 2.69 \\
\hline 2,3-DIMETHYL-2-PENTENE & 5.53 \\
\hline IS-2-HEPTENE & 5.53 \\
\hline METHYLCYCLOHEXANE & 1.85 \\
\hline IS-1,2-DIMETHYLCYCLOPENTAN & 1.85 \\
\hline 2,2-DIMETHYLHEXANE & 1.2 \\
\hline 1, 1,3-TRIMETHYLCYCLOPENTAN & 1.94 \\
\hline 2,4,4-TRIMETHYL-2-PENTENE & 5.29 \\
\hline 2,2,3-TRIMETHYLPENTANE & 1.2 \\
\hline 2,5-DIMETHYLHEXANE & 1.63 \\
\hline ETHYLCYCLOPENTANE & 2.31 \\
\hline 2,4-DIMETHYLHEXANE & 1.5 \\
\hline 1-TRANS-2-CIS-4-TRIMETHYLCYCL & 1.94 \\
\hline 3,3-DIMETHYLHEXANE & 1.2 \\
\hline 1-TRANS-2-CIS-3-TRIMETHYLCYCL & 194 \\
\hline 2,3,4-TRIMETHYLPENTANE & 1.6 \\
\hline 2,3,3-TRIMETHYLPENTANE & 1.2 \\
\hline TOLUENE & 2.73 \\
\hline 2,3-DIMETHYLHEXANE & 1.32 \\
\hline 1,1,2-TRIMETHYLCYCLOPENTANE & 1.94 \\
\hline 2-METHYLHEPTANE & 0.96 \\
\hline 3,4-DIMETHYLHEXANE & 1.2 \\
\hline 2,2,4,4-TETRAMETHYLPENTANE & 1.14 \\
\hline 4-METHYLHEPTANE & 1.2 \\
\hline 2-METHYL-3-ETHYLPENTANE & 1.2 \\
\hline 2,6-DIMETHYLHEPTANE & 1.14 \\
\hline 3-METHYLHEPTANE & 0.99 \\
\hline 1-CIS,2-TRANS,3-TRIMETHYLCYCLOPENTANE & 1.94 \\
\hline CIS-1,3-DIMETHYLCYCLOHEXANE & 1.94 \\
\hline TRANS-1,4-DIMETHYLCYCLOHEXANE & 1.94 \\
\hline 3-ETHYLHEXANE & 1.2 \\
\hline 2,2,5-TRMMTHYLHEXANE & 0.97 \\
\hline CIS-1-METHYL-3-ETHYLCYCLOPENTANE & 1.94 \\
\hline 1.1-DIMETHYLCYCLOHEXANE & 1.94 \\
\hline TRANS-1-METHYL-2-ETHYLCYCLOPENTANE & 1.94 \\
\hline 1-METHY-1-ETHYL-CYCLOPENTANE & 1.94 \\
\hline 2,4,4-TRIMETHYLHEXANE & 1.14 \\
\hline 2,2,4-TRIMETHYLHEXANE & 1.14 \\
\hline TRANS-1,2-DIMETHYLCYCLOHEXANE & 1.94 \\
\hline & 69 \\
\hline TRANS-4-OCTENE & \\
\hline
\end{tabular}




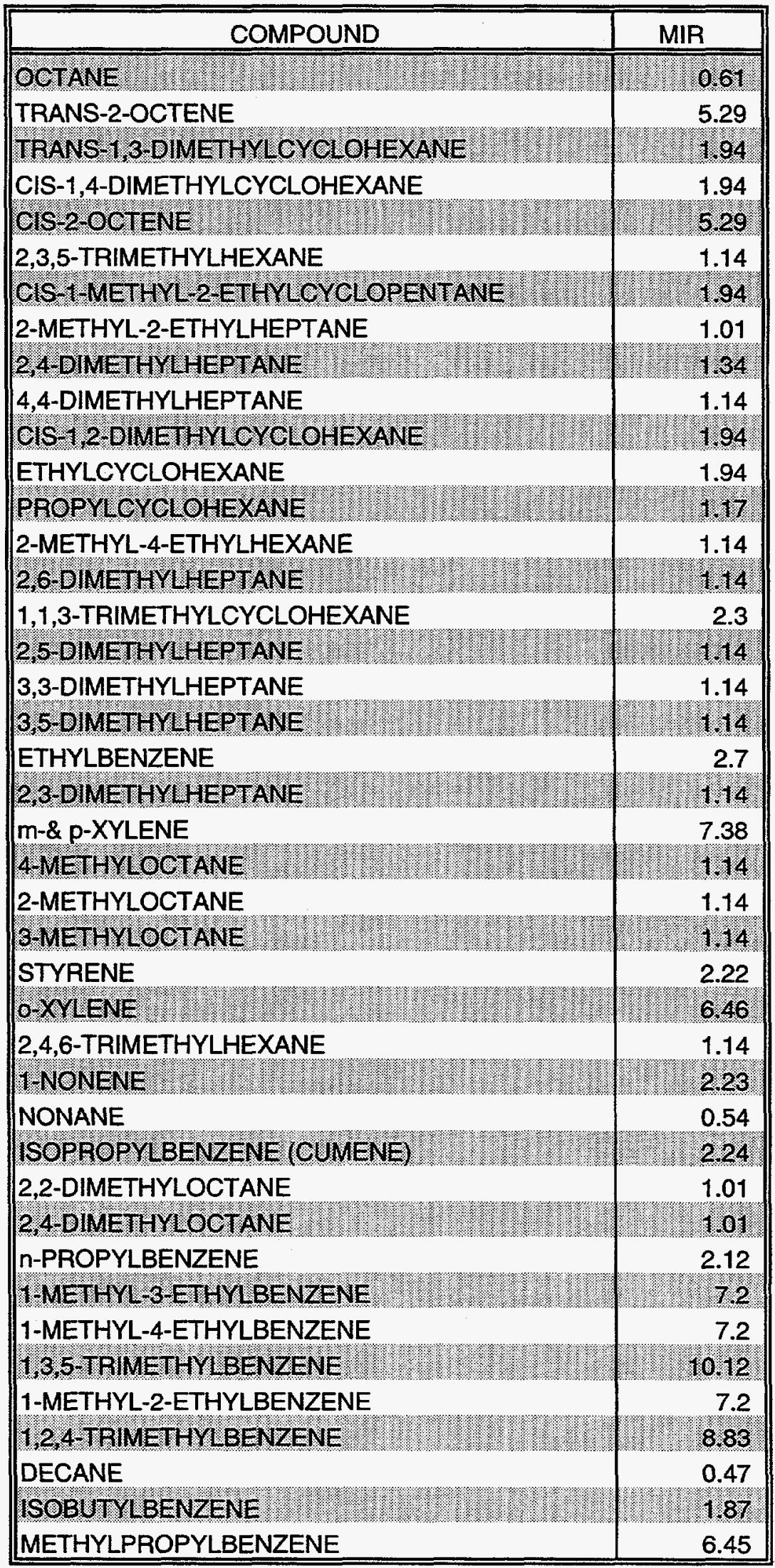




\begin{tabular}{|c|c|}
\hline COMPOUND & MIR \\
\hline S-BUTYLBENZEENE & 1.89 \\
\hline 1-METHYL-3-ISOPROPYLBENZENE & 5.84 \\
\hline 1,2,3-TRIMETHYLBENZENE & 8.85 \\
\hline 1-METHYL-4-ISOPROPYLBENZENE & 5.84 \\
\hline INDAN & 1.06 \\
\hline 1-METHYL-2-ISOPROPYLBENZENE & 5.84 \\
\hline 13. DIETHYLBENZENE & 6.45 \\
\hline 1,4-DIETHYLBENZENE & 6.45 \\
\hline 1-METHYL-3-N-PROPYLBENZENE & 6.45 \\
\hline 1-METHYL-4-N-PROPYLBENZENE & 6.45 \\
\hline 1,2 DIETHYLBENZENE & 6.45 \\
\hline 1-METHYL-2-N-PROPYLBENZENE & 6.45 \\
\hline 1.4-DIMETHYL-2-ETHYLBENZENE & 9.07 \\
\hline 1,3-DIMETHYL-4-ETHYLBENZENE & 9.07 \\
\hline 1,2-DIMETHYL-4-ETHYLBENZENE & 9.07 \\
\hline 1,3-DIMETHYL-2-ETHYLBENZENE & 9.07 \\
\hline UNDECANE: & 0.42 \\
\hline 1,2-DIMETHYL-3-ETHYLBENZENE & 9.07 \\
\hline 1,2,4.5-TETRAMETHYLBENZENE & 9.07 \\
\hline 2-METHYLBUTYLBENZENE (sec AN & 1.7 \\
\hline 1,2,3,5-TETRAMETHYBENZENE & 9.07 \\
\hline TERT-1-BUT-2-METHYLBENZENE & 5.84 \\
\hline 1,2,3,4-TETRAMETHYLBENZENE & 9.07 \\
\hline N-PENT-BENZENE & 1.7 \\
\hline TERT-1-BUT-3,5-DIMETHYLBENZENE & 7.5 \\
\hline NAPHTHALENE & 1.18 \\
\hline DODECANE & 0.38 \\
\hline FORMALDEHYDE & 7.15 \\
\hline ACETALDEHYDE & 5.52 \\
\hline ACROLEIN & 6.77 \\
\hline ACETONE & 0.56 \\
\hline PROPIONALDEHYDE & 6.53 \\
\hline CROTONALDEHYDE & 5.42 \\
\hline ISOBUTYRALDEHYDE & 5.26 \\
\hline METHYL ETHYL KETONE & 1.18 \\
\hline BENZALDEHYDE & -0.55 \\
\hline HEXANALDEHYDE & 3.79 \\
\hline
\end{tabular}




\section{APPENDIX I}

\section{AVERAGE SPECIATED EMISSIONS RESULTS}

FROM FTPs WITH LPG 


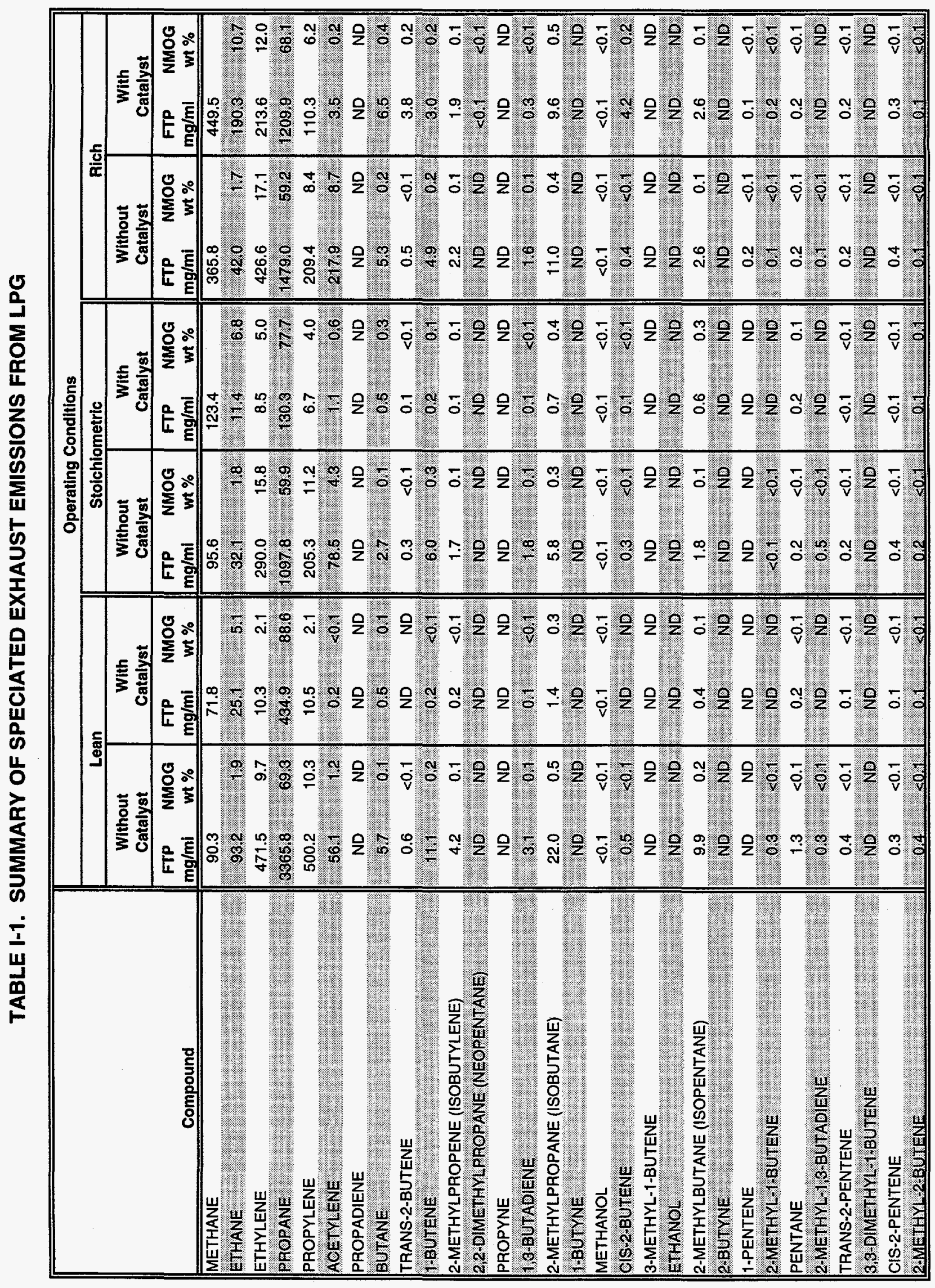


TABLE I-1 (CONT'D). SUMMARY OF SPECIATED EXHAUST EMISSIONS FROM LPG

\begin{tabular}{|c|c|c|c|c|c|c|c|c|c|c|c|c|}
\hline \multirow[b]{4}{*}{ Compound } & \multicolumn{12}{|c|}{ Operating Conditions } \\
\hline & \multicolumn{4}{|c|}{ Lean } & \multicolumn{4}{|c|}{ Stolchlometric } & \multicolumn{4}{|c|}{ Rich } \\
\hline & \multicolumn{2}{|c|}{$\begin{array}{l}\text { Without } \\
\text { Catalyst }\end{array}$} & \multicolumn{2}{|c|}{$\begin{array}{c}\text { With } \\
\text { Catalyst }\end{array}$} & \multicolumn{2}{|c|}{$\begin{array}{l}\text { Without } \\
\text { Catalyst }\end{array}$} & \multicolumn{2}{|c|}{$\begin{array}{c}\text { With } \\
\text { Catalyst }\end{array}$} & \multicolumn{2}{|c|}{$\begin{array}{l}\text { Without } \\
\text { Catalyst }\end{array}$} & \multicolumn{2}{|c|}{$\begin{array}{c}\text { With } \\
\text { Catalyst }\end{array}$} \\
\hline & $\begin{array}{c}\text { FTP } \\
\mathrm{mg} / \mathrm{mi}\end{array}$ & $\begin{array}{c}\text { NMOG } \\
\text { wt \% }\end{array}$ & $\begin{array}{c}\text { FTP } \\
\mathrm{mg} / \mathrm{mi}\end{array}$ & $\begin{array}{c}\text { NMOG } \\
\text { wt \% }\end{array}$ & $\begin{array}{c}\mathrm{FTP} \\
\mathrm{mg} / \mathrm{ml}\end{array}$ & $\begin{array}{c}\text { NMOG } \\
\text { wt } \%\end{array}$ & $\begin{array}{c}\text { FTP } \\
\mathrm{mg} / \mathrm{ml}\end{array}$ & $\begin{array}{c}\text { NMOG } \\
\text { wt \% } \\
\end{array}$ & $\begin{array}{c}\text { FTP } \\
\mathrm{mg} / \mathrm{mi}\end{array}$ & $\begin{array}{c}\text { NMOG } \\
\text { wt } \% \\
\end{array}$ & $\begin{array}{c}\text { FTP } \\
\mathrm{mg} / \mathrm{ml}\end{array}$ & $\begin{array}{c}\text { NMOG } \\
\text { wt \% } \\
\end{array}$ \\
\hline CYCLOPENTADIENE & 0.2 & $<0.1$ & ND & ND & $<0.1$ & $<0.1$ & ND & ND & 0.5 & $<0.1$ & 0.4 & $<0.1$ \\
\hline 2.2-DIMETHYLBUTANE & 0.5 & $=80.1=$ & $=0.4$ & $=0.1$ & 04 & 20.13 & 02 & 0.1 & 0.1 & $<01$ & 0.5 & -20.1 \\
\hline CYCLOPENTENE & 0.1 & $<0.1$ & ND: & ND & ND & ND & ND & ND & ND & ND & 0.1 & $<0.1$ \\
\hline 4.METH YL-T PENIENE & 0.1 & 201 & ND. & ND & No & ND & $\mathrm{No}$ & ND & 201 & $2.2 \times 0.1$ & -0.1 & 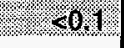 \\
\hline 3-METHYL-1-PENTENE & ND & ND & ND & ND & ND & ND & ND & ND & ND & ND & $<0.1$ & $<0.1$ \\
\hline CVCLOPENTANE & ND & NE. & ND & ND & $\mathrm{NO}$ & NO & ND & ND & ND & No & ND: & ND \\
\hline 2,3-DIMETHYLBUTANE & 0.6 & $<0.1$ & $<0.1$ & $<0.1$ & 0.2 & $<0.1$ & 0.1 & 0.1 & 0.3 & $<0.1$ & 0.1 & $<0.1$ \\
\hline MTBE & No & No & No & ND: & NB & NB & No & ND & No & ND & No: & No \\
\hline 2,3-DIMETHYL-1-BUTENE & ND & ND & ND & ND & ND & ND & ND & ND & ND & ND & ND & ND \\
\hline 4.METHYL CIS 2 PENTENE & ND. & 1.10 & $=\mathrm{NO}$ & NO. & ND & $1.2 \mathrm{ND}$ & No & 1.10 & NO & NO. & $\mathrm{NB}$ & Non \\
\hline 2-METHYLPENTANE & 1.1 & $<0.1$ & $<0.1$ & $<0.1$ & 0.7 & $<0.1$ & 0.1 & $<0.1$ & 0.3 & $<0.1$ & 0.2 & $<0.1$ \\
\hline 4-METHYL-TRANS 2-PENTENE & No & ND & ND & ND & ND & NB & NB. & $\mathrm{ND}$ & NO & ND. & No & ND. \\
\hline 3-METHYLPENTANE & 0.3 & $<0.1$ & 0.1 & $<0.1$ & 0.5 & $<0.1$ & 0.2 & 0.1 & 0.1 & $<0.1$ & 0.4 & $<0.1$ \\
\hline 2.METHYL T.PENTENE & NO & NO & NB & ND & No & NB & NB & 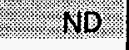 & NB & $\mathrm{NB}$ & 3. No & ND \\
\hline 1-HEXENE & 0.2 & $<0.1$ & ND & ND & 0.2 & $<0.1$ & 0.1 & $<0.1$ & 0.1 & $<0.1$ & 0.1 & $<0.1$ \\
\hline HEXANE : & $=0.1$ & 20.1 & $=80.1$ & 2.20 .1 & 20.7 & -201 & 0.1 & 0.11 & 0.1 & $<0.1$ & 0.1 & 20.1 \\
\hline TRANS-3-HEXENE & ND & ND & ND & ND & ND & ND & ND & ND & ND & ND & $<0.1$ & $<0.1$ \\
\hline OIS-8HEXENE & NO & ND & ND & NB & ND. & No & ND. & ND & ND. & No & 01 & 20.1 \\
\hline TRANS-2-HEXENE & ND & ND & ND & ND & ND & ND & ND & ND & ND & ND & ND & ND \\
\hline 6-METHML-TRANS 2 - PENTENE & 2.8 .1 & $2.80,1$ & 1.10 & No. & 20.1 & 20.1 & 20,1 & 20.1 & 20.1 & 40.1 & - 0.1 & 20.1 \\
\hline 2-METHYL-2-PENTENE & ND & ND & ND & ND & ND & ND & ND & ND & ND & ND & $<0.1$ & $<0.1$ \\
\hline BMETHYLYCLOPENTENE & $\mathrm{NB}$ & No & ND & No & ND & NB & NO & ND & ND & NB. & No & NO \\
\hline CIS-2-HEXENE & ND & ND & ND & ND & ND & ND & ND & ND & ND & ND & 0.1 & $<0.1$ \\
\hline ETBE & ND & NB & $=\mathrm{NB}$ & NO & ND. & ND & ND & ND & NB & No & ND & ND \\
\hline 3-METHYL-CIS-2-PENTENE & ND & ND & ND & ND & $<0.1$ & $<0.1$ & ND & ND & 0.1 & $<0.1$ & 0.1 & $<0.1$ \\
\hline 2. 2 DIMETHYLPENTANE & & 20. & 1.2. 20.1 & $<0.1$ & (20.1: & $20.1 \%$ & 20.1 & 20.1 & 0.5 & 20.11 & 0.1 & $<0,1$ \\
\hline METHYLCYCLOPENTANE & 0.2 & $<0.1$ & $<0.1$ & $<0.1$ & 0.2 & $<0.1$ & ND & ND & 0.2 & $<0.1$ & ND & ND \\
\hline 2.4 DDMETHYMPENTANE & 0.3 & 20.1 & 0.1 & $<0.1$ & 0.2 & .20 .17 & 20.1 & 40.1 & 0.2 & 20.1 & 0. & 20.1. \\
\hline 2,3,3-TRIMETHYL-1-BUTENE & ND & ND & ND & ND & ND & ND & ND & ND & ND & ND & ND & ND \\
\hline (2,2,32TRIMETH Y YBUTANE & $1 . \mathrm{NB}$ & $N N_{0}$ & $6: \mathrm{ND}=$ & No2. & MD & $\mathrm{NO}$ & 201 & 20.1. & 0.1 & $-80,4$ & 2.801 & 80.1 \\
\hline
\end{tabular}


TABLE I-1 (CONT'D). SUMMARY OF SPECIATED EXHAUST EMISSIONS FROM LPG

\begin{tabular}{|c|c|c|c|c|c|c|c|c|c|c|c|c|}
\hline \multirow[b]{4}{*}{ Compound } & \multicolumn{12}{|c|}{ Operating Conditions } \\
\hline & \multicolumn{4}{|c|}{ Lean } & \multicolumn{4}{|c|}{ Stolchlometric } & \multicolumn{4}{|c|}{ Rich } \\
\hline & \multicolumn{2}{|c|}{$\begin{array}{l}\text { Without } \\
\text { Catalyst }\end{array}$} & \multicolumn{2}{|c|}{$\begin{array}{c}\text { With } \\
\text { Catalyst }\end{array}$} & \multicolumn{2}{|c|}{$\begin{array}{l}\text { Without } \\
\text { Catalyst }\end{array}$} & \multicolumn{2}{|c|}{$\begin{array}{c}\text { With } \\
\text { Catalyst }\end{array}$} & \multicolumn{2}{|c|}{$\begin{array}{l}\text { Without } \\
\text { Catalyst }\end{array}$} & \multicolumn{2}{|c|}{$\begin{array}{c}\text { With } \\
\text { Catalyst }\end{array}$} \\
\hline & $\begin{array}{c}\text { FTP } \\
\mathrm{mg} / \mathrm{mi}\end{array}$ & $\begin{array}{c}\text { NMOG } \\
\text { wt \% }\end{array}$ & $\begin{array}{c}\text { FTP } \\
\mathrm{mg} / \mathrm{mi}\end{array}$ & $\begin{array}{c}\text { NMOG } \\
\text { wt \% }\end{array}$ & $\begin{array}{c}\text { FTP } \\
\mathrm{mg} / \mathrm{mi}\end{array}$ & $\begin{array}{l}\text { NMOG } \\
\text { wt \% }\end{array}$ & $\begin{array}{c}\text { FTP } \\
\mathrm{mg} / \mathrm{mi}\end{array}$ & $\begin{array}{c}\text { NMOG } \\
w t \%\end{array}$ & $\begin{array}{c}\text { FTP } \\
\mathrm{mg} / \mathrm{mi}\end{array}$ & $\begin{array}{c}\text { NMOG } \\
\text { wt } \%\end{array}$ & $\begin{array}{c}\text { FTP } \\
\mathrm{mg} / \mathrm{mi}\end{array}$ & $\begin{array}{c}\text { NMOG } \\
\text { wt \% }\end{array}$ \\
\hline 3,4-DIMETHYL-1-PENTENE & ND & $\overline{N D}$ & ND & $\overline{\mathrm{ND}}$ & ND & $\overline{N D}$ & ND & ND & ND & ND & ND & ND \\
\hline 1LMETHYLCYCLOPENTENE & ND & NB & ND & No & NO & ND $=$ & ND & ND & No. & No & 0.1 & 201 \\
\hline BENZENE & 0.7 & $<0.1$ & ND & ND & 0.6 & $<0.1$ & 0.1 & 0.1 & 0.7 & $<0.1$ & 3.5 & 0.2 \\
\hline 3 METHYL- T HEXENE & $\mathrm{ND}$ & .0 .10 & No & ND. & $=\mathrm{ND}$ & ND. & No: & No & No & ND & ND & No \\
\hline 3,3-DIMETHYLPENTANE & ND & ND & ND. & ND & ND & ND & ND & ND & $<0.1$ & $<0.1$ & 0.2 & $<0.1$ \\
\hline CVCLOHEXANE: & No & ND & ND & No & No & No & No & ND & $N D$ & NO & 201 & $\times 0.1$ \\
\hline 2-METHYLHEXANE & ND & ND & ND & ND & ND & ND & ND & ND & ND & ND & ND & ND \\
\hline 2,3.DIMETHYLPENTANE & 0.5 & $(201$. & 0.1 & . & 201 & $=-80.1$ ? & $<0: 1$ & $20.1 \%$ & 0.1 & $\times 0.1$ & 0.3. & 20.1 \\
\hline 1,1-DIMETHYLCYCLOPENTANE & ND & ND & ND & ND & ND & ND & ND & ND & ND & ND & ND & ND \\
\hline CYOLOHEXENE & ND & .101 & NB: & ND & ND & No & ND & $\mathrm{ND}$ & 02 & 20.0 & NO & ND \\
\hline 3-METHYLHEXANE & 0.1 & $<0.1$ & $<0.1$ & $<0.1$ & 0.1 & $<0.1$ & $<0.1$ & $<0.1$ & 0.1 & $<0.1$ & $<0.1$ & $<0.1$ \\
\hline CIS - 1, 3-DIMETHYLCYCLOPENTAN & ND & 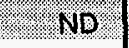 & ND. & 1.. ND. & $<0.1$ & $=(0.1$ & ND & ND & (6.). & 201 & $=2 .<1$ & 60.1 \\
\hline 3-ETHYLPENTANE & ND & ND & ND & ND & ND & ND & ND & ND & ND & ND & ND & ND \\
\hline TRANS 12 DIMETHYLCVCLOPEI & $\mathrm{NB}$ & No. & ND & ND. & NB & NB & No & NO. & NB & ND. & ND & No \\
\hline TRANS-1,3-DIMETHYLCYCLOPEN & ND & ND & ND & ND & ND & ND & ND & ND & ND & ND & ND & ND \\
\hline HHEPTENE : : & No & NB & ND & ND. & ND. & No & NB & No & No & NB & Ne & No \\
\hline 2,2,4-TRIMETHYLPENTANE & 0.6 & $<0.1$ & 0.1 & $<0.1$ & 0.4 & $<0.1$ & 0.3 & 0.2 & 0.2 & $<0.1$ & 0.6 & $<0.1$ \\
\hline 2.METHYL-1.YEXENE & ND & No & ND & NB & NO & ND & No & ND & NO & $\mathrm{ND}$ & No & No \\
\hline TRANS-3-HEPTENE & ND & ND & ND & ND & ND & ND & ND & ND & ND & ND & ND & ND \\
\hline GEPTANE & 2.201 & .20 .1 & .20 .1 & .011 & $0 \times 2$ & 20.1 & 0.1 & 20.1 . & 86.1 & 201 & 02 & 20.1 \\
\hline 2-METHYL-2-HEXENE & ND & ND & ND & ND & ND & ND & ND & ND & ND & ND & ND & ND \\
\hline 3METHVL-TRANS 3 HEXENE & ND & NB & . ND & ND. & ND & $1 . \mathrm{NO}$ & No & No & ND & ND & ND & No \\
\hline TRANS-2-HEPTENE & ND & ND & ND & ND & ND & ND & ND & ND & ND. & ND & ND & ND \\
\hline BETHYL-CIS, 2 ENTENE & ND. & No & No & NDi. & $\mathrm{ND}$ & ND & ND & No & 48 & ND & ND & ND \\
\hline 2,4,4-TRIMETHYL-1-PENTENE & 1.5 & $<0.1$ & ND & ND & 0.6 & $<0.1$ & ND & ND & $<0.1$ & $<0.1$ & ND & ND \\
\hline 2,2,4-TRIMETHVL 1. PENTENE & ND & NO & NB. & NB: & ND. & No & No & No & NB & NO & ND. & No. \\
\hline 2,3-DIMETHYL-2-PENTENE & ND & ND & ND & ND & ND & ND & ND & ND & ND & ND & ND & ND \\
\hline CIS.2. HEPTENE: & No & $N_{0}$ & NB & NB & ND & $\mathrm{NO}$ & No & ND & No: & NO & 01 & 80.1 \\
\hline METHYLCYCLOHEXANE & ND & ND & ND & ND & ND & ND & ND & ND & ND & ND & ND & ND \\
\hline 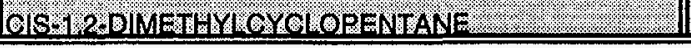 & $\mathrm{ND}$ & Non & ND & No & No & No & NO2 & Nin & ND & $\mathrm{No}$ & $\mathrm{Nob}$ & Nat \\
\hline
\end{tabular}




\begin{tabular}{|c|c|c|c|c|c|c|c|}
\hline \multicolumn{2}{|c|}{ MA } & पIN & (बIN & an & बN $=01 \%$ & पIN & \multirow{2}{*}{ 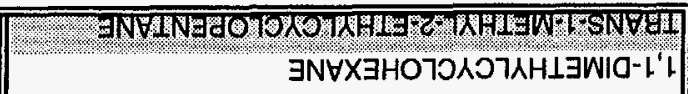 } \\
\hline aN & aN & aN & ON & ON & aN & aN & \\
\hline ON: & aN: & ON & $\mathrm{ON}$ & aN & aN. & $\mathrm{ON}$ & 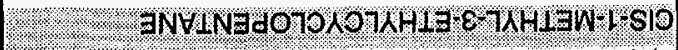 \\
\hline ON & ON & QN & aN & ON & an & ON & $\exists N \forall X \exists H 7 \wedge H \perp \exists W \mid \forall \perp-\varsigma^{\prime} Z^{\prime} Z$ \\
\hline aN & ON & GN: & aN & ON. $\mathrm{N}$ & ON. & बN. & 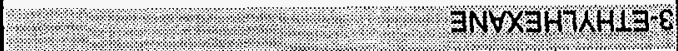 \\
\hline aN & aN & QN & an & ON & an & an & $\exists N \forall X \exists H O 7 J \wedge O 7 \wedge H \perp \exists W I Q \cdot t$-SNVYI \\
\hline on & on & oN & GN & $\mathrm{GN}$ & an: & an & 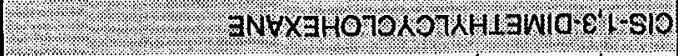 \\
\hline an & ON & QN & aN & ON & ON & ON & $\exists N \forall \perp N \exists d O 70 \Lambda 07 \wedge H \perp \exists W I Y \perp-\varepsilon S N \forall Y \perp-Z$ SIO- \\
\hline ON. & aN. & $10 \%: 20$ & (W) & $1.07 .910 \%$ & aN: : & $10 \%$ & 2. JN/AdA \\
\hline an & ON & ON & ON & aN & ON & ON & $\exists N \forall \perp d \exists H 7 \wedge H \perp \exists W I O-9^{\prime} Z$ \\
\hline $\mathrm{QN}$ & QN. & $\mathrm{ON}$ & aN & ON & aN: : aN & an & 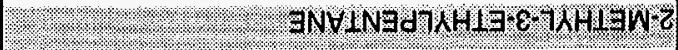 \\
\hline aN & aN & QN & an & ON & aN & aN & $\exists N \forall \perp d \exists H 7 \wedge H \perp \exists W-b$ \\
\hline an & ON. & ON. & बN & an & on & ON: & 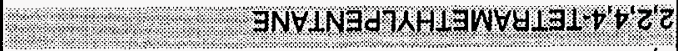 \\
\hline ON & QN & ON & ON & an & ON & an & $\exists N \forall X \exists H 7 \wedge H \perp \exists W I 0-b^{\prime} \varepsilon$ \\
\hline $\mathrm{ON}$ & ON & $\mathrm{ON} . \mathrm{aN}$ & GN. & $\mathrm{ON}$ & aN & ON. & 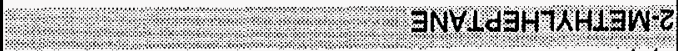 \\
\hline an & $\mathrm{aN}$ & aN & aN & aN & ON & $\mathrm{aN}$ & $\exists N \forall \perp N \exists d O 70 \wedge 07 \wedge H \perp \exists W I Y L-Z^{\prime} \iota^{\prime}$ \\
\hline GN: & बr. & $\mathrm{ON}=\mathrm{ON}$ & @N & av. & बN & aN: & FNWX \\
\hline 10 & 21 & $10>\quad \forall 0$ & 20 & $10>$ & $10>$ & $10>$ & $\exists \mathrm{N} \Xi \cap 701$ \\
\hline $10 s$ & 10 & 10 & ON & $10 \%$ & ON $: \mathrm{GN}$ & $10 \%$ & : aNGM \\
\hline aN & aN & an & GN & an & ON & an & $\exists N \forall \perp N \exists d] \wedge H \perp \exists W I Y \perp-\nabla^{\prime} \varepsilon^{\prime} Z$ \\
\hline $9 \mathrm{~N}$ & an & $\mathrm{aN}: \mathrm{aN}:$ & ON. & an $.9 N$ & $\mathrm{GN}: \mathrm{aN}$ & aN: : & 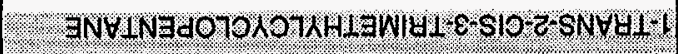 \\
\hline QN & ON & QN & ON & an & aN & aN & $\exists N \forall X \exists H 7 \wedge H \perp \exists W I 0-\varepsilon^{\prime} \varepsilon$ \\
\hline GN: & बN. & $\mathrm{an}$ & ON & an & $\mathrm{ON}=\mathrm{ON}=$ & ON: & 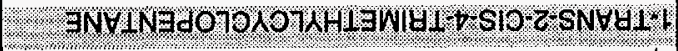 \\
\hline $10>$ & $\varepsilon 0$ & $1.0>$ & $10>$ & $10>$ & $\mathrm{aN}$ & $10>$ & $\exists N \forall X \exists H 7 \wedge H \perp \exists W I Q-\nabla^{\prime} Z$ \\
\hline an: & ON. & ars: & an & an. & ON: $: \mathrm{aN}$ : & an: an & 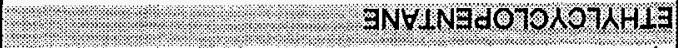 \\
\hline & QN & ON & ON & ON & $\mathrm{ON}$ & ON & 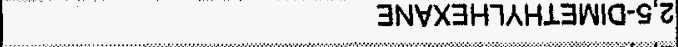 \\
\hline ON: & aN : & ON: & @N & $\mathrm{aN}$ & ON. & an & 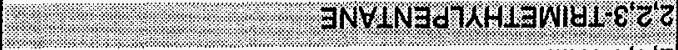 \\
\hline & $\mathrm{aN}$ & aN & aN & an & aN & $\mathrm{ON}$ & $\exists N \exists \perp N \exists d-Z-7 \lambda H \perp \exists W I Y \perp-b^{\prime} t^{\prime} z$ \\
\hline aN & GN. & aN: & ब1. & QN $=\mathrm{ON}$ & $0 \mathrm{~N} .=\mathrm{ON}$ & ON: & INVINA \\
\hline & aN & ON & an & $1.0>$ & $\mathrm{QN}$ & ON & 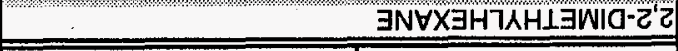 \\
\hline \%OWh & $\begin{array}{c}\mathrm{du} / \mathrm{\sigma W} \\
\mathrm{d} \perp \mathrm{y}\end{array}$ & $\begin{array}{cc}\% \text { IM } & \text { !W/6w } \\
\text { DOWN } & \text { dIJ }\end{array}$ & $\begin{array}{cc}\% \text { IM } & \text { !W } / 6 m \\
\text { DOWN } & \text { dLt }\end{array}$ & $\begin{array}{cc}\% m \text { Im/6um } \\
\text { vown dLt }\end{array}$ & $\begin{array}{cc}\% \text { IM } & \text { IU/bu } \\
\text { DOWN dL }\end{array}$ & $\begin{array}{cc}\% \text { IM } & \text { !L/LWU } \\
\text { DOWN } & \text { dLل }\end{array}$ & punodwos \\
\hline & & $\begin{array}{l}\text { I8KIEIES } \\
\text { InoYHM }\end{array}$ & $\begin{array}{l}18 \text { Kjejes } \\
\text { 41!M }\end{array}$ & 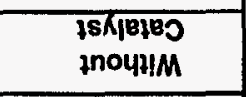 & 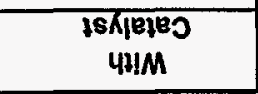 & $\begin{array}{l}\text { I8Xjejes } \\
\text { moyม!M }\end{array}$ & \\
\hline & $4=$ & & गामेणा & !़पए!०15 & & 807 & \\
\hline & & & 81 & jpesedo & & & \\
\hline
\end{tabular}

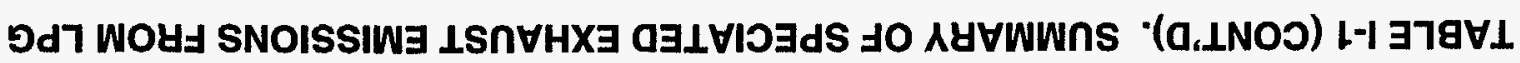


TABLE I-1 (CONT'D). SUMMARY OF SPECIATED EXHAUST EMISSIONS FROM LPG

\begin{tabular}{|c|c|c|c|c|c|c|c|c|c|c|c|c|}
\hline \multirow[b]{4}{*}{ Compound } & \multicolumn{12}{|c|}{ Operating Conditions } \\
\hline & \multicolumn{4}{|c|}{ Lean } & \multicolumn{4}{|c|}{ Stoichlometric } & \multicolumn{4}{|c|}{ Rich } \\
\hline & \multicolumn{2}{|c|}{$\begin{array}{l}\text { Without } \\
\text { Catalyst }\end{array}$} & \multicolumn{2}{|c|}{$\begin{array}{c}\text { With } \\
\text { Catalyst }\end{array}$} & \multicolumn{2}{|c|}{$\begin{array}{l}\text { Without } \\
\text { Catalyst }\end{array}$} & \multicolumn{2}{|c|}{$\begin{array}{c}\text { With } \\
\text { Catalyst }\end{array}$} & \multicolumn{2}{|c|}{$\begin{array}{l}\text { Without } \\
\text { Catalyst }\end{array}$} & \multicolumn{2}{|c|}{$\begin{array}{c}\text { With } \\
\text { Catalyst }\end{array}$} \\
\hline & $\begin{array}{l}\text { FTP } \\
\mathrm{mg} / \mathrm{mi}\end{array}$ & $\begin{array}{c}\text { NMOG } \\
\text { wt \% }\end{array}$ & $\begin{array}{c}\mathrm{FTP} \\
\mathrm{mg} / \mathrm{mi}\end{array}$ & $\begin{array}{c}\text { NMOG } \\
\text { wt \% }\end{array}$ & $\begin{array}{c}\text { FTP } \\
\mathrm{mg} / \mathrm{mi}\end{array}$ & $\begin{array}{c}\text { NMOG } \\
\text { wt \% }\end{array}$ & $\begin{array}{c}\text { FTP } \\
\mathrm{mg} / \mathrm{ml}\end{array}$ & $\begin{array}{c}\text { NMOG } \\
\text { wt \% }\end{array}$ & $\begin{array}{c}\text { FTP } \\
\mathrm{mg} / \mathrm{mi}\end{array}$ & $\begin{array}{c}\text { NMOG } \\
\text { wt \% }\end{array}$ & $\begin{array}{c}\text { FTP } \\
\mathrm{mg} / \mathrm{mi}\end{array}$ & $\begin{array}{c}\text { NMOG } \\
\text { wt \% }\end{array}$ \\
\hline 1-METHYL-1-ETHYL-CYCLOPENTA & ND & ND & ND & ND & ND & ND & ND. & ND & ND & ND & ND & ND \\
\hline 2.4.4 TRIMETHVUHEXANE & No & ND & ND & No & ND & No & No & ND & No & $\mathrm{NB}$ & NO & ND \\
\hline 2,2,4-TRIMETHYLHEXANE & ND & ND & ND & ND & ND & ND & ND & ND & ND & ND & ND & ND \\
\hline TRANS 1,2DIMETHVLOYCLOHEXANE & NB & $\mathrm{NB}$ & No & $=1.20$ & I. $\mathrm{ND}$ & ND. & No & $\mathrm{NO}$ & ND & No & Ne & NO \\
\hline 1-OCTENE & ND & ND & ND & ND & ND & ND & ND & ND & ND & ND & ND & ND \\
\hline TRANS 4 OCTENE & ND & No & ND & $\mathrm{ND}$ & $\mathrm{NO}$ & ND & No: & ND & $\mathrm{NB}$ & No & NB & No \\
\hline OCTANE & ND & ND & ND & ND & ND & ND & $<0.1$ & $<0.1$ & ND & ND & $<0.1$ & $<0.1$ \\
\hline TRANS 2 OOTENE & NO & $\mathrm{NO}$ & No & ND & ND & $.2 \mathrm{NO}$ & ND: & No & No. & ND & $\mathrm{ND}$ & No. \\
\hline TRANS-1,3-DIMETHYLCYCLOHEXANE & ND & ND & ND & ND & ND & ND & ND & ND & ND & ND & ND & ND \\
\hline CIS 1 4 DIMETHYL YOLOHEXANE & $N B$ & $\mathrm{NB}$ & No & . ND & $N D$ & NB & No & No & No) & No & ND & No \\
\hline CIS-2-OCTENE & ND & ND & ND & ND & ND & ND & ND & ND & ND & ND & ND & ND \\
\hline 2.3.5.TAIMETHYUEXANE & $\mathrm{NO}$ & No. & NB & ND. & No: & No. & . ND. & $\mathrm{NO}$ & ND: & No & ND. & NB \\
\hline CIS-1-METHYL-2-ETHYLCYCLOPEN & ND & ND & ND & ND & ND & ND & ND & ND & ND & ND & ND & ND \\
\hline 2.METHYL 2 ETHYLIEPTANE : & $\mathrm{NB}$ & No & No & No & No. & 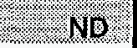 & No & No & NO & NDO & NB. & No \\
\hline 2,4-DIMETHYLHEPTANE & 0.4 & $<0.1$ & 0.6 & 0.1 & 0.2 & $<0.1$ & 0.7 & 0.4 & 0.4 & $<0.1$ & 0.7 & $<0.1$ \\
\hline 4.4DIMETHYLHEPTANE: & ND. & . ND. & UD & No. & ND & 1. $\mathrm{NB}$ & $\mathrm{NB}$ & NB & $\mathrm{NB}$ & ND & No & No \\
\hline CIS-1,2-DIMETHYLCYCLOHEXANE & ND & ND & ND & ND & ND & ND & ND & ND & ND & ND & ND & ND \\
\hline ETHYLOYOLOHEXANE & NB & ND: & No & No & NB & NE. & ND & No & INE & $\mathrm{NB}$ & Na: & No \\
\hline PROPYLCYCLOHEXANE & ND & ND & ND & ND & ND & ND & ND & ND & ND & ND & ND & ND \\
\hline 2. METHYL 4 ETHYMHEXANE & NE & No & ND. & $\mathrm{NO}$ & ND & NB & ND & NO & No & ND: & NB: & ND \\
\hline 2,6-DIMETHYLHEPTANE & ND & ND & ND & ND & ND & ND & ND & ND & ND & ND & ND & ND \\
\hline 1.1.3 TRIMETHYLCYCLOHEXANE: & NO: & No. & ND & No & NB & NB & ND & ND & No & 1.10 & NB & No \\
\hline 2,5-DIMETHYLHEPTANE & ND & ND & ND & ND & ND & ND & ND & ND & ND & ND & ND & ND \\
\hline 3,8DIMETH WHEPTANE . & NO & $N D$ & No & No & No & 1.10 & ND & ND. & No & No & 1. $1 \mathrm{~B}$ & $\mathrm{NB}$ \\
\hline 3,5-DIMETHYLHEPTANE & ND & ND & ND & ND & ND & ND & ND & ND & ND & ND & ND & ND \\
\hline ETHYLENZENE & 0.2 & $.20 .1 \%$ & 0.1 & 80.1 & 01 & .80 .4 & 0.1 & 20.1 & 02 & $(80.11$ & 0.2 & 20.1 \\
\hline 2,3-DIMETHYLHEPTANE & ND & ND & ND & ND & ND & ND & ND & ND & ND & ND & ND & ND \\
\hline m-8 p.XYLENE & .04 & $80.1:$. & 02 & 20.1 & & 20.1 & <0.1 & $20.1 \div$ & & 20.1 & 0.7. & $2<0,1$ \\
\hline 4-METHYLOCTANE & ND & ND & ND & ND & ND & ND & ND & ND & ND & ND & ND & ND \\
\hline 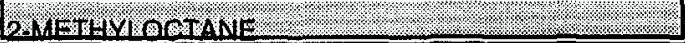 & $N$ & $N$ & 1.6 .110 & $\mathrm{Na}$ & Nin & $\sqrt{10}$ & 3. & 110. & ans & . & $\mathrm{NO}_{2}$ & 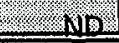 \\
\hline
\end{tabular}




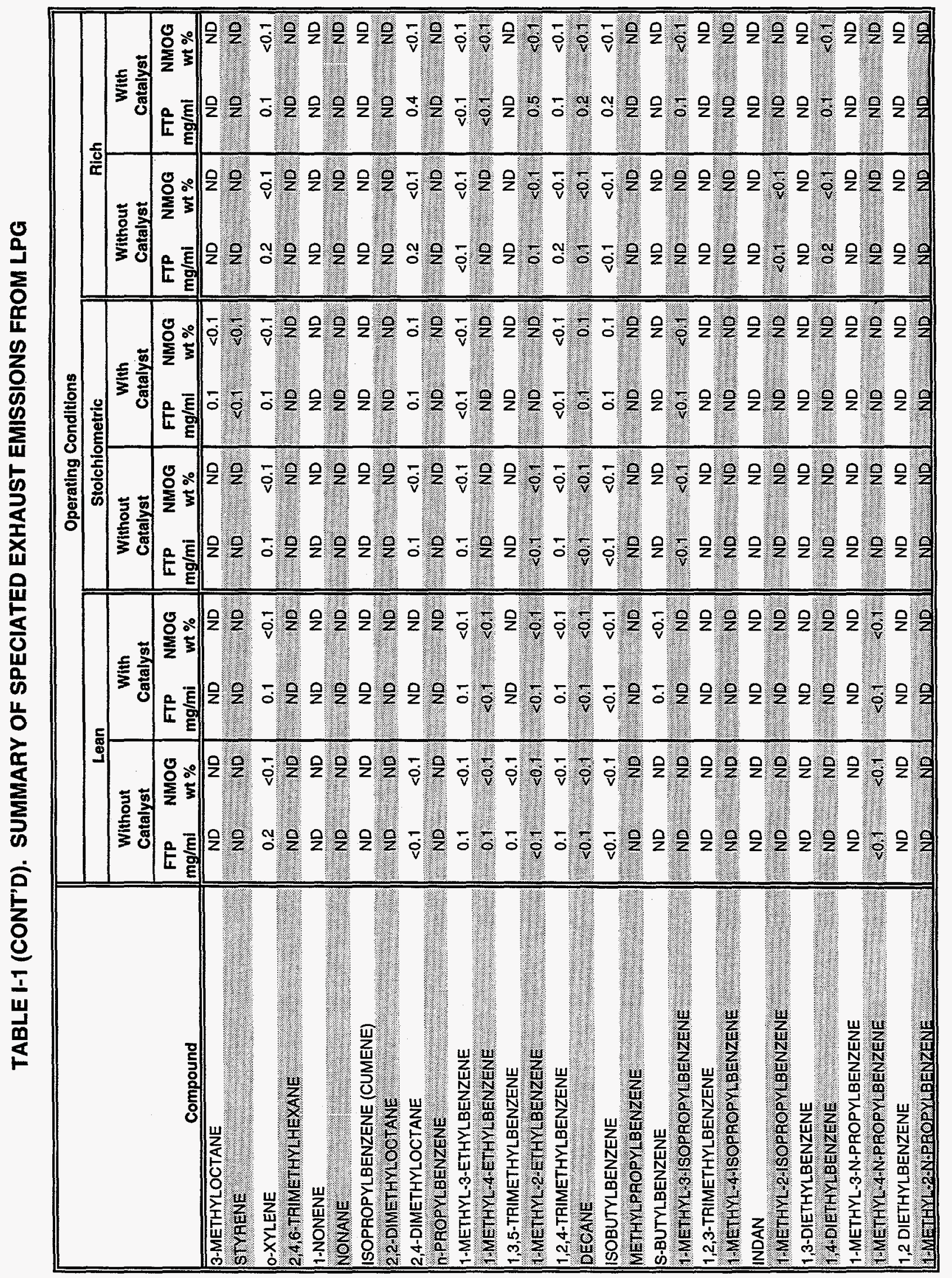


TABLE I-1 (CONT'D). SUMMARY OF SPECIATED EXHAUST EMISSIONS FROM LPG

\begin{tabular}{|c|c|c|c|c|c|c|c|c|c|c|c|c|}
\hline \multirow[b]{4}{*}{ Compound } & \multicolumn{12}{|c|}{ Operating Conditions } \\
\hline & \multicolumn{4}{|c|}{ Lean } & \multicolumn{4}{|c|}{ Stolchiometric } & \multicolumn{4}{|c|}{ Rich } \\
\hline & \multicolumn{2}{|c|}{$\begin{array}{l}\text { Without } \\
\text { Catalyst }\end{array}$} & \multicolumn{2}{|c|}{$\begin{array}{c}\text { With } \\
\text { Catalyst }\end{array}$} & \multicolumn{2}{|c|}{$\begin{array}{l}\text { Without } \\
\text { Catalyst }\end{array}$} & \multicolumn{2}{|c|}{$\begin{array}{c}\text { With } \\
\text { Catalyst }\end{array}$} & \multicolumn{2}{|c|}{$\begin{array}{l}\text { Without } \\
\text { Catalyst }\end{array}$} & \multicolumn{2}{|c|}{$\begin{array}{c}\text { With } \\
\text { Catalyst }\end{array}$} \\
\hline & $\begin{array}{c}\text { FTP } \\
\mathrm{mg} / \mathrm{mi}\end{array}$ & $\begin{array}{c}\text { NMOG } \\
w t \% \\
\end{array}$ & $\begin{array}{c}\text { FTP } \\
\mathrm{mg} / \mathrm{mi}\end{array}$ & $\begin{array}{c}\text { NMOG } \\
\text { wt \% } \\
\end{array}$ & $\begin{array}{c}\text { FTP } \\
\mathrm{mg} / \mathrm{mi}\end{array}$ & $\begin{array}{c}\text { NMOG } \\
w t \% \\
\end{array}$ & $\begin{array}{c}\text { FTP } \\
\mathrm{mg} / \mathrm{mi}\end{array}$ & $\begin{array}{c}\text { NMOG } \\
\text { Wt \% }\end{array}$ & $\begin{array}{c}\text { FTP } \\
\mathrm{mg} / \mathrm{mi}\end{array}$ & $\begin{array}{c}\text { NMOG } \\
\text { wt \% } \\
\end{array}$ & $\begin{array}{c}\text { FTP } \\
\mathrm{mg} / \mathrm{mi}\end{array}$ & $\begin{array}{c}\text { NMOG } \\
\text { wt \% } \\
\end{array}$ \\
\hline 1,4-DIMETHYL-2-ETHYLBENZENE & $<0.1$ & $<0.1$ & ND & ND & ND & ND & ND & ND & ND & ND & ND & ND \\
\hline 1.3.DIMETHYL-4-ETHYLBENZENE & ND & No. & ND & ND & $\mathrm{ND}$ & No & NB & ND & NB & ND. & ND. & ND \\
\hline 1,2-DIMETHYL-4-ETHYLBENZENE & $<0.1$ & $<0.1$ & ND & ND & $<0.1$ & $<0.1$ & ND & ND & 0.1 & $<0.1$ & $<0.1$ & $<0.1$ \\
\hline 1 3.DIMETHYL-2ETHYLBENZENE. & ND. & $\mathrm{NO}$ & No & No & No & No. & ND & NB & No & ND & $\mathrm{ND}$ & NO \\
\hline UNDECANE & ND & ND & ND & ND & 0.1 & $<0.1$ & 0.1 & $<0.1$ & $<0.1$ & $<0.1$ & 0.1 & $<0.1$ \\
\hline 1.2-DIMETHVL-3-ETHYLEENZENE & No & No. & ND & $\mathrm{NO}$ & $\mathrm{ND}$ & $\mathrm{NB}$ & $\mathrm{NB}$ & No. & No & No & No & $=.10$ \\
\hline 1,2,4,5-TETRAMETHYLBENZENE & ND & ND & 0.2 & $<0.1$ & ND & ND & ND & ND & ND & ND & ND & ND \\
\hline 2-METHYLBUTYLBENZENE (SOC AMYLBENZENE) & NB & No & No & No & NB & ND & No & No & No & ND. & ND & NO \\
\hline 3,4 DIMETHYLCUMENE & ND & ND & ND & ND & ND & ND & ND & ND & ND & ND & ND & ND \\
\hline (1,2,3,5.TETRAMETHY BENZENE & No & $1.2 \mathrm{~N}$ & No: & No: & ND & No & ND & No & No & No & No & No \\
\hline TERT-1-BUT-2-METHYLBENZENE & ND & ND & ND & ND & ND & ND & ND & ND & ND & ND & ND & ND \\
\hline $1,2.3,4.1 E T R A M E T H Y L B E N Z E N E$ & ND & No & ND. & NO. & NB & ND. & NB & NO & No: & No. & Ne & 1.20 \\
\hline N-PENT-BENZENE & ND & ND & ND & ND & ND & ND & ND & ND & 0.1 & $<0.1$ & ND & ND \\
\hline TERT-1 BUT 3 S S-DIMETHYLBENZEN & $\mathrm{NB}$ & ND & ND. & NO. & No & ND & ND. & $\mathrm{ND}$ & ND & No & No. & ND. \\
\hline NAPHTHALENE & ND & ND & ND & ND & ND & ND & ND & ND & ND & ND & ND & ND \\
\hline DODECANE: & $\mathrm{NB}$ & $\mathrm{NB}$ & No & No & 0.1 & .0 .11 & 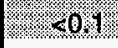 & .0 .1 & 0.1. & $(20.1$ & NE & No \\
\hline FORMALDEHYDE & 199.8 & 4.1 & 2.4 & 0.5 & 53.1 & 2.9 & 1.0 & 0.6 & 52.7 & 2.1 & 1.6 & 0.1 \\
\hline ACETALDEHYDE & 38.9 & 0.8 & 0.7. & 0.1 & 177 & 1.0. & 1.04 & 0.2 & 10.3 & $0.4:$ & 12 & 0.1 \\
\hline ACROLEIN & 0.2 & $<0.1$ & ND & ND & 0.2 & $<0.1$ & ND & ND & ND & ND & ND & ND \\
\hline ACETONE & .0 .1 & ( 10.1 & 0.1 & 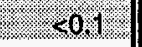 & $<0.1$ & $=20.1 \%$ & 0.1 & 0.1 & 0.1 & 20.1 & 0.1 & (20.1. \\
\hline PROPIONALDEHYDE & 0.1 & $<0.1$ & 0.1 & $<0.1$ & $<0.1$ & $<0.1$ & 0.1 & 0.1 & 0.1 . & $<0.1$ & 0.1 & $<0.1$ \\
\hline CROTONALBEHYDE & 20 & 20.1. & No & ND & .100 & No & ND. & NB & 80.1 & 20.1 . & . & No \\
\hline ISOBUTYRALDEHYDE & 0.1 & $<0.1$ & ND & ND & ND & ND & $<0.1$ & $<0.1$ & $<0.1$ & $<0.1$ & 0.2 & $<0.1$ \\
\hline METHVL ETHVL KETONE & No & No & ND & ND & No & No & NO & NB & ND & NB & NO & No \\
\hline BENZALDEHYDE & ND & $N D$ & ND & ND & ND & ND & ND & ND & ND & ND & ND & ND \\
\hline GEXANAI DEHYDE & NB & $\% \mathrm{NB}$ & ND. & No & No & ND & No & No & No & NB & No & No \\
\hline SUMMATION OF NONMETHANE COMPOUNDS & 4849.7 & 99.9 & 490.2 & 99.8 & 1825.4 & 99.6 & 166.6 & 99.4 & 2494.4 & 99.9 & 1776.1 & 99.9 \\
\hline
\end{tabular}




\section{APPENDIX J}

\section{AVERAGE SPECIATED EMISSIONS RESULTS FROM FTPs WITH CNG}


TABLE J-1. SUMMARY OF SPECIATED EXHAUST EMISSIONS FROM CNG

\begin{tabular}{|c|c|c|c|c|c|c|c|c|c|c|c|c|}
\hline \multirow[b]{4}{*}{ Compound } & \multicolumn{12}{|c|}{ Operating Conditions } \\
\hline & \multicolumn{4}{|c|}{ Lean } & \multicolumn{4}{|c|}{ Stoichiometric } & \multicolumn{4}{|c|}{ Rich } \\
\hline & \multicolumn{2}{|c|}{$\begin{array}{l}\text { Without } \\
\text { Catalyst }\end{array}$} & \multicolumn{2}{|c|}{$\begin{array}{c}\text { With } \\
\text { Catalyst }\end{array}$} & \multicolumn{2}{|c|}{$\begin{array}{l}\text { Without } \\
\text { Catalyst }\end{array}$} & \multicolumn{2}{|c|}{$\begin{array}{c}\text { With } \\
\text { Catalyst }\end{array}$} & \multicolumn{2}{|c|}{$\begin{array}{l}\text { Without } \\
\text { Catalyst }\end{array}$} & \multicolumn{2}{|c|}{$\begin{array}{c}\text { With } \\
\text { Catalyst }\end{array}$} \\
\hline & $\begin{array}{c}\text { FTP } \\
\mathrm{mg} / \mathrm{mi}\end{array}$ & $\begin{array}{c}\text { NMOG } \\
\text { wt \% }\end{array}$ & $\begin{array}{c}\text { FTP } \\
\mathrm{mg} / \mathrm{mi}\end{array}$ & $\begin{array}{c}\text { NMOG } \\
\text { wt \% }\end{array}$ & \begin{tabular}{|c} 
FTP \\
$\mathrm{mg} / \mathrm{mi}$
\end{tabular} & $\begin{array}{c}\text { NMOG } \\
\text { wt \% }\end{array}$ & $\begin{array}{c}\text { FTP } \\
\mathrm{mg} / \mathrm{mi}\end{array}$ & $\begin{array}{c}\text { NMOG } \\
\text { wt } \% \\
\end{array}$ & $\begin{array}{l}\text { FTP } \\
\mathrm{mg} / \mathrm{mi}\end{array}$ & $\begin{array}{c}\text { NMOG } \\
\text { wt \% }\end{array}$ & $\begin{array}{c}\text { FTP } \\
\mathrm{mg} / \mathrm{mi}\end{array}$ & $\begin{array}{l}\text { NMOG } \\
\text { wt \% }\end{array}$ \\
\hline METHANE & 2127.7 & & 964.3 & & 2688.0 & & 809.5 & & 10540.2 & & 8826.1 & \\
\hline ETHANE ? & 1397 & 30.8 & 41.8 & 68.5 & 208.2 & 38.3 & 272 & 60.9 & 708.8 & 39.2 & 290.5 & .653. \\
\hline ETHYLENE & 106.8 & 23.5 & 1.4 & 2.3 & 98.9 & 18.2 & 2.7 & 6.1 & 381.8 & 21.1 & 33.2 & 7.5 \\
\hline PBOPANE & .54 .3 & 120 & 110 & 18.0 & 94.3 & 17.4 & .72 & 16.1 & 3412 & .18 .9 & 908 & 20.4 \\
\hline PROPYLENE & 12.1 & 2.7 & 0.2 & 0.3 & 12.6 & 2.3 & 0.4 & 0.9 & 30.4 & 1.7 & 5.4 & 1.2 \\
\hline ACET YLENE & 257 & 57 & 0.1 & 0.2 & 27.5 & .6 .1 & 0.3 & 07. & 169.5 & 9.4 & 2.1 & 0.5 \\
\hline PROPADIENE & ND & ND & ND & ND & ND & ND & ND & ND & ND & ND & ND & ND \\
\hline BUTANE & 0.5 & .01. & 0.3 & 0.4 & 2.2 & 0.4 & 0.3 & 0.6 & 24 & 0.1 & 1.5 & 0.3 \\
\hline TRANS-2-BUTENE & 0.2 & 0.1 & $<0.1$ & 0.1 & 0.3 & $<0.1$ & $<0.1$ & 0.1 & 0.4 & $<0.1$ & $<0.1$ & $<0.1$ \\
\hline HBUTENE & 0.9 & 0.2 & ND & $=\mathrm{ND}$ & 0.8 & 1.02 & $0.1 \%$ & 0.2 & 10.0 & 0.1 & 0.1 & $<0.1$ \\
\hline 2-METHYLPROPENE (ISOBUTYLENE) & 1.5 & 0.3 & 0.1 & 0.2 & 1.6 & 0.3 & 0.1 & 0.1 & 3.4 & 0.2 & 0.3 & 0.1 \\
\hline 2:2-DIMETHYLPROPANE INEOPENTANE & 20.1 & 2.2011 & 20.1 & .201 & 0.1 & 201 & 20.1 & .0 .1 & 20.1 & $2: 20.1$ & 2.8 .1 & .20 .1 \\
\hline PROPYNE & ND & ND & ND & ND & ND & ND & ND & ND & ND & ND & ND & ND \\
\hline 1,3-BUTADIENE: & 0.8 & 0.2 & $<0.1$ & 20.1 & 0.6 & $0.1 \%$ & 0.1 & 0.3 & 0.044 & 2.20 .1 & $<0.1$. & 20.1 \\
\hline 2-METHYLPROPANE (ISOBUTANE) & 0.2 & $<0.1$ & $<0.1$ & $<0.1$ & 1.0 & 0.2 & $<0.1$ & 0.1 & 2.1 & 0.1 & 0.3 & 0.1 \\
\hline 1.BUTYNE & $=$ ND & NO. & NB & NB: & ND & ND. & ND & ND. & ND & No & ND. & ND. \\
\hline METHANOL & $<0.1$ & $<0.1$ & $<0.1$ & $<0.1$ & $<0.1$ & $<0.1$ & $<0.1$ & $<0.1$ & $<0.1$ & $<0.1$ & $<0.1$ & $<0.1$ \\
\hline CIS-2-BUTENE & 0.2 & $<0,1$ & 20.1 & 20.1 & 0.2 & $(.801$ & <. & 0.1 & 0.3. & 80.1 & 0.1 & $<0.1$ \\
\hline 3-METHYL-1-BUTENE & ND & ND & ND & ND & ND & ND & ND. & ND & ND & ND & ND & ND \\
\hline ETHANOL:?:? & ND & NE & No & No & NB & $1.2 \mathrm{NB}$ & ND: & ND & ND. & ND & NO & ND \\
\hline 2-METHYLBUTANE (ISOPENTANE) & 1.4 & 0.3 & 0.5 & 0.8 & 5.5 & 1.0 & 0.5 & 1.0 & 5.5 & 0.3 & 2.7 & 0.6 \\
\hline (2-BUTVNE & NO & ND & NO NO & NE: & NB & ND & $\mathrm{NO}$ & $\mathrm{NB}$ & $\mathrm{ND}$ & No & No & ND \\
\hline 1-PENTENE & 0.1 & $<0.1$ & $<0.1$ & $<0.1$ & 0.2 & $<0.1$ & $<0.1$ & $<0.1$ & 0.1 & $<0.1$ & 0.1 & $<0.1$ \\
\hline $2 M E T H Y L$ - 1-BUTENE. & 0.2 & $\times 0.1$ & 0.1 & 01. & 02 & $<0.1$ & .20 .1 & 6.120 .1 & 0.3 & 20.1 & 022 & 201 \\
\hline PENTANE & 0.2 & $<0.1$ & 0.1 & 0.2 & 0.6 & 0.1 & 0.1 & 0.2 & 0.7 & $<0.1$ & 1.8 & 0.4 \\
\hline 2-METHYL-1 3-BUTADIENE & 20.1 & 20.1 : & 0.1 & 0.1 & 0.2 & 20.7 & 201 & 0.1 .1 & .801 & 20.1 & 0.2 & $(2.80 .1$ \\
\hline TRANS-2-PENTENE & 0.1 & $<0.1$ & $<0.1$ & $<0.1$ & 0.1 & $<0.1$ & $<0.1$ & $<0.1$ & 0.2 & $<0.1$ & 0.3 & 0.1 \\
\hline 3,3.0IMETHYL-1-BUTENE & ND & ND. & .20. & NO & No & ND & ND & NB & ND & ND & NO & NB \\
\hline CIS-2-PENTENE & $<0.1$ & $<0.1$ & $<0.1$ & $<0.1$ & 0.1 & $<0.1$ & $<0.1$ & $<0.1$ & 0.2 & $<0.1$ & 0.1 & $<0.1$ \\
\hline 2.METHYL_.BUITENE & 0.3. & $2 . a_{1} \cdot 1$. & $=2.0 .1$. & $n_{0.1}$ & $=04$ & $n_{1}$ & 20.1 & $=0.4$. & 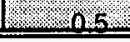 & 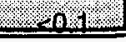 & 2.04 & 0.4 \\
\hline
\end{tabular}


TABLE J-1 (CONT'D). SUMMARY OF SPECIATED EXHAUST EMISSIONS FROM CNG

\begin{tabular}{|c|c|c|c|c|c|c|c|c|c|c|c|c|}
\hline \multirow[b]{4}{*}{ Compound } & \multicolumn{12}{|c|}{ Operating Conditions } \\
\hline & \multicolumn{4}{|c|}{ Lean } & \multicolumn{4}{|c|}{ Stoichiometric } & \multicolumn{4}{|c|}{ Rich } \\
\hline & \multicolumn{2}{|c|}{$\begin{array}{l}\text { Without } \\
\text { Catalyst }\end{array}$} & \multicolumn{2}{|c|}{$\begin{array}{c}\text { With } \\
\text { Catalyst }\end{array}$} & \multicolumn{2}{|c|}{$\begin{array}{l}\text { Without } \\
\text { Catalyst }\end{array}$} & \multicolumn{2}{|c|}{$\begin{array}{c}\text { With } \\
\text { Catalyst }\end{array}$} & \multicolumn{2}{|c|}{$\begin{array}{l}\text { Without } \\
\text { Catalyst }\end{array}$} & \multicolumn{2}{|c|}{$\begin{array}{c}\text { With } \\
\text { Catalyst }\end{array}$} \\
\hline & $\begin{array}{c}\text { FTP } \\
\mathrm{mg} / \mathrm{mi}\end{array}$ & $\begin{array}{c}\text { NMOG } \\
\text { wt \% }\end{array}$ & $\begin{array}{c}\text { FTP } \\
\mathrm{mg} / \mathrm{mi}\end{array}$ & $\begin{array}{c}\text { NMOG } \\
\text { wt \% }\end{array}$ & $\begin{array}{c}\mathrm{FTP} \\
\mathrm{mg} / \mathrm{mi}\end{array}$ & $\begin{array}{c}\text { NMOG } \\
\text { wt \% }\end{array}$ & $\begin{array}{c}\text { FTP } \\
\mathrm{mg} / \mathrm{mi}\end{array}$ & $\begin{array}{c}\text { NMOG } \\
\text { wt \% }\end{array}$ & $\begin{array}{c}\text { FTP } \\
\mathrm{mg} / \mathrm{mi}\end{array}$ & $\begin{array}{l}\text { NMOG } \\
\text { wt \% }\end{array}$ & $\begin{array}{c}\text { FTP } \\
\mathrm{mg} / \mathrm{mi}\end{array}$ & $\begin{array}{l}\text { NMOG } \\
\text { wt \% }\end{array}$ \\
\hline CYCLOPENTADIENE & 0.1 & $<0.1$ & ND & ND & 0.1 & $<0.1$ & ND & ND & 0.1 & $<0.1$ & ND & ND \\
\hline 2.2-DIMETHYLBUTANE & 0.1 & $<0.1$ & 20.1 & 0.1 & 0.2 & 20.1 & $=2.1$ & $<0.1$ & 0.4 & $3<01$. & 0.6 & .0 .1 \\
\hline CYCLOPENTENE & 0.2 & $<0.1$ & ND & ND & 0.1 & $<0.1$ & $<0.1$ & $<0.1$ & 0.2 & $<0.1$ & 0.1 & $<0.1$ \\
\hline 4-NETHYL-1 PENTENE & 0.1 & .80 .1 & $1 . \mathrm{ND}$ & . NO & 0.1 & $=2.1$ & 20.1 & .20 .1 & ND & NO & 0.1 & 201 \\
\hline 3-METHYL-1-PENTENE & $<0.1$ & $<0.1$ & 0.1 & 0.1 & $<0.1$ & $<0.1$ & $<0.1$ & $<0.1$ & $<0.1$ & $<0.1$ & 0.1 & $<0.1$ \\
\hline CYCLOPENTANE & ND & No & ND & No & $\mathrm{ND}$ & ND. & ND. & ND. & ND & ND & ND. & .0 .10 \\
\hline 2,3-DIMETHYLBUTANE & 0.3 & 0.1 & 0.1 & 0.1 & 0.2 & $<0.1$ & $<0.1$ & 0.1 & 0.5 & $<0.1$ & 0.7 & 0.2 \\
\hline MTBE & ND & ND & . NG. & $\mathrm{ND}$ & No & ND & ND & ND & ND & ND & $\mathrm{ND}$ & ND \\
\hline 2,3-DIMETHYL-1-BUTENE & ND & ND & ND & ND & ND & ND & ND & ND & ND & ND & ND & ND \\
\hline 4-METHYL-CIS-2-PENTENE & $=$ ND & $.2 \mathrm{NB}$ & NB. & $1.2 \mathrm{ND}$ & ND & $1 . \mathrm{ND}$ & NB & ND & No & ND & ND. & NB \\
\hline 2-METHYLPENTANE & 0.6 & 0.1 & 0.1 & 0.2 & 0.6 & 0.1 & 0.1 & 0.2 & 1.1 & 0.1 & 1.5 & 0.3 \\
\hline 4-METHYL-TRANS-2PENTENE & No & ND & ND & NO & ND & $1 . \mathrm{NB}$ & NB & NO & No & ND. & ND & ND. \\
\hline 3-METHYLPENTANE & 0.6 & 0.1 & 0.1 & 0.1 & 0.4 & 0.1 & 0.1 & 0.2 & 0.5 & $<0.1$ & 0.9 & 0.2 \\
\hline 2. METHYL - I-PENTENE & 200.1 & -2.1 & NO & . ND & NB. & $1 . \mathrm{ND}$ & NB & ND & ND &.$=\mathrm{NB}$. & 0.1 &.$<01$ \\
\hline 1.HEXENE & 0.2 & $<0.1$ & $<0.1$ & 0.1 & 0.1 & $<0.1$ & 0.1 & 0.2 & 0.3 & $<0.1$ & 0.1 & $<0.1$ \\
\hline HEXANE & 0.3 & 01. & 0.1 & $0.1 \%$ & .0 .1 & $(20.1 \%$ & 20.1 & $<0.1$ & 0.3 & $<0.1$ & 0.5 & (.). 0.1 . \\
\hline TRANS-3-HEXENE & $<0.1$ & $<0.1$ & $<0.1$ & $<0.1$ & 0.1 & $<0.1$ & $<0.1$ & $<0.1$ & $<0.1$ & $<0.1$ & 0.1 & $<0.1$ \\
\hline CIS 3.HEXENE & 0.1 & 80.1 & 20.1 & $<0.1$ & 0.1 & 20.1 & 20.1 & $<0.1$ & 0.1 & 80.1 & 0.1 & 20.1 \\
\hline TRANS-2-HEXENE & ND & ND & ND & ND & ND & ND & ND & ND & ND & ND & ND & ND \\
\hline 3.METHYL-TRANS 2-PENTENE &.$<0.1$ & $<0.1$ & ND & $.2 \mathrm{NO}$ & 0.2 & .20 .1 & 50.1 & $<0.1$ & 0.1 & 20.1 & 0.1 & $(20.1$. \\
\hline 2-METHYL-2-PENTENE & $<0.1$ & $<0.1$ & ND & ND & 0.1 & $<0.1$ & $<0.1$ & 0.1 & $<0.1$ & $<0.1$ & 0.1 & $<0.1$ \\
\hline 3METHYLCYCLOPENTENE & No & $\mathrm{ND}$ & $\mathrm{NO}$ & No & No & $.: \mathrm{NB}:$ & ND & NO & $\mathrm{NB}$ & NB & NB: & ND \\
\hline CIS-2-HEXENE & $<0.1$ & $<0.1$ & ND & ND & 0.1 & $<0.1$ & $<0.1$ & $<0.1$ & ND & ND & 0.1 & $<0.1$ \\
\hline ETBE & ND. & ND & ND & ND. & No & ND. & ND & ND & NB & NB & NB & ND \\
\hline 3-METHYL-CIS-2-PENTENE & 0.1 & $<0.1$ & $<0.1$ & 0.1 & 0.1 & $<0.1$ & $<0.1$ & $<0.1$ & 0.1 & $<0.1$ & 0.1 & $<0.1$ \\
\hline 22DIMETHYLPENIANE & 0.6 & 0.1 & 0.1 & $.0 .1 \%$ & 0.3 & 0.1 & $<0.1$ & 20.1 & 1,3 & 0.1 & 0.3 & 0.1 \\
\hline METHYLCYCLOPENTANE & ND & ND & $<0.1$ & 0.1 & 0.1 & $<0.1$ & ND & ND & ND & ND & ND & ND \\
\hline 2,4-DIMETHYLPENTANE & 0.2 & $<0.11$ & 0.1 & $0.1: 1$ & 0.2. & $<0.1$ & 0.1 & 0.1 & 0.4 & .20 .1$. & 0.4 & 0.11 \\
\hline 2,3,3-TRIMETHYL-1-BUTENE & ND & ND & ND & ND & ND & ND & ND & ND & ND & ND & ND & ND \\
\hline 2,23:TBIMETHVI BUITANE & $=0.81 .9$ & $=0.1 .21$ & 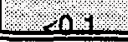 & $=.20,1, \ldots$ & $=. .60 .1$ & $=2011$ & 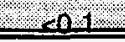 & -0.111 & 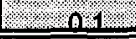 & $=\ln 1$ I I & $=0.1$ & $=-0.1$ \\
\hline
\end{tabular}


TABLE J-1 (CONT'D). SUMMARY OF SPECIATED EXHAUST EMISSIONS FROM CNG

\begin{tabular}{|c|c|c|c|c|c|c|c|c|c|c|c|c|}
\hline \multirow[b]{4}{*}{ Compound } & \multicolumn{12}{|c|}{ Operating Conditions } \\
\hline & \multicolumn{4}{|c|}{ Lean } & \multicolumn{4}{|c|}{ Stoichlometric } & \multicolumn{4}{|c|}{ Rich } \\
\hline & \multicolumn{2}{|c|}{$\begin{array}{l}\text { Without } \\
\text { Catalyst }\end{array}$} & \multicolumn{2}{|c|}{$\begin{array}{c}\text { With } \\
\text { Catalyst }\end{array}$} & \multicolumn{2}{|c|}{$\begin{array}{l}\text { Without } \\
\text { Catalyst }\end{array}$} & \multicolumn{2}{|c|}{$\begin{array}{c}\text { With } \\
\text { Catalyst }\end{array}$} & \multicolumn{2}{|c|}{$\begin{array}{l}\text { Without } \\
\text { Catalyst }\end{array}$} & \multicolumn{2}{|c|}{$\begin{array}{c}\text { With } \\
\text { Catalyst }\end{array}$} \\
\hline & $\begin{array}{c}\text { FTP } \\
\mathrm{mg} / \mathrm{mi}\end{array}$ & $\begin{array}{c}\text { NMOG } \\
\text { wt \% }\end{array}$ & $\begin{array}{c}\mathrm{FTP} \\
\mathrm{mg} / \mathrm{mi}\end{array}$ & $\begin{array}{c}\text { NMOG } \\
\text { wt \% }\end{array}$ & $\begin{array}{c}\mathrm{FTP} \\
\mathrm{mg} / \mathrm{mi}\end{array}$ & $\begin{array}{c}\text { NMOG } \\
\text { wt } \%\end{array}$ & $\begin{array}{c}\text { FTP } \\
\mathrm{mg} / \mathrm{mi}\end{array}$ & $\begin{array}{c}\text { NMOG } \\
\text { wt \% }\end{array}$ & $\begin{array}{c}\text { FTP } \\
\mathrm{mg} / \mathrm{mi}\end{array}$ & $\begin{array}{c}\text { NMOG } \\
\text { wt \% }\end{array}$ & $\begin{array}{c}\text { FTP } \\
\mathrm{mg} / \mathrm{ml}\end{array}$ & $\begin{array}{c}\text { NMOG } \\
\text { wt \% }\end{array}$ \\
\hline 3,4-DIMETHYL-1-PENTENE & ND & ND & ND & ND & ND & ND & ND & ND & ND & ND & ND & ND \\
\hline 1.METHYLCYCLOPENTENE & ND &. $\mathrm{ND}$ & ND & ND & ND & NO & ND & No & 01 & 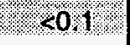 & 0.1 & -201 \\
\hline BENZENE & 0.5 & 0.1 & 0.1 & 0.2 & 0.5 & 0.1 & 0.2 & 0.5 & 0.8 & $<0.1$ & 1.0 & 0.2 \\
\hline 3-METHYL-1-HEXENE & NB & ND: & ND. & 6. $\mathrm{ND} !$ & ND & ND. & ND & ND & ND. & . $\mathrm{NO}$ & $\mathrm{ND}$ & ND. \\
\hline 3,3-DIMETHYLPENTANE & 0.1 & $<0.1$ & ND & ND & ND & ND & ND & ND & ND & ND & $<0.1$ & $<0.1$ \\
\hline CYCLOHEXANE & 20.1 & 20.1. & 20.1 & $<0.1$ & 20.1 & $<0.1$ & $<0.1$ & .20 .1 & $<0.1$ & $2.8 .1 \%$ & 0.1 & 201 \\
\hline 2-METHYLHEXANE & ND & ND & ND & ND & ND & ND & ND & ND & ND & ND. & ND & ND \\
\hline 23-DIMETHYVLPENTANE & 02 & 0.11 & $1.0 .1 \%$ & 0.11 & 0.3 & 0.1 & 0.1 & 0.1 & 0.5 & .20 .1 & 0.8 & 0.2 \\
\hline 1,1-DIMETHYLCYCLOPENTANE & ND & ND & ND & ND & ND & ND & ND & ND & ND & ND & ND & ND \\
\hline CYCLOHEXENE & ND & ND. & $\mathrm{ND}$ & ND & ND & ND. & $=\mathrm{NB}$ & NB. & NB & (1.: NO & ND. & NO. \\
\hline 3-METHYLHEXANE & 0.1 & $<0.1$ & 0.1 & 0.1 & 0.1 & $<0.1$ & $<0.1$ & $<0.1$ & 0.2 & $<0.1$ & 0.2 & $<0.1$ \\
\hline CIS.1,3-DIMETHYLCYCLOPENTANE & $<0$. & 80.1 & ND & . NB & NB & NB: & $=2.1$. & .20 .1 & ND & ND & $\mathrm{ND}$ & No \\
\hline 3-ETHYLPENTANE & ND & ND & ND & ND & ND & ND & ND & ND & ND & ND & ND & ND \\
\hline TAANS 1,2 DIMETHYL CYCLOPEN & ND & ND & ND & $2 . \mathrm{ND}$ & ND & NB & NO & NO & ND & ND & ND & No \\
\hline TRANS-1,3-DIMETHYLCYCLOPENTANE & ND & ND & ND & ND & ND & ND & ND & ND & ND & ND & ND & ND \\
\hline HEPTENE & ND & ND & ND & $\mathrm{ND}$ & ND. & ND & ND & NO & NO & ND & No. & ND. \\
\hline 2,2,4-TRIMETHYLPENTANE & 0.3 & 0.1 & 0.2 & 0.2 & 0.3 & 0.1 & 0.1 & 0.3 & 0.6 & $<0.1$ & 0.9 & 0.2 \\
\hline 2.METHYL 1 HEXENE & No & ND & No & ND & ND & ND & ND & ND & ND & $\mathrm{NB}$ & $\mathrm{ND}$ & ND: \\
\hline TRANS-3-HEPTENE & ND & ND & ND & ND & ND & ND & ND & ND & ND & ND & ND & ND \\
\hline HEPTANE & 0.2 & $2011:$ & $=0.1$ & 0.2 & 0.1 & 20.1 & $<0.1$ & 0.4 & 0.1 & 20.1 & $<0.1$. & 201 \\
\hline 2-METHYL-2-HEXENE & ND & ND & ND & ND & ND & ND & ND. & ND & ND: & ND & ND & ND \\
\hline 3 METHYLTRANS 3 HEXENE & ND & No & No &. $\mathrm{ND}$. & ND & . ND & $\mathrm{NB}$ & NO & NB & $\mathrm{NB}$ & NO & $\mathrm{NO}$ \\
\hline TRANS-2-HEPTENE & ND & ND & ND & ND & ND & ND & ND & ND: & ND & ND & ND & ND \\
\hline 3ETHYLCIS-2-PENTENE & ND & ND & . ND & ND. & ND & NO & ND & NO & NO & $\mathrm{NB}$ & $\mathrm{ND}$ & NB: \\
\hline 2,4,4-TRIMETHYL-1-PENTENE & ND & ND & ND & ND & $<0.1$ & $<0.1$ & ND & ND & ND & ND & ND & ND \\
\hline 2,2,4-TRIMETHYL-1 PENTENE & NB & $\mathrm{NB}=$ & NO & NB. & ND & NB & No & ND & ND & ND & ND & ND: \\
\hline 2,3-DIMETHYL-2-PENTENE & ND & ND & ND & ND & ND & ND & ND & ND & $N D$ & ND & ND & ND \\
\hline GS.2.HEPTENE & No. & No & $<0.1$ & $20.1 \%$ & 0.1 & $<0.1$ & 20.1 & 20.1 & NB & $\mathrm{NB}$ & $<0.1$ & $<0.1$ \\
\hline METHYLCYCLOHEXANE & ND & ND & ND & ND & ND & ND & ND & ND & ND & ND & ND & ND \\
\hline CIS 1, 2.DIMETHYL GYCLOPENI & ND & No & $=\mathrm{ND}$ & ND & ND & ND. & $\mathrm{NO}$ & $\mathrm{ND}$ & $\mathrm{NB}$ & $\mathrm{ND}$ & No & No \\
\hline
\end{tabular}


TABLE J-1 (CONT'D). SUMMARY OF SPECIATED EXHAUST EMISSIONS FROM CNG

\begin{tabular}{|c|c|c|c|c|c|c|c|c|c|c|c|c|}
\hline \multirow[b]{4}{*}{ Compound } & \multicolumn{12}{|c|}{ Operating Conditlons } \\
\hline & \multicolumn{4}{|c|}{ Lean } & \multicolumn{4}{|c|}{ Stoichiometric } & \multicolumn{4}{|c|}{ Rich } \\
\hline & \multicolumn{2}{|c|}{$\begin{array}{l}\text { Without } \\
\text { Catalyst }\end{array}$} & \multicolumn{2}{|c|}{$\begin{array}{c}\text { With } \\
\text { Catalyst } \\
\end{array}$} & \multicolumn{2}{|c|}{$\begin{array}{l}\text { Without } \\
\text { Catalyst }\end{array}$} & \multicolumn{2}{|c|}{$\begin{array}{c}\text { With } \\
\text { Catalyst }\end{array}$} & \multicolumn{2}{|c|}{$\begin{array}{l}\text { Without } \\
\text { Catalyst }\end{array}$} & \multicolumn{2}{|c|}{$\begin{array}{c}\text { With } \\
\text { Catalyst }\end{array}$} \\
\hline & $\begin{array}{c}\mathrm{FTP} \\
\mathrm{mg} / \mathrm{mi}\end{array}$ & $\begin{array}{c}\text { NMOG } \\
\text { wt \% } \\
\end{array}$ & $\begin{array}{c}\text { FTP } \\
\mathrm{mg} / \mathrm{mi}\end{array}$ & $\begin{array}{c}\text { NMOG } \\
\text { wt \% }\end{array}$ & $\begin{array}{l}\text { FTP } \\
\mathrm{mg} / \mathrm{mi}\end{array}$ & $\begin{array}{c}\text { NMOG } \\
\text { wt \% }\end{array}$ & $\begin{array}{c}\text { FTP } \\
\mathrm{mg} / \mathrm{mi} \\
\end{array}$ & $\begin{array}{c}\text { NMOG } \\
\text { wt \% } \\
\end{array}$ & $\begin{array}{l}\text { FTP } \\
\mathrm{mg} / \mathrm{mi}\end{array}$ & $\begin{array}{c}\text { NMOG } \\
w t \%\end{array}$ & $\begin{array}{c}\text { FrP } \\
\mathrm{mg} / \mathrm{mi}\end{array}$ & $\begin{array}{c}\text { NMOG } \\
\text { wt \% } \\
\end{array}$ \\
\hline 2,2-DIMETHYLHEXANE & 0.2 & $<0.1$ & ND & ND & 0.1 & $<0.1$ & $<0.1$ & $<0.1$ & 0.1 & $<0.1$ & 0.1 & $<0.1$ \\
\hline 1, 1,3-TRIMETHYLCYCLOPENTANE & NB & (1. ND. & $\mathrm{ND}$ & ND. & NB & $2.1 \mathrm{NO}$ & NO & No & NB & ND & No & NB \\
\hline 2,4,4-TRIMETHYL-2-PENTENE & ND & ND & ND & ND & ND & ND & ND & ND & ND & ND & ND & ND \\
\hline $2,2,3$ TRIMETHYLPENTANE & No & ND & ND. & ND $:$ & ND & NB & ND. & ND. & NB. & ND. & ND & ND: \\
\hline 2,5-DIMETHYLHEXANE & ND & ND & ND & ND & ND & ND & ND & ND & ND & ND & ND & ND \\
\hline ETHYLCYCLOPENTANE & No & . ND. & ND. & No & ND. & NO & NB: & ND. & NB: & No & ND & No \\
\hline 2,4-DIMETHYLHEXANE & $<0.1$ & $<0.1$ & ND & ND & 0.1 & $<0.1$ & $<0.1$ & 0.1 & 0.1 & $<0.1$ & 0.1 & $<0.1$ \\
\hline 1-TRANS-2-CIS-4-TRIMETHYLCYCLOPENTANE & No & (.) ND & No. & ND & ND. & ND. & ND & ND & ND. & NB & ND & ND \\
\hline 3,3-DIMETHYLHEXANE & 0.1 & $<0.1$ & ND & ND & ND & ND & ND & ND & ND & ND & ND & ND \\
\hline 1TRANS2-C1S-3-TRIMETHYLCYCLOPENTANE & \%. $\mathrm{ND}$ & 1. ND.: & 1. NB & ND. & ND & ND. & ND & NO & $.2 \mathrm{NO}$ & ND 1 & ND & ND \\
\hline 2,3,4-TRIMETHYLPENTANE & ND & ND & ND & ND & ND & ND & ND & ND & ND & ND & ND & ND \\
\hline 2,3,3-TRIMETHYLPENTANE & 20.1 & 20.1 & $=0.1$ & 201 & 20.1 & 20.1 & 01 & 0.1 & $0.1 \%$ & $=201$ & $0.1 \%$ & 20.1 \\
\hline TOLUENE & 0.4 & 0.1 & $<0.1$ & 0.1 & 0.5 & 0.1 & 0.4 & 0.9 & 0.9 & 0.1 & 0.3 & 0.1 \\
\hline 2,3-DIMETHYLHEXANE & ND & ND & NO & ND & ND & No & ND & ND & $\mathrm{ND}$ & ND & ND & NB. \\
\hline 1,1,2-TRIMETHYLCYCLOPENTANE & ND & ND & ND & ND & ND & ND & ND & ND & ND & ND & ND & ND \\
\hline 2-METHYLHEPTANE & ND. & ND: & ND & NB & ND. & $\mathrm{ND}$. & 0.1 & 0.1. & 20.1 & $<0.1$ & 02 & 60.1. \\
\hline 3,4-DIMETHYLHEXANE & ND & ND & ND & ND & ND & ND & ND & ND & ND & ND & ND & ND \\
\hline 2, 2, 4, 4,TETRAMETHYLPENTANE: & No & ND? & NO & ND. & NO & NB & No & ND. & $.1 . \mathrm{NB}$ & $\mathrm{ND}$ & ND. & $\mathrm{ND}$ \\
\hline 4-METHYLHEPTANE & ND & ND & ND & ND & $<0.1$ & $<0.1$ & ND & ND & ND & ND & ND & ND \\
\hline 2METHYL-3-ETHYLPENTANE & ND & ND & ND. & ND & ND & - ND & $\mathrm{NB}$ & ND & ND. & ND & $=\mathrm{ND}$ & ND \\
\hline 2,6-DIMETHYLHEPTANE & ND & ND & ND & ND & ND & ND & ND & ND & ND & ND & ND & ND \\
\hline 3 METHYLHEPTANE : & $\%=\mathrm{sol}$ & .01 & $1 . \mathrm{NO}$ & ND. 1 & 80.1 & 80.1 & 0.1 & 0.1 .1 & $<0.1$ & $20.1 \%$ & 01. & <0. \\
\hline 1-CIS,2-TRANS,3-TRIMETHYLCYCLOPENTANE & ND & ND & ND & ND & ND & ND & ND & ND & ND & ND & ND & ND \\
\hline CIS-1,3 DIMETHYLCYCLOHEXANE & 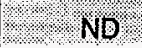 & . $\mathrm{ND}$ & ND. & ND & NB & NO & ND. & ND & ND. & ND & $=\mathrm{ND}$ & No \\
\hline TRANS-1,4-DIMETHYLCYCLOHEXANE & 0.1 & $<0.1$ & ND & ND & ND & ND & $<0.1$ & $<0.1$ & ND & ND & $<0.1$ & $<0.1$ \\
\hline 3ETHYLHEXANE & ND. & ND & No. & ND & ND & ND & ND. & ND. & No. & ND & No & No \\
\hline 2,2,5-TRIMETHYLHEXANE & ND & ND & ND & ND & ND & ND & ND & ND & ND & ND. & ND & ND \\
\hline CIS-1 METHYL-3ETHYLCYCLOPENTANE & ND & ND. & ND & ND 1 & NO & ND & ND & ND & No. & No & ND & No \\
\hline 1,1-DIMETHYLCYCLOHEXANE & ND & ND & ND & ND & ND & ND & ND & ND & ND & ND & ND & ND \\
\hline GRANS.1.METHYL.2 ETHYLCYCLOPENTANE & $2 . \mathrm{NB}$ & ND & $\mathrm{ND}$ & ND. & NO & NO & $\mathrm{NB}$ & NO & ND & ND. & ND. & ND. \\
\hline
\end{tabular}


TABLE J-1 (CONT'D). SUMMARY OF SPECIATED EXHAUST EMISSIONS FROM CNG

\begin{tabular}{|c|c|c|c|c|c|c|c|c|c|c|c|c|}
\hline \multirow[b]{4}{*}{ Compound } & \multicolumn{12}{|c|}{ Operating Conditions } \\
\hline & \multicolumn{4}{|c|}{ Lean } & \multicolumn{4}{|c|}{ Stoichiometric } & \multicolumn{4}{|c|}{ Rich } \\
\hline & \multicolumn{2}{|c|}{$\begin{array}{l}\text { Without } \\
\text { Catalyst }\end{array}$} & \multicolumn{2}{|c|}{$\begin{array}{c}\text { With } \\
\text { Catalyst }\end{array}$} & \multicolumn{2}{|c|}{$\begin{array}{l}\text { Without } \\
\text { Catalyst }\end{array}$} & \multicolumn{2}{|c|}{$\begin{array}{c}\text { With } \\
\text { Catalyst }\end{array}$} & \multicolumn{2}{|c|}{$\begin{array}{l}\text { Without } \\
\text { Catalyst }\end{array}$} & \multicolumn{2}{|c|}{$\begin{array}{c}\text { With } \\
\text { Catalyst }\end{array}$} \\
\hline & $\begin{array}{c}\text { FTP } \\
\mathrm{mg} / \mathrm{mi}\end{array}$ & $\begin{array}{c}\text { NMOG } \\
\text { wt \% }\end{array}$ & $\begin{array}{c}\text { FTP } \\
\mathrm{mg} / \mathrm{mi}\end{array}$ & $\begin{array}{c}\text { NMOG } \\
\text { wt \% }\end{array}$ & $\begin{array}{c}\text { FTP } \\
\mathrm{mg} / \mathrm{mi}\end{array}$ & $\begin{array}{c}\text { NMOG } \\
\text { wt \% }\end{array}$ & $\begin{array}{c}\text { FTP } \\
\mathrm{mg} / \mathrm{mi}\end{array}$ & $\begin{array}{c}\text { NMOG } \\
\text { wt } \%\end{array}$ & $\begin{array}{c}\text { FTP } \\
\mathrm{mg} / \mathrm{mi}\end{array}$ & $\begin{array}{c}\text { NMOG } \\
\text { wt \% }\end{array}$ & $\begin{array}{c}\text { FTP } \\
\mathrm{mg} / \mathrm{mi}\end{array}$ & $\begin{array}{c}\text { NMOG } \\
\text { wt \% }\end{array}$ \\
\hline 1-METHYL-1-ETHYL-CYCLOPENTANE & ND & ND & $\overline{N D}$ & ND & ND & ND & ND & ND & ND & ND & ND & ND \\
\hline 2,4,4.TRIMETHYLHEXANE & NO & . ND. & ND & NB & No & $\mathrm{NB}:$ & ND & NB. & NO. & $.2 .1 \mathrm{NB}$ & No & ND \\
\hline 2,2,4-TRIMETHYLHEXANE & ND & ND & ND & ND & ND & ND & ND & ND & ND & ND & ND & ND \\
\hline TRANS-1,2 DIMETHYLCYCLOHEXA & ND & $=\mathrm{NB}$ & ND & ND & ND. & NB & No & NO & No & ND & ND & ND \\
\hline 1-OCTENE & ND & ND & ND & ND & ND & ND & ND & ND & ND & ND & ND & ND \\
\hline TRANS-4. OCTENE & ND & .1 .20 & $1.2 \mathrm{ND}$ & : NO & ND & ND & (1. NB & ND: & ND. & ND & No & ND \\
\hline OCTANE & ND & ND & ND & ND & ND & ND & $<0.1$ & 0.1 & ND & ND & 0.1 & $<0.1$ \\
\hline TRANS 2. OCTENE & $1.2 . \mathrm{NO}$ & ND & No. & ND & ND & ND & NO & NB & ND & No & ND & ND \\
\hline TRANS-1,3-DIMETHYLCYCLOHEXAN & ND & ND & ND & ND & ND & ND & ND & ND & ND & ND & ND & ND \\
\hline CIS 1,4 - DIMETHYLCYCLOHEXANE & ND & : No & ND & ND. & $\mathrm{NB}$ & NO & ND & ND & ND & ND & ND & ND \\
\hline CIS-2-OCTENE & ND & ND & ND & ND & ND & ND & ND & ND & ND & ND & ND & ND \\
\hline 2.3.5-TRIMETHYLHEXANE & NB. & ND & $\mathrm{NO}$ & NO. & $\mathrm{ND}$ &. $\mathrm{ND}$ & ND & NB & ND. & NB: & ND. & ND \\
\hline CIS-1-METHYL-2-ETHYLCYCLOPENTANE & ND & ND & ND & ND & ND & ND & ND & ND & ND & ND & ND. & ND \\
\hline 2.METHYL-2-ETHYLHEPTANE ? ? & ND. & ND. & ND & ND & ND & . NB. & NO & ND & ND. & ND & ND & NB \\
\hline 2,4-DIMETHYLHEPTANE & 0.2 & $<0.1$ & 0.4 & 0.7 & 0.5 & 0.1 & 0.6 & 1.4 & 0.3 & $<0.1$ & 0.5 & 0.1 \\
\hline 4.4-DIMETHYLLEPTANE & ND & No & $\mathrm{ND}$ & NB & ND. & 2. ND. & $\mathrm{ND}$ & ND. & ND & NB. & No & No \\
\hline CIS-1,2-DIMETHYLCYCLOHEXANE & ND & ND & ND & ND & ND & ND & ND & ND & ND & ND & ND & ND \\
\hline ETHYLCYCLOHEXANE & ND & ND & ND. & ND & ND & No. & NB & No & No & $\mathrm{ND}$ & ND & NO \\
\hline PROPYLCYCLOHEXANE & ND & ND & ND & ND & ND & ND & ND & ND & ND & ND & ND & ND \\
\hline 2.METHYL-4.ETHYLHEXANE. & ND & (. $\mathrm{ND}$ & $\mathrm{NB}$ & Nb. & NO & $=2$ No & NB & ND & ND. & ND: & NB & ND \\
\hline 2,6-DIMETHYLHEPTANE & ND & ND & ND & ND & ND & ND & ND & ND & ND & ND & ND & ND \\
\hline 1,1,3-TRIMETHYLCYCLOHEXANE & $\mathrm{ND}$ & ND & $\mathrm{ND}$ & . ND & ND & $\mathrm{ND}$ & No & ND. & (. NO & . NB: & ND & ND \\
\hline 2,5-DIMETHYLHEPTANE & ND & ND & ND & ND & ND & ND & ND & ND & ND & ND & ND & ND \\
\hline 3,3.DIMETHYLHEPTANE & 1.20 & ND: & 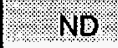 & . ND. & ND & $\mathrm{NB}$ & ND & NB & ND & ND & NB. & ND \\
\hline 3,5-DIMETHYLHEPTANE & ND & ND & ND & ND & ND & ND & ND & ND & ND & ND & ND & ND \\
\hline ETHYLBENZENE: & 0.1 & 201 & $0.1 \%$ & 0.1 & 0.1 & 2.20 .1$. & 20.1 & .20 .1 & 0.1 & .20 .1 & 0.1 & $<0.1$ \\
\hline 2,3-DIMETHYLHEPTANE & ND & ND & ND & ND & ND & ND & ND & ND & ND & ND & ND & ND \\
\hline m- $\&$ p-XYLENE & 0.0 .3 & 0.1 & 02 & 0.2 & 0.1 & $<0.1$ & 0.1 & 0.1 & 1.0 .3 & $<0.1$ & 02 & 0.1 \\
\hline 4-METHYLOCTANE & ND & ND & ND & ND & ND & ND & ND & ND & ND & ND & ND & ND \\
\hline E.METHYLOCTANE & ND & ND. & $\mathrm{NB}$ & ND. & 1 NE & NO & No & ND & 1.2 .1 .20 & ND & ND & ND \\
\hline
\end{tabular}


TABLE J-1 (CONT'D). SUMMARY OF SPECIATED EXHAUST EMISSIONS FROM CNG

\begin{tabular}{|c|c|c|c|c|c|c|c|c|c|c|c|c|}
\hline \multirow[b]{4}{*}{ Compound } & \multicolumn{12}{|c|}{ Operating Conditions } \\
\hline & \multicolumn{4}{|c|}{ Lean } & \multicolumn{4}{|c|}{ Stoichiometric } & \multicolumn{4}{|c|}{ Rich } \\
\hline & \multicolumn{2}{|c|}{$\begin{array}{l}\text { Without } \\
\text { Catalyst }\end{array}$} & \multicolumn{2}{|c|}{$\begin{array}{c}\text { With } \\
\text { Catalyst }\end{array}$} & \multicolumn{2}{|c|}{$\begin{array}{l}\text { Without } \\
\text { Catalyst }\end{array}$} & \multicolumn{2}{|c|}{$\begin{array}{c}\text { With } \\
\text { Catalyst }\end{array}$} & \multicolumn{2}{|c|}{$\begin{array}{l}\text { Without } \\
\text { Catalyst }\end{array}$} & \multicolumn{2}{|c|}{$\begin{array}{c}\text { With } \\
\text { Catalyst }\end{array}$} \\
\hline & $\begin{array}{c}\text { FTP } \\
\mathrm{mg} / \mathrm{mi}\end{array}$ & $\begin{array}{c}\text { NMOG } \\
\text { wt } \% \\
\end{array}$ & $\begin{array}{c}\text { FTP } \\
\mathrm{mg} / \mathrm{mi}\end{array}$ & $\begin{array}{c}\text { NMOG } \\
\text { wt \% }\end{array}$ & $\begin{array}{c}\text { FTP } \\
\mathrm{mg} / \mathrm{mi}\end{array}$ & $\begin{array}{c}\text { NMOG } \\
\text { wt \% }\end{array}$ & $\begin{array}{c}\text { FTP } \\
\mathrm{mg} / \mathrm{mi}\end{array}$ & $\begin{array}{c}\text { NMOG } \\
\text { wt } \% \\
\end{array}$ & $\begin{array}{c}\text { FTP } \\
\mathrm{mg} / \mathrm{mi}\end{array}$ & $\begin{array}{c}\text { NMOG } \\
\text { wt \% }\end{array}$ & $\begin{array}{c}\text { FTP } \\
\mathrm{mg} / \mathrm{mi}\end{array}$ & $\begin{array}{l}\text { NMOG } \\
\text { wt \% }\end{array}$ \\
\hline 3-METHYLOCTANE & ND & ND & ND & NO & $<0.1$ & $<0.1$ & ND & ND & ND & ND & ND & ND \\
\hline STYRENE & ND & 1. NB & $\mathrm{NB}$ & No & ND & $\mathrm{NB}$ & No $\mathrm{NB}$ & $\mathrm{NB}$ & ND & $1 . \mathrm{ND}$ & ND & ND \\
\hline O-XYLENE & 0.2 & $<0.1$ & 0.1 & 0.2 & 0.1 & $<0.1$ & $<0.1$ & $<0.1$ & 0.1 & $<0.1$ & 0.1 & $<0.1$ \\
\hline 2,4,6 TRIMETHYLHEXANE & ND. & ND & $\mathrm{NB}$ & ND. & ND & ND & ND. & NO & ND & ND & NLS: & ND \\
\hline 1-NONENE & ND & ND & ND & ND & ND & ND & ND & ND & ND & ND & ND. & ND \\
\hline NONANE & 20.1. & .80 .1 & ND & (.) $\mathrm{ND}:$ & ND & NB & NB. & 1. NB & ND & ND & ND & ND \\
\hline ISOPROPYLBENZENE (CUMENE) & ND & ND & ND & ND & ND & ND & $<0.1$ & $<0.1$ & ND & ND & ND & ND \\
\hline 2,2-DIMETHYLOCTANE & No. & ND & NO & ND & ND & ND. & ND & ND & ND & NB & $\mathrm{ND}$ & ND \\
\hline 2,4-DIMETHYLOCTANE & 0.3 & 0.1 & 0.1 & 0.1 & 0.2 & $<0.1$ & 0.1 & 0.2 & $<0.1$ & $<0.1$ & 0.2 & $<0.1$ \\
\hline D-PROPYLBENZENE & ND & ND & ND & ND & No & ND & NO & ND. & ND & NO & ND & ND: \\
\hline 1-METHYL-3-ETHYLBENZENE & 0.1 & $<0.1$ & 0.1 & 0.2 & $<0.1$ & $<0.1$ & 0.1 & 0.2 & 0.1 & $<0.1$ & $<0.1$ & $<0.1$ \\
\hline Н METHYL-4-ETHYLBENZENE & $<0.1$ & 20.1 & $\mathrm{ND}$ & ND & ND & $=\mathrm{NB}$ & 80.1 & $<0.1$ & NB. & ND. & No & ND. \\
\hline 1,3,5-TRIMETHYLBENZENE & 0.1 & $<0.1$ & ND & ND & ND & ND & $<0.1$ & $<0.1$ & ND & ND & ND & ND \\
\hline 1.METHYL-2-ETHYLBENZENE & 0.4 & 0.1. & ND &. $\mathrm{ND}$ & ND & ND. & . .10 & NB & 80.1 & $<0.1$ & ND & ND \\
\hline 1,2,4-TRIMETHYLBENZENE & 0.1 & $<0.1$ & 0.1 & 0.1 & 0.2 & $<0.1$ & $<0.1$ & 0.1 & 0.1 & $<0.1$ & $<0.1$ & $<0.1$ \\
\hline DECANE & 0.1 & -80.1 & $20.1 \%$ & .0 .1 & 0.1 & 20.1 & 0.1 & 0.2 & 0.1 & $\times 0.1$ & 0.1 & -801 \\
\hline ISOBUTYLBENZENE & 0.1 & $<0.1$ & $<0.1$ & 0.1 & 0.1 & $<0.1$ & 0.1 & 0.2 & 0.1 & $<0.1$ & 0.1 & $<0.1$ \\
\hline METHYLPROPYLBENZENE & NO & No & $1 . \mathrm{NO}$ & ND & No & ND & NO & ND: & No & No & ND. & No. \\
\hline S-BUTYLBENZENE & ND & ND & ND & ND & ND & ND & ND & ND & ND & ND & ND & ND \\
\hline 1.METHYL 3 ISOPROPYLEENZEN & $<0.1$ & .1 .20 .1 & ND & No & 80.1 & $<0.1$ & 80.1 & 20.1 & 50.1 & 20.1 & NB & No \\
\hline 1,2,3-TRIMETHYLBENZENE & ND & ND & ND & ND & ND & ND & ND & ND & ND & ND & ND & ND \\
\hline TMETHYL 4 ISOPROPYLBENZENE & ND & ND: & 1. ND & $\mathrm{ND}$ & 0.1 & 1.80 .1 & $\mathrm{NO}$ & $\mathrm{ND}$ & 20.1 & <0 & ND. & ND \\
\hline IND AN & ND & ND & ND & ND & ND & ND & ND & ND & ND & ND & ND & ND \\
\hline 1. NETHYL 2. ISOPBOPYLBENZENE & ND & ND & ND. & ND & 0.1 & $<0.1$ & $\mathrm{ND}$ & NB: & 0.1 & 20.1 & ND & NO \\
\hline 1,3-DIETHYLBENZENE & ND & ND & ND & ND & ND & ND & ND & ND & ND & ND & ND & ND \\
\hline 1,4-DIETHYLBENZENE: & $(80.1$ & 20.1 : & ND & ND & 0.1 & -0.1 & $=\mathrm{NB}$ & ND & NB & $\mathrm{ND}$ & $<01$. & 20.1 \\
\hline 1-METHYL-3-N-PROPYLBENZENE & ND & ND & ND & ND & ND & ND & $<0.1$ & $<0.1$ & ND & ND & ND & ND \\
\hline 1 METHYL-4-N-PROPYLBENZENE & 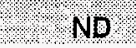 & ND & NO & ND & $<0.1$ & 20.1. & $\mathrm{NB}$ & NB & ND & ND & ND & ND \\
\hline 1,2 DIETHYLBENZENE & ND & ND & ND & ND & ND & ND & ND & ND & ND & ND & ND & ND \\
\hline 1.METHYL-2N-PROPYLBENZENE & No. & ND & $\mathrm{NB}$ & No. & ND & ND & ND. & ND & $\mathrm{NB}$ & ND & $2 . \mathrm{ND}$ & ND \\
\hline
\end{tabular}


TABLE J-1 (CONT'D). SUMMARY OF SPECIATED EXHAUST EMISSIONS FROM CNG

\begin{tabular}{|c|c|c|c|c|c|c|c|c|c|c|c|c|}
\hline \multirow[b]{4}{*}{ Compound } & \multicolumn{12}{|c|}{ Operating Conditions } \\
\hline & \multicolumn{4}{|c|}{ Lean } & \multicolumn{4}{|c|}{ Stoichlometric } & \multicolumn{4}{|c|}{ Rich } \\
\hline & \multicolumn{2}{|c|}{$\begin{array}{l}\text { Without } \\
\text { Catalyst }\end{array}$} & \multicolumn{2}{|c|}{$\begin{array}{c}\text { With } \\
\text { Catalyst }\end{array}$} & \multicolumn{2}{|c|}{$\begin{array}{l}\text { Without } \\
\text { Catalyst }\end{array}$} & \multicolumn{2}{|c|}{$\begin{array}{c}\text { With } \\
\text { Catalyst }\end{array}$} & \multicolumn{2}{|c|}{$\begin{array}{l}\text { Without } \\
\text { Catalyst }\end{array}$} & \multicolumn{2}{|c|}{$\begin{array}{c}\text { With } \\
\text { Catalyst } \\
\end{array}$} \\
\hline & $\begin{array}{c}\text { FTP } \\
\mathbf{m g} / \mathbf{m i}\end{array}$ & $\begin{array}{c}\text { NMOG } \\
\text { wt \% }\end{array}$ & $\begin{array}{c}\text { FTP } \\
\mathrm{mg} / \mathrm{mi}\end{array}$ & $\begin{array}{c}\text { NMOG } \\
\mathbf{w t} \% \\
\end{array}$ & $\begin{array}{c}\text { FTP } \\
\mathrm{mg} / \mathrm{mi}\end{array}$ & $\begin{array}{c}\text { NMOG } \\
\text { wt \% }\end{array}$ & $\begin{array}{c}\text { FTP } \\
\mathrm{mg} / \mathrm{mi}\end{array}$ & $\begin{array}{c}\text { NMOG } \\
\text { wt \% }\end{array}$ & $\begin{array}{c}\text { FTP } \\
\mathrm{mg} / \mathrm{mi}\end{array}$ & $\begin{array}{c}\text { NMOG } \\
\text { wt \% }\end{array}$ & $\begin{array}{c}\text { FTP } \\
\mathrm{mg} / \mathrm{mi}\end{array}$ & $\begin{array}{c}\text { NMOG } \\
\text { wt \% }\end{array}$ \\
\hline 1,4-DIMETHYL-2-ETHYLBENZENE & ND & ND & ND & ND & $<0.1$ & $<0.1$ & ND & ND & $<0.1$ & $<0.1$ & $<0.1$ & $<0.1$ \\
\hline 13-DIMETHYL-4-ETHYLBENZENE & ND. & NO & ND & ND. & NO & 1.2 .10 & No. & NB. & No & ND & ND & ND \\
\hline 1,2-DIMETHYL-4-ETHYLBENZENE & ND & ND & $<0.1$ & 0.1 & ND & ND & ND & ND & ND & ND & ND & ND \\
\hline 1.3-BIMETHYL-2-TTHYLBENZENE ? & 1.0 .0$. & ND & ND & ND & 20.1 & $2.80 .1 ?$ & ND & ND & ND & $\mathrm{NB}$ & ND & NO \\
\hline UND ECANE & $<0.1$ & $<0.1$ & $<0.1$ & $<0.1$ & 0.1 & $<0.1$ & 0.1 & 0.3 & $<0.1$ & $<0.1$ & $<0.1$ & $<0.1$ \\
\hline 1,2 DIMETHYL-3-ETHYLBENZENE: & ND. & $1.2 \mathrm{NB}$ & . ND & ND. & No & ND. & No. & ND & ND & $\mathrm{ND}$ & ND & ND \\
\hline 1,2,4,5-TETRAMETHYLBENZENE & 1.2 & 0.3 & 0.1 & 0.2 & ND & ND & ND & ND & $<0.1$ & $<0.1$ & ND & ND \\
\hline 2.METHYLBUTYLBENZENE (SEC AMYLBENZENE) : & . ND & ND & 1. ND. & . ND. & NE. & . $\mathrm{NO}$ & No: & ND. & ND & $\mathrm{ND}$ & ND & ND \\
\hline 3,4 DIMETHYLCUMENE & ND & ND & ND & ND & ND & ND & ND & ND & ND & ND & ND & ND \\
\hline $1,2,3,5-T E T R A M E T H Y L B E N Z E N E$ & $1 .: \mathrm{ND}$ &. $\mathrm{ND}$ & . .10 & $.1 \mathrm{NO}$ & NB & ND & ND & ND & ND & NB & ND & No \\
\hline TERT-1-BUT-2-METHYLBENZENE & ND & ND & ND & ND & $<0.1$ & $<0.1$ & ND & ND & ND & ND & ND & ND \\
\hline $1,2,3,4-T E T R A M E T H T Y$ BENZENE & $1.2 \mathrm{ND}:$ & . ND. & ND & ND & ND. & ND & 1. NG & $\mathrm{NB}$ & ND & ND & ND & ND: \\
\hline N-PENT-BENZENE & 0.1 & $<0.1$ & ND & ND & $<0.1$ & $<0.1$ & ND & ND & ND & ND & ND & ND \\
\hline TERT 1.BUT 3,5.DIMETHYLBENZENE & $\times 0.1$ & $<0.1$ & ND. & ND. & 01. & 2.20 .1 & 0.1 & 0.2 & ND & ND & ND & ND \\
\hline NAPHTHALENE & ND & ND & ND & ND & ND & ND & ND & ND & ND & ND & ND & ND \\
\hline DODECANE & 20.1 & $.0 .1 \div$ & .80 .1 &.$<0.1$. & 0.1 & $(.2 .80 .11$. & 0.1 .4 & 1.0. & .80 .1 & $.0 .1 \%$ & 20.1 & $<0.1$ \\
\hline FORMALDEHYDE & 92.3 & 20.3 & 1.2 & 2.0 & 70.4 & 13.0 & 0.9 & 2.0 & 137.0 & 7.6 & 1.5 & 0.3 \\
\hline ACETALDEHYDE & 4.2 & .0 .9 & 0.1 & 0.2 & 37. & 0.7 & 0.1 & 02 & 7.9 & 0.4. & 0.3 & 01 \\
\hline ACROLEIN & ND & ND & ND & ND & ND & ND & ND & ND & ND & ND & ND & ND \\
\hline ACETONE & .011 & $<0.1$ & 0.1. & 0.2 & $<0.1$ & .80 .1$. & 0.1 & 0.11 & 201 & 20.1 & No & NO \\
\hline PROPIONALDEHYDE & 0.1 & $<0.1$ & 0.1 & 0.2 & $<0.1$ & $<0.1$ & 0.1 & 0.1 & $<0.1$ & $<0.1$ & $<0.1$ & $<0.1$ \\
\hline CROTONALDEHYDE & 1.0 .0 .2 & $0.1 \%$ & \%. 0.3 . & 0.5 & 0.9 & 0.2 & 0.4 & 0.8 & 1.8 .8 & 0.1 & 0.3 & 0.1 \\
\hline ISOBUTYRALDEHYDE & $<0.1$ & $<0.1$ & ND & ND & ND & ND & ND & ND & ND & ND & ND & ND \\
\hline METHYL ETHYL KETONE & ?. ND & ND. & . NB. & ND & $\mathrm{NB}$ & ND. & ND & NB & ND & No & ND & ND \\
\hline BENZALDEHYDE & ND & ND & ND & ND & ND & ND & ND & ND & ND & ND & ND & ND \\
\hline HEXANALDEHYDE & . ND. & ND & ND & ND & $\mathrm{NB}$ & ND: & $\mathrm{NB}$ & No & ND & No & No & ND. \\
\hline SUMMATION OF NON-METHANE COMPOUNDS & 451.5 & 99.4 & 60.3 & 98.7 & 538.0 & 99.1 & 44.1 & 98.8 & 1805.7 & 99.8 & 443.5 & 99.7 \\
\hline
\end{tabular}




\section{APPENDIX K}

\section{AVERAGE SPECIATED EMISSIONS RESULTS FROM FTPS WITH REFORMULATED GASOLINE}


TABLE K-1. SUMMARY OF SPECIATED EXHAUST EMISSIONS FROM REFORMULATED GASOLINE

\begin{tabular}{|c|c|c|c|c|c|c|c|c|c|c|c|c|}
\hline \multirow[b]{4}{*}{ Compound } & \multicolumn{12}{|c|}{ Operating Conditions } \\
\hline & \multicolumn{4}{|c|}{ Lean } & \multicolumn{4}{|c|}{ Stolchiometric } & \multicolumn{4}{|c|}{ Rich } \\
\hline & \multicolumn{2}{|c|}{$\begin{array}{l}\text { Without } \\
\text { Catalyst }\end{array}$} & \multicolumn{2}{|c|}{$\begin{array}{c}\text { With } \\
\text { Catalyst }\end{array}$} & \multicolumn{2}{|c|}{$\begin{array}{l}\text { Without } \\
\text { Catalyst }\end{array}$} & \multicolumn{2}{|c|}{$\begin{array}{c}\text { With } \\
\text { Catalyst }\end{array}$} & \multicolumn{2}{|c|}{$\begin{array}{l}\text { Without } \\
\text { Catalyst }\end{array}$} & \multicolumn{2}{|c|}{$\begin{array}{c}\text { With } \\
\text { Catalyst }\end{array}$} \\
\hline & $\begin{array}{c}\mathrm{FTP} \\
\mathrm{mg} / \mathrm{mi}\end{array}$ & $\begin{array}{c}\text { NMOG } \\
\text { wt \% }\end{array}$ & $\begin{array}{c}\text { FTP } \\
\mathrm{mg} / \mathrm{mi}\end{array}$ & $\begin{array}{l}\text { NMOG } \\
\text { wt \% }\end{array}$ & $\begin{array}{c}\text { FTP } \\
\mathrm{mg} / \mathrm{ml}\end{array}$ & $\begin{array}{c}\text { NMOG } \\
w t \% \\
\end{array}$ & $\begin{array}{l}\text { FTP } \\
\mathrm{mg} / \mathrm{mi}\end{array}$ & $\begin{array}{c}\text { NMOG } \\
\text { wt \% } \\
\end{array}$ & $\begin{array}{l}\text { FTP } \\
\mathrm{mg} / \mathrm{mi}\end{array}$ & $\begin{array}{c}\text { NMOG } \\
\text { wt \% }\end{array}$ & $\begin{array}{c}\mathrm{FTP} \\
\mathrm{mg} / \mathrm{mi}\end{array}$ & $\begin{array}{c}\text { NMOG } \\
\text { wt \% }\end{array}$ \\
\hline METHANE & 87.7 & & 57.0 & & 76.2 & & 33.1 & & 365.8 & & 355.6 & \\
\hline ETHANE & 63.4 & 0.6 & 7.1 & $3: 8$ & 31.0 & 1.3 & 4.6 & 2.4 & 32.2 & .0 .9 & 38.4 & 1.1 \\
\hline ETHYLENE & 442.1 & 3.9 & 12.1 & 6.4 & 173.0 & 7.2 & 12.6 & 6.7 & 260.4 & 7.2 & 385.8 & 10.7 \\
\hline PROPANE & 3.6 & 20.1 & 0.2 & 0.1 & .6 & .0 .1 & 0.3 & 0.2 & 2.5 & 0.1. & 27 & 0.1. \\
\hline PROPYLENE & 441.5 & 3.9 & 8.6 & 4.6 & 141.1 & 5.9 & 8.8 & 4.7 & 134.3 & 3.7 & 146.7 & 4.1 \\
\hline ACETYUENE & 828 & 0.7 & 4.6 & 24 & 93.4 & (1.9. & 5.4 & .2 .9 & 319.1 & 8.8. & 91.3 & 2.5 \\
\hline PROPADIENE & ND & ND & ND & ND & ND & ND & ND & ND & ND & ND & ND & ND \\
\hline BUTANE: & 1361 & 12 & 18 & 0.9 & 18.6 & 0.8 & 20 & 1.1.1. & 300 & .0 .8 & 20.8. & 0.8. \\
\hline TRANS-2-BUTENE & 44.5 & 0.4 & 0.7 & 0.4 & 11.6 & 0.5 & 0.8 & 0.4 & 10.4 & 0.3 & 12.5 & 0.3 \\
\hline T.BUTENE & 61.5 & 0.5 & 1.1 & .06 & 17.4 & 07 & 1.1 & 0.6 & 145 & 0.4 & 18.0 & 0.5 \\
\hline 2-METHYLPROPENE (ISOBUTYLENE) & 473.0 & 4.2 & 9.2 & 4.9 & 130.7 & 5.4 & 10.1 & 5.4 & 124.3 & 3.4 & 240.0 & 6.7 \\
\hline 2,2 DIMETHYLPROPANE (NEOPENTANE) & 1.6.6. & 80.1 & 0.1 & .20 .1 & 0.6 & 20.1 & 0.1 & 201 & 1.1 & 2.0 .1 & 1.2 & .20 .1$. \\
\hline PROPYNE & ND & ND & ND & ND & ND & ND & ND & ND & ND & ND & ND & ND \\
\hline 1,3BUTADIENE & 617 & 0.5 & 12 & 0.6 & 213 & 0.9 & 1.1. & 0.6. & 18.2 & 0.5 & 18.7 & 0.5 \\
\hline 2-METHYLPROPANE (ISOBUTANE) & 5.2 & $<0.1$ & 0.1 & 0.1 & 1.4 & 0.1 & 0.1 & $<0.1$ & 1.3 & $<0.1$ & 2.1 & 0.1 \\
\hline 1.BUTYNE & ND. & NB & ND. & NB. & ND & ND & NO & ND & NB & No & ND & No \\
\hline METHANOL & 132.9 & 1.2 & ND & ND & 35.1 & 1.5 & ND & ND & 29.0 & 0.8 & 71.5 & 2.0 \\
\hline TTENE: & 32.5 & 0.3 & 0.7 & 0.4 & 8.5 & 0.4 & 0.6 & 0.3 & 78 & 0.2 & 116 & 0.3 \\
\hline 3-METHYL-1-BUTENE & ND & ND & ND & ND & ND & ND & ND & ND & ND & ND & ND. & ND \\
\hline ETHANOL & NO & ND & ND & NB & ND & ND & $=\mathrm{ND}$ & No & NB & ND & $=\mathrm{ND}$ & ND \\
\hline 2-METHYLBUTANE (ISOPENTANE) & 606.4 & 5.4 & 7.6 & 4.0 & 89.1 & 3.7 & 9.6 & 5.1 & 146.8 & 4.0 & 143.3 & 4.0 \\
\hline 2-BUTYNE & 33.5 & 0.3 & ND & ND. & 0.3 & $<0.1$ & $=$ ND & ND & 0.03 & $20.1 \%$ & 0.3. & $<0.1$ \\
\hline 1-PENTENE & 26.6 & 0.2 & ND & ND & 4.0 & 0.2 & 0.1 & $<0.1$ & 4.3 & 0.1 & 5.2 & 0.1 \\
\hline 2NETHYL - - BUTENE & .56 .5 & 0.5 & 0.6 & 0.3 & 1188 & 0.5 & 0.6 & 0.3 & 111 & 0.3 & 102 & 0.3 \\
\hline PENTANE & 105.7 & 0.9 & 1.4 & 0.7 & 15.4 & 0.6 & 1.6 & 0.8 & 25.5 & 0.7 & 25.8 & 0.7 \\
\hline 2. VETHYL- 1,3 BUTADIENE & $37 \%$ & 0.3 & 07 & 0.3 & 113 & 0.5 & 0.6 & 0.3 & 9.6 & 0.3 & 9.4 & 0.3 \\
\hline TRANS-2-PENTENE & 29.7 & 0.3 & 0.3 & 0.2 & 5.3 & 0.2 & 0.3 & 0.2 & 6.7 & 0.2 & 7.5 & 0.2 \\
\hline 3.3-DIMETHYL-1.BUTENE & 4.1 .1 & $<01$ & ND & ND & 0.9 & 201 & ND & NB & 06 & $<0.1$ & 0.6 & $<0.1$ \\
\hline CIS-2-PENTENE & 17.0 & 0.2 & 0.2 & 0.1 & 3.0 & 0.1 & 0.2 & 0.1 & 4.0 & 0.1 & 4.3 & 0.1 \\
\hline 2.METHYM 2. BIITENE & 70,3 & 0.6 & 114 & n 6. & 1.0 .9 & On & 1.10 & 0.5. & 165 & 10.6 & 244 & 0.6. \\
\hline
\end{tabular}


TABLE K-1 (CONT'D). SUMMARY OF SPECIATED EXHAUST EMISSIONS FROM REFORMULATED GASOLINE

\begin{tabular}{|c|c|c|c|c|c|c|c|c|c|c|c|c|}
\hline \multirow[b]{4}{*}{ Compound } & \multicolumn{12}{|c|}{ Operating Conditions } \\
\hline & \multicolumn{4}{|c|}{ Lean } & \multicolumn{4}{|c|}{ Stoichiometric } & \multicolumn{4}{|c|}{ Rich } \\
\hline & \multicolumn{2}{|c|}{$\begin{array}{l}\text { Without } \\
\text { Catalyst }\end{array}$} & \multicolumn{2}{|c|}{$\begin{array}{c}\text { With } \\
\text { Catalyst }\end{array}$} & \multicolumn{2}{|c|}{$\begin{array}{l}\text { Without } \\
\text { Catalyst }\end{array}$} & \multicolumn{2}{|c|}{$\begin{array}{c}\text { With } \\
\text { Catalyst }\end{array}$} & \multicolumn{2}{|c|}{$\begin{array}{l}\text { Without } \\
\text { Catalyst }\end{array}$} & \multicolumn{2}{|c|}{$\begin{array}{c}\text { With } \\
\text { Catalyst }\end{array}$} \\
\hline & $\begin{array}{c}\text { FTP } \\
\mathrm{mg} / \mathrm{mi}\end{array}$ & $\begin{array}{c}\text { NMOG } \\
\text { wt \% }\end{array}$ & $\begin{array}{c}\text { FTP } \\
\mathrm{mg} / \mathrm{mi}\end{array}$ & $\begin{array}{c}\text { NMOG } \\
\text { wt \% } \\
\end{array}$ & $\begin{array}{c}\text { FTP } \\
\mathrm{mg} / \mathrm{mi}\end{array}$ & $\begin{array}{c}\text { NMOG } \\
\text { wt \% } \\
\end{array}$ & $\begin{array}{c}\mathrm{FTP} \\
\mathrm{mg} / \mathrm{mi}\end{array}$ & $\begin{array}{c}\text { NMOG } \\
\text { wt \% } \\
\end{array}$ & $\begin{array}{c}\begin{array}{r}\text { FTP } \\
\mathrm{mg} / \mathrm{mi}\end{array} \\
\end{array}$ & $\begin{array}{c}\text { NMOG } \\
\text { wt } \% \\
\end{array}$ & $\begin{array}{c}\text { FTP } \\
\mathrm{mg} / \mathrm{mi}\end{array}$ & $\begin{array}{c}\text { NMOG } \\
\text { wt \% } \\
\end{array}$ \\
\hline CYCLOPENTADIENE & 15.6 & 0.1 & 0.4 & 0.2 & 7.2 & 0.3 & 0.4 & 0.2 & 13.0 & 0.4 & 6.4 & 0.2 \\
\hline 2,2 DIMETHYLBUTANE & $35: 0$ & 0.3 & 0.6 & 0.3 & 61 & $=0.3$ & 07 & 0.4 & 97 & 0.3 & 9.0 & 0.3 \\
\hline CYCLOPENTENE & 8.1 & 0.1 & 0.1 & 0.1 & 2.8 & 0.1 & 0.1 & $<0.1$ & 2.7 & 0.1 & 2.2 & 0.1 \\
\hline 4. METHYL- 1 PENTENE & 328 & $=0,3$ & 0.1 & .0 .11$. & 1.9 & 0.1 & 0.1 & 01 & 20 & 0.1 & 20 & 0.1 \\
\hline 3-METHYL-1-PENTENE & 15.8 & 0.1 & 0.1 & $<0.1$ & 4.5 & 0.2 & 0.1 & $<0.1$ & 2.8 & 0.1 & 1.5 & $<0.1$ \\
\hline CYCLOPENTANE & ND. & NB & ND & No & ND & ND & No & NO & ND & ND. & ND & ND \\
\hline 2,3-DIMETHYLBUTANE & 116.6 & 1.0 & 1.5 & 0.8 & 19.1 & 0.8 & 1.8 & 1.0 & 29.0 & 0.8 & 29.6 & 0.8 \\
\hline MTBE & 838.7 & 17.4 & 4.5 & 2.4 & 130.2 & 5.4 & $=3.8$ & 20 & 2253 & 62 & 72.5 & 2.0 \\
\hline 2,3-DIMETHYL-1-BUTENE & ND & ND & ND & ND & ND & ND & ND & ND & ND & ND & ND & ND \\
\hline 4METHYL CIS 2 PENTENE & No & NO & NO & No & No & $\mathrm{ND}$ & No & No & NO & NO & $1 . \mathrm{NB}$ & ND. \\
\hline 2-METHYLPENTANE & 4.1 & $<0.1$ & 2.6 & 1.4 & 0.2 & $<0.1$ & 3.1 & 1.7 & 2.3 & 0.1 & 2.1 & 0.1 \\
\hline 4. NETHYL TRANS 2-PENTENE & ND. & ND & No & ND & ND & No. & 1. NB & No. & No & $\mathrm{ND}$ & ND & ND \\
\hline 3-METHYLPENTANE & 116.0 & 1.0 & 1.6 & 0.9 & 17.4 & 0.7 & 1.8 & 1.0 & 30.2 & 0.8 & 30.8 & 0.9 \\
\hline 2.METHYL 1 PENTENE & 14.8 & .0 .11 & 0.1. & $=.01$ & 1.9 & 0.1 & 0.1 & .01 & 3.0 & 0.1 & 28.8 & 0.1 \\
\hline 1-HEXENE & 9.7 & 0.1 & 0.2 & 0.1 & 1.6 & 0.1 & 0.1 & 0.1 & 1.7 & $<0.1$ & 1.8 & $<0.1$ \\
\hline HEXANE & 70.3 & 0.6 & 1.0 & 0.5 & 10.4 & 0.4. & 1.1 & 0.6 & 18.8 & 05 & 19.3 & 0.5 \\
\hline TRANS-3-HEXENE & 10.1 & 0.1 & 0.1 & 0.1 & 1.5 & 0.1 & 0.1 & 0.1 & 2.4 & 0.1 & 2.5 & 0.1 \\
\hline CIS3.HEXENE & 176 & 0.2 & 02 & $0.1 \%$ & 29 & 0.1 .9 & 0.2 & 0.1 & 4.1 & 011 & .4 .3 & 0.1 \\
\hline TRANS-2-HEXENE & ND & ND & ND & ND & ND & ND & ND & ND & ND & ND & ND & ND \\
\hline 3.METHYL TRANS 2-PEI & 221 & 0.2 & 0.2 & 0.1 & 3.7 & 0.2 & 0.2 & 0.1 & 5.4 & 0.1 & 5.7 & 02 \\
\hline 2-METHYL-2-PENTENE & 16.0 & 0.1 & 0.2 & 0.1 & 2.9 & 0.1 & 0.2 & 0.1 & 3.9 & 0.1 & 3.8 & 0.1 \\
\hline 3 METHYLO YCLOPENTENE & ND & ND. & $\mathrm{ND}$ & ND & No & NO & NO & NO & ND & ND & ND & $\mathrm{NB}$ \\
\hline CIS-2-HEXENE & 8.4 & 0.1 & 0.1 & 0.1 & 1.3 & 0.1 & 0.1 & $<0.1$ & 2.1 & 0.1 & 2.2 & 0.1 \\
\hline ETBE & NB & No & ND & NO & No & NO & ND & $\mathrm{ND}$ & ND. & ND & ND & NB \\
\hline 3-METHYL-CIS-2-PENTENE & 17.8 & 0.2 & 0.2 & 0.1 & 3.1 & 0.1 & 0.2 & 0.1 & 4.6 & 0.1 & 4.9 & 0.1 \\
\hline 2,2-DIMETHYLPENTANE & 44.9 & 0.4 & 0.6 & 0.3 & 6.3 & 0.3 & .0 .7 & .0 .3 & 114 & 0.3 & 11.6 & 0.3 \\
\hline METHYLCYCLOPENTANE & 8.4 & 0.1 & 0.1 & $<0.1$ & 1.7 & 0.1 & 0.1 & $<0.1$ & 1.4 & $<0.1$ & 1.3 & $<0.1$ \\
\hline 2,4 DIMETHYLPENTANE & 216.5 & 1.9 & 3.0. & 1,6 & 33.5 & 1.4 & .3 .4 & 1,8 & 581 & 1.6 & 60.0 & $1 \%$ \\
\hline 2,3,3-TRIMETHYL-1-BUTENE & ND & ND & ND & ND & ND & ND & ND & ND & ND & ND & ND & ND \\
\hline 2.2.3.TRIMETHYLBUTANE. & 1.5 .0 & 20.1 & $=0.2$ & 011 & 0.7 & $<0.1$ & 0.2 & $=.01$ & $=3.0$ & 0.1 & 2.27 & 0.1. \\
\hline
\end{tabular}


TABLE K-1 (CONT'D). SUMMARY OF SPECIATED EXHAUST EMISSIONS FROM REFORMULATED GASOLINE

\begin{tabular}{|c|c|c|c|c|c|c|c|c|c|c|c|c|}
\hline \multirow[b]{4}{*}{ Compound } & \multicolumn{12}{|c|}{ Operating Conditions } \\
\hline & \multicolumn{4}{|c|}{ Lean } & \multicolumn{4}{|c|}{ Stoichiometric } & \multicolumn{4}{|c|}{ Rich } \\
\hline & \multicolumn{2}{|c|}{$\begin{array}{l}\text { Without } \\
\text { Catalyst }\end{array}$} & \multicolumn{2}{|c|}{$\begin{array}{c}\text { With } \\
\text { Catalyst }\end{array}$} & \multicolumn{2}{|c|}{$\begin{array}{l}\text { Without } \\
\text { Catalyst }\end{array}$} & \multicolumn{2}{|c|}{$\begin{array}{c}\text { With } \\
\text { Catalyst }\end{array}$} & \multicolumn{2}{|c|}{$\begin{array}{l}\text { Without } \\
\text { Catalyst }\end{array}$} & \multicolumn{2}{|c|}{$\begin{array}{c}\text { With } \\
\text { Catalyst }\end{array}$} \\
\hline & $\begin{array}{c}\text { FTP } \\
\mathrm{mg} / \mathrm{mi}\end{array}$ & $\begin{array}{c}\text { NMOG } \\
\text { wt \% }\end{array}$ & $\begin{array}{c}\text { FTP } \\
\mathrm{mg} / \mathrm{ml}\end{array}$ & $\begin{array}{c}\text { NMOG } \\
\text { wt \% } \\
\end{array}$ & $\begin{array}{c}\text { FTP } \\
\mathrm{mg} / \mathrm{mi}\end{array}$ & $\begin{array}{c}\text { NMOG } \\
\text { wt \% }\end{array}$ & $\begin{array}{c}\text { FTP } \\
\mathrm{mg} / \mathrm{mi}\end{array}$ & $\begin{array}{c}\text { NMOG } \\
\text { wt \% } \\
\end{array}$ & $\begin{array}{c}\text { FTP } \\
\mathrm{mg} / \mathrm{ml}\end{array}$ & $\begin{array}{c}\text { NMOG } \\
\text { wt \% } \\
\end{array}$ & $\begin{array}{c}\text { FTP } \\
\mathrm{mg} / \mathrm{ml}\end{array}$ & $\begin{array}{c}\text { NMOG } \\
\text { wt \% } \\
\end{array}$ \\
\hline 3,4-DIMETHYL-1-PENTENE & 3.4 & $<0.1$ & $<0.1$ & $<0.1$ & 0.6 & $<0.1$ & $<0.1$ & $<0.1$ & 0.8 & $<0.1$ & 0.9 & $<0.1$ \\
\hline 1 METHYLCYCLOPENTENE & 11.9 & $.0 .1 \%$ & 2.80 .1 & .00 .1 & 1.6 & 0.1 & 0.2 & 0.1 & 3.6 & $0.1 \%$ & 3.8 & 0.1 \\
\hline BENZENE & 168.5 & 1.5 & 7.2 & 3.8 & 63.4 & 2.6 & 7.9 & 4.2 & 120.0 & 3.3 & 126.3 & 3.5 \\
\hline 3-METHYL-1 НHEXENE & No. & $=\mathrm{NQ}$ & ND & No & ND. & ND & NB. & ND & ND & ND & No & 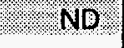 \\
\hline 3,3-DIMETHYLPENTANE & 7.1 & 0.1 & 0.1 & 0.1 & 2.5 & 0.1 & 0.1 & $<0.1$ & 2.3 & 0.1 & 2.2 & 0.1 \\
\hline CYCLOHEXANE: & 2.25 & 20.1 & 0.1. & $\% .2011 ?$ & 0.5 & .20 .1 & .0 .1 & $=20.1$ & 0.6 & .20 .1 & 0.5 & 20.1 \\
\hline 2-METHYLHEXANE & ND & ND & ND & ND & ND & ND & ND & ND & ND & ND & ND & ND \\
\hline 2,3-DIMETHYLPENTANE & 4402 & 3.9 & 5:9. & 3.1 & 66.2 & 2.8 & 70 & 3.7 & 1194 & 3.3 & 122.0 & $=3.4$ \\
\hline 1,1-DIMETHYLCYCLOPENTANE & ND & ND & ND & ND & ND & ND & ND & ND & ND & ND & ND & ND \\
\hline CYCLOHEXENE: & 1.3 .6 & $=0.11$ & 80.1 & .20 .11 & 0.6 & 20.1 & $2 \times 0.1$ & $\div<0.1$ & 0.6 & 2.801 & 0.6 & 20.1 \\
\hline 3-METHYLHEXANE & 96.5 & 0.9 & 1.3 & 0.7 & 14.3 & 0.6 & 1.5 & 0.8 & 27.0 & 0.7 & 25.5 & 0.7 \\
\hline CIS-1,3 DIMETHYLCYCLO & 11.6 & .0 .1 & 0.1 & $0.1 \%$ & 1.6 & 0.1 & .0 .1 & 0.1. & 3.0 & 0.1. & 3.0 & 0.1. \\
\hline 3-ETHYLPENTANE & ND & ND & ND & ND & ND & ND & ND & ND & ND & ND & ND & ND \\
\hline TRANS-12-DIMETHYLCYCLOPE & ND. & ND. & $.2 \mathrm{ND}$ & No & ND & 1.20 & ND & ND & ND & $\mathrm{NB}$ & ND & NB \\
\hline TRANS-1,3-DIMETHYLCYCLOPEN & ND & ND & ND: & ND & ND & ND & ND & ND & ND & ND & ND & ND \\
\hline 1.HEPTENE & ND & NO & ND. & ND & NB & 1.: ND. 1 & . ND & 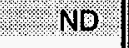 & NO & ND. & . ND & ND. \\
\hline 2,2,4-TRIMETHYLPENTANE & 763.0 & 6.8 & 11.3 & 6.0 & 120.7 & 5.0 & 12.9 & 6.9 & 214.3 & 5.9 & 218.5 & 6.1 \\
\hline 2-METHYL-1 HEXENE & $\mathrm{ND}$ & $.1 . \mathrm{ND}$ & ND. & No: & No & ND. & ND & ND & ND. & $.2 \mathrm{ND}$ & ND & No: \\
\hline TRANS-3-HEPTENE & ND & ND & ND & ND & ND & ND & ND & ND & ND & ND & ND & ND \\
\hline HEPTANE : & 66.0 & 0.6 & 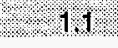 & .0 .6 & 10.3 & 0.4 & 10 & 0.6 & 19.1 & 0.5 & 19.6 & 0.5 \\
\hline 2-METHYL-2-HEXENE & 14.0 & 0.1 & 0.2 & 0.1 & 1.9 & 0.1 & 0.2 & 0.1 & 3.8 & 0.1 & 4.0 & 0.1 \\
\hline 3-METHYL-TPANS-3. HEXENE & $=\mathrm{ND}$ & . ND & ND & ND. & ND & ND & No & $\mathrm{NB}$ & ND & ND & ND & ND \\
\hline TRANS-2-HEPTENE & 7.5 & 0.1 & 0.1 & $<0.1$ & 1.2 & 0.1 & 0.1 & $<0.1$ & 2.0 & 0.1 & 1.8 & 0.1 \\
\hline 3-ETHYL-GIS-2-PENTENE & 1.9 & 20.1 & NB & $\mathrm{NB}$ & 0.3 & 20.1 & ND & ND & 0.4 & 201. & 0.5 & 20.1 \\
\hline 2,4,4-TRIMETHYL-1-PENTENE & 10.1 & 0.1 & 0.1 & 0.1 & 1.5 & 0.1 & 0.1 & 0.1 & 2.6 & 0.1 & 2.3 & 0.1 \\
\hline 2,2,4-TAIMETHYL-I.PENTENE & No & ND & ND & ND & ND & NB & NB. & ND & ND & ND & ND & No. \\
\hline 2,3-DIMETHYL-2-PENTENE & ND & ND & ND & ND & ND & ND & ND & ND & ND & ND: & ND & ND \\
\hline CIS-2HEPTENE & 7.6 & 0.1 & 0.1 & 0.1 & 1.4 & 0.1 & 0.1 & 0.1 & 2.0 & 0.1 & 21 & 0.1 \\
\hline METHYLCYCLOHEXANE & 6.7 & 0.1 & 0.1 & 0.1 & 1.1 & $<0.1$ & 0.1 & 0.1 & 2.0 & 0.1 & 2.1 & 0.1 \\
\hline CIS 1,2.DIMETHYLOYCLOPENTANE & NO & ND & NDE & NO & ND & NE. & $1 . \mathrm{ND}$ & ND & ND. & ND. & $2 . \mathrm{NB}$ & ND. \\
\hline
\end{tabular}


TABLE K-1 (CONT'D). SUMMARY OF SPECIATED EXHAUST EMISSIONS FROM REFORMULATED GASOLINE

\begin{tabular}{|c|c|c|c|c|c|c|c|c|c|c|c|c|}
\hline \multirow[b]{4}{*}{ Compound } & \multicolumn{12}{|c|}{ Operating Conditions } \\
\hline & \multicolumn{4}{|c|}{ Lean } & \multicolumn{4}{|c|}{ Stoichiometric } & \multicolumn{4}{|c|}{ Rich } \\
\hline & \multicolumn{2}{|c|}{$\begin{array}{l}\text { Without } \\
\text { Catalyst }\end{array}$} & \multicolumn{2}{|c|}{$\begin{array}{c}\text { With } \\
\text { Catalyst }\end{array}$} & \multicolumn{2}{|c|}{$\begin{array}{l}\text { Without } \\
\text { Catalyst }\end{array}$} & \multicolumn{2}{|c|}{$\begin{array}{c}\text { With } \\
\text { Catalyst }\end{array}$} & \multicolumn{2}{|c|}{$\begin{array}{l}\text { Without } \\
\text { Catalyst }\end{array}$} & \multicolumn{2}{|c|}{$\begin{array}{c}\text { With } \\
\text { Catalyst }\end{array}$} \\
\hline & $\begin{array}{c}\text { FTP } \\
\mathrm{mg} / \mathrm{mi}\end{array}$ & $\begin{array}{c}\text { NMOG } \\
\text { wt \% }\end{array}$ & $\begin{array}{l}\text { FTP } \\
\mathrm{mg} / \mathrm{mi}\end{array}$ & $\begin{array}{c}\text { NMOG } \\
\text { wt \% }\end{array}$ & \begin{tabular}{|c|} 
FTP \\
$\mathrm{mg} / \mathrm{mi}$
\end{tabular} & $\begin{array}{c}\text { NMOG } \\
\text { wt \% }\end{array}$ & $\begin{array}{c}\text { FTP } \\
\mathrm{mg} / \mathrm{ml}\end{array}$ & $\begin{array}{l}\text { NMOG } \\
\text { wt \% }\end{array}$ & $\begin{array}{c}\text { FTP } \\
\mathrm{mg} / \mathrm{mi}\end{array}$ & $\begin{array}{c}\text { NMOG } \\
\text { wt \% }\end{array}$ & $\begin{array}{c}\text { FTP } \\
\mathrm{mg} / \mathrm{mi}\end{array}$ & $\begin{array}{c}\text { NMOG } \\
\text { wt } \%\end{array}$ \\
\hline 2,2-DIMETHYLHEXANE & 78.1 & 0.7 & 1.2 & 0.6 & 12.5 & 0.5 & 1.4 & 0.8 & 23.1 & 0.6 & 24.2 & 0.7 \\
\hline (1.1.3.TRIMETHYLOYCLOPENTANE & NO & ND & ND & ND & ND &. $\mathrm{ND}$ & ND & ND & ND. & ND: & No & NO \\
\hline 2,4,4-TRIMETHYL-2-PENTENE & 2.9 & $<0.1$ & ND & ND & ND & ND & ND & ND & ND & ND & 2.0 & 0.1 \\
\hline 2,2,3.TRIMETHYLPENTANE & ND & ND & ND & No & No & NB & ND & $\mathrm{NB}$ & ND. & No & ND & NB: \\
\hline 2,5-DIMETHYLHEXANE & ND & ND & ND & ND & ND & ND & ND & ND & ND & ND & ND & ND \\
\hline ETHYLCYCLOPENTANE & ND. & ND. & No: & ND. & ND & ND & ND & ND & ND & ND. & NB & NB \\
\hline 2,4-DIMETHYLHEXANE & 98.0 & 0.9 & 1.5 & 0.8 & 15.3 & 0.6 & 1.7 & 0.9 & 28.6 & 0.8 & 29.1 & 0.8 \\
\hline 1.TRANS-2.CIS-4TIMETHYLCYCLOPENTANE & NO & ND. & . ND. & .0 .10$. & NB & NO & ND & ND & NO & ND & ND & NB \\
\hline 3,3-DIMETHYLHEXANE & 9.8 & 0.1 & 0.1 & 0.1 & 1.6 & 0.1 & 0.2 & 0.1 & 2.8 & 0.1 & 2.9 & 0.1 \\
\hline 1-TRANS-2-O1S-3-TRIMETHYLOYCLOPENTANE & ND & $1 . \mathrm{NO}$ & $\mathrm{NB}$ & NO & NB & No & No & ND & NO & ND. & ND & No \\
\hline 2,3,4-TRIMETHYLPENTANE & ND & ND & ND & ND & ND & ND & ND & ND & ND & ND & ND & ND \\
\hline 2,3,3-TRIMETHYLPENTANE & 176.9 & 1.6 & 2.4 & 13 & 26.2 & $1.1 \%$ & 30 & 186 & 49.0 & $=1.3$ & 50.3 & 1.4 \\
\hline TOLUENE & 842.1 & 7.5 & 16.1 & 8.5 & 181.4 & 7.6 & 16.5 & 8.8 & 287.4 & 7.9 & 293.3 & 8.2 \\
\hline 2.3.DIMETHYLHEXANE & 176.2 & 1.6 & 2.0 & 1.1 & 241 & 10 & 20 & 11. & 456 & 1.3 & 45.6 & 1.3 \\
\hline 1,1,2-TRIMETHYLCYCLOPENTANE & ND & ND & ND & ND. & ND & ND & ND & ND & ND & ND & ND & ND \\
\hline 2METHYLHEPTANE & 53.2 & 0.5 & 0.8 & 0.4 & 7.9 .9 & 0.3 & 0.9 & 0.5 & 16.6 & 0.5 & 16.9 & 0.5 \\
\hline 3,4-DIMETHYLHEXANE & ND & ND & ND & ND & ND & ND & ND & ND & NO & NO & ND & ND \\
\hline $2,2,4,4$ TETRAMETHYLPENTANE & ND & ND & No & No & NO & NB &. $\mathrm{ND}$ & NB & NO & ND & ND & NO \\
\hline 4-METHYLHEPTANE & 16.7 & 0.1 & 0.2 & 0.1 & 2.6 & 0.1 & 0.3 & 0.1 & 5.3 & 0.1 & 5.4 & 0.1 \\
\hline 2-METHYL-3-ETHYLPENTANE & $\mathrm{NB}$ & ND & ND. & ND & ND & NO & ND. & ND & No & ND & ND & ND \\
\hline 2,6-DIMETHYLHEPTANE & ND & ND & ND & ND & ND & ND & ND & ND & ND & ND & ND & ND \\
\hline 3 METHYLHEPTANE & 74.2 & .0 .7 & 1.1 .1$. & 0.6 & 11.7 & 0.5 & 1.3 & 0.7 & 237 & 0.6 & 23.9 & 0.7. \\
\hline 1-CIS,2-TRANS,3-TRIMETHYLCYGLOPENTANE & ND & ND & ND & ND & ND & ND & ND & ND & ND & ND & ND & ND \\
\hline CIS. 1,3 DIMETHYLCYCLOHEXANE & No. & No & ND & No: & No & ND & ND. & ND. & $\mathrm{NB}$ & ND & $\mathrm{NB}$ & ND \\
\hline TRANS-1,4-DIMETHYLCYCLOHEXANE & 103.0 & 0.9 & 1.6 & 0.9 & 16.9 & 0.7 & 1.9 & 1.0 & 32.1 & 0.9 & 32.6 & 0.9 \\
\hline 3ETHYLHEXANE ? & ND. & NO & ND. & ND & ND. & ND & ND. & NO & ND. & ND & ND & $=\mathrm{ND}$ ? \\
\hline 2,2,5-TRIMETHYLHEXANE & 6.2 & 0.1 & 0.1 & $<0.1$ & 0.9 & $<0.1$ & 0.1 & $<0.1$ & 1.8 & 0.0 & 1.8 & 0.1 \\
\hline CIS - METHYL-3ETHYLCYCLOPENTANE & NB & ND & ND. & ND & ND & No & ND & ND. & ND & ND. & ND & No: \\
\hline 1,1-DIMETHYLCYCLOHEXANE & ND & ND & ND & ND & ND & ND & ND & ND & ND & ND & ND & ND \\
\hline TRANS 1 METHYL 2.ETHYLCYCLOPENTANE & $\mathrm{NO}$ & $\mathrm{NB}$ & $=2 \mathrm{NB}$ & ND & ND & $\mathrm{ND}$ & ND & $\mathrm{ND}$ & ND & ND 1 & $1 . \mathrm{NB}$ & $\mathrm{NB}$ \\
\hline
\end{tabular}


TABLE K-1 (CONT'D). SUMMARY OF SPECIATED EXHAUST EMISSIONS FROM REFORMULATED GASOLINE

\begin{tabular}{|c|c|c|c|c|c|c|c|c|c|c|c|c|}
\hline \multirow[b]{4}{*}{ Compound } & \multicolumn{12}{|c|}{ Operating Conditions } \\
\hline & \multicolumn{4}{|c|}{ Lean } & \multicolumn{4}{|c|}{ Stoichiometric } & \multicolumn{4}{|c|}{ Rich } \\
\hline & \multicolumn{2}{|c|}{$\begin{array}{l}\text { Without } \\
\text { Catalyst }\end{array}$} & \multicolumn{2}{|c|}{$\begin{array}{c}\text { With } \\
\text { Catalyst }\end{array}$} & \multicolumn{2}{|c|}{$\begin{array}{l}\text { Without } \\
\text { Catalyst }\end{array}$} & \multicolumn{2}{|c|}{$\begin{array}{c}\text { With } \\
\text { Catalyst }\end{array}$} & \multicolumn{2}{|c|}{$\begin{array}{l}\text { Without } \\
\text { Catalyst }\end{array}$} & \multicolumn{2}{|c|}{$\begin{array}{c}\text { With } \\
\text { Catalyst }\end{array}$} \\
\hline & $\begin{array}{c}\text { FTP } \\
\mathrm{mg} / \mathrm{mi}\end{array}$ & $\begin{array}{c}\text { NMOG } \\
\text { wt \% }\end{array}$ & $\begin{array}{c}\text { FTP } \\
\mathrm{mg} / \mathrm{mi}\end{array}$ & $\begin{array}{c}\text { NMOG } \\
\text { wt \% }\end{array}$ & $\begin{array}{c}\text { FTP } \\
\mathrm{mg} / \mathrm{mi}\end{array}$ & $\begin{array}{c}\text { NMOG } \\
\text { wt \% }\end{array}$ & $\begin{array}{c}\text { FTP } \\
\mathrm{mg} / \mathrm{mi}\end{array}$ & $\begin{array}{c}\text { NMOG } \\
\text { wt \% }\end{array}$ & $\begin{array}{c}\text { FTP } \\
\mathrm{mg} / \mathrm{mi}\end{array}$ & $\begin{array}{c}\text { NMOG } \\
\text { wt \% }\end{array}$ & $\begin{array}{l}\mathrm{FTP} \\
\mathrm{mg} / \mathrm{mi}\end{array}$ & $\begin{array}{c}\text { NMOG } \\
\text { wt \% }\end{array}$ \\
\hline 1-METHYL-1-ETHYL-CYCLOPENT & ND & ND & ND & ND & ND & ND & ND & ND & ND & ND & ND & ND \\
\hline 2,4,4-TRIMETHYLLEXANE & ND & $=$ ND & $\mathrm{ND}$ & ND. & NB & ND & NB & ND & $\mathrm{ND}$ & ND. & ND & ND \\
\hline 2,2,4-TRIMETHYLHEXANE & ND & ND & ND & ND & ND & ND & ND & ND & ND & ND & ND & ND \\
\hline TRANS 1,2 DIMETHYLOYCLOHE & NB & NO & ND & ND. & ND & ND & ND. & ND & ND & ND. & ND & ND \\
\hline 1-OCTENE & 5.5 & $<0.1$ & 0.1 & $<0.1$ & 1.2 & $<0.1$ & 0.1 & 0.1 & 1.9 & 0.1 & 2.0 & 0.1 \\
\hline TRANS 4 -OCTENE & 5.3. & $<0.1$ & 0.1 & 2.80 .1 & 0.9 & 2.1 .1 & 0.1 & 80.1 & 1.7 & 20,1 & 1.6 & 80.1 \\
\hline OCTANE & 34.4 & 0.3 & 0.5 & 0.3 & 5.7 & 0.2 & 0.6 & 0.3 & 11.3 & 0.3 & 11.6 & 0.3 \\
\hline TRANS 2-OCTENE & $7 \%$ & 0.1 & 0.1. & 20.1 & 1,2 & 1.201 & 0.1 & 1.20 .1 & 2.83 & 0.1 & 2.4 & 0.1 . \\
\hline TRANS-1,3-DIMETHYLCYCLOHEXANE & 9.2 & 0.1 & 0.1 & $<0.1$ & 1.5 & 0.1 & 0.1 & $<0.1$ & 2.8 & 0.1 & 2.5 & 0.1 \\
\hline CIS-1,4-DIMETHYLOYOLOHEXANE & ND & 1. ND & NB. & NO & $1 . \mathrm{NB}$ & 1.0 .10 & No & ND & ND & ND & ND & NB \\
\hline CIS-2-OCTENE & 3.4 & $<0.1$ & ND & ND & 0.3 & $<0.1$ & ND & ND & 0.5 & $<0.1$ & 0.5 & $<0.1$ \\
\hline 2,3,5-TRIMETHYLHEXANE & 18.4 & 0.2 & 0.2 & 001. & 2.9 & 0.11 & 0.3 & 02 & 53 & 0.1 & 5.4 & 0.1 \\
\hline CIS-1-METHYL-2-ETHYLCYCLOPENTANE & ND & ND & ND & ND & ND & ND & ND & ND & ND & ND & ND & ND \\
\hline 2-METHYL-2 ETHYLHEPTANE & ND & ND: & ND. & ND. & ND & ND. & ND & ND & ND & No & ND & ND \\
\hline 2,4-DIMETHYLHEPTANE & 7.8 & 0.1 & 0.7 & 0.4 & 1.6 & 0.1 & 0.5 & 0.2 & 2.6 & 0.1 & 2.9 & 0.1 \\
\hline 4.4 DIMETHYLHEPTANE & ND & $=$ NO & NO & ND & ND & $1.2 \mathrm{NO}$ & $.2 \mathrm{NO}$ & ND & ND & ND & ND & ND \\
\hline CIS-1,2-DIMETHYLCYCLOHEXANE & 10.6 & 0.1 & 0.1 & 0.1 & 1.5 & 0.1 & 0.2 & 0.1 & 2.9 & 0.1 & 3.1 & 0.1 \\
\hline ETHYLCYCLOHEXANE & 177 & 0.2 & 0.2 & 01 & 2.8. & 0.1 & 0.4 & .0 .2 & 5.3 & 0.1 & 5.4. & 0.2 \\
\hline PROPYLCYCLOHEXANE & ND & ND & ND & ND & ND & ND & ND & ND & ND & ND & ND & ND \\
\hline 2METHYL-4-ETHYLHEXANE & ND & ND. & NB. & ND & NO & NO & $\mathrm{ND}$ & ND & ND & ND & NO & NB \\
\hline 2,6-DIMETHYLHEPTANE & ND & ND & ND & ND & ND & ND & ND & ND & ND & ND & ND & ND \\
\hline 1, 13-TRIMETHYLOYCLOHEXANE & ND. & $\mathrm{NB}$ & ND & ND & NB & NB & No & ND & $\mathrm{ND}$ & ND. & ND & $\mathrm{ND}$ \\
\hline 2,5-DIMETHYLHEPTANE & ND & ND & ND & ND & ND & ND & ND & ND & ND & ND & ND & ND \\
\hline 3,3.DIMETHYLHEPTANE & $\mathrm{NB}$ & NO: & $\mathrm{NB}$ & ND & $\mathrm{NB}$ & $\mathrm{NO}$ & ND & ND & ND & ND & ND & ND \\
\hline 3,5-DIMETHYLHEPTANE & ND & ND & ND & ND & ND & ND & ND & ND & ND & ND & ND. & ND \\
\hline ETHYLBENZENE & 301.0 & 2.7 & 5.3 & 2.8. & 616 & 2.6 & 4.9 & 2.6 & 960 & 2.6 & 99.3 & 28 \\
\hline 2,3-DIMETHYLHEPTANE & ND & ND & $N D$ & ND & ND & ND & ND & ND & ND & ND & ND & ND \\
\hline M- 8 P XYLENE & 662.5 & 5.9 & 13. & 6.9 & 1372 & 57 & 12.2 & 6.5 & 226.3 & 6.2 & 2326 & 6.5 \\
\hline 4-METHYLOCTANE & ND & ND & ND & ND & ND & ND & ND & ND & ND & ND & ND & ND \\
\hline 2.METHYML OCTANE & $\lcm{25.3}$ & 0.2 & 0.0 .4 & 0.2 & 4.3 & 022 & 0.5 & 0.2 & 9.0 & 0.2 & 9.2 & 0.3 \\
\hline
\end{tabular}


TABLE K-1 (CONT'D). SUMMARY OF SPECIATED EXHAUST EMISSIONS FROM REFORMULATED GASOLINE

\begin{tabular}{|c|c|c|c|c|c|c|c|c|c|c|c|c|}
\hline \multirow[b]{4}{*}{ Compound } & \multicolumn{12}{|c|}{ Operating Conditions } \\
\hline & \multicolumn{4}{|c|}{ Lean } & \multicolumn{4}{|c|}{ Stolchiometric } & \multicolumn{4}{|c|}{ Rich } \\
\hline & \multicolumn{2}{|c|}{$\begin{array}{l}\text { Without } \\
\text { Catalyst }\end{array}$} & \multicolumn{2}{|c|}{$\begin{array}{c}\text { With } \\
\text { Catalyst }\end{array}$} & \multicolumn{2}{|c|}{$\begin{array}{l}\text { Without } \\
\text { Catalyst }\end{array}$} & \multicolumn{2}{|c|}{$\begin{array}{c}\text { With } \\
\text { Catalyst }\end{array}$} & \multicolumn{2}{|c|}{$\begin{array}{l}\text { Without } \\
\text { Catalyst }\end{array}$} & \multicolumn{2}{|c|}{$\begin{array}{c}\text { With } \\
\text { Catalyst }\end{array}$} \\
\hline & $\begin{array}{c}\text { FTP } \\
\mathrm{mg} / \mathrm{mi}\end{array}$ & $\begin{array}{c}\text { NMOG } \\
\text { wt \% } \\
\end{array}$ & $\begin{array}{c}\text { FTP } \\
\mathrm{mg} / \mathrm{mi}\end{array}$ & $\begin{array}{c}\text { NMOG } \\
\text { wt } \%\end{array}$ & $\begin{array}{c}\text { FTP } \\
\mathrm{mg} / \mathrm{mi}\end{array}$ & $\begin{array}{c}\text { NMOG } \\
\text { wt \% }\end{array}$ & $\begin{array}{c}\text { FTP } \\
\mathrm{mg} / \mathrm{mi}\end{array}$ & $\begin{array}{c}\text { NMOG } \\
\text { wt \% }\end{array}$ & $\begin{array}{c}\mathrm{FTP} \\
\mathrm{mg} / \mathrm{mi}\end{array}$ & $\begin{array}{c}\text { NMOG } \\
\text { wt \% }\end{array}$ & $\begin{array}{l}\text { FTP } \\
\mathrm{mg} / \mathrm{mi}\end{array}$ & $\begin{array}{c}\text { NMOG } \\
\text { wt \% }\end{array}$ \\
\hline 3-METHYLOCTANE & 16.5 & 0.1 & 0.3 & 0.1 & 2.7 & 0.1 & 0.3 & 0.2 & 5.7 & 0.2 & 6.3 & 0.2 \\
\hline STYAENE & ND & ND & ND & ND. & . ND. & ND!? & NB & ND & ND. & $\mathrm{ND}$ & NO & No \\
\hline O-XYLENE & 219.7 & 1.9 & 4.4 & 2.4 & 44.5 & 1.9 & 4.0 & 2.1 & 72.7 & 2.0 & 76.3 & 2.1 \\
\hline 24,6-TRIMETHYLHEXANE & $1 . N B$ & ND. & ND & No & ND & ND & ND & ND & ND. & $\mathrm{NB}=$ & ND. & ND \\
\hline 1-NONENE & 23.0 & 0.2 & 0.2 & 0.1 & 4.2 & 0.2 & 0.4 & 0.2 & 8.5 & 0.2 & 8.6 & 0.2 \\
\hline NONANE: & 18.5 & 0.2 & 0.3 & 0.1 & 2.9 & 0.1 & 0.3 & 02 & 5.7 & $0.2 \div$ & 6.4. & 0.2 \\
\hline ISOPROPYLBENZENE (CUMENE) & 11.0 & 0.1 & 0.2 & 0.1 & 2.1 & 0.1 & 0.2 & 0.1 & 3.5 & 0.1 & 3.7 & 0.1 \\
\hline 2.2-DIMETHYLOCTANE & 10.3 & 0.1 & 0.1 & 2.80 .1 & 1.9 & 0.1 & 0.2 & 0.1 & 3.4 & 0.1 & 3.5 & 0.1. \\
\hline 2,4-DIMETHYLOCTANE & 50.6 & 0.4 & 1.0 & 0.5 & 10.8 & 0.5 & 0.3 & 0.1 & 8.1 & 0.2 & 5.6 & 0.2 \\
\hline HPROPYLBENZENE & 33.4 & 0.3 & $=0.3$ & 0.1 & 6.4 & 0.3 & 0.5 & 0.03 & 11.3 & 0.3 & 11,6 & 0.3 \\
\hline 1-METHYL-3-ETHYLBENZENE & 116.9 & 1.0 & 2.6 & 1.4 & 26.3 & 1.1 & 2.2 & 1.2 & 41.7 & 1.1 & 43.5 & 1.2 \\
\hline 1-METHYL-4 ETHYLBENZENE & 44.3 & 0.4 & 1.1. & .0 .6 & 10.5 & 0.4 & 0.9 & 0.5 & 16.8 & 0.5 & 16.9 & 0.5 \\
\hline 1,3,5-TRIMETHYLBENZENE & 44.4 & 0.4 & 1.0 & 0.5 & 9.4 & 0.4 & 0.8 & 0.4 & 16.7 & 0.5 & 17.3 & 0.5 \\
\hline 1-METHYL-2-ETHYLBENZENE & 35.3 & 0.3 & 0.6 & 0.3 & 10.9 & 0.5 & 0.3 & .01 & $18: 3$. & 0.5 & 12.9 & 0.4 \\
\hline 1,2,4-TRIMETHYLBENZENE & 134.5 & 1.2 & 3.3 & 1.7 & 29.8 & 1.2 & 2.4 & 1.3 & 50.6 & 1.4 & 51.4 & 1.4 \\
\hline DECANE & 27. & 20.1 & 0.1 & 0.1 & 0.5 & $\mid<0.1$ & 0.1 & $\times 0.1$ & 0.9 & $<0.1$ & 0.9 & .001 \\
\hline ISOBUTYLBENZENE & 2.6 & $<0.1$ & 0.1 & 0.1 & 0.4 & $<0.1$ & 0.1 & $<0.1$ & 0.8 & $<0.1$ & 0.9 & $<0.1$ \\
\hline METHYLPROPYLBENZENE & ND & ND & ND. & No & ND & No & NB & ND & NB & NO & $\mathrm{ND}$ & ND \\
\hline S-BUTYLBENZENE & 4.1 & $<0.1$ & 0.1 & $<0.1$ & 0.8 & $<0.1$ & 0.1 & $<0.1$ & 1.3 & $<0.1$ & 1.3 & $<0.1$ \\
\hline 1-METHYL-3-ISOPROPYLBENZENE & 29.4 & 0.3 & 0.6 & 0.3 & 57 & 0.2 & 0.5 & .0 .3 & 98 & 0.3 & 10.3 & 0.3. \\
\hline 1,2,3-TRIMETHYLBENZENE & ND & ND & ND & ND & ND & ND & ND & ND & ND & ND & ND & ND \\
\hline 1 METHYL-4-ISOPROPYLBENZENE & 1.1 .1 & 2.1 & ND & $\mathrm{NB}$ & 0.2 & $2<0.1$ & NB & NB. & 0.2 & - 0.1 . & 0.3 & 20.1 \\
\hline IND AN & 13.7 & 0.1 & ND & ND & 1.9 & 0.1 & 0.2 & 0.1 & 2.7 & 0.1 & 5.3 & 0.1 \\
\hline 1. METHYL 2 ISOPROPYLBENZENE & 7.7. & 0.1 & 0.2 & 0.1 & (.). 3.6 & 0.2 & 0.2 & 0.1. & 7.0 & 02 & 1.19 & 0.1. \\
\hline 1,3-DIETHYLBENZENE & 1.1 & $<0.1$ & ND & ND & 2.7 & 0.1 & ND & ND & 0.3 & $<0.1$ & 0.3 & $<0.1$ \\
\hline 1,4-DIETHYLBENZENE & 44.8 & 0.4 & 0.4 . & 0.2 & 110 & 0.5 & 0.3 & 0.2 & 76 & 0.2. & 82 & 02 \\
\hline 1-METHYL-3-N-PROPYLBENZENE & 11.1 & 0.1 & 0.4 & 0.2 & 5.2 & 0.2 & 0.2 & 0.1 & 6.1 & 0.2 & 8.6 & 0.2 \\
\hline 1.METHYL_4-N-PROPYLBENZENE & 23.4 & 02 & 0.6 & 0.2 & 0.4 & 20.1. & 044 & 0.2 & 9.3 & 0.3 & .98 & 0.3 \\
\hline 1,2 DIETHYLBENZENE & 20.7 & 0.2 & ND & ND & 3.5 & 0.1 & 0.1 & $<0.1$ & 4.7 & 0.1 & 1.9 & 0.1 \\
\hline TMETHYLL-2-N-PROPYLBENZENE & 1.9 & $<0,1$ & $-\mathrm{NB}^{2}$ & $-\mathrm{ND}$ & 0.0 .4 & $\leq 0,1$ & .0 .1 & $80,1.1$ & 0.6 .6 & $<0.1$ & 0.3 & 30.1 \\
\hline
\end{tabular}




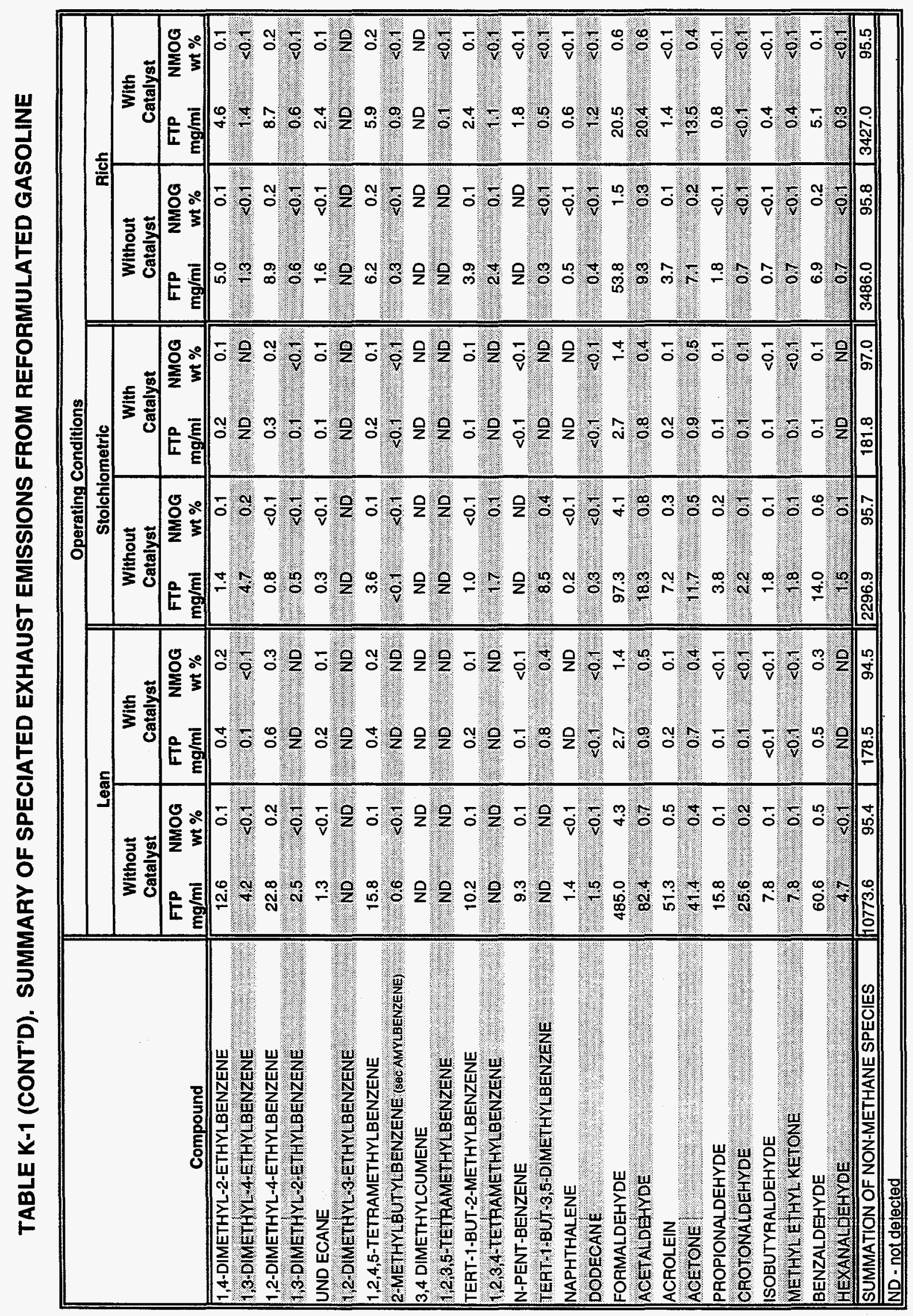


APPENDIX L

AVERAGE SPECIATED EMISSIONS RESULTS

FROM FTPS WITH ETHANOL 
TABLE L-1. SUMMARY OF SPECIATED EXHAUST EMISSIONS FROM ETHANOL

\begin{tabular}{|c|c|c|c|c|c|c|c|c|c|c|c|c|}
\hline \multirow[b]{4}{*}{ Compound } & \multicolumn{12}{|c|}{ Operating Conditions } \\
\hline & \multicolumn{4}{|c|}{ Lean } & \multicolumn{4}{|c|}{ Stoichiometric } & \multicolumn{4}{|c|}{ Rich } \\
\hline & \multicolumn{2}{|c|}{$\begin{array}{l}\text { Without } \\
\text { Catalyst }\end{array}$} & \multicolumn{2}{|c|}{$\begin{array}{c}\text { With } \\
\text { Catalyst }\end{array}$} & \multicolumn{2}{|c|}{$\begin{array}{l}\text { Without } \\
\text { Catalyst }\end{array}$} & \multicolumn{2}{|c|}{$\begin{array}{l}\text { With } \\
\text { Catalyst }\end{array}$} & \multicolumn{2}{|c|}{$\begin{array}{l}\text { Without } \\
\text { Catalyst }\end{array}$} & \multicolumn{2}{|c|}{$\begin{array}{l}\text { With } \\
\text { Catalyst }\end{array}$} \\
\hline & $\begin{array}{c}\text { FTP } \\
\mathrm{mg} / \mathrm{mi}\end{array}$ & $\begin{array}{c}\text { NMOG } \\
\text { wt \% }\end{array}$ & $\begin{array}{c}\text { FTP } \\
\mathrm{mg} / \mathrm{mi}\end{array}$ & $\begin{array}{c}\text { NMOG } \\
\text { wt \% }\end{array}$ & $\begin{array}{c}\text { FTP } \\
\mathrm{mg} / \mathrm{mi}\end{array}$ & $\begin{array}{c}\text { NMOG } \\
\text { wt \% }\end{array}$ & $\begin{array}{c}\begin{array}{c}\text { FTP } \\
\mathrm{mg} / \mathrm{mi}\end{array} \\
\end{array}$ & $\begin{array}{c}\text { NMOG } \\
\text { wt \% } \\
\end{array}$ & $\begin{array}{c}\text { FTP } \\
\mathrm{mg} / \mathrm{mi}\end{array}$ & $\begin{array}{c}\text { NMOG } \\
\text { wt \% } \\
\end{array}$ & $\begin{array}{c}\text { FTP } \\
\mathrm{mg} / \mathrm{ml}\end{array}$ & $\begin{array}{c}\text { NMOG } \\
\text { wt \% }\end{array}$ \\
\hline METHANE & 144.5 & & 54.0 & & 158.1 & & 69.9 & & 166.4 & & 121.6 & \\
\hline ETHANE & 35.6 & 0.3 & 4.9 & .066 & (1. 31. & 0.6 & 5.3 & 0.6 & 29.4 & 0.5 & 179 & 1.0 \\
\hline ETHYLENE & 455.2 & 4.2 & 29.2 & 3.0 & 310.8 & 6.0 & 28.8 & 3.1 & 279.8 & 4.9 & 32.2 & 2.8 \\
\hline PROPANE & 0.7 & $=<0.1$. & 0.3 & $1.20 .1 \%$ & 1.3 & $<0.1$. & 0.2 & $<0.1$. & 1.5 & $<0.7$ & 0.6 & 80.1 \\
\hline PROPYLENE & 3.8 & $<0.1$ & 0.6 & 0.1 & 3.4 & 0.1 & 0.7 & 0.1 & 3.0 & 0.1 & 1.4 & 0.1 \\
\hline ACETYLENE & 50.4 & 0.5 . & 15.5 & 1.6 & 708 & 1.14 & 16.5 & 18 & 80.4 & 1.4 & 18.6 & 1.6 \\
\hline PROPADIENE & ND & ND & ND & ND & ND & ND & ND & ND & ND & ND & ND & ND \\
\hline BUTANE & 0.2 & .0 .1 & 801 & 20.1 & 0.1 & .20 .1 & 50.1 & $<0.1$ & 0.2 & 20.1 & 0.11 & 20.1 \\
\hline TRANS-2-BUTENE & 0.2 & $<0.1$ & 0.1 & $<0.1$ & 0.1 & $<0.1$ & 0.1 & $<0.1$ & 0.2 & $<0.1$ & 0.2 & $<0.1$ \\
\hline TBUTENE & 0.5 & .80 .1$. & .0 .2 & $\times<0.1$. & 0.4 & $=0.4$ & 0.2 & 20.1. & 0.8 & $<01$ & 0.3 & 20.1 \\
\hline 2-METHYLPROPENE (ISOBUTYLENE) & 1.2 & $<0.1$ & 0.1 & $<0.1$ & 0.7 & $<0.1$ & 0.1 & $<0.1$ & 0.5 & $<0.1$ & 0.1 & $<0.1$ \\
\hline 2.2. DIMETHYLPROPANE (NEOPE & 20.1 & .40 .1 & $<0.1$ & .20 .1 & -20.1 & .801. & 20.1 & 201 & $<0.1$ & $\times 0.1$ & 20.1. & $<011$ \\
\hline PROPYNE & ND & ND & ND & ND & ND & ND & ND & ND & ND & ND & ND & ND \\
\hline 1,3-BUTADIENE & 1.0 & 20.1 & 0.2 & 80.1 & 0.7 & 20.1 & $0.0 .1 \%$ & $<0.1$. & 0.6 & $<0.1$ & 0.8 & 60.1 \\
\hline 2-METHYLPROPANE (ISOBUTANE) & 0.2 & $<0.1$ & 0.1 & $<0.1$ & $<0.1$ & $<0.1$ & 0.1 & $<0.1$ & ND & ND & 0.8 & 0.1 \\
\hline 1.BUTYNE & No. & ND & . ND. & . $\mathrm{ND}$ & NO & NO & . ND & NB. & ND & NB & No & ND \\
\hline METHANOL & ND & ND & ND & ND & ND & ND & ND & ND & ND & ND & ND & ND, \\
\hline CIS 2.BUTENE & 0.01 & $<0.1$ & .0 .2 & 80.1 & 0.1 & 1.0011. & 0.2 & $<0.1$ & 0.1 & 2.0 .1 & 0.1 & 80.1 \\
\hline 3-METHYL-1-BUTENE & ND & ND & ND & ND & ND & ND & ND & ND & ND & ND & ND & ND \\
\hline ETHANOL & 8799.2 & .812. & 8602 & 879 & 41110 & 79.1 & 798.4 & 85.5 & 4694.3 & 878 . & 9920 & 857 \\
\hline 2-METHYLBUTANE (ISOPENTANE) & 0.7 & $<0.1$ & 0.1 & $<0.1$ & 0.2 & $<0.1$ & 0.1 & $<0.1$ & 0.4 & $<0.1$ & 0.1 & $<0.1$ \\
\hline 2-BUTYNE & No & NO & NB & $\mathrm{ND}$ & NB & ND & $=\mathrm{NB}$ & ND.| & ND & ND: & O. & $<0.1$ \\
\hline 1-PENTENE & 0.8 & $<0.1$ & $<0.1$ & $<0.1$ & 0.2 & $<0.1$ & $<0.1$ & $<0.1$ & ND & ND & ND & ND. \\
\hline 2.METHYL 1 BUTENE & 20.1 & 20.1 & $\times 01$ & 20.1 & ND. & NB & 20.1 & 201 & 0.1 & 20.1 & 0.2 & 20.1. \\
\hline PENTANE & $<0.1$ & $<0.1$ & 0.7 & 0.1 & 0.1 & $<0.1$ & 0.2 & $<0.1$ & $<0.1$ & $<0.1$ & 0.1 & $<0.1$ \\
\hline 2.METHYL 1,3 BUTABIENE & 0.1 & .0 .1 & $\mathrm{ND}$ & NO & 04 & $<0.7$ & 20.1 & $<0.1$ & $=\mathrm{ND}$ & $1 \mathrm{NO}$ & 20.1. & 20.1 \\
\hline TRANS-2-PENTENE & 0.1 & $<0.1$ & $<0.1$ & $<0.1$ & 0.2 & $<0.1$ & $<0.1$ & $<0.1$ & 0.1 & $<0.1$ & 0.1 & $<0.1$ \\
\hline 3,3-DIMETHYL-1-BUTENE & $\mathrm{NB}$ & NB & ND & NB. & ND & ND & ND & ND & $=$ ND & $\mathrm{NO}$ & ND. & ND \\
\hline CIS-2-PENTENE & 4.3 & $<0.1$ & 0.1 & $<0.1$ & ND & ND & $<0.1$ & $<0.1$ & 0.2 & $<0.1$ & $<0.1$ & $<0.1$ \\
\hline 2.ANTHUYL-2.BIHENE & 1.0 .02 & $-20,1$ & 1.2 .0 .1$. & $=0.1$ & 201 & $=0,1$ & 01 & $=0$ & Q4. & $=81$. & O. & ant \\
\hline
\end{tabular}


TABLE L-1 (CONT'D). SUMMARY OF SPECIATED EXHAUST EMISSIONS FROM ETHANOL

\begin{tabular}{|c|c|c|c|c|c|c|c|c|c|c|c|c|}
\hline \multirow[b]{4}{*}{ Compound } & \multicolumn{12}{|c|}{ Operating Conditions } \\
\hline & \multicolumn{4}{|c|}{ Lean } & \multicolumn{4}{|c|}{ Stoichiometric } & \multicolumn{4}{|c|}{ Rich } \\
\hline & \multicolumn{2}{|c|}{$\begin{array}{l}\text { Without } \\
\text { Catalyst }\end{array}$} & \multicolumn{2}{|c|}{$\begin{array}{c}\text { With } \\
\text { Catalyst }\end{array}$} & \multicolumn{2}{|c|}{$\begin{array}{l}\text { Without } \\
\text { Catalyst }\end{array}$} & \multicolumn{2}{|c|}{$\begin{array}{c}\text { With } \\
\text { Catalyst }\end{array}$} & \multicolumn{2}{|c|}{$\begin{array}{l}\text { Without } \\
\text { Catalyst }\end{array}$} & \multicolumn{2}{|c|}{$\begin{array}{c}\text { With } \\
\text { Catalyst }\end{array}$} \\
\hline & $\begin{array}{c}\text { FTP } \\
\mathrm{mg} / \mathrm{mi}\end{array}$ & $\begin{array}{c}\text { NMOG } \\
\text { wt \% }\end{array}$ & $\begin{array}{c}\text { FTP } \\
\mathrm{mg} / \mathrm{mi}\end{array}$ & $\begin{array}{c}\text { NMOG } \\
\text { wt } \%\end{array}$ & $\begin{array}{c}\text { FTP } \\
\mathrm{mg} / \mathrm{ml}\end{array}$ & $\begin{array}{c}\text { NMOG } \\
\text { wt } \%\end{array}$ & $\begin{array}{c}\text { FTP } \\
\mathrm{mg} / \mathrm{mi}\end{array}$ & $\begin{array}{c}\text { NMOG } \\
\text { wt \% }\end{array}$ & $\begin{array}{c}\text { FTP } \\
\mathrm{mg} / \mathrm{mi}\end{array}$ & $\begin{array}{c}\text { NMOG } \\
\text { wt \% }\end{array}$ & $\begin{array}{c}\mathrm{FTP} \\
\mathrm{mg} / \mathrm{mi}\end{array}$ & $\begin{array}{c}\text { NMOG } \\
\text { wt \% }\end{array}$ \\
\hline CYCLOPENTADIENE & 0.2 & $<0.1$ & 0.1 & $<0.1$ & ND & ND & 0.1 & $<0.1$ & 0.1 & $<0.1$ & 0.1 & $<0.1$ \\
\hline 2,2 DIMETHYLBUTANE & NB & $\mathrm{ND}$ & 20.1 & $=0.1$ & 3.3 & 0.1 & .0 .01 & $<0.1$ & 0.2 & 20.1 & 0.1 & 2.20 .1 \\
\hline CYCLOPENTENE & $<0.1$ & $<0.1$ & ND & ND & 0.1 & $<0.1$ & ND & NO & $<0.1$ & $<0.1$ & $<0.1$ & $<0.1$ \\
\hline 4.METHYL-1-PENTENE & NB & NB & NO & ND & ND & $.2 . \mathrm{ND}$ & $=2.1$ & 20.1 & 0.1 & $\times 0.1$ & $\mathrm{ND}$ &. $\mathrm{ND}$ \\
\hline 3-METHYL-1-PENTENE & 0.2 & $<0.1$ & 0.1 & $<0.1$ & 0.2 & $<0.1$ & $<0.1$ & $<0.1$ & $<0.1$ & $<0.1$ & $<0.1$ & $<0.1$ \\
\hline CYCLOPENTANE & ND. & . NO.: & ND & ND & ND & NB & ND & ND. & No. & No & NB & ND \\
\hline 2,3-DIMETHYLBUTANE & 0.2 & $<0.1$ & 0.1 & $<0.1$ & 0.1 & $<0.1$ & 0.1 & $<0.1$ & 0.1 & $<0.1$ & $<0.1$ & $<0.1$ \\
\hline MTBE & 1.5 & $20.1:$ & 0.1 & $=20.1$ & 1.2 & 2.20 .1 & 0.1 & 801 & No & NO & No & NB \\
\hline 2,3-DIMETHYL-1-BUTENE & ND & ND & ND & ND & ND & ND & ND & ND & ND & ND & ND & ND \\
\hline 4-METHYLCOIS 2 PENTENE & $\%$ NO & ND & ND & $=$ ND & NO & NB & ND. & ND. & No & ND. & ND & ND. \\
\hline 2-METHYLPENTANE & 0.5 & $<0.1$ & 0.2 & $<0.1$ & 0.3 & $<0.1$ & 0.2 & $<0.1$ & 0.2 & $<0.1$ & $<0.1$ & $<0.1$ \\
\hline 4.METHYL TRANS 2-PENTENE & NO & ND & ND & ND & ND & ND & No & ND & ND & ND & ND & ND \\
\hline 3-METHYLPENTANE & 0.2 & $<0.1$ & 0.6 & 0.1 & 0.8 & $<0.1$ & 0.2 & $<0.1$ & 0.3 & $<0.1$ & 0.1 & $<0.1$ \\
\hline 2.NETHYL-I-PENTENE & 0.3 & 20.1 & 20.1 & 2.0 .1 & 0.3 & 80.1 & 01 & $<0.1$ & 0.1 & 20.1 & 20.1 & 2.20 .1 \\
\hline 1-HEXENE & 0.3 & $<0.1$ & $<0.1$ & $<0.1$ & 0.3 & $<0.1$ & 0.1 & $<0.1$ & 0.1 & $<0.1$ & $<0.1$ & $<0.1$ \\
\hline HEXANE & 0.2 & $<0.1$ & 0.1 & $<0.1$ & 0.2 & $<0.1$ & 20.1 & $<0.1$ & 0.1 & $=-0.1$ & 0.1 & 201 \\
\hline TRANS-3-HEXENE & ND & ND & ND & ND & $<0.1$ & $<0.1$ & ND & ND & $<0.1$ & $<0.1$ & $<0.1$ & $<0.1$ \\
\hline CIS 3.HEXENE & 0.1 & 20.1 & 0.1. & $=20.1$. & 0.2 & .20 .1 & $2 \times 0.1$ &.$<0.1$ & $<0.1$ & $<0.1$ & 0.01 & $<0.1$ \\
\hline TRANS-2-HEXENE & ND & ND & ND & ND & ND & ND & ND & ND & ND & ND & ND & ND \\
\hline 8-NETHYL-TRANS 2-PENTENE & ND & $.0 \mathrm{ND}$. & NE &. $\mathrm{NO}$ & $=0.1$ & 80.1 & . ND. & ND. & 0.1 & $<0.1$ & 0.1 & $(20.1$ \\
\hline 2-METHYL-2-PENTENE & ND & ND & $<0.1$ & $<0.1$ & 0.3 & $<0.1$ & ND & ND & 0.1 & $<0.1$ & ND & ND \\
\hline 3 METHYLCYCLOPENTENE & ND: & ND & ND. & . No & $\mathrm{ND}$ & ND. & NB & NO! & No & NO. & ND & NB \\
\hline CIS-2-HEXENE & 2.5 & $<0.1$ & 0.1 & $<0.1$ & ND & ND & 0.1 & $<0.1$ & 0.3 & $<0.1$ & 0.1 & $<0.1$ \\
\hline ETBE & ND. & ND & $\mathrm{ND}$ & NO & NB & ND & ND. & ND & No & ND & ND. & ND \\
\hline 3-METHYL-CIS-2-PENTENE & ND & ND & $<0.1$ & $<0.1$ & $<0.1$ & $<0.1$ & $<0.1$ & $<0.1$ & $<0.1$ & $<0.1$ & 0.2 & $<0.1$ \\
\hline 2,2-DIMETHYLPENTANE & 0.0 & 20.1. & 0.1 & $=0.1$ & 0.4 & 20.1 & 20.1 & $<0.1$ & 0.5 & 20.1 & $80 \%$ & 20.1 \\
\hline METHYLCYCLOPENTANE & ND & ND & ND & ND & ND & ND & ND & ND & ND & ND & ND & ND \\
\hline 2,4.DIMETHYLPENTANE & 0.3 & 40.1 & 0.1 & 20,1 & 0.2 & $<0.1$ & $.2001 \%$ & 20.1 & 0.1. & $<01$ & 0.1 & $<0.1$. \\
\hline 2,3,3-TRIMETHYL-1-BUTENE & ND & ND & ND & ND & ND & ND & ND & ND & ND & ND & ND & ND \\
\hline 2.2.3.TRIMETHYYLBUTANE & 0.01 & .0 .1$. & $=0.1$ & $=\leq 01$, & 3.0 .1 & $<0.4$ & $2.20,1$ & 50.1 & 1.0 .1 & 50.1 & $=.01$ & $2 \times 0.1$ \\
\hline
\end{tabular}


TABLE L-1 (CONT'D). SUMMARY OF SPECIATED EXHAUST EMISSIONS FROM ETHANOL

\begin{tabular}{|c|c|c|c|c|c|c|c|c|c|c|c|c|}
\hline \multirow[b]{4}{*}{ Compound } & \multicolumn{12}{|c|}{ Operating Conditions } \\
\hline & \multicolumn{4}{|c|}{ Lean } & \multicolumn{4}{|c|}{ Stoichiometric } & \multicolumn{4}{|c|}{ Rich } \\
\hline & \multicolumn{2}{|c|}{$\begin{array}{l}\text { Without } \\
\text { Catalyst }\end{array}$} & \multicolumn{2}{|c|}{$\begin{array}{c}\text { With } \\
\text { Catalyst }\end{array}$} & \multicolumn{2}{|c|}{$\begin{array}{l}\text { Without } \\
\text { Catalyst }\end{array}$} & \multicolumn{2}{|c|}{$\begin{array}{c}\text { With } \\
\text { Catalyst }\end{array}$} & \multicolumn{2}{|c|}{$\begin{array}{l}\text { Without } \\
\text { Catalyst }\end{array}$} & \multicolumn{2}{|c|}{$\begin{array}{c}\text { With } \\
\text { Catalyst }\end{array}$} \\
\hline & $\begin{array}{c}\text { FTP } \\
\mathrm{mg} / \mathrm{mi}\end{array}$ & $\begin{array}{c}\text { NMOG } \\
\text { wt \% } \\
\end{array}$ & $\begin{array}{c}\text { FTP } \\
\mathrm{mg} / \mathrm{mi}\end{array}$ & $\begin{array}{c}\text { NMOG } \\
\text { wt \% } \\
\end{array}$ & $\begin{array}{c}\text { FTP } \\
\mathrm{mg} / \mathrm{mi}\end{array}$ & $\begin{array}{c}\text { NMOG } \\
\text { wt \% }\end{array}$ & $\begin{array}{c}\text { FTP } \\
\mathrm{mg} / \mathrm{mi}\end{array}$ & $\begin{array}{c}\text { NMOG } \\
\text { wt \% } \\
\end{array}$ & $\begin{array}{c}\text { FTP } \\
\mathrm{mg} / \mathrm{mi}\end{array}$ & $\begin{array}{c}\text { NMOG } \\
\text { wt \% }\end{array}$ & $\begin{array}{c}\text { FTP } \\
\mathrm{mg} / \mathrm{mi}\end{array}$ & $\begin{array}{c}\text { NMOG } \\
\text { wt \% }\end{array}$ \\
\hline 3,4-DIMETHYL-1-PENTENE & ND & ND & ND & ND & ND & ND & ND & ND & ND & ND & ND & ND \\
\hline 1 METHYLCYCLOPENTENE & $<0.1$ & 20.1 & 80.1 & 201 & No & $\quad \mathrm{ND}$ & $<0.1$ & .801 & 0.4 & $20.1 \%$ & $20 \%$ & 2.00 .1 \\
\hline BENZENE & 1.8 & $<0.1$ & 0.6 & 0.1 & 1.6 & $<0.1$ & 0.7 & 0.1 & 1.6 & $<0.1$ & 1.0 & 0.1 \\
\hline 3-METHYL-1 HEXENE & ND & ND & No & No & No & No & No & ND & No & NO 1 & ND &. $\mathrm{ND}$ \\
\hline 3,3-DIMETHYLPENTANE & ND & ND & ND & ND & ND & ND & ND & ND & ND & ND & ND & ND \\
\hline OYOLOHEXANE & 0.3 & $<0.1$ & 01 & $(2,1$, & 02. & $\times 0.1$ & 0.0 .1 & 20.1 & o. & 20.1 & 201 & $\times 0.1$ \\
\hline 2-METHYLHEXANE & ND & ND & ND & ND & ND & ND & ND & ND & ND & ND & ND & ND \\
\hline 2.3. BIMETHYLPENTANE & 0.6 & $(120.1$ & 0.1 & 2.40 .1 & 0.2 & 20.1 & 0.2 & 2001 & 0.2 & 20.1. & 11 & 0.1 \\
\hline 1,1-DIMETHYLCYCLOPENTANE & ND & ND & ND & ND & ND & ND & ND & ND & ND & ND & ND & ND \\
\hline CYCLOHEXENE & ND & ND & NB & $=\mathrm{NB}$ & ND & NB & ND. & NO & NB & No & No & $1 \mathrm{NO}$ \\
\hline 3-METHYLHEXANE & 0.2 & $<0.1$ & $<0.1$ & $<0.1$ & 0.1 & $<0.1$ & $<0.1$ & $<0.1$ & 0.1 & $<0.1$ & 2.3 & 0.2 \\
\hline CIS -1 3.BIMETHYLCYCLOPENTA & ND. & $1.9 \mathrm{NO}$ & No & ND & 0.1 & 2.8 .1$. & No & NO & .80 .1 & $<0.1 \%$ & $\mathrm{ND}$ & ND \\
\hline 3-ETHYLPENTANE & ND & ND & ND & ND & ND & ND & ND & ND & ND & ND & ND & ND \\
\hline TRANS 1,2 DIMETHYLCYCLOPENTANE & ND & ND & ND & No & NO & ND & ND & ND. & ND & ND & $\mathrm{ND}$ & ND \\
\hline TRANS-1,3-DIMETHYLCYCLOPENTANE & ND & ND & ND & ND & ND & ND & ND & ND & ND & NO & ND & ND \\
\hline 1HEPTENE & ND: & NO & ND & ND. & ND & NB. & NB & NG & $1 . N B$ & ND & NDE & ND \\
\hline 2,2,4-TRIMETHYLPENTANE & 1.8 & $<0.1$ & 0.2 & $<0.1$ & 0.7 & $<0.1$ & 0.4 & $<0.1$ & 0.7 & $<0.1$ & 0.2 & $<0.1$ \\
\hline 2.METHYL 1 HEXENE & ND & NB. & NO & ND & No & No & No. & ND & No & No & ND: & ND \\
\hline TRANS-3-HEPTENE & ND & ND & ND & ND & ND & ND & ND & ND & ND & ND & ND & ND \\
\hline HEPTANE & 02 & $<0.1:$ & 0.1 & 201 & 0.1 & $=<0.1$ & $+<01$ & 20.1. & 0.1 & 20.1 & 0.1 & 20.1 \\
\hline 2-METHYL-2-HEXENE & ND & ND & ND & ND & ND & ND & ND & ND & ND & ND & $<0.1$ & $<0.1$ \\
\hline 3-METHYL-TRANS - 3 HEXENE & ND & ND: & ND. & ND & ND & ND? & ND & NB & ND. & $\mathrm{NB}$ & $\mathrm{ND}$ & ND: \\
\hline TRANS-2-HEPTENE & ND & ND & ND & ND & ND & ND & ND & ND & ND & ND & ND: & ND \\
\hline 3-ETHVL-CIS-2-PENTENE & ND & ND $:$ & ND & ND & NB & ND & NO & No. & NO & NO & ND & No \\
\hline 2,4,4-TRIMETHYL-1-PENTENE & ND & ND & ND & ND & ND & ND & ND & ND & ND & ND & $<0.1$ & $<0.1$ \\
\hline 2,2,4 TRIMETHYL-T PENTENE & $\mathrm{NB}$ & NO & ND. & No & ND &. $\mathrm{NB}$ & ND & No & No & $\mathrm{ND}$ & ND & $\mathrm{ND}$ \\
\hline 2,3-DIMETHYL-2-PENTENE & ND & ND & ND & ND & ND & ND & ND & ND & ND & ND & ND & ND \\
\hline CIS-HEPTENE & NO. & NB & Ne. & No & ND & ND. & ND & NO & NB & (2. NB & ND & ND \\
\hline METHYLCYCLOHEXANE & ND & ND & ND & ND & ND & ND & ND & ND & ND & ND & ND & ND \\
\hline GS 1.2DIMETHYKCYCLOPENTANE. & L $\mathrm{ND}$ & $=\mathrm{ND}$ & $2 . \mathrm{ND}$ & $\mathrm{ND} \cdot 1$ & $1 . \mathrm{NB}$ & No. & N.NO & No. & ND & No. 1 & $1 . \mathrm{NO}$ & ND. \\
\hline
\end{tabular}


TABLE L-1 (CONT'D). SUMMARY OF SPECIATED EXHAUST EMISSIONS FROM ETHANOL

\begin{tabular}{|c|c|c|c|c|c|c|c|c|c|c|c|c|}
\hline \multirow[b]{4}{*}{ Compound } & \multicolumn{12}{|c|}{ Operating Conditions } \\
\hline & \multicolumn{4}{|c|}{ Lean } & \multicolumn{4}{|c|}{ Stoichiometric } & \multicolumn{4}{|c|}{ Rich } \\
\hline & \multicolumn{2}{|c|}{$\begin{array}{l}\text { Without } \\
\text { Catalyst }\end{array}$} & \multicolumn{2}{|c|}{$\begin{array}{c}\text { With } \\
\text { Catalyst }\end{array}$} & \multicolumn{2}{|c|}{$\begin{array}{l}\text { Without } \\
\text { Catalyst }\end{array}$} & \multicolumn{2}{|c|}{$\begin{array}{c}\text { With } \\
\text { Catalyst }\end{array}$} & \multicolumn{2}{|c|}{$\begin{array}{l}\text { Without } \\
\text { Catalyst }\end{array}$} & \multicolumn{2}{|c|}{$\begin{array}{c}\text { With } \\
\text { Catalyst }\end{array}$} \\
\hline & $\begin{array}{c}\text { FTP } \\
\mathrm{mg} / \mathrm{mi}\end{array}$ & $\begin{array}{c}\text { NMOG } \\
\text { wt \% }\end{array}$ & $\begin{array}{c}\text { FTP } \\
\mathrm{mg} / \mathrm{mi}\end{array}$ & $\begin{array}{c}\text { NMOG } \\
\text { wt \% }\end{array}$ & $\begin{array}{c}\text { FTP } \\
\mathrm{mg} / \mathrm{mi}\end{array}$ & $\begin{array}{c}\text { NMOG } \\
\text { wt \% } \\
\end{array}$ & $\begin{array}{c}\text { FTP } \\
\mathrm{mg} / \mathrm{mi}\end{array}$ & $\begin{array}{c}\text { NMOG } \\
\text { wt } \% \\
\end{array}$ & $\begin{array}{c}\text { FTP } \\
\mathrm{mg} / \mathrm{ml}\end{array}$ & $\begin{array}{c}\text { NMOG } \\
\text { wt \% }\end{array}$ & $\begin{array}{c}\text { FTP } \\
\mathrm{mg} / \mathrm{mi}\end{array}$ & $\begin{array}{c}\text { NMOG } \\
\text { wt \% } \\
\end{array}$ \\
\hline 2,2-DIMETHYLHEXANE & 0.3 & $<0.1$ & 0.1 & $<0.1$ & ND & ND & $<0.1$ & $<0.1$ & 0.2 & $<0.1$ & $<0.1$ & $<0.1$ \\
\hline 1,1,3-TRIMETHYLCYCLOPENTANE & $\mathrm{ND}$ & ND & ND & ND & ND & ND & ND & NO & NB & ND & ND & ND \\
\hline 2,4,4-TRIMETHYL-2-PENTENE & ND & ND & $<0.1$ & $<0.1$ & ND & ND & ND & ND & ND & ND & ND & ND \\
\hline 2.2,3-TRIMETHYLPENTANE & ND & ND & No & ND & ND & ND & ND & 1. $\mathrm{ND}$ & ND. & NB & ND & ND \\
\hline 2,5-DIMETHYLHEXANE & ND & ND & ND & ND & ND & ND & ND & ND & ND & ND & ND & ND \\
\hline ETHYLCYCLOPENTANE & ND & ND. & ND & ND: & ND & NB & NB. & No & ND & $\mathrm{ND}$ & ND & ND: \\
\hline 2,4-DIMETHYLHEXANE & 0.3 & $<0.1$ & $<0.1$ & $<0.1$ & ND & ND & $<0.1$ & $<0.1$ & $<0.1$ & $<0.1$ & $<0.1$ & $<0.1$ \\
\hline 1-TRANS 2-CIS 4-TRIMETHYLCYCLOPENTANE & No. & ND & ND & ND & NO & NB & ND & ND. & $\mathrm{ND}$ & No & No & NO \\
\hline 3,3-DIMETHYLHEXANE & ND & ND & ND & ND & ND & ND & ND & ND & ND & ND & ND & ND \\
\hline 1-TRANS-2-OIS-3-TRIMETHYLCYCLOPENTANE: & ND & ND & ND & ND & ND & ND & $\mathrm{NO}$ & ND & ND & NO & No & No \\
\hline 2,3,4-TRIMETHYLPENTANE & ND & ND & ND & ND & ND & ND & ND. & ND & ND & ND & ND & ND \\
\hline 2,3,3 TRIMETHYLPENTANE & 0.3 & 2.0 .1 & 0.1 & $<0.1$ & 0.1 & 20.1 & $<0.1$ & 20.1 & 0.3 & 20.1 & 0.1 & <0.1. \\
\hline TOLUENE & 2.3 & $<0.1$ & 0.5 & $<0.1$ & 1.0 & $<0.1$ & 0.5 & 0.1 & 1.7 & $<0.1$ & 0.8 & 0.1 \\
\hline 2,3-DIMETHYLHEXANE: & 0.4 & 20.1. & ND &. ND & 0.1 & $=20.1$ & ND. & ND & 0.1 & 2.80 .1 & 0.1 & 1.20 .1 \\
\hline 1,1,2-TRIMETHYLCYCLOPENTANE & ND & ND & ND & ND & ND & ND & ND & ND & ND & ND & ND & ND \\
\hline (2METHYLHEPTANE & ND &. $\mathrm{ND}$. & ND & ND & ND & ND & No & ND & ND & ND & 80.1 & $<0.1$ \\
\hline 3,4-DIMETHYLHEXANE & ND & ND & ND & ND & ND & ND & ND & ND & ND & ND & ND & ND \\
\hline 2,2,4,4-TETRAMETHYLPENTANE & ND. & ND. & No & ND & ND & ND & ND. & ND & $\mathrm{NB}$ & $\mathrm{No}$ & ND & ND. \\
\hline 4-METHYLHEPTANE & ND & ND & ND & ND & ND & ND & ND & ND & ND & ND & ND & ND \\
\hline 2. METHYL-3-ETHYLPENTANE & ND & NO & ND & NO & ND & ND & NB & ND & NO & ND: & ND & NB \\
\hline 2,6-DIMETHYLHEPTANE & ND & ND & ND & ND & ND & ND & ND & ND & ND & ND & ND & ND \\
\hline 3METHYLHEPTANE & ND & ND. & ND & ND & NO: & ND. & . ND. & ND & NO & No. & 20.1 & 20.1 \\
\hline 1-CIS,2-TRANS,3-TRIMETHYLCYCLOPENTANE & ND & ND & ND & ND & ND & ND & ND & ND & ND & ND & ND & ND \\
\hline CIS-1, 3-DINETHYLCYCLOHEXANE & ND & NB & ND & No & ND & ND & ND: & ND & ND. & ND & ND. & ND \\
\hline TRANS-1,4-DIMETHYLCYCLOHEXANE & ND & ND & ND & ND & ND & ND & ND & ND & $<0.1$ & $<0.1$ & $<0.1$ & $<0.1$ \\
\hline 3-ETHYLHEXANE & $\mathrm{NB}$ & $\mathrm{NB}$ & ND & ND & ND & No & ND & NB. & NB & No & ND & No \\
\hline 2,2,5-TRIMETHYLHEXANE & ND & ND & ND & ND & ND & ND & ND & ND & ND & ND & $<0.1$ & $<0.1$ \\
\hline CIS-1 METHYL-3-ETHYLCYCLOPENTANE & ND: & ND & NO & No & ND & NO & No & No & $\mathrm{ND}$ & ND: & ND & No \\
\hline 1,1-DIMETHYLCYCLOHEXANE & ND & ND & ND & ND & ND & ND & ND & ND & ND & ND & ND & ND \\
\hline TRANS 1-METHYL-2ETHYLCYCLO & $\mathrm{NB}$ & $\mathrm{NB}$ & $=\mathrm{ND}$ & ND & $\mathrm{ND}$ & ND & NO & ND & NB & ND & $=\mathrm{ND}$ & No \\
\hline
\end{tabular}


TABLE L-1 (CONT'D). SUMMARY OF SPECIATED EXHAUST EMISSIONS FROM ETHANOL.

\begin{tabular}{|c|c|c|c|c|c|c|c|c|c|c|c|c|}
\hline \multirow[b]{4}{*}{ Compound } & \multicolumn{12}{|c|}{ Operating Conditions } \\
\hline & \multicolumn{4}{|c|}{ Lean } & \multicolumn{4}{|c|}{ Stoichlometric } & \multicolumn{4}{|c|}{ Rich } \\
\hline & \multicolumn{2}{|c|}{$\begin{array}{l}\text { Without } \\
\text { Catalyst }\end{array}$} & \multicolumn{2}{|c|}{$\begin{array}{c}\text { With } \\
\text { Catalyst }\end{array}$} & \multicolumn{2}{|c|}{$\begin{array}{l}\text { Without } \\
\text { Catalyst }\end{array}$} & \multicolumn{2}{|c|}{$\begin{array}{c}\text { With } \\
\text { Catalyst }\end{array}$} & \multicolumn{2}{|c|}{$\begin{array}{l}\text { Without } \\
\text { Catalyst }\end{array}$} & \multicolumn{2}{|c|}{$\begin{array}{c}\text { With } \\
\text { Catalyst }\end{array}$} \\
\hline & $\begin{array}{c}\text { FTP } \\
\mathrm{mg} / \mathrm{mi}\end{array}$ & $\begin{array}{c}\text { NMOG } \\
\text { wt \% }\end{array}$ & $\begin{array}{c}\text { FTP } \\
\mathrm{mg} / \mathrm{mi}\end{array}$ & $\begin{array}{c}\text { NMOG } \\
\text { wt \% }\end{array}$ & $\begin{array}{c}\text { FTP } \\
\mathrm{mg} / \mathrm{mi}\end{array}$ & $\begin{array}{c}\text { NMOG } \\
\text { wt \% }\end{array}$ & $\begin{array}{c}\text { FTP } \\
\mathrm{mg} / \mathrm{mi}\end{array}$ & $\begin{array}{c}\text { NMOG } \\
\text { wt \% }\end{array}$ & $\begin{array}{c}\text { FTP } \\
\mathrm{mg} / \mathrm{mi}\end{array}$ & $\begin{array}{c}\text { NMOG } \\
\text { wt \% }\end{array}$ & $\begin{array}{c}\text { FTP } \\
\mathrm{mg} / \mathrm{mi}\end{array}$ & $\begin{array}{l}\text { NMOG } \\
\text { wt \% }\end{array}$ \\
\hline 1-METHYL-1-ETHYL-CYCLOPENTANE & ND & ND & ND & ND & ND & ND & ND & ND & ND & ND & $\overline{\mathrm{ND}}$ & ND \\
\hline 2,4,4TRIMETHYLLEXANE & NB. & NB & $\mathrm{NB}$ & ND & ND & No & ND & (2. $\mathrm{ND}$ & NB & ND & No & No \\
\hline 2,2,4-TRIMETHYLHEXANE & ND & ND & ND & ND & ND & ND & ND & ND & ND & ND & ND & ND \\
\hline TRANS-1, 2-DIMETHYLCYCLOHEXANE & ND & ND & $\mathrm{ND}$ & No & No & ND. & ND & ND & ND & (2. NB. & $\mathrm{NO}$ & No \\
\hline 1.OCTENE & ND & ND & ND & ND & ND & ND & ND & ND & ND & ND & ND & ND \\
\hline TAANS 4 -OCTENE & NB & No & ND. & NB & ND & ND & NB. & ND & NB & NB & $\mathrm{ND}$ & $\mathrm{NB}$ \\
\hline OCTANE & ND & ND & ND & ND & $<0.1$ & $<0.1$ & ND & ND & ND & ND & 0.2 & $<0.1$ \\
\hline TRANS-2-OOTENE & ND & ND & NB & ND. & ND. & NB & ND & $.2 \mathrm{ND}$ & $\mathrm{ND}$ & No & ND & NO \\
\hline TRANS-1,3-DIMETHYLCYCLOHEXANE & ND & ND & NO & ND & ND & ND & ND & ND & ND & ND & ND & ND \\
\hline CIS 1 4 DIMETHYLOYCLOHEXANE & NO & (1) $\mathrm{NB}$ & 1. $\mathrm{NB}$. & NO & NB & . NO & No & ND. & NB & ND. & $\mathrm{ND}$ & No \\
\hline CIS-2-OCTENE & ND & ND & ND & ND & ND & ND & ND & ND & ND & ND & ND & ND \\
\hline 2,3,6-TRIMETHYLHEXANE & ND. & (.) NB & ND. & ND & . NO & No. & $1 . \mathrm{NO}$ & $\mathrm{NB}$ & ND & ND & $\mathrm{ND}$ & No \\
\hline CIS-1-METHYL-2-ETHYLCYCLOPENTANE & ND & ND & ND & NO & ND & ND & ND & ND & ND. & ND & ND & ND \\
\hline 2-NETHYL-2-ETHYLHEPTANE & NO & $\mathrm{NB}$ & No. & No & ND. & $\mathrm{ND}$ & ND. & ND & NB. & NB. & ND. & ND. \\
\hline 2,4-DIMETHYLHEPTANE & 0.6 & $<0.1$ & 1.4 & 0.1 & 1.0 & $<0.1$ & 1.2 & 0.1 & 0.3 & $<0.1$ & 0.8 & 0.1 \\
\hline 4.4-DIMETHYLHEPTANE & $1 . \mathrm{ND}$. & ND & ND. & ND. & No & $\mathrm{NB}$ & No & No & No & ND & ND & NO \\
\hline CIS-1,2-DIMETHYLCYCLOHEXANE & ND & ND & ND & ND & ND & ND & ND & ND & ND & ND & 0.1 & $<0.1$ \\
\hline ETHYLCYOLOHEXANE & No & $\mathrm{ND}$ & ND. & ND & ND & ND. & NO & No & ND. & $\mathrm{NO}$ & No. & No \\
\hline PROPYLCYCLOHEXANE & ND & ND & ND & ND & ND & ND & ND & ND & ND & ND & ND & ND \\
\hline 2.METHYL 4-ETHYLHEXANE & . ND. & ND & ND & $\mathrm{ND}$ & ND & ND & ND & ND & ND & NB & ND. & ND. \\
\hline 2,6-DIMETHYLHEPTANE & ND & ND & ND & ND & ND & ND & ND & ND & ND & ND & ND & ND \\
\hline 11,3-TAIMETHYLO COLOHEXANE & ND & NB & ND & $\mathrm{ND}$ & $\mathrm{ND}$. & ND & . NO & $\mathrm{NB}$ & NB. & ND & $\mathrm{ND}$ & No \\
\hline 2,5-DIMETHYLHEPTANE & ND & ND & ND & ND & ND & ND & ND & ND & ND & ND & ND & ND \\
\hline 3,3-DIMETHYLHEPTANE. & ND. & ND. & ND & NB & ND & $\mathrm{ND}$ & $1.2 . \mathrm{ND}$ & ND. & $\mathrm{NB}$ & No: & ND. & ND \\
\hline 3,5-DIMETHYLHEPTANE & ND & ND & ND & ND & ND & ND & ND & ND & ND & ND. & ND & ND \\
\hline ETHYLBENZENE & 0.4 & 20.1. & 0.1 & $<01 \%$ & 0.3 & 20.1 & 0.1 & 20.1 & 0.2 & $80.1:$ & 0.1 & 20,1 \\
\hline 2,3-DIMETHYLHEPTANE & ND & ND & ND & ND & ND & ND & ND & ND & ND & ND & ND & ND \\
\hline M- \& P XYLENE & 1.0 .0 & $<0.1$ & 0.1 & 801 & 0.5 & 20.1 & 0.2 & 20.1 & 03 & 80.1. & 0.3 & $\times 0.1$ \\
\hline 4-METHYLOCTANE & ND & ND & ND & ND & ND & ND & ND & ND & ND & ND & ND & ND \\
\hline 2METHYYOOTANE & ND & $\mathrm{ND}$ & NO & ND & 0.1 & 80.1 & $\mathrm{ND}$ & ND & NB & ND & 50.1 & 80.1 \\
\hline
\end{tabular}


TABLE L-1 (CONT'D). SUMMARY OF SPECIATED EXHAUST EMISSIONS FROM ETHANOL

\begin{tabular}{|c|c|c|c|c|c|c|c|c|c|c|c|c|}
\hline \multirow[b]{4}{*}{ Compound } & \multicolumn{12}{|c|}{ Operating Conditions } \\
\hline & \multicolumn{4}{|c|}{ Lean } & \multicolumn{4}{|c|}{ Stoichlometric } & \multicolumn{4}{|c|}{ Rich } \\
\hline & \multicolumn{2}{|c|}{$\begin{array}{l}\text { Without } \\
\text { Catalyst }\end{array}$} & \multicolumn{2}{|c|}{$\begin{array}{c}\text { With } \\
\text { Catalyst }\end{array}$} & \multicolumn{2}{|c|}{$\begin{array}{l}\text { Without } \\
\text { Catalyst }\end{array}$} & \multicolumn{2}{|c|}{$\begin{array}{l}\text { With } \\
\text { Catalyst }\end{array}$} & \multicolumn{2}{|c|}{$\begin{array}{l}\text { Without } \\
\text { Catalyst }\end{array}$} & \multicolumn{2}{|c|}{$\begin{array}{c}\text { With } \\
\text { Catalyst }\end{array}$} \\
\hline & \begin{tabular}{|c|} 
FTP \\
$\mathrm{mg} / \mathrm{ml}$
\end{tabular} & $\begin{array}{c}\text { NMOG } \\
\text { wt \% }\end{array}$ & $\begin{array}{c}\text { FTP } \\
\mathrm{mg} / \mathrm{mi}\end{array}$ & $\begin{array}{c}\text { NMOG } \\
\text { wt \% } \\
\end{array}$ & $\begin{array}{c}\text { FTP } \\
\mathrm{mg} / \mathrm{mi}\end{array}$ & $\begin{array}{c}\text { NMOG } \\
w t \% \\
\end{array}$ & $\begin{array}{c}\text { FTP } \\
\mathrm{mg} / \mathrm{mi}\end{array}$ & $\begin{array}{c}\text { NMOG } \\
\text { wt } \% \\
\end{array}$ & $\begin{array}{c}\text { FTP } \\
\mathrm{mg} / \mathrm{ml}\end{array}$ & $\begin{array}{c}\text { NMOG } \\
\text { wt \% } \\
\end{array}$ & $\begin{array}{c}\mathrm{FTP} \\
\mathrm{mg} / \mathrm{mi}\end{array}$ & $\begin{array}{c}\text { NMOG } \\
\text { wt \% } \\
\end{array}$ \\
\hline 3-METHYLOCTANE & ND & ND & ND & ND & ND & ND & ND & ND & ND & ND & 0.2 & $<0.1$ \\
\hline STYRENE & NB & NB & ND & ND & ND & ND. & ND & ND & NB & ND & ND & No \\
\hline O-XYLENE & 0.3 & $<0.1$ & 0.1 & $<0.1$ & 0.2 & $<0.1$ & 0.1 & $<0.1$ & 0.1 & $<0.1$ & 0.2 & $<0.1$ \\
\hline 2.4.6-TRIMETHYLHEXANE & ND. & . $\mathrm{ND}$ & No & ND & No & ND: & ND & ND & ND & ND & $=\mathrm{NO}$ & NB: \\
\hline 1-NONENE & ND & ND & ND & ND & ND & ND & ND & ND & ND & ND & ND & ND \\
\hline NONANE & ND: & ND & ND & NO : & ND & ND. & ND & ND & NO & ND & ND & ND. \\
\hline ISOPROPYLBENZENE (CUMENE) & ND & ND & ND & ND & ND & ND & ND & ND & ND & ND & $<0.1$ & $<0.1$ \\
\hline 2.2.DIMETHYLOCTANE. & NO & ND & No & ND. & ND & NB. & ND & ND. & ND & NB. & 0.1 & 20. \\
\hline 2,4-DIMETHYLOCTANE & ND & ND & ND & ND & ND & ND & $<0.1$ & $<0.1$ & ND & ND & 0.1 & $<0.1$ \\
\hline I.PROPYLBENZENE & NG & ND & ND & ND. & No & NO & ND & ND & $<0.1$ & $\times 0.1$ & 80.1 & $<0.1$ \\
\hline 1-METHYL-3-ETHYLBENZENE & 0.3 & $<0.1$ & 0.1 & $<0.1$ & 0.2 & $<0.1$ & 0.1 & $<0.1$ & 0.3 & $<0.1$ & 0.1 & $<0.1$ \\
\hline 1.METHYL-4-ETHMLBENZENE & 0.1 & 50.1 & 20.1 & $.201 \%$ & $\mathrm{NO}$ & No & 8.20. & 20.1 & 0.1 & $<0.1$ & 0.2 & 80.1 \\
\hline 1,3,5-TRIMETHYLBENZENE & 0.1 & $<0.1$ & ND & ND & ND & ND & $<0.1$ & $<0.1$ & 0.2 & $<0.1$ & 0.4 & $<0.1$ \\
\hline 1.METHYL-2-ETHYLBENZENE & .0 .1$. & $.80 .1 \%$ & $.0 \mathrm{NB}$ & NO & 20.1 & 2.0 .1 & 0.1 & $20.1 \%$ & 0.1 & 2.0 .1 & 0.1 & 20.1 \\
\hline 1,2,4-TRIMETHYLBENZENE & 0.5 & $<0.1$ & 0.2 & $<0.1$ & 0.1 & $<0.1$ & 0.1 & $<0.1$ & 0.3 & $<0.1$ & 0.2 & $<0.1$ \\
\hline DECANE & 0.1 & $<0.1$ & 0.2 & $<0.1$ & 0.2 & .20 .1 & 0.1. & $<01$ & 0.1 & 20.1 & 0.1 & $<0.1$ \\
\hline ISOBUTYLBENZENE & 0.1 & $<0.1$ & 0.2 & $<0.1$ & 0.2 & $<0.1$ & 0.1 & $<0.1$ & 0.1 & $<0.1$ & 0.1 & $<0.1$ \\
\hline METHYLPROPYLBENZENE & ND. & $.2 . \mathrm{ND}$ & No & ND. & ND & ND. & ND & ND & No. & NB & $\mathrm{NO}$ & ND \\
\hline S-BUTYLBENZENE & ND & ND & ND & ND & ND & ND & ND & ND & ND & ND & ND & ND \\
\hline 1-METHYL-3 ISOPROPYLBENZENE & 0.1 & .0 .1 & No & $1.2 \mathrm{NB}$ & $\mathrm{NB}$ & . $\mathrm{NB}$ & 20.1 & .80 .1 & $<0 \%$ & 80.1 & 2001 & .0 .1 \\
\hline 1,2,3-TRIMETHYLBENZENE & ND & ND & ND & ND & ND & ND & ND & ND & ND & ND & ND & ND \\
\hline 1.METHYL-4-ISOPROPYLBENZENE & NO & $\mathrm{NB}$ & ND & ND? & No & 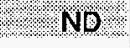 & NB & ND & 201 & $<0.1$ & ND & ND \\
\hline IND AN & ND & ND & ND & ND & ND & ND & ND & ND & ND & ND & $<0.1$ & $<0.1$ \\
\hline 1.METHYL-2 ISOPROPYLBENZENE & 801 & 80.1 & ND & ND & No: & $.2 \mathrm{NB}$ & No: & ND & 2.1. & 20.1 & 20.1 & $<0.1$ \\
\hline 1,3-DIETHYLBENZENE & ND & ND & ND & ND & ND & ND & ND & ND & ND & ND & ND & ND \\
\hline 1,4 DIETHYLBENZENE & ND. & ND & ND & ND & ND & ND & $\mathrm{ND}$ & ND. & ND & $\mathrm{ND}$ & 0.2 & $<0.1$ \\
\hline 1-METHYL-3-N-PROPYLBENZENE & ND & ND & ND & ND & ND & ND & ND & ND & ND & ND & ND & ND \\
\hline 1.METHYL-4-N-PROPYLBENZENE & ND. & ND & ND & NO & No & $\mathrm{NB}$ & ND & ND & no. & $\mathrm{NO}$ & NO & ND \\
\hline 1,2 DIETHYLBENZENE & ND & ND & ND & ND & ND & ND & ND & ND & ND & ND & ND & ND. \\
\hline 1.METHYL-2-N-PROPYLBENZENE & $\mathrm{ND}$ & ND. & $3 . \mathrm{NB}$ & ND & ND & ND & $1 . \mathrm{NB}$ & $\mathrm{ND}$ & NO & ND & ND & $\mathrm{NB}$ \\
\hline
\end{tabular}


TABLE L-1 (CONT'D). SUMMARY OF SPECIATED EXHAUST EMISSIONS FROM ETHANOL

\begin{tabular}{|c|c|c|c|c|c|c|c|c|c|c|c|c|}
\hline \multirow[b]{4}{*}{ Compound } & \multicolumn{12}{|c|}{ Operating Conditions } \\
\hline & \multicolumn{4}{|c|}{ Lean } & \multicolumn{4}{|c|}{ Stoichiometrle } & \multicolumn{4}{|c|}{ Rich } \\
\hline & \multicolumn{2}{|c|}{$\begin{array}{l}\text { Without } \\
\text { Catalyst }\end{array}$} & \multicolumn{2}{|c|}{$\begin{array}{c}\text { With } \\
\text { Catalyst }\end{array}$} & \multicolumn{2}{|c|}{$\begin{array}{l}\text { Without } \\
\text { Catalyst }\end{array}$} & \multicolumn{2}{|c|}{$\begin{array}{c}\text { With } \\
\text { Catalyst }\end{array}$} & \multicolumn{2}{|c|}{$\begin{array}{l}\text { Without } \\
\text { Catalyst }\end{array}$} & \multicolumn{2}{|c|}{$\begin{array}{c}\text { With } \\
\text { Catalyst }\end{array}$} \\
\hline & $\begin{array}{c}\text { FTP } \\
\mathrm{mg} / \mathrm{mi}\end{array}$ & $\begin{array}{c}\text { NMOG } \\
\text { wt \% }\end{array}$ & $\begin{array}{c}\mathrm{FTP} \\
\mathrm{mg} / \mathrm{mi}\end{array}$ & $\begin{array}{c}\text { NMOG } \\
\text { wt \% }\end{array}$ & $\begin{array}{c}\mathrm{FTP} \\
\mathrm{mg} / \mathrm{mi}\end{array}$ & $\begin{array}{c}\text { NMOG } \\
\text { wt \% }\end{array}$ & $\begin{array}{c}\text { FTP } \\
\mathrm{mg} / \mathrm{mi}\end{array}$ & $\begin{array}{c}\text { NMOG } \\
\text { wt \% }\end{array}$ & $\begin{array}{c}\text { FTP } \\
\mathrm{mg} / \mathrm{mi}\end{array}$ & $\begin{array}{c}\text { NMOG } \\
\text { wt \% }\end{array}$ & $\begin{array}{c}\text { FTP } \\
\mathrm{mg} / \mathrm{mi}\end{array}$ & $\begin{array}{l}\text { NMOG } \\
\text { wt \% }\end{array}$ \\
\hline 1,4-DIMETHYL-2-ETHYLBENZENE & ND & ND & ND & ND & ND & ND & $\overline{N D}$ & ND & ND & ND & ND & ND \\
\hline 1.3.DIMETHYL-4-ETHYLBENZENE & NO & NO & No & ND & ND & NO & ND. & $\mathrm{NO}$ & NO & ND & No & ND \\
\hline 1,2-DIMETHYL-4-ETHYLBENZENE & ND & ND & ND & ND & ND & ND & ND & ND & ND & ND & $<0.1$ & $<0.1$ \\
\hline 1,3-DIMETHYL-2-ETHYLBENZENE & ND & ND & ND: & ND. & ND & 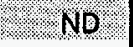 & ND. & No & ND & NB & $1.2 . \mathrm{ND}$ & . $\mathrm{ND}$ \\
\hline UND ECANE & ND & ND & $<0.1$ & $<0.1$ & No & ND & ND & ND & ND & ND & $<0.1$ & $<0.1$ \\
\hline 1,2.DIMETHYL-3-ETHYLBENZENE & ND. & ND & ND. & . ND & ND & No $:$ & $1.1 . \mathrm{ND}$ & $\mathrm{ND}$ & ND & ND. & $<0.1$ & $=0.1$ \\
\hline 1,2,4,5-TETRAMETHYLBENZENE & ND & ND & ND & ND & ND & ND & ND & ND & ND & ND & ND & ND \\
\hline 2.METHYLBUT YLBENZENE ISBO AMYLBENZENE & ND & ND & ND. & ND & No & \%. NB. & 1. ND. & ND & No & . ND. & No & NB \\
\hline 3,4 DIMETHYLCUMENE & ND & ND & ND & ND & ND & ND & ND & ND & ND & ND & ND & ND \\
\hline $1,2,3,5-T E T R A M E T H Y L B E N Z E N E$ & ND. & ND & NO. & ND &. $\mathrm{NB}$ & NDB & $1.2 \mathrm{NO}$ & ND. & ND & $\mathrm{NO}$ & $\mathrm{NO}$ & ND \\
\hline TERT-1-BUT-2-METHYLBENZENE & ND & ND & ND & ND & ND & ND & ND & ND & ND & ND & $<0.1$ & $<0.1$ \\
\hline 1.2,6,4TETRAMETHYYLBENZENE & NB & ND. & ND & ND & ND. ND & ND & ND & ND. & ND & ND & $\mathrm{ND}$ & $\mathrm{NB}$ \\
\hline N-PENT-BENZENE & 0.2 & $<0.1$ & 0.3 & $<0.1$ & 0.1 & $<0.1$ & 0.2 & $<0.1$ & ND & ND & ND & ND \\
\hline TERT-1 BUT -3,5-DIMETHYLBENZENE & ND & ND & N: ND: & ND & I. $\mathrm{NB}$ & ND & ND & $\mathrm{ND}$ & ND & $\mathrm{NB}$ & $1.2 \mathrm{ND}$ & ND \\
\hline NAPHTHALENE & ND & ND & ND & ND & ND & ND & ND & ND & ND. & ND & ND & ND \\
\hline DOBECANE & NO & ND & ND. & ND & $\because \mathrm{ND}$ & No & . ND. & NO & NB & ND. & 0.1 & 20.1 \\
\hline FORMALDEHYDE & 318.2 & 2.9 & 6.1 & 0.6 & 166.1 & 3.2 & 5.3 & 0.6 & 172.4 & 3.0 & 7.2 & 0.6 \\
\hline ACETALDEHYDE & 1131.2 & 104. & 525 & 5.4 & 478.1 & 9.2 & 66.2 & 71 & 458.4 & 8.0 & 63.4 & 5.5 \\
\hline ACROLEIN & 10.5 & 0.1 & 0.4 & $<0.1$ & 5.8 & 0.1 & 0.6 & 0.1 & 3.1 & 0.1 & 0.4 & $<0.1$ \\
\hline ACETONE & 0.1 & $=20.1$ & 02 & 201 & 0.2 & 201 & 2.8 & 0.8 & .044 & $\times 0.1$ & 9.9 & 0.9 \\
\hline PROPIONALDEHYDE & 1.6 & $<0.1$ & 0.2 & $<0.1$ & 0.6 & $<0.1$ & 0.9 & 0.1 & 0.9 & $<0.1$ & 0.8 & 0.1 \\
\hline CROTONALDEHYDE & 0.2 & 80.1 & 0.3 & $<0.1$ & No & NO & 0.6 & 0.1 & 0.1 & 20.1 & 0.8 & 0.1 \\
\hline ISOBUTYRALDEHYDE & ND & ND & 0.1 & $<0.1$ & ND & ND & 0.2 & $<0.1$ & 0.2 & $<0.1$ & 0.7 & 0.1 \\
\hline METHYL ETHYL KETONE & 1.20 & ND & 0.01 & 20.1 & No & No. & 0.2 & 20.1 & 0.2 & $<0.1$ & 0.7 & 01 \\
\hline BENZALDEHYDE & 0.9 & $<0.1$ & $<0.1$ & $<0.1$ & 0.5 & $<0.1$ & 0.2 & $<0.1$ & 0.5 & $<0.1$ & 0.1 & $<0.1$ \\
\hline HEXANALBE HYOE & $1=\mathrm{ND}$ & ND. & 1.2 .10 & ND. & $\mathrm{NO}$ & $\mathrm{NG}$ & 2.0 .1 & $<0.1$ & I. ND. &. $\mathrm{ND}$ & 1.08 & 0.1 \\
\hline SUMMATIONOFNON-METHANE COMPOUNDS & 10836.6 & 100.0 & 978.3 & 100.0 & 5198.0 & 100.0 & 933.6 & 99.9 & 5737.8 & 99.9 & 1155.2 & 99.8 \\
\hline
\end{tabular}




\section{APPENDIX M}

\section{AVERAGE SPECIATED EMISSIONS RESULTS FROM FTPS WITH METHANOL}


TABLE M-1. SUMMARY OF SPECIATED EXHAUST EMISSIONS FROM METHANOL

\begin{tabular}{|c|c|c|c|c|c|c|c|c|c|c|c|c|}
\hline \multirow[b]{4}{*}{ Compound } & \multicolumn{12}{|c|}{ Operating Conditlons } \\
\hline & \multicolumn{4}{|c|}{ Lean } & \multicolumn{4}{|c|}{ Stoichiometric } & \multicolumn{4}{|c|}{ Rich } \\
\hline & \multicolumn{2}{|c|}{$\begin{array}{l}\text { Without } \\
\text { Catalyst }\end{array}$} & \multicolumn{2}{|c|}{$\begin{array}{c}\text { With } \\
\text { Catalyst }\end{array}$} & \multicolumn{2}{|c|}{$\begin{array}{l}\text { Without } \\
\text { Catalyst }\end{array}$} & \multicolumn{2}{|c|}{$\begin{array}{c}\text { With } \\
\text { Catalyst }\end{array}$} & \multicolumn{2}{|c|}{$\begin{array}{l}\text { Without } \\
\text { Catalyst }\end{array}$} & \multicolumn{2}{|c|}{$\begin{array}{c}\text { With } \\
\text { Catalyst }\end{array}$} \\
\hline & $\begin{array}{c}\text { FTP } \\
\mathrm{mg} / \mathrm{mi}\end{array}$ & $\begin{array}{c}\text { NMOG } \\
\text { wt \% }\end{array}$ & $\begin{array}{c}\text { FTP } \\
\mathrm{mg} / \mathrm{mi}\end{array}$ & $\begin{array}{c}\text { NMOG } \\
\text { wt \% } \\
\end{array}$ & $\begin{array}{c}\text { FTP } \\
\mathrm{mg} / \mathrm{mi}\end{array}$ & $\begin{array}{c}\text { NMOG } \\
w+\% \\
\end{array}$ & $\begin{array}{c}\text { FTP } \\
\mathrm{mg} / \mathrm{mi}\end{array}$ & $\begin{array}{c}\text { NMOG } \\
\text { wt } \%\end{array}$ & $\begin{array}{c}\text { FTP } \\
\mathrm{mg} / \mathrm{mi}\end{array}$ & $\begin{array}{c}\text { NMOG } \\
\text { wt \% }\end{array}$ & $\begin{array}{c}\text { FTP } \\
\mathrm{mg} / \mathrm{mi}\end{array}$ & $\begin{array}{c}\text { NMOG } \\
\text { wt \% } \\
\end{array}$ \\
\hline METHANE & 14.1 & & 6.3 & & 27.5 & & 12.5 & & 27.5 & & 23.9 & \\
\hline ETHANE & 0.4 & 2.801 & 01 & $=0.1$ & 0.8 & 2.801 & 0.2 & $<0.1$ & 07 & $<0.1$ & 0.4 & $0.1 \%$ \\
\hline ETHYLENE & 7.4 & 0.1 & 0.3 & 0.1 & 10.6 & 0.2 & 0.6 & 0.1 & 7.5 & 0.2 & 0.3 & $<0.1$ \\
\hline PROPANE & $<0.1$ & 20.1 & 201 & 20,1 & $<0,1$ & 2.0 .1 & 2.20 .1 & 20.1 & 20.1 & 201 & .20 .1 & $\times 0.1$ \\
\hline PROPYLENE & 1.9 & $<0.1$ & 0.1 & $<0.1$ & 1.9 & $<0.1$ & 0.1 & $<0.1$ & 1.7 & $<0.1$ & 0.1 & $<0.1$ \\
\hline ACETYLENE & 27 & 80.1 & 0.8 & 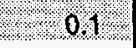 & 4.2 & 0.1 & 0.3 & 0.11 & 4.4. & 0.1 & 0.5 & 0.1 \\
\hline PROPADIENE & ND & ND & ND & ND & ND & ND & ND & ND & ND & ND & ND & ND \\
\hline BUTANE & 0.1 & $<0.1$ & 2.20 .1 & $=0.1$ & 0.1 & 20.1 & 20.1 & $<0.1$ & 0.1 & 20.1 & 0.1 & $<0.1$ \\
\hline TRANS-2-BUTENE & 0.2 & $<0.1$ & ND & ND & 0.1 & $<0.1$ & ND & ND & $<0.1$ & $<0.1$ & $<0.1$ & $<0.1$ \\
\hline भBUTENE & 0.3 & 20.1 & $=<0.1$ & $=0.1$ & 0.3 & $<011$ & .20 .1 & 2.01 & 02 & $<0.1$ & 20.1 & 2.201 \\
\hline 2-METHYLPROPENE (ISOBUTYLENE) & 0.9 & $<0.1$ & 0.1 & $<0.1$ & 0.7 & $<0.1$ & $<0.1$ & $<0.1$ & 0.7 & $<0.1$ & 0.1 & $<0.1$ \\
\hline 2;2.DIMETHYLPBOPANE (NEOPENTAN & $<0.1$ & 20,1 & ND. & ND & 20.1 & .2 .80 .1 & NB & ND & 20.1 & 20.1 & $<0.1$ & 20.1 \\
\hline PROPYNE & ND & ND & ND & ND & ND & ND & ND & ND & ND & ND & ND & ND \\
\hline 1,3 BUTADIENE & 0.6 & 20.1 & $2 \times 0.1$ & $=.201$ & 0.1 & $<0.1$. & .0 .1 & $<0,19$ & 0.4 & $<0.1$ & ND & ND. \\
\hline 2-METHYLPROPANE (ISOBUTANE) & ND & ND & ND & ND & $<0.1$ & $<0.1$ & ND & ND & 0.1 & $<0.1$ & $<0.1$ & $<0.1$ \\
\hline 1 BUTYNE & ND. & No & $1 \mathrm{ND}$ & ND & $\mathrm{ND}$ & ND & $.2 . \mathrm{NB}$ & $=.20$ & NO & NO & ND & ND \\
\hline METHANOL & 7860.0 & 91.0 & 623.1 & 95.4 & 4969.8 & 91.7 & 602.9 & 95.2 & 4403.5 & 92.1 & 747.3 & 95.2 \\
\hline CIS2:- & 0.1 & 20.1 & $1 . \mathrm{NO}$ & $\mathrm{ND}$ & 01 & 2.20 .1 & $<0.1$ & $<0.1$. & 20.1 & $<0.1$ & 2.80 .1 & 80.1 \\
\hline L-T-BUTENE & ND & ND & ND & ND & ND & ND & ND & ND & ND & ND & ND & ND \\
\hline ETHANOL & ND. & ND: & ND. & ND & ND & ND. & NB & NB & ND & NB & ND & ND. \\
\hline 2-METHYLBUTANE (ISOPENTANE) & 0.8 & $<0.1$ & $<0.1$ & $<0.1$ & 0.3 & $<0.1$ & $<0.1$ & $<0.1$ & 0.4 & $<0.1$ & 0.1 & $<0.1$ \\
\hline 2BUTYNE & NO & ND: & NO. & : & $\mathrm{NB}$ & No. & ND & NO & 1. NB & ND & ND & . NB. \\
\hline 1-PENTENE & ND & ND & ND & ND & 0.1 & $<0.1$ & ND & ND & 0.2 & $<0.1$ & 0.1 & $<0.1$ \\
\hline 2. METHYL-1 BUTENE & 0.2 & 20.1 & 0.1 & $20.1 \%$ & 80.1 & .0 .1 & 0.1 & 20.11 & 0.1 & 80.1 & 0.1 & 2011 \\
\hline PENTANE & 0.3 & $<0.1$ & 0.1 & $<0.1$ & 0.3 & $<0.1$ & 0.1 & $<0.1$ & 0.7 & $<0.1$ & 0.3 & $<0.1$ \\
\hline 2.METHYL I,3 BUTADIENE & 0.1. & 2.801 & .0 .1 & $<0.1$ & 0.1 & 20.1 & ND & No. & (20.1) & $<0.1$ & ND. & NO. \\
\hline TRANS-2-PENTENE & $<0.1$ & $<0.1$ & $<0.1$ & $<0.1$ & $<0.1$ & $<0.1$ & $<0.1$ & $<0.1$ & $<0.1$ & $<0.1$ & 0.1 & $<0.1$ \\
\hline 3,3-DIMETHYL-1.BUTENE & No & NB & ND & ND. & l. No & NB & No & ND & 0.1 & $<0: 1$ & ND & ND. \\
\hline CIS-2-PENTENE & 0.1 & $<0.1$ & 0.1 & $<0.1$ & 0.1 & $<0.1$ & $<0.1$ & $<0.1$ & $<0.1$ & $<0.1$ & $<0.1$ & $<0.1$ \\
\hline 2.METHYYL-2.RUIEENE & $=02$ & $=0.4=$ & 8. & $=0.1$ & 0.2 & $=0.1$ & 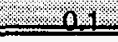 & 201. & 0.1 & $=0.4$ & 2.0 .11 & 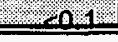 \\
\hline
\end{tabular}


TABLE M-1 (CONT'D). SUMMARY OF SPECIATED EXHAUST EMISSIONS FROM METHANOL.

\begin{tabular}{|c|c|c|c|c|c|c|c|c|c|c|c|c|}
\hline \multirow[b]{4}{*}{ Compound } & \multicolumn{12}{|c|}{ Operating Conditions } \\
\hline & \multicolumn{4}{|c|}{ Lean } & \multicolumn{4}{|c|}{ Stoichiometric } & \multicolumn{4}{|c|}{ Rich } \\
\hline & \multicolumn{2}{|c|}{$\begin{array}{l}\text { Without } \\
\text { Catalyst }\end{array}$} & \multicolumn{2}{|c|}{$\begin{array}{c}\text { With } \\
\text { Catalyst }\end{array}$} & \multicolumn{2}{|c|}{$\begin{array}{l}\text { Without } \\
\text { Catalyst }\end{array}$} & \multicolumn{2}{|c|}{$\begin{array}{c}\text { With } \\
\text { Catalyst }\end{array}$} & \multicolumn{2}{|c|}{$\begin{array}{l}\text { Without } \\
\text { Catalyst }\end{array}$} & \multicolumn{2}{|c|}{$\begin{array}{c}\text { With } \\
\text { Catalyst }\end{array}$} \\
\hline & $\begin{array}{c}\text { FTP } \\
\mathrm{mg} / \mathrm{ml}\end{array}$ & $\begin{array}{c}\text { NMOG } \\
\text { wt \% } \\
\end{array}$ & $\begin{array}{c}\text { FTP } \\
\mathrm{mg} / \mathrm{mi} \\
\end{array}$ & $\begin{array}{c}\text { NMOG } \\
w t \% \\
\end{array}$ & $\begin{array}{c}\text { FTP } \\
\mathrm{mg} / \mathrm{mi}\end{array}$ & $\begin{array}{c}\text { NMOG } \\
\text { wt \% } \\
\end{array}$ & $\begin{array}{c}\text { FTP } \\
\mathrm{mg} / \mathrm{mi}\end{array}$ & $\begin{array}{c}\text { NMOG } \\
\text { wt \% } \\
\end{array}$ & $\begin{array}{c}\text { FTP } \\
\mathrm{mg} / \mathrm{mi}\end{array}$ & $\begin{array}{c}\text { NMOG } \\
\text { wt \% } \\
\end{array}$ & $\begin{array}{c}\text { FTP } \\
\mathrm{mg} / \mathrm{mi}\end{array}$ & $\begin{array}{c}\text { NMOG } \\
\text { wt \% } \\
\end{array}$ \\
\hline CYCLOPENTADIENE & 0.2 & $<0.1$ & ND & ND & 0.1 & $<0.1$ & ND & ND & 0.1 & $<0.1$ & ND & ND \\
\hline 2.2. BIMETTYYLBUTANE & 02 & .40 .1 & 0.1 & $:<0.1$ & .0 .3 & 20.1 & 20.1 & 201 & 0.2 & $<0.1$ & 20.1 & .80 .1 \\
\hline CYCLOPENTENE & ND & ND & ND & ND & ND & ND & ND & ND & ND & ND & ND & ND \\
\hline 4.METHYL - I-PENTENE & 0.1 & 20.1. & No &. $\mathrm{ND}$ & ND & ND & . ND & ND. & $<0.1$ & 20.1 & $1 . \mathrm{NO}$ & No \\
\hline 3-METHYL-1-PENTENE & 0.1 & $<0.1$ & $<0.1$ & $<0.1$ & $<0.1$ & $<0.1$ & ND & ND & 0.1 & $<0.1$ & 0.1 & $<0.1$ \\
\hline OYGLOPENTANE & $\mathrm{NB}$ & .1 .10 & ND & ND & ND & NB & $\mathrm{NB}$ & $\mathrm{NB}$ & No & ND: & ND & ND \\
\hline 2,3-DIMETHYLBUTANE & 0.2 & $<0.1$ & $<0.1$ & $<0.1$ & 0.2 & $<0.1$ & $<0.1$ & $<0.1$ & 0.2 & $<0.1$ & 0.1 & $<0.1$ \\
\hline MTBE & ND & ND & ND & . No 1 & NO & ND. & . ND & ND & No & NB & ND & ND \\
\hline 2,3-DIMETHYL-1-BUTENE & ND & ND & ND & ND & ND & ND & ND & ND & ND & ND & ND & ND \\
\hline 4.METHYL-CIS 2 PENTENE & $1 \mathrm{NB}$ & ND & No & ND. & ND & NO & ND & No & ND & No & ND. & ND \\
\hline 2-METHYLPENTANE & 0.3 & $<0.1$ & 0.2 & $<0.1$ & 0.4 & $<0.1$ & 0.1 & $<0.1$ & 0.6 & $<0.1$ & 0.4 & $<0.1$ \\
\hline 4-METHYL-TRANS-2-PENTENE & ND & $\mathrm{ND}$ & ND & ND & NB & ND & ND & ND & NB & ND & ND & ND. \\
\hline 3-METHYLPENTANE & 0.3 & $<0.1$ & 0.4 & 0.1 & 0.3 & $<0.1$ & 0.1 & $<0.1$ & 0.3 & $<0.1$ & 0.5 & 0.1 \\
\hline 2.METHYL-1.PENTENE & 0.1 & $\times 0.1$ & ND & NO & 0.1 & $<01$ & $<0.1$ & 201 & $<0,1$ & 2.0 .1 & 20.1 & $<0.1$ \\
\hline 1-HEXENE & 0.1 & $<0.1$ & ND & ND & 0.1 & $<0.1$ & $<0.1$ & $<0.1$ & $<0.1$ & $<0.1$ & $<0.1$ & $<0.1$ \\
\hline HEXANE & 0.2 & $=0.1$ & 0.1 & 2.20 .1 & 0.2 & $=0.1$ & $=20.1$ & $=0.1$ & 0.2 & $<0.1$ & 02 & $<01$ \\
\hline TRANS-3-HEXENE & ND & ND & ND & ND & ND & ND & ND & ND & 0.1 & $<0.1$ & 0.1 & $<0.1$ \\
\hline CIS-3HEXENE & 0.2 & 201 & 0.1 & $<0.1$ & 0.2 & 20.1 & 20.1 & 80.1 & 20.1 & $2 ., 20,1$ & 20.1 & $2<0.1$ \\
\hline TRANS-2-HEXENE & ND & ND & ND & NO & ND & ND & ND & ND & ND. & ND & ND & ND \\
\hline 3.METHYL-TRANS-2-PENTENE & 1.01 & 20.1 ? & NO & ND & 01 & $=<0.1$ & ND & NB. & 0.1 & .00 .1 & 0.1 & 20.1 \\
\hline 2-METHYL-2-PENTENE & ND & ND & ND & ND & ND & ND & ND & ND & 0.1 & $<0.1$ & $<0.1$ & $<0.1$ \\
\hline 8.METHYLCYCLOPENTENE & $\mathrm{NB}$ & ND. & ND & ND & No & NO & NB & NO & $\mathrm{NB}$ & ND & NB & NB \\
\hline CIS-2-HEXENE & $<0.1$ & $<0.1$ & ND & ND & ND & ND & ND & ND & 0.1 & $<0.1$ & 0.1 & $<0.1$ \\
\hline ETBE & NO & $.2 \mathrm{NB}$ & ND & ND & NO & NO & ND & ND. & ND & $\mathrm{ND}$ ? & NO & ND \\
\hline 3-METHYL-CIS-2-PENTENE & $<0.1$ & $<0.1$ & 0.1 & $<0.1$ & 0.1 & $<0.1$ & $<0.1$ & $<0.1$ & 0.1 & $<0.1$ & 0.1 & $<0.1$ \\
\hline 2,2.DIMETHYLPENTANE & $0.1 \%$ & 20.1 & 20.1 & $<0.1$ & 0.1 & $<0.1$ & 20.1 & 20.1 & 02 & $<0.1$ & $<01$ & 201 \\
\hline METHYLCYCLOPENTANE & ND & ND & ND & ND & ND & ND & ND & ND & ND & ND & ND & ND \\
\hline 2,4 DIMETHYLPENTANE & 0.3 & 20.1 & 0.1 & $<0,1$ & 0.2 & 50.1 & $<0.1$ & 201 & 0.1 & 20.1 & 0.1 & 20.1 \\
\hline 2,3,3-TRIMETHYL-1-BUTENE & ND & ND & ND & ND & ND & ND & ND & ND & ND & ND & ND & ND \\
\hline 2.2.3.TRIMETHYYBOUTANE & 0.2. & $\times 0.1 .1$ & 20.1 & 20.1 .1 & $=0.1$ & 20.1 .1 & 2.0 .1 & 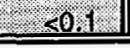 & 20.1 & 20.1 & $2 .<01:$ & $\leq 0.1$ \\
\hline
\end{tabular}




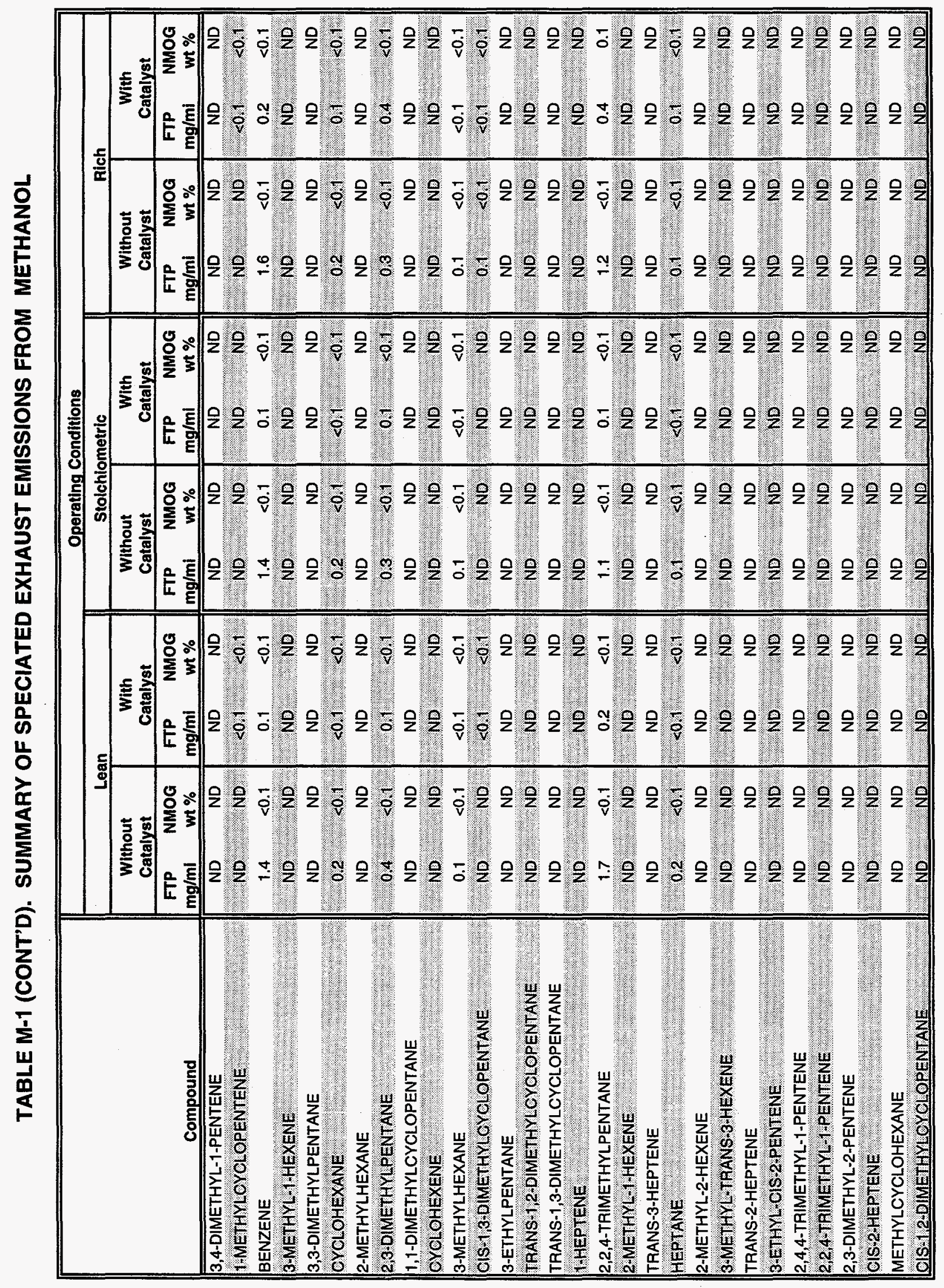


TABLE M-1 (CONT'D). SUMMARY OF SPECIATED EXHAUST EMISSIONS FROM METHANOL

\begin{tabular}{|c|c|c|c|c|c|c|c|c|c|c|c|c|}
\hline \multirow[b]{4}{*}{ Compound } & \multicolumn{12}{|c|}{ Operating Conditions } \\
\hline & \multicolumn{4}{|c|}{ Lean } & \multicolumn{4}{|c|}{ Stoichlometric } & \multicolumn{4}{|c|}{ Rich } \\
\hline & \multicolumn{2}{|c|}{$\begin{array}{l}\text { Without } \\
\text { Catalyst }\end{array}$} & \multicolumn{2}{|c|}{$\begin{array}{c}\text { With } \\
\text { Catalyst }\end{array}$} & \multicolumn{2}{|c|}{$\begin{array}{l}\text { Without } \\
\text { Catalyst }\end{array}$} & \multicolumn{2}{|c|}{$\begin{array}{c}\text { With } \\
\text { Catalyst }\end{array}$} & \multicolumn{2}{|c|}{$\begin{array}{l}\text { Without } \\
\text { Catalyst }\end{array}$} & \multicolumn{2}{|c|}{$\begin{array}{c}\text { With } \\
\text { Catalyst }\end{array}$} \\
\hline & $\begin{array}{c}\text { FTP } \\
\mathrm{mg} / \mathrm{mi}\end{array}$ & $\begin{array}{c}\text { NMOG } \\
\text { wt \% }\end{array}$ & $\begin{array}{c}\text { FTP } \\
\mathrm{mg} / \mathrm{mi}\end{array}$ & $\begin{array}{c}\text { NMOG } \\
\text { wt \% }\end{array}$ & \begin{tabular}{|c} 
FTP \\
$\mathrm{mg} / \mathrm{mi}$
\end{tabular} & $\begin{array}{c}\text { NMOG } \\
\text { wt \% }\end{array}$ & $\begin{array}{c}\text { FTP } \\
\mathrm{mg} / \mathrm{mi}\end{array}$ & $\begin{array}{c}\text { NMOG } \\
\text { wt } \% \\
\end{array}$ & $\begin{array}{c}\text { FTP } \\
\mathrm{mg} / \mathrm{mi}\end{array}$ & $\begin{array}{c}\text { NMOG } \\
\text { wt \% }\end{array}$ & $\begin{array}{c}\text { FTP } \\
\mathrm{mg} / \mathrm{mi}\end{array}$ & $\begin{array}{c}\text { NMOG } \\
\text { wt \% }\end{array}$ \\
\hline 2,2-DIMETHYLHEXANE & 0.3 & $<0.1$ & $<0.1$ & $<0.1$ & 0.1 & $<0.1$ & $<0.1$ & $<0.1$ & 0.2 & $<0.1$ & 0.1 & $<0.1$ \\
\hline 1,1,3-TRIMETHMLOYCLOPENTANE & NB & NB & ND & ND & No & NB & No & NB & NB & No & ND. & NO \\
\hline 2,4,4-TRIMETHYL-2-PENTENE & ND & ND & ND & ND & ND & ND & ND & ND & ND & ND & ND & ND \\
\hline 2,2,3-TRIMETHYUPENTANE & $\mathrm{ND}$ & ND. & ND. & : $: 2$ & ND. & ND & ND & ND & ND. & NB: & ND. & ND \\
\hline 2,5-DIMETHYLHEXANE & 0.0 & ND & ND & ND & ND & ND & ND & ND & ND & ND & ND & ND \\
\hline ETHYLCYCLOPENTANE & ND. & ND & ND & ND & ND & ND: & No & NB & NB & ND & ND & NB \\
\hline 2,4-DIMETHYLHEXANE & 0.3 & $<0.1$ & 0.1 & $<0.1$ & 0.2 & $<0.1$ & ND. & ND & 0.2 & $<0.1$ & 0.1 & $<0.1$ \\
\hline 1-TRANS-2-GS-4-TRIMETHYLOYCLOPENTANE & ND & $\mathrm{NO}$ & ND & $\mathrm{ND}$ & No & ND & No & ND & NB. & No & NO & NO \\
\hline 3,3-DIMETHYLHEXANE & ND & ND & ND & ND & ND & ND & ND & ND & ND & ND & ND & ND \\
\hline 1-TRANS-2-GIS-3 TRIMETHYLCYCLOPENTANE & ND & ND. & No & NO & No & No & ND & ND & ND & No & ND. & ND \\
\hline 2,3,4-TRIMETHYLPENTANE & ND & ND & ND & ND & ND & ND & ND & ND & ND & ND & ND & ND \\
\hline 2,3,3.TAIMETHYLPENTANE & 0.5 & 20.1 & -0.1 & 50.1 & 0.4 & 80.1 . & 20.1 & 80.1 & 03 & 20.1 & 0.1 & 20.11 \\
\hline TOLUENE & 2.9 & $<0.1$ & 0.7 & 0.1 & 0.6 & $<0.1$ & 0.2 & $<0.1$ & 1.8 & $<0.1$ & 0.7 & 0.1 \\
\hline 2,3.DIMETHYLHEXANE & 0.4 & .20 .1 & NO & No & ND. & ND: & ND & ND & 0.1 & 20.1 & NO & no \\
\hline 1,1,2-TRIMETHYLCYCLOPENTANE & ND & ND & ND & ND & ND & ND & ND & ND & ND & ND & ND & ND \\
\hline 2.METHYLHEPTANE & ND & ND & $=0.1$ & 20.1 & ND. & ND. & NB & ND & NB. & ND & ND & ND \\
\hline 3,4-DIMETHYLHEXANE & ND & ND & ND & ND & ND & ND & ND & ND & ND & ND & ND & ND \\
\hline 2,2,4,4 TETRAMETHYLPENTANE & No & $\mathrm{NB}$ & $\mathrm{NO}$ & No & No. & NB & NO & ND & ND & NO & NO & ND \\
\hline 4-METHYLHEPTANE & ND & ND & ND & ND & ND & ND & ND & ND & ND & ND & ND & ND \\
\hline 2METHYL-3 ETHYLPENTANE & ND & ND & ND & ND & ND & ND & ND & ND. & ND & ND & ND & ND \\
\hline 2,6-DIMETHYLHEPTANE & ND & ND. & ND & ND & ND & ND & ND & ND & ND & ND & ND & ND \\
\hline 3 METHYLHEPTANE & ND & ND & $<0.1$ & 20.1 & ND. & ND & NB. & No & <0.1. & 201. & ND & NO \\
\hline 1-CIS,2-TRANS,3-TRIMETHYLCYCLOPENTANE & ND & ND & ND & ND & ND & ND & ND & ND & ND & ND & ND & ND \\
\hline CIS.1.3-DIMETHYLCYCLOHEXANE & NO & NO & ND. & ND & ND & ND. & No & ND. & NB & ND & ND & ND \\
\hline TRANS-1,4-DIMETHYLCYCLOHEXANE & 0.1 & $<0.1$ & ND & ND & 0.1 & $<0.1$ & ND & ND & $<0.1$ & $<0.1$ & ND & ND \\
\hline 3ETHMLHEXANE & ND & ND: & ND & ND & ND. & NB & NB & NO & No & No & NB & ND \\
\hline 2,2,5-TRIMETHYLHEXANE & ND & ND & ND & ND & ND & ND & ND & ND & ND & ND & ND & ND \\
\hline CIS-1 METHYL-3-ETHYLGYCLOPENTANE & ND & NB & ND & ND & NO & ND & ND & $\mathrm{ND}$ & $1 . \times \mathrm{NB}$ & $\mathrm{ND}$ & No & NB \\
\hline 1,1-DIMETHYLCYCLOHEXANE & ND & ND & ND & ND & ND & ND & ND & ND & ND & ND & ND & ND \\
\hline TRANS, 1 METHYL_2 ETHHYLOYCLOPENT & ND & ND & ND & ND. & ND. & NB & 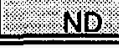 & NO & ND & $N D$ & 1 ND & ND \\
\hline
\end{tabular}


TABLE M-1 (CONT'D). SUMMARY OF SPECIATED EXHAUST EMISSIONS FROM METHANOL

\begin{tabular}{|c|c|c|c|c|c|c|c|c|c|c|c|c|}
\hline \multirow[b]{4}{*}{ Compound } & \multicolumn{12}{|c|}{ Operating Conditions } \\
\hline & \multicolumn{4}{|c|}{ Lean } & \multicolumn{4}{|c|}{ Stoichlometric } & \multicolumn{4}{|c|}{ Rich } \\
\hline & \multicolumn{2}{|c|}{$\begin{array}{l}\text { Without } \\
\text { Catalyst }\end{array}$} & \multicolumn{2}{|c|}{$\begin{array}{c}\text { With } \\
\text { Catalyst }\end{array}$} & \multicolumn{2}{|c|}{$\begin{array}{l}\text { Without } \\
\text { Catalyst }\end{array}$} & \multicolumn{2}{|c|}{$\begin{array}{c}\text { With } \\
\text { Catalyst }\end{array}$} & \multicolumn{2}{|c|}{$\begin{array}{l}\text { Without } \\
\text { Catalyst }\end{array}$} & \multicolumn{2}{|c|}{$\begin{array}{c}\text { With } \\
\text { Catalyst }\end{array}$} \\
\hline & $\begin{array}{l}\text { FTP } \\
\mathrm{mg} / \mathrm{mi}\end{array}$ & $\begin{array}{c}\text { NMOG } \\
w t \%\end{array}$ & $\begin{array}{c}\text { FTP } \\
\mathrm{mg} / \mathrm{mi}\end{array}$ & $\begin{array}{c}\text { NMOG } \\
\text { wt \% }\end{array}$ & $\begin{array}{c}\text { FTP } \\
\mathrm{mg} / \mathrm{mi}\end{array}$ & $\begin{array}{c}\text { NMOG } \\
\text { wt \% }\end{array}$ & $\begin{array}{c}\text { FTP } \\
\mathrm{mg} / \mathrm{mi}\end{array}$ & $\begin{array}{c}\text { NMOG } \\
\text { wt \% }\end{array}$ & $\begin{array}{c}\text { FTP } \\
\mathrm{mg} / \mathrm{mi}\end{array}$ & $\begin{array}{c}\text { NMOG } \\
\text { wt \% }\end{array}$ & $\begin{array}{c}\mathrm{FTP} \\
\mathrm{mg} / \mathrm{mi}\end{array}$ & $\begin{array}{c}\text { NMOG } \\
\text { wt \% }\end{array}$ \\
\hline 1-METHYL-1-ETHYL-CYCLOPENT & ND & ND & ND & ND & ND & ND & ND & ND & ND & ND & ND & ND \\
\hline 2,4,4-TAIMETHYLHEXANE & ND & 1.20 & ND & NB & ND & ND & ND & No & ND. & NB & $1 . \mathrm{NB}$ & NB. \\
\hline 2,2,4-TRIMETHYLHEXANE & ND & ND & ND & ND & ND & ND & ND & ND & ND & ND & ND & ND \\
\hline TRANS 1, 2DDMETHYLOYCLOHEXAN & NB & ND & ND & ND & ND & No. & ND & ND. & ND & $=. \mathrm{ND}$ & 1. NO & ND. \\
\hline 1-OCTENE & ND & ND & ND & ND & ND & ND & ND & ND & ND & ND & ND & ND \\
\hline TRANS-4-OGTENE & ND & ND & ND & NO: & ND & ND: & . ND & No & ND & $\%$ NB & ND & . NB. \\
\hline OCTANE & ND & ND & ND & ND & ND & ND & ND & ND & $<0.1$ & $<0.1$ & ND & ND \\
\hline TRANS 2 -OCTENE & ND. & $\mathrm{NB}$ & $\% . \mathrm{NB}$ & . ND. & $\mathrm{NO}$ & NO & ND. & ND & NB & $\mathrm{ND}$ & No & NO \\
\hline TRANS-1,3-DIMETHYLCYCLOHEX & ND & ND & ND & ND & ND & ND & ND & ND & ND & ND & ND & ND \\
\hline CIS-1,4 DIMETHYLOYCLOHEXAN & ND & ND. & No & ND & NB & NB. & ND & NO: & ND. & ND & ND & ND \\
\hline CIS-2-OCTENE & ND & ND & ND & ND & ND & ND & ND & ND & ND & ND & ND & ND \\
\hline 2,3,5.TRMETHYLHEXANE & ND: & ND & 1.20 & NO & $\mathrm{ND}$ & NO & No & ND. & No & $\mathrm{ND}$ & No & No \\
\hline L-2-ETI & ND & ND & ND & ND & ND & ND & ND & ND & ND & ND & ND & ND \\
\hline 2.METHYL-2-ETHYLHEPTANE & ND & ND & No & . ND. & ND & ND. & ND & ND & ND & ND & ND. & $\mathrm{ND}$ \\
\hline 2,4-DIMETHYLHEPTANE & 0.4 & $<0.1$ & 1.0 & 0.2 & 0.6 & $<0.1$ & 1.0 & 0.2 & 0.5 & $<0.1$ & 0.9 & 0.1 \\
\hline 4,4-DIMETHYLHEPTANE & NB & ND & No & ND & $\mathrm{ND}$ & $\mathrm{NB}$ & NB & ND & 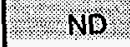 & No. & ND. & ND \\
\hline CIS-1,2-DIMETHYLCYCLOHEXANE & ND & ND & ND & ND & ND & ND & ND & ND & ND & ND & ND & ND \\
\hline ETHYLCYCLOHEXANE & ND & $\mathrm{ND}$ & $\mathrm{ND}$ & NB & $\mathrm{NB}$ &. $\mathrm{NO}$ & NB & ND & $\mathrm{NB}$ & $\mathrm{ND}$ & No & NO \\
\hline PROPYLCYCLOHEXANE & ND & ND & ND & ND & ND & ND & ND & ND & ND & ND & ND & ND \\
\hline 2.METHYL-4-ETHYLHEXANE: & ND & ND & . No & NB & No & No & ND & ND & No & $\mathrm{ND}$ & NO & NB \\
\hline 2,6-DIMETHYLHEPTANE & ND & ND & ND & ND & ND & ND & ND & ND & ND & ND & ND & ND \\
\hline 1, 1,3 TRIMETHYL OYCLOHEXANE & ND. & ND. & ND & NB & $\mathrm{ND}$ & NB & NB. & No & $\mathrm{NB}$ & ND & $\mathrm{NB}$ & ND \\
\hline 2,5-DIMETHYLHEPTANE & ND & ND & ND & ND & ND & ND & ND & ND & ND & ND & ND & ND \\
\hline 3.3 DIMETHVIHEPTANE & NB & ND & ND & No & ND & ND & ND & $\mathrm{ND}$ & ND & ND & NO & No \\
\hline 3,5-DIMETHYLHEPTANE & ND & ND & ND & ND & ND & ND & ND & ND & ND & ND & ND & ND \\
\hline ETHYLBENZENE & 0.2 & $<01$ & 80.1 & $<01$ & 0.2 & 20.1 & 80.1 & 80.1 & 0.2 & 280.1 & 201 & 80.1 \\
\hline 2,3-DIMETHYLHEPTANE & ND & ND & ND & ND & ND & ND & ND & ND & ND & ND & ND & ND \\
\hline m. \& p XYLENE & 0.6 & $<01$ & 1.0 .1 & $<0.1$ & .0 .3 & .80 .1 & 0.1 & $\times 0.1$ : & $=0.4$ & $=20.1$ & 0.1 & 20.1 \\
\hline 4-METHYLOCTANE & ND & ND & ND & ND & ND & ND & ND & ND & ND & ND & ND & ND \\
\hline 2.METHYLOCTANE & $1.0 \mathrm{NB}$ & ND & D. ND & $2 . \mathrm{ND}$ & ND & NB & $3 . \mathrm{ND}$ & $\mathrm{ND}$ & 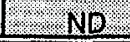 & $\mathrm{ND}$ & ND & $\mathrm{ND}$ \\
\hline
\end{tabular}


TABLE M-1 (CONT'D). SUMMARY OF SPECIATED EXHAUST EMISSIONS FROM METHANOL

\begin{tabular}{|c|c|c|c|c|c|c|c|c|c|c|c|c|}
\hline \multirow[b]{4}{*}{ Compound } & \multicolumn{12}{|c|}{ Operating Conditions } \\
\hline & \multicolumn{4}{|c|}{ Lean } & \multicolumn{4}{|c|}{ Stoichiometric } & \multicolumn{4}{|c|}{ Rich } \\
\hline & \multicolumn{2}{|c|}{$\begin{array}{l}\text { Without } \\
\text { Catalyst }\end{array}$} & \multicolumn{2}{|c|}{$\begin{array}{c}\text { With } \\
\text { Catalyst }\end{array}$} & \multicolumn{2}{|c|}{$\begin{array}{l}\text { Without } \\
\text { Catalyst }\end{array}$} & \multicolumn{2}{|c|}{$\begin{array}{c}\text { With } \\
\text { Catalyst }\end{array}$} & \multicolumn{2}{|c|}{$\begin{array}{l}\text { Without } \\
\text { Catalyst }\end{array}$} & \multicolumn{2}{|c|}{$\begin{array}{c}\text { With } \\
\text { Catalyst }\end{array}$} \\
\hline & $\begin{array}{c}\text { FTP } \\
\mathrm{mg} / \mathrm{mi}\end{array}$ & $\begin{array}{l}\text { NMOG } \\
\text { wt \% }\end{array}$ & $\begin{array}{c}\text { FTP } \\
\mathrm{mg} / \mathrm{ml}\end{array}$ & $\begin{array}{c}\text { NMOG } \\
\text { wt \% } \\
\end{array}$ & $\begin{array}{c}\text { FTP } \\
\mathrm{mg} / \mathrm{mi}\end{array}$ & $\begin{array}{c}\text { NMOG } \\
\text { wt \% } \\
\end{array}$ & $\begin{array}{c}\text { FTP } \\
\mathrm{mg} / \mathrm{mi}\end{array}$ & $\begin{array}{c}\text { NMOG } \\
\text { wt \% }\end{array}$ & $\begin{array}{c}\text { FTP } \\
\mathrm{mg} / \mathrm{mi}\end{array}$ & $\begin{array}{c}\text { NMOG } \\
w t \% \\
\end{array}$ & $\begin{array}{c}\text { FTP } \\
\mathrm{mg} / \mathrm{ml} \\
\end{array}$ & $\begin{array}{c}\text { NMOG } \\
\text { wt } \% \\
\end{array}$ \\
\hline 3-METHYLOCTANE & ND & ND & ND & $\mathrm{ND}$ & ND & ND & ND & ND & ND & ND & ND & ND \\
\hline STYRENE & $2 . \mathrm{NO}$ & NB & No & ND. & ND & ND. & ND & NB. & No & No & ND & $\mathrm{ND}$ \\
\hline O-XYLENE & 0.2 & $<0.1$ & $<0.1$ & $<0.1$ & 0.1 & $<0.1$ & 0.1 & $<0.1$ & 0.3 & $<0.1$ & 0.1 & $<0.1$ \\
\hline 2,4,6-TRIMETHYUHEXANE & ND. & NB & No & NE. & ND & NB & ND. & ND & ND & No & ND & No \\
\hline 1-NONENE & ND & ND & ND & ND & ND & ND & ND & ND & ND & ND & ND & ND \\
\hline NONANE: & ND. & $=\mathrm{NO}$ & ND & No & ND & ND & ND & ND & NO & ND & ND & NB \\
\hline ISOPROPYLBENZENE (CUMENE) & ND & ND & ND & ND & ND & ND & ND & ND & $<0.1$ & $<0.1$ & ND & ND \\
\hline 2,2.DIMETHYLOCTANE & 1. ND & NB. & ND & NO & ND & NB & ND & NO & ND & No & ND. & ND \\
\hline 2,4-DIMETHYLOCTANE & ND & ND & ND & ND & ND & ND & ND & ND & $<0.1$ & $<0.1$ & ND & ND \\
\hline ПPROPYLBENZENE & NO. & NO & 40.1 & 1.20 .1 & NO & $\mathrm{NO}$ & NO & ND & 20.1 & 20.1 & $\mathrm{NO}$ & NB \\
\hline 1-METHYL-3-ETHYLBENZENE & 0.3 & $<0.1$ & ND & ND & 0.2 & $<0.1$ & $<0.1$ & $<0.1$ & 0.3 & $<0.1$ & 0.1 & $<0.1$ \\
\hline I METHYL-4 ETHYLBENZENE & 0.1 & 20.1 & ND & No & 0.2 & $<01$ & 20 & 80.1 & 0.1 & $<0.1$ & ND & No \\
\hline 1,3,5-TRIMETHYLBENZENE & 0.1 & $<0.1$ & ND & ND & 0.1 & $<0.1$ & ND & ND & 0.1 & $<0.1$ & $<0.1$ & $<0.1$ \\
\hline 1 - METHYL 2 ETHYLBENZENE & $<0.1$ & $<0.1$ & $1 . N B$ & NB & 20.1 & 2.20 .1 & 20.1 & $\times 0.1$ & 80.1 & $<0.1$ & ND & $\mathrm{NB}$ \\
\hline 1,2,4-TRIMETHYLBENZENE & 0.6 & $<0.1$ & 0.2 & $<0.1$ & 0.3 & $<0.1$ & 0.1 & $<0.1$ & 0.6 & $<0.1$ & 0.1 & $<0.1$ \\
\hline DECANE & 0.2 & 20.1 & 01 & 20.1 & 0.1 & $<0.1$ & 0.1 & 20.1 & 0.1 & $<0.1$ & .02 & $<0.1$ \\
\hline ISOBUTYLBENZENE & 0.2 & $<0.1$ & 0.1 & $<0.1$ & $<0.1$ & $<0.1$ & 0.1 & $<0.1$ & 0.1 & $<0.1$ & 0.1 & $<0.1$ \\
\hline METHYLPROPYLBENZENE & $=\mathrm{NB}$ & NB & No & ND & ND & ND & ND & ND & No & NO & No & NO \\
\hline S-BUTYLBENZENE & ND & ND & ND & ND & ND & ND & ND & ND & ND & ND & ND & ND \\
\hline 1-METHYL 3 ISOPROPYL BENZENE. & 20.1 & $<0.1$ & ND & ND. & ND & $\mathrm{ND}$ & . NO & No & 20.1 & $<0.1$ & ND & NB \\
\hline 1,2,3-TRIMETHYLBENZENE & ND & ND & ND & ND & ND & ND & ND & ND & ND & ND & ND & ND \\
\hline 1.METHYL-4.ISOPROPYLBENZENE & ND & ND & NO & NB & No. & $\mathrm{NB}$ & No & ND & ND & No & ND & ND \\
\hline IND AN & ND & ND & ND & ND & ND & ND & ND & ND & ND & ND & ND & ND \\
\hline 1.METHYL-2 ISOPHOPYLBENZENE & ND & ND & NB. & NO & ND & ND & NB & ND & $\mathrm{ND}$ & No ? & No. & NO: \\
\hline 1,3-DIETHYLBENZENE & ND & ND, & ND & ND & ND & ND & ND & ND & ND & ND & ND & ND \\
\hline 1.4 DIETHYLBENZENE & ND. & ND & ND & ND. & ND & ND & NB & No & NB & ND & ND & NB \\
\hline 1-METHYL-3-N-PROPYLBENZENE & ND & NO & ND & ND & ND & ND & ND & ND & ND & ND & ND & ND \\
\hline 1.METHYL-4 N-PROPYLBENZENE & NB. & $\mathrm{NB}$ & $\mathrm{ND}$ & No & NB & $\mathrm{NO}$ & $\mathrm{NB}$ & ND & ND & ND & NO & ND \\
\hline 1,2 DIETHYLBENZENE & ND & ND & ND & ND & ND & ND & ND & ND & ND & ND & ND & ND \\
\hline 1. METHYL-2N-PBOPYLBENZENE & NO & NB: & ND & $=\mathrm{ND}$ & $2 . \mathrm{NB}$ & ND & $=\mathrm{NB}$ & ND. & $2 . \mathrm{NB}$ & $\mathrm{NB}$ & $2 . \mathrm{NB}$ & ND \\
\hline
\end{tabular}


TABLE M-1 (CONT'D). SUMMARY OF SPECIATED EXHAUST EMISSIONS FROM METHANOL

\begin{tabular}{|c|c|c|c|c|c|c|c|c|c|c|c|c|}
\hline \multirow[b]{4}{*}{ Compound } & \multicolumn{12}{|c|}{ Operating Conditions } \\
\hline & \multicolumn{4}{|c|}{ Lean } & \multicolumn{4}{|c|}{ Stolchiometric } & \multicolumn{4}{|c|}{ Rich } \\
\hline & \multicolumn{2}{|c|}{$\begin{array}{l}\text { Without } \\
\text { Catalyst }\end{array}$} & \multicolumn{2}{|c|}{$\begin{array}{c}\text { With } \\
\text { Catalyst }\end{array}$} & \multicolumn{2}{|c|}{$\begin{array}{l}\text { Without } \\
\text { Catalyst }\end{array}$} & \multicolumn{2}{|c|}{$\begin{array}{c}\text { With } \\
\text { Catalyst }\end{array}$} & \multicolumn{2}{|c|}{$\begin{array}{l}\text { Without } \\
\text { Catalyst }\end{array}$} & \multicolumn{2}{|c|}{$\begin{array}{c}\text { With } \\
\text { Catalyst }\end{array}$} \\
\hline & \begin{tabular}{|c} 
FTP \\
$\mathrm{mg} / \mathrm{mi}$
\end{tabular} & $\begin{array}{c}\text { NMOG } \\
w t \% \\
\end{array}$ & $\begin{array}{c}\text { FTP } \\
\mathrm{mg} / \mathrm{mi}\end{array}$ & $\begin{array}{c}\text { NMOG } \\
w t \% \\
\end{array}$ & $\begin{array}{c}\text { FTP } \\
\mathrm{mg} / \mathrm{mi}\end{array}$ & $\begin{array}{c}\text { NMOG } \\
\text { wt \% } \\
\end{array}$ & $\begin{array}{c}\mathrm{FTP} \\
\mathrm{mg} / \mathrm{ml}\end{array}$ & $\begin{array}{c}\text { NMOG } \\
\text { wt \% } \\
\end{array}$ & $\begin{array}{r}\mathrm{FTP} \\
\mathrm{mg} / \mathrm{mi}\end{array}$ & $\begin{array}{c}\text { NMOG } \\
\text { wt \% }\end{array}$ & $\begin{array}{c}\begin{array}{c}\text { FTP } \\
\mathrm{mg} / \mathrm{ml}\end{array} \\
\end{array}$ & $\begin{array}{c}\text { NMOG } \\
\text { wt \% } \\
\end{array}$ \\
\hline 1,4-DIMETHYL-2-ETHYLBENZENE & ND & ND & ND & ND & ND & ND & ND & ND & $\mathrm{ND}$ & ND & ND & ND \\
\hline 1,3-DIMETHYL 4 ETHYLBENZENE & NB & ND & ND & $\mathrm{ND}$ & ND & ND. & ND. & $\mathrm{NO}$ & ND. & NO & $1 . \mathrm{NB}$ & No. \\
\hline 1,2-DIMETHYL-4-ETHYLBENZENE & ND & ND & ND & ND & ND & ND & ND. & ND & ND & ND & ND & ND \\
\hline 1.3.DIMETHYL-2.ETHYLBENZENE & ND & NB & ND & No & NO & ND & $1.2 \mathrm{ND}$ & ND & ND & $\mathrm{NB}$ & ND & ND \\
\hline UND ECANE & $<0.1$ & $<0.1$ & ND & ND & $<0.1$ & $<0.1$ & 0.1 & $<0.1$ & ND & ND & ND & ND \\
\hline 1.2-DIMETHYL-3-ETHYLBENZENE & $\mathrm{ND}$ &. NO & ND & ND & ND & $\mathrm{NB}$ & No & No & ND & No & ND & $\mathrm{NB}$ \\
\hline 1,2,4,5-TETRAMETHYLBENZENE & ND & ND & ND & ND & ND & ND & ND & ND & ND & ND & ND & ND \\
\hline 2. METHYLBUTYLBENZENE ISEC AMYL & ND & ND & ND & NO & ND & ND & ND. & ND & ND & ND. & ND & No \\
\hline 3,4 DIMETHYLCUMENE & ND & ND & ND & ND & ND & ND & ND & ND & ND & ND & ND & ND \\
\hline $1,2,3,5$ TETRAMETHYLBENZENE & $1 . \mathrm{NB}$ & ND & NO & ND & $\mathrm{No}$ & $=\mathrm{NB}$ & ND. & $.2 . \mathrm{NB}$ & ND & ND & $\mathrm{ND}$ & $\mathrm{NO}$ \\
\hline TERT-1-BUT-2-METHYLBENZENE & ND & ND & ND & ND & ND & ND & ND & ND & ND & ND & ND & ND \\
\hline$[1,2,3,4$ TETRAMETHYLBENZENE & 1. ND. & NO & NO & NO & No & $.2 \mathrm{NB}$. & NO & ND & No & No & No. & NB. \\
\hline N.PENT-BENZENE & ND & ND & ND & ND & ND & ND & ND & ND & ND & ND & ND & ND \\
\hline TERT-1-BUT-3;5-DIMETHYLBENZEI & ND & ND & ND & $=\mathrm{NB}$ & $\mathrm{NB}$ & ND: & ND: & ND & ND & NB & $\mathrm{NO}$ & ND. \\
\hline NAPHTHALENE & ND & ND & ND & ND & ND & ND & ND & ND & ND & ND & ND & ND \\
\hline DODECANE & ND & ND & ND & ND & $\mathrm{NB}$ & $\mathrm{ND}$ & ND & ND & NB & ND. & ND & ND \\
\hline FORMALDEHYDE & 736.1 & 8.5 & 23.9 & 3.7 & 409.3 & 7.6 & 24.4 & 3.8 & 343.8 & 7.2 & 29.7 & 3.8 \\
\hline ACETALDEHYDE & 6.3 & .0 .1 & 0.1 & (.) 80.1 & 8.7 & 0.2 & 0.9 & \%.1. & 19 & 1.801 & 0.1 & 20.1 \\
\hline ACROLEIN & 0.8 & $<0.1$ & ND & ND & 1.4 & $<0.1$ & $<0.1$ & $<0.1$ & 0.3 & $<0.1$ & ND & ND \\
\hline ACETONE & 0.1 & $=00.1$ & 8.0 .1 & 20.1 & 0.1 & $=80.1$ & 20.1 & 20.1 & $<0.1$ & $<0.1$ & $<0.1$ & 20.1 \\
\hline |PROPIONALDEHYDE & 0.1 & $<0.1$ & $<0.1$ & $<0.1$ & 0.2 & $<0.1$ & $<0.1$ & $<0.1$ & $<0.1$ & $<0.1$ & $<0.1$ & $<0.1$ \\
\hline CROTONALDEHYDE & ND & .10 & No & $=\mathrm{NO}$ ! & ND & No & No & NB. & NO & ND & ND & NO \\
\hline ISOBUTYRALDEHYDE & ND & ND & $<0.1$ & $<0.1$ & ND & ND & $<0.1$ & $<0.1$ & ND & ND & $<0.1$ & $<0.1$ \\
\hline METHYL ETHYL KETONE & ND. & ND & $1 .<0.1$ & $<0.1$ & No & $\mathrm{NB}$ & $<0.1$ & 20.1 & ND. & ND & 20,1 & 20.1 \\
\hline BEENZALDEHYDE & 0.5 & $<0.1$ & ND & ND & 0.1 & $<0.1$ & 0.5 & 0.1 & $<0.1$ & $<0.1$ & $<0.1$ & $<0.1$ \\
\hline HEXANALDEHYYDE & $\mathrm{ND}$ & $\mathrm{NO}^{2}$ & No & ND & ND & $.2 \mathrm{ND}$ & D.: ND. & ND & ND & ND. & $\mathrm{ND}$ & $\mathrm{ND}$ \\
\hline SUMMATION OF NON-METHANE COMPOUNDS & 8634.5 & 99.9 & 653.0 & 99.9 & 5418.6 & 100.0 & 633.1 & 100.0 & 4778.9 & 100.0 & 785.1 & 100.0 \\
\hline
\end{tabular}




\section{REPORT DOCUMENTATION PAGE}

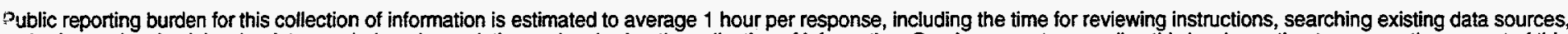

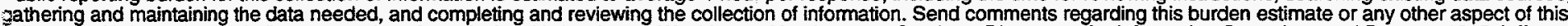

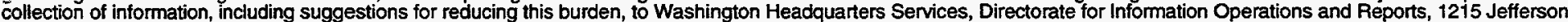

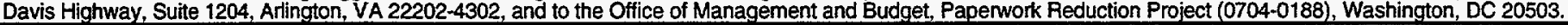
1. AGENCY USE ONLY (Leave blank)
2. REPORT DATE
3. REPORT TYPE AND DATES COVERED
September1997
Subcontract report

\section{TITLE AND SUBTITLE}

Determination of Alternative Fuels Combustion Products: Phase I Report

6. AUTHOR(S)

K.A. Whitney

\section{PERFORMING ORGANIZATION NAME(S) AND ADDRESS(ES)}

Southwest Research Institute

6220 Culebra Road

San Antonio, TX 78228

9. SPONSORING/MONITORING AGENCY NAME(S) AND ADDRESS(ES)

National Renewable Energy Laboratory

1617 Cole Boulevard

Golden, CO 80401-3393

8. PERFORMING ORGANIZATION REPORT NUMBER

(C)YAW-3-13253-01

(TA) FU703630

\section{FUNDING NUMBERS}

\section{SUPPLEMENTARY NOTES}

12a. DISTRIBUTION/AVAILABILITY STATEMENT

National Technical Information Service

U.S. Department of Commerce

5285 Port Royal Road

Springfield, VA 22161

\section{2b. DISTRIBUTION CODE}

UC-1504

13. ABSTRACT (Maximum 200 words) This report describes the laboratory effort to identify and quantify organic exhaust species generated from alternative fuel light-duty vehicles operating over the Federal Test Procedure on compressed natural gas, liquefied petroleum gas, methanol, ethanol, and reformulated gasoline. The exhaust species from these vehicles were identified and quantified for fuel/air equivalence ratios of $0.8,1.0$, and 1.2, nominally, and were analyzed with and without a vehicle catalyst in place to determine the influence of a catalytic converter on species formation.

\section{SUBJECT TERMS}

Alternative fuels, transportation fueis, air quality, combustion

15. NUMBER OF PAGES 150

16. PRICE CODE

\section{SECURITY CLASSIFICATION OF REPORT}

\section{SECURITY CLASSIFICATION OF THIS PAGE}

19. SECURITY CLASSIFICATION OF ABSTRACT
20. LIMITATION OF ABSTRACT

Standard Form 298 (Rev. 2-89) Prescribed by ANSI Std. Z39-18 